$$
Q L
$$

430.5

B8W52

1897

MOLL.

Westerlund

1897

Synopsis molluscorum extramarinorum regions

Palaearcticae 

Hesterlund, Caralo Agardh 1897

Synopsic mallusasum extramarinoun

-regionis Palalasclicie.

Division of Mollusiles

Gectional Library 



\title{
SYNOPSIS
}

\section{MOLLUSCORUM EXTRAMARINORUM}

\author{
REGIONIS PALÆARCTICE.
}

AUCTORE

Dr. CAROLO AGARDH WesterLUND.

\author{
Y FASCICULU' I. \\ GENERA ET SPECIES EX TYPIS \\ BULIM ET PUPE. *
}

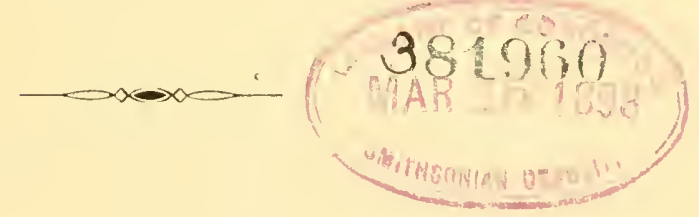

L UND 1997.

EX OFFICINA HAKKAN OHLSSON. 



\section{Synopsis}

\section{M oll us col'u m Extra ma ri in or u m Regionis Paladetica.}

\section{1. Malacozoa Cephalophora.}

Ordo 1. I | 0 | | e e I c $\mathbf{~} \mathbf{I}$ a $\mathbf{t}$ a.

Subordo 1. Geopliila.

\section{Sectio 1. M on otrema W.}

\section{Subsectio 2. Gnatophora.}

Fam. H e l i c i d s.

Subfam. 2. Pupina.

T. ovata, cylindracea, turrita vel elongato-fusiformis; apertura verticalis, longior quam latior, columella basi numquam truncata.

\section{A. Ex typo Bulimi.}

T. sæpius dextrorsa, ovato-conoidea ad turrita vel cylindracea; ap. ovato-oblonga, nunc et sæpius edentata, nunc dentifera, sæpiss. quam spira brevior, margine exteriore longiore.

\section{Conspectus generum.}

† A p. ed e nt at a (ad summum tuberculo angulari).

C y l i n d r us Fitz. T. cylindrica, perobtusa, pallida, striatula; anfr. 7-8, lati, plani; ap. ovalis marg. late disjunctis, valde inæqualibus, columellari cum columella continua. Austria merid. 
S e s t e r i a B. T. cylindriformis, fragilis, multispirata, col. cava, superne recta, iuferne percochleata, in arcu robusto ad medium aperturæ descendente. -- Mesopotamia.

B u I i u i u u s Ehrbg. T. (raro sinistrorsa) cylindrata, cornea v. albida (raro brunuea); anfr. 6-9; ap. ovalis, perist. sæpiss. in sp. Asiæ occ. late expanso, interdum reflexo, incrassato, in sp Asiæ centr. expansiusculo subsimplici, marg. conniventibus, callo særiss. juuctis, col. pliciformi. - Asia. (Petræus Alb.)

* N a | a u s Alb. T. (rariss. sinistrorsa) conico-turrita, corneo-brunnea (raro pallida); anfr. 6-9; ap. rotundato-ovalis, marg. subæqualibus, exter. ab insertione curvato, perist. sæpius recto, simplici. -

* I. e u c o c li i l o i d es Pfr. T. parva (1. 4-6 mm.), elongato-ovata, coruea; anfr. 5-7, ult. ascendens; ap. tuberculo angulari parvo, marg. approximatis, conjunctis, perist. late planeque dilatato. - Asia; Algeria.

* 0 il p h a l o c 0 ul u s W. T. late umbilicata, e basi ventroso forte conica, tenuis, coeruleo-albida; anfr. 5, teretes, perangusti; ap. fere circularis, marg. valde approximatis, perist. acuto, simplici. - Alg.

* Reto w s k i Bttg. T. pupæformis, ovata, subito conica, corneo-fusca, costulato-striata, dense granulata; anfr. $7-8$; ap. columella ad basin plica tortuosa, truncata, perist. late reflexo. - Caucasus.

* Il a d e a Bttg. T. ovati-conica, tenuis, alba v. carnea, (sæpe zona fusca mediana, tenuiss. spiraliter lineata; anfr. $5 \frac{1}{2}$ -8; ap. magna, marg. remotis, perist. toto expansiusculo. Caucasus, Asia.

* II a s | и s (Bk.) T. (rariss. sinistrorsa), elongatoovata, plus minus ventrosa (rariss. cylindraceo-turrita), flavidocornea ad olivacea; anfr. 6--9; ap. sæpiss. tuberculo angulari, marg. exteriore expanso, curvato, longiore. - Europa orient.merid., Alg., Asia occ.

* Z e b l i $\mathbf{l}$ us $\mathbf{s}$ Held. T. (raro sinistrorsa) ovato-conica ad cylindracea, alba (sæpe fusco-strigata); anfr. 7-11; ap. parva, marg. exter. recto, superue stricto, perist. labiato. Europa merid. (rariss. media) et Asia.

$$
+ \text { † } p \text {. lentata. }
$$

B re p h u l us (Bk.) T. (raro sinistrorsa), fusiformecylindracea $\nabla$. turrita (1. $10-26 \mathrm{~mm}$.), striata $\mathrm{v}$. striatula, 
alba (unicolor v. fuscostrigata), raro brunnea; anfr. 9-12; ap. angusla, marg. valde inæqualibus. - Eur. or.-merid., Asia.

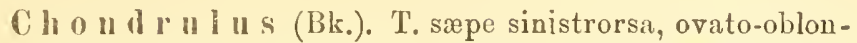
ga v. ovata, conica, acuminata, striatula, cornea; anfr. 6-9; ap. ovata, saltem in pariete et marg. exteriore crasse albolabiato dentata. - Eur. merid. (ruriss. media) et Asia.

* Se w e r tzo wi a Kob. T. breviter ovato conoidea, obtusa, costata (costis verticalibus, densis crassisque), alba; anfr. 6 , ult. valde ascendens; ap. callo parietali lamellam dentifornem intrantem exlibente. - Asia.

* A mp h is c o p us W. T. eylindraceo-turrita v. conico-fusiformis (1. $6-10 \mathrm{~mm}$.), pallida, lævigata; anfr. 7-11; ap. ovata, dentata, marg. subrqualibus. - Asia occ. et. Aegyptus.

\section{Gen. C y I i II d I u s Fitz. (Syst. Verz. 1833).}

C. obtusatus Drp. T. cæsio-albida, sæpius apice brunneo; ap. marg. colum. superne calloso, reflexo. L. 12, d. 3-4 mm. (Alpes Austrice). Hist. moll. 1805 c. f. Ic. f. 337. - Forma 1) conica W., t. sursum lente attenuata.

Gen. S e st er i a B. (Bull. mal. Fr. 1884).

S. gallandi B. T. tenuis; ap. obliqua, suboblonga, marg. callo forti conjunctis. L. 25, d. 5 , ap. 1. 6, d. 3 mm. (Mesopotamiz () . L. c. c. f. (B.)

Gen. B | I i u i n u s Ehrbg. (Symb. phys. 1831).

$$
\text { Conspectus. }
$$

† In Asia occidentuli.

1. T. cylindrica, crassa, apice rotundato-obtuso. (1.)

2. T. attcnuato-cylindrica, gracilior, accumimatre. $(2)^{\mathrm{s}}$.

ti In Asia centrali.

*) T. dextrorsa.

1. T. eylindrica, superne conoidea. (3.)

2. T. longe cylindraceo-turrita.

**) T. sinistrorsa. (5.) 
B. Iabrosus Oliv. T. cylindraceo-oblonga, lævigata, obsoletiss. spir.-lin., corneo-albida, apice breve obtuso; anfr. 7, planulati, superi regulares, ult. duo altiss., ult. totus postice lente attenuatus, sut. tenui, superne horisontali; ap. magna, ovalis, col. forte oblique plicata, perist. undique late expanso, nitido, callo par. tenui, extus sæpe tuberculifero. L. 27-33, d. 10-15, ap. 1. $16 \mathrm{~mm}$. (Syria, Palastina, Mesopotania). Voy. Ottom. 1807. Ic. f. 1324 .

Var. jordani Boiss. ap. Ch. (Zeitschr. 1847): t. ventricosior, anfr. convexiores, apice obtusiore, anfr. $51 / 2$, ult. altiore; J. 37 , d. $18 \mathrm{~mm}$. - Ic. f. $1322-23$.

Var. usplualtinus W. (B.-C. 1887): t. multo minor, cylindrica, apice obtu iore, coeruleo-albida, prope ap. forte densiss. spir. lin., anfr. 6. antepen. latiore, sut. medio obliquiore: 1. 21, d. 10, ap. 1. $9 \mathrm{~mm}$. (Mare mort.)

Var. diuninutus Mss. (Cog. Roth 1861): t. minor (1. 21-22. d. $10-12 \mathrm{~mm}$.), obtusior, nitida, ap. spiram fere æquante, paullo latiore. lc. f. 1326.

* B. granulatus W. Præc. aff., sed differt t. oblonga, sursum lentiss. attenuata, albocinerea, irregulariter leviterque striata, ubique densiss. tenue granulata, apice obtuso. L. 30, d. 12, ap. 1. $14 \mathrm{~mm}$. (Samaria). Verl. Ges. Wien 1892. - Forma 1) curta W. l. c., t. celerius ab ap. ad apicem attenuata, elongato-conica, l. 26, d. $13 \mathrm{~mm}$.

B. marsabensis $W$. $T$. cylindracea, apice rotundata, flavescens, nitidula; aufr. 6, regulares, sut. ut labrosus; ap. paullo truncato-ovalis, perist. ut labrosus, intus labio filif., callo par. contiuuo. L. $10 \frac{1}{2}$, d. $7 \mathrm{~mm}$. (Marsaba). Binn. Conch. 1886.

B. saboeanus B. T. cylindrica, superne subito acuminata, apice mamillata, cinereo-cornea, striata, supra medium lineis spiralibus pulcherrime granulata; anfr. 8, planiusculi, lente regulares, ult. non ascendens; ap. ovata, truncata, perist. ut labrosus, marg. colum. dilatato, callo par. tenuiss. L. 23, d. 11, ap. 1. $10 \mathrm{~mm}$. (Syria, Cypria). Sp. noviss. 1876. Loc. orig. Arabia ad Mareb (1. 22-23, d. $9 \mathrm{~mm}$. ; testa ex Cypria (Ic. Suppl. 1897) l. 21, d. 9 , ap. $1.8 \mathrm{~mm}$.

B. spirectinus B. T. elongato-cylindrica, apice obtusoattenuata, infra ad dextrum convexo-dilatata, pallide carneo-cornea, striatula, tenuiss. spir. lin.; anfr. 8, irregulares, ult. amplus; ap. oblonga, marg. exter. rotundato, reflexo, callo par. plus minusve valido. L. $36-38$, d. $11-12 \mathrm{~mm}$. (Syria). Sp. noviss. 1875. (B.) 
Var. Ihammastus B. l. c. : t. superne convexo-obesa, pallide cornea, tenuiss. spir. lin., anfr. 7, irregulares, ap. callo parietali tenni; b. 32 , d. $10-\mathbf{1 1} \mathrm{mm}$.

B. lamprostatus B. T. elongato-subcylindrica, superne obtuse attenuata, pallide corneo-carnea, ontidiss., eburneo-polita, læviss.; anfr. 7, sat celeriter crescentes, ult. convexiusculus; ap. oblonga, perist. eburneo, incrassato, validiss., reflexo, callo pariet. crasso. L. $22-23$, d. $8 \mathrm{~mm}$. (Libanon). L. c. 1876. (B.)

B. exaxastoma B. T. præc. similis, sed cornea, anfr. $7 \%$, regulares, ult. convexus, ad dextrum valde provectus, ap. ad dextram valde provecta, marg. colum. arcuato; 1.22 d. 7 min. (Libanon). L. c. 1876 . (B.)

B. therinus B. T. cylindrica, cornea, striatula, sub lente valido minutiss. tuberculis prædita, apice convexo-acuminata; anfr. $6^{1}{ }_{2}$, sat regulares, ult. valde ascendens, convexus; ap. oblonga, infra paullo angustata, perist. vix reflexiusculo, marg. colum. recto, callo par. valido. L. 17 , d. $6 \mathrm{~mm}$. (Tibanon). L. c. 1876 . (B.)

R. exochus B. T. ventroso-ovoidea, sp. ventroso-oblonga, obtusiss., pallide cornea, subopaca, striatula; anfr. $61 / 2$, sat velociter crescentes, ult. convexiusculus; ap. ampla, suboblongorotundata, callo par. tenui. L. 34, d. $13 \mathrm{~mm}$. (Tibanon). L. c. 1876 . (B.)

B. conrtieri B. T. elongata, cylindrica, cretacea, superne acuminata, cornea, apice aterrimo, striatula; anfr. 9, planulati, lente regulares, ult. basi subangulatus; ap. subcircularis, marg. valde approximatis, callo junctis. L. 20, d. $6 \mathrm{~mm}$. (Syria). L. c. 1876 . (B.)

B. fonrousi B. T. elongata, cylindrico-acuminata, sublævigata, passim malleata, violaceo-cornea, superne luteola; anfr. 9, lente regulares, planulati, ult. exiguus, vix convexus; ap. semioblonga, perist. leviter expanso, callo par. tenuiss. L. 15, d. $5 \mathrm{~mm}$, (Libanon). L. c. 1876 . (B.)

Var. dispistus B. 1. c. : t. fusif.-oblonga, snrsum attenuata, apice obtusa, anfr. 8 , sut. albidofilosa, ap. subrotundatooblonga, perist. plane expanso, marg. valde approximatis, callo tenui; l. 13 , d. $3 \mathrm{~mm}$.

B. neortus W. T. oblongo-subturrita, leviter obtusata, pellucido-cornea, nitens, striatula; anfr. 9, lente regulares; ap. ovata, ad marg. colum. verticalem angulata, perist. alboincrassato, dilatato, marg. disjunctis at tuberculo albo angulari. L. $20-22$, d. 7, ap. l. vix $8 \mathrm{~mm}$. (Mare mortuum). Binn.-Conch. 1887. Ic. f. 561 . 
B. syriacus Pfr. T. attenuato-cylindracea, albido-coerulescens, superne substrigata, apice colorato, sp. supra medium conica, striatula; anfr. $8-9$, regulares, planulati, infra angulati, ult. ascendens, basi obtuse angulatus, infra subtransversus; ap. truncato-ovalis, intus fuscula, marg. subæqualibus, leviter et xque curvatis, callo par. nullo at tuberculo parvo. I. 16 -20, d. 6-7, ap. 1. $7 \mathrm{~mm}$. (Syria). Symb. 1846 . Ic. f. 914.

B. mixtus W. T. oblongo-cylindracea, sp. oblongo-conoidea, supra medium corneo-brunıea, infra violaceo-lactescens, obsolete striatula; anfr. 8, infra subangulati, ult. lente ascendens; ap. oblonga, intus fuscula, marg. subæqualiter leviter curvatis. L. $14-16$, d. $4 \frac{1}{2}-51 / 2$ ap. 1. $5 \mathrm{~mm}$. (Antiochia acl. Zataka). Binn., Conch. 1887.

Ver, composiuns W. l. c. : t. turrita, striatula, sut. superne profunda et horizontalis, medio tenuis et obliqua, ad ap. ascendens, perist. anguste expansum; 1. 16, d. $5 \mathrm{~mm}$. (Sigoli.)

B. sidoniensis Ch. T. attenuato-cyliudracea, superne conica, sublævigata, cinereo-carnea v. rufobrunnea, nitida; anfr. S-9, superi convexi, sutura profuuda, inferi convexiusculi, nlt. demum subascendens, basi saccato-turgiclus; ap. truncato-ovalis, intus concolor; marg. exter. superne forte curvato, colum. subrecte verticali. col. intus late profundeque plicata, cum pariete angulum subrectum formante. L. $15-17$, d. 5-6 mm. (Syria). Zeitschr. f. Mal. 18t7. Ic. f. $91 \%$

I'ar. Emajor Kob. Ic. Suppl. 1897 c. f. : t. elongata, conico turrita, superne celeriter attennata; 1. 2J, d. $81 / 2-9$, ap. 1.6 mm. (Celicia.

B. niveus (Parr.) Rm. T. attenuato-cylindracea. alba, striatula, apice conico. obtusato; anfr. S. ult. paullo ascendens. striatus, basi compressiusculus; ap. subrotunda, perist. patulo, marg. subæqualibus, exter. magis curvato, tuberculo par. parvo. L. 13, d. $5 \mathrm{~mm}$. (Georgia, Tauria). Ic. f. 916. Rm.)

B. liotschyi Pfr. T. subcylindracea, sublævigata, fulvocarnea, nitida. apice ol,tuse conico; anfr. $9 \frac{1}{2}$, ult. subascendens; ap. ovalis, marg. convergentibus, exter. superne arcuato, colum. subdilatato. L. 24. d. $7 \mathrm{~mm}$. (Asia minor). Mal. B1. 1854. Ic. f. 1349 . (Pfr.)

Var. provectus n. : t. multo gracilior et minor, superne longius attenuata, anfr. uit. longe lenteque attenuatus, ap. oblonga, ad ilextram provecta; l. 15, d. $5(16: 41 / 2) \mathrm{mm}$. (Urfa.) Ic. f. 1350 (f. minor).

B nogelli Roth. T, subcylindracea. supra medium conico-attenuata, striatula, fulvo-cornea; anfr. 9, lente regulares, ult. basi forte attenuatus; ap. sexuiovalis, marg. distantibus, 
inæqualibus, exter. leviter curvato, colım. dilatato, plano, callo par. nullo. L. $17-19$. d. $5^{1 / 2}$, ap. 1. $5_{2}^{1 / 2} \mathrm{~mm}$. (Asia minor, Persia ad Tokat). Wagn. Reise 1855.

Far. juactas n. : t. minor , $1.16 \mathrm{~mm}$.), ap. marg. callo extus tuberculifero junctis. (Libanon.) Ic. f. 1959.

$B$ carneus (Pfr.) Phil. T. cylindracea, apice obtuse conica, stritula, nitida. sordile carnea; anfl. 9, plani, lente crescentes, ult. paullo ascendens, sut, marginata; ap. lata, semiovalis, perist. nitido. marg. exter. expanso, colum. dilatato, patente. I. 26 , d. 9 mm. (T.ycia, Palcestina, Cypria). Abbild. c. f. Ic. f. 1986 .

Var. glabaratura Mss. Coq. Roth 1861: t. minor, gracilior, tenuis, fragilis, glaberrima, lacteo translucens, ap. marg. filoso reflexo, non interrupto, al insertionem colum. sensim incurvato; 1. 17-20, d. $5 \frac{1}{2}-7 \mathrm{~mm}$. -- Ic. f. 1987. (Mss.)

B. eremita (Bens.) Reeve. 'T. periomphalo lato, obese subcylindrica, a medio convexo-conica, irregul. striata, cretaceoalba, raro griseo-flammulata; anfr. 7, sat celeriter crescentes, penult. nulto latior, ult. lente declivus; ap. parum oblique truncata, intus hepatica, perist. sublabiato. L. $18-22$, d. $8^{1 / 2}$ ... 10, ap. 1. $7-71 / 2 \mathrm{~mm}$. (Asia centr.). Icon. - Ic. f. 578. - Forma 1) minor, 1. 15-17, d. 7-8 mm. 2) cornere unicolor.

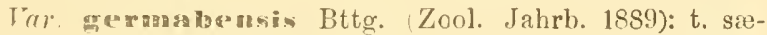
pius multo major, rentricosior, magis ovata, acutior, unicolor v. strigata, anfr. penult, convexior, ult. altior, ap major, intus flarescens; 1. 19-2R, d. $10-14$, ap. 1. 8-12/2 mm. (Trans. caspia.)

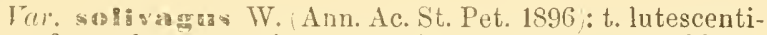
albida, anfr. multo convexiores, sut. impressa, superne sublorizoutalis, infra longe forteque ascendens; 1. 18, d. $8 \mathrm{~mm}$. ('Transcaspia).

I3. Iabiellus Mts. T. periomphalo angusto, obeso-cylindrico-ovata, pupæf,, læviss. striatula, carneo-albida, sparsim cormeo-strigata; anfr. $71 / 2-8$, regulares, ult. ascendens, basi sat leute declivis; ap. perist. valde incrassato, albo, marg. exter. superne recto, colum. obliquo, subcalloso. L. 14, d. 5-6, ap. 1. 6 mm. (Dschungaria). Conch. Mitth. 1980 c. f. Ic. f. 581. - Forma 1) minor, 1. 10-12, d. $4 \mathrm{~mm}$; 2) cornert, unicolor.

Var. strophostoma Anc. (Bull. soc. Fr. 1886): t. densius strigata, anfr. ult. altius ascendens, ap. non verticalis.

B. bonvallotianns Anc. T. oblongo-turrita, pallidiss. coffeo-lactescens, strigis pallide brumneo corneis numerosis, sp. regul. cornico-attenuata; anfr. 7-9, modice regulares, ult. basi 
subsaccatus, subtransversus; ap. perist. angustius expanso, marg. colum. dilatato, reflexo. L. 18-19, d. $7-8 \mathrm{~mm}$. (Turkestan). L. c. 1886 . Ic. f. 570, 572. Forma 1) minor (Ic. f. 571), 1. 15, d. $6^{5} / 2 \mathrm{~mm}$.

Vai. sumarlomulensis Kob. (Ic. f. 368-69): t. cylindracea, tantum apice panllo elongato-conica; 1. 20-24, d. $8 \mathrm{~mm}$.

B. ufjaloyamus Anc. T. longa, cylindrico-attenuata, apice conoidea, grisescens, strigis numerosis corneo-fulvis picta; anfr. ad $91 / 2$, lenti, medii subæquales, ult. postice lente declivis; ap. parva, ovalis, perist. modice expanso, leviter incrassato, marg. exter. sat curvato. L. ad 24, d. $71 / 2$, ap. $1.6 \%$ m. (Turkestan). L c. 1886. Ic. f. 576-77.

13. alaicus Kob. Præeced. siwilis, sed t. elongato-cylindrica, sensim leniter attenuata, pro relatione gracilis, obsolete strigata; anfr. 9-10, perlente (inferi-4-5 vix) crescentes, ult. postice vix $3 / 7$ long. testæ, ad ap. planatus et subimpressus; ap. ovalis, parva. L. 20, d. 6 , ap. $1.6 \mathrm{~mm}$. (Turkestan, m. Alai). Ic. f. 579 . (K.)

13. kokhandensis Mts. T. subcylindrica, in summo conica, coeruleo-alba, fuscostrigata, spir. lin.; anfr. $7-8$, lenti, 2 præult. subæquales, ult. postice longe lenteque attenuatus, infra breve transversus; ap. intus cornea. marg. exter. superne recto, deinde breviter expanso. L. $17-18$, d. $61 / 2-7$, ap. $7 \mathrm{~mm}$. (Turkestan). Centr. as. Moll. 1880 c. f. Ic. f. 586. - Forma 1) minor, $1.14^{2} / 2-15$, d. $4^{1} / 2-6$, ap. $1.5 \mathrm{~mm}$.

B. kuschakewitzi Anc. T. elongata, cylindraceo-attenuata, lacteo-alba, fusco strigata; anfr. 8. sat lente regulares, ult. usque ad ap. lente attenuitus, superne lente ascendens; ap. perist. expanso, dilatato, incrassato. L. 17. d. $51 / 2$, ap. I. $5 \mathrm{~mm}$. (Turkestan). L. c. 1886 . Ic. f. 585. - Forma 1) cundisata Anc., t. obsolete, distanter et tenue strigata, flavescenti-albida, palato fusco.

B albiplicatus Mts. T. cylindrico-oblonga, alba, ubique plicato-striata, interstitiis corneis; anfr. $71 / 2$, lente regulares, 2 præult. subæquales, ult. basi rotundatus; ap. marg. exter. vix incrassato, ab insertione curvato, recto, colum. dilatato, patente. L. 12, d. 5, 1. ap. $4 \mathrm{~mm}$. (Turkestan). Moll. Turkest. 1874 c. f. Ic. f. 583.

Var. maryucnsis Mts. ap. WV. (Ann. Ac. St. Pet, 1896): t. oblongior, lavigata, ap. oblonga, marg. subæqualibus, wqualiter arcuatis et expansis, tuberculo minimo angulari.

B. trigonochilus Anc. T. cylindracen, sublævis, fulvocornea, apice breviter conico; aufr. 9, lentiss. regul. crescentes, sut. vix obliqua; ap. sat distorta, subtrigona, marg. colum. 


\section{Abbreviationes.}

T. vel $t .=$ testa.

C. vel c. $=$ concha.

anf $r$. = anfractus.

a. pen. = antepenultimus.

penult $=$ penultimus.

ult. $=$ ultimus.

2 (duo) proult. = anfr. penult. el ult.

regul. $=$ regulariter.

irregul. = irregulariter (anfr. superi lente, inferi celeritex crescentes).

sc. spir. = sculptura spiralis.

sp. lin. = spiraliter lineata.

sut. = sutura.

nitidiss, profundiss. \&c. - nitidissima, profundissima \&c.

jusif., cylindrif., puntif. \&c. = fusiformis, cylindriformis \&c.

und. $=$ umbilicus.

umbil. = umbilicata.

perf. = perforata.

rim.-perf. = rimato-perforata.

sp. = spira.

atten. = attenuatus.

ap. = apertura.

marg. = margo.

exter. = exterior.

inter. $=$ interior.

perist. $=$ peristoma.

col. = columella.

colum. = columellaris.

par. $=$ parietalis.

$2-2-4$ vel $1-1-1 \& c_{0}=$ numeri dentium, lamellarum vel plicarum in apertura in hoc ordine: parietales, columellares et marginales (palatales).

1. 2. 3. 4. \&c. = lamellæ, plica et dentes in apertura a superiore (exteriore) ad inferiorem numerati.

pr. $=$ prope.

Abbreviationes ceteræ usu receptæ per se compreheuda sint.

Obs. Abbreviationes omnes allatæ per casus omnes ut convenit flexuose sunt. 

obliquo, dilatato, expanso, exter, verticali, recto, brunneolabiato. Long. 12, d. $4 \frac{1}{2} \mathrm{~mm}$. (Turkestan). L. c. 1886. - Forma 1) minor, l. 10, d. $4 \frac{1}{2}$ mm.. anfr. 8. (Anc.)

Var. teres IV. l. c. : t. omnino cylindrica, apice in conum breviss. contracto, fusca, anfr. 11, sut. obliqua; l. 12, d. $3^{1 / 2} \mathrm{~mm}$.

Var. expansilabris W. l. c. : t. cylindracea, apice breve conicn, rufobrunnea, ad ap. pallida, aufr. 9-10, sut. medio subhorizontali, infra valde descendente, ap. vix distorta, ovato trigona, perist. expanso, albo, crasso, nitido, callo apert. tuberculifero; l. 11 , d. $3 \frac{1}{2} \mathrm{~mm}$.

Íar. planicollis W. l. c. : t. cylindrica, fusco-brumnea, anfr. 9, ult. postice longe planeque attenuatus, sut. medio perobliqua, infra subhorizontali, ap. parum distorta, oblongoovalis, albolabiata; 1.11 , d. $3{ }^{\prime}{ }_{2} \mathrm{~mm}$.

I'ar. parluychila W. l. c. : t. olesa, crassa brevisque, forma 0 . dolii, fusco-cornea, apice breviss. conico, anfr. $8-8^{1 / 2}$, ult. postice convexus, basi subsaccatus, sut. fusco-marginata, paullo obliqua, valde ad ap. ascendens, ap. marg. crassis; l. 8 $-\mathrm{S}^{1} / 2$, d. $31 / 2-4 \mathrm{~mm}$.

B. ferghamensis Kob. T. elongata, cylindrica, apice conico, obtusato, leviss. striatula, cinereo-albida, strigis castaneis vividis, numerosis; anfr. 8, lentiss. crescentes, ult. penultimo angustior; ap. vix excisa, perist. continuo, albo, incrassato, fere undique reflexo. L. 13 , d. 4 , ap. $1.4 \mathrm{~mm}$. (Turania). Ic. f. 588.

B. warentzowi Rosen. T. parva, perf., cylindrica, gracilis, cornea strigis tenuibus albidis, nitida, apice obtuso; anfr. 7, lentiss. crescentes, superi convexi, sut. medio obliqua, albomarginata, ult. convexus, basi rotundatus; ap. parva, infra recedeus, marg. approximatis, exter. recto, tenui, ab insertione curvato, colum. verticali, dilatato, reflexo. "L. $7^{1 / 2}, \mathrm{~d} .2^{3} / \mathrm{s}$, ap. l. $2^{1 / 4} \mathrm{~mm} . "$ (Transcaspia, Gaudau). Congr. Int. Mosc. 1892. -Forma 1) minor, t. cylindraceo-oblonga, anfr. $6^{1} / 2,1.6 \frac{1}{2}, \mathrm{~d} .2 \mathrm{~mm}$; 2) major, cylindrica, anfr'. 9, 1. 9, d. $2 \mathrm{~mm}$.

B. purus W. T. longa, cylindrica, superne conica. lævis, alba, unicolor; anfr. 9--10, supremi lente crescentes, convexiusculi, tres inferi lati, planulati, ult. antice et postice longe lenteque attenuatus, perist. callo conjuncto. L. 17, d. $3^{1} / 2$, ap. l. $4 \mathrm{~mm}$. (Persia). Binn.-Conch. Suppl. 1890

Var. improbus W, 1. : t. brunnescens, superne fortius conica, anfr. penult. distincte altior, callus pariet. validus, perist. crassius, marg. colum. magis curvatus; $1.15, \mathrm{~d} .3^{2} / 4 \mathrm{~mm}$.

B. albocostatus W. T. periomphalo dilatato, cylindracea, superne longe attenuato-conica, pallide brunnea, forte et sat distanter costata (costæ albæ, verticales); anfr. 10, lentiss. crescentes, ult. parum major; ap. parva (1/6 long. testæ), marg. 
callo junctis, exter. recto, colum, dilatato, reflexo. L. 12, d. $31 / 2 \mathrm{~mm}$. (7'urkestan). Ann. Ac. St. Pet. 1896.

lar. aistortus W. 1. c. : anfr. ult. penultimo postice (3uplo, antice triplo altior, ap. forte ad dextrum listorta, marg. callo perobliquo junctis.

IP. pullaster (Mts.) Anc, T. cylindrica, apice conico, subcornleo-albida: anfr. $7 / 2$, convexi. lente regulares, sut, profunda. ult. superne rectus, basi rotundatus; ap. parva, infra rotumelata. intus alba, perist. tenui, subsimplici, vix callo juncto, marg. colum. superne dilato. L. 10, d. 4, ap. 1. 31 2 mm. (Turkestan). L. c. 1826 . Ic. f. 590.

13. erpans W. T, oblongo-cyliudracea, superne conicoattenuata, corneo-bruvuea, costata (costæ sat clensæ, obliqux, albr, obtuse, recta); anfr. $7 \%$, regulares, ult. ascendens, basi rotuudatus: ap. ovalis, col. superue plicif. callosa. perist. toto late reflexo, incrassatulo, fusculo. L. $12-13$, d. $3 \frac{1}{2} \mathrm{~mm}$. (Turkestan). Amu. Ac. St. Pet. 1896.

3. costatus Cl. T. cylindracea, apice breve conico. rufescenti-brunnea, albocostata, costis ap. versus densioribus et temuioribus; anfr. 7 , conrexi, lentiss, regulares, ult, paullo ascendens, bisi fere angulatus; ap. ovata, perist. acuto, albo, patulo. I. (i. d. $3^{i}{ }_{5} \mathrm{~mm}$. (Trorliestan). Nichr. bl. 18:4. Cl.)

B. castaneus n. T. subcylindrica, apice breviter conico, lavigata, vitida, fere castaneo-brumea, ad ap. late flavescens; anfr. S, leutiss, regulares, sut. infra medium albofilosa, ult. lente alteque ascendens, infia leviter ac breve aftenuatus; ap. subtrigonalis, ad basin medio angulata, perist. intus rufolabiato, pistulo, iucrassato. callo par. medio sæpiss. abbreviato, ad marg. incrassato. L. 11, d. 4 mm, (Samarkanul). Sub nomine B. asiatici accepri.

B. leptoceras W. T, couico-turrita, temuis, lutescenticolnea, uitida, passim obsolete albido-strigata, leviter striatula, obsoletiss. dense spir. lin., sp. elougato-conica, teune attenuata; anfr. 9, supresui lenti, convexi, cateri sit lati, planiusculi, ult. deorsum lente attenuatus, sut. infra subdescendens; ap. ovatooblonga, intus fulvida, marg. versus late albida, perist. tenue, murg. exter. ab inscrtione leviter curvito, colum. superne dilatiato et reflexo, callo par. teuui. I. lis, d. \pm mm. (Persiu ad Siaret). Anm. Ac. St. Pet. 1996.

B. rimatus Pfr. T. oblougo-turrita, sat temuis, striatula, pallirle cormea; aufr. 7. subplani; al oblongo-ovalis, perist. simplici, murg. approximatis, conjunctis, exter, vix expausiu- 
sculo, colu'u, dilatato. L. 33, d. 11, ap. 1. 13 mm. (Afghtanistem). Proc. Lond. 1846. I'fr.!

13. longulus Rolle. T. aperte perf, elongato-turrita, sp. primum cylindrica, deinde conica, rufogrisea. olsolete saturittius strigata, striatula; anfr. $11-12$, superi convexi, ap. ovatoacuminatit, basi patula, callo supra marg. colum, plicif. prominulo, perist. breviss. reflexo, subremote labiato. L. 30, d. 7, ap. 1. 9 num, (Palrestina). Naclur. bl. 1993. Ic. f. 1199. R.

B. davidi Desh. T. turrita, subfusif, irregul, striata, castanea, strigis angustis cretaceis; anfr. 10, ultimi 3 celerrime crescentes, plannlati, ult. convexus, antrorsum angustatus; ap. elongato-ovata. intus violaceo-castanea, marg. exter. tenui, expanso, colum, valde dilatato. L. 29. d. $8 \mathrm{~mm}$. (Thibet). Moll. Thib. $187(1).(1 \%)$

B. grillithi (B-ns.) Pfr. T. subfusif, elongata, gracilis. sp. subturrita, cretacea. rugosa; anfr. 8, planulati, ult. basi attenuatus, subcompressus; ap. magna, ovalis, intus caruea, perist. sulblabiato. marg. exter. dilafato. colum. substricto, dilatato, patulo. I. 28, 1. 8, ap. 1. 11 mm. (Afghanistun). listr. in Chemm. Cab. c. f. Pfr.

13. sindicus (Bens.) Reeve. T. cylindraceo-turrita, striatula, griseo-alba, strigis paucis pallide corneis angustis, sp. turrito-oblongal; anfi. $S^{\prime / 2}$, subplanati, ult. basi paullo compressus; ap. intus allo, albolabiata, marg. subæqualibus, exter. expansiusculo, colum. dilatato, patulo. L. 21, d. 7, ap. 1. 61/2 mm. (Hindostan, Afghanistan). Ic. Pfr.

IB. bamloni Desh. T. elougato-turrita, paullo fusif, apice acuto, irregul. plicato-striat, cretacca, rufocorneo-strigata; anfr. 9 , lente crescentes, convexi, sut. profunla; ap. ovata, intus albia, perist. incrassato, reflexo, sulscontinuo, marg. colum. valde dilatato. L. 16, d. $5 \mathrm{~mm}$. (Thibet). L. c. 1871). (D.)

B. moupinianus Desh. Precerl. similis, sed. t. ventriculosi, fuscol,runnea, leviter allostrigata v, maculata. obsolete striata; anfr. 8, ultimi celeriter crescentes; ap. perist. tenui, anguste expanso, marg, colum. angusto. L. 14, d. 5 mm. (Thibet). I. c. 1870. D.

B. trojanus Kob. T. eloagata-sul,fusif, leviuscula, lutescenti-coruea, sp. apice conica; anfr, 10-11, inferi fere plani, 2 ultini angustati, ult. basi compressus, pone ap. planatus; ap. ovata, ad dextrum producta, marg. exter. albolabiato, medio incrassato, vix brevisz. expanso, callo pariet. fere nullo. L. 14, d. $3{ }_{4}^{3 !} \mathrm{mm}$. (Troas). Ic. f. $110 \mathrm{n}$. Kol.

13. segregatua (Bens.) Iincve. T. perf, paullo turritooblonga, tenuis, striatula, flivido-brunnea; anfr. 7, ult. basi ro- 
tundatus; ap. marg. distantibus, exter. tenui, simplici, subrecto, colum. a basi dilatato, patulo. I. 13, d. 7 , ap. $1.5 \mathrm{~mm}$. (Himalaya occ.). L. c. (Pfr.)

B. asterabadensis Kob. T. cylindraceo-fusif, alba, fuscostrigata, sp. turrita, sensim attenuata; anfr. 91/2, lente crescentes, sut. impressa; ap. ovata, marg. exter. reflexiusculo, valde incrassato, col. obsolete plicata, callo pariet. utrinque subincrassato. L. 16, d. $4^{1 / 1}$, ap. $1.4^{1 / 2} \mathrm{~mm}$. (Persia). Ic. f. 2039. (Kob.)

B. subtilis Rm. T. cylindrica, apice attenuato, substriata, cornea, cervice tumido albocincta; anfr. 9, sut. tenui, albida; ap. acute ovata, marg. exter. albolabiato. L. 14, d. $4 \frac{1}{2}$, ap. 1. $4 \mathrm{~mm}$. (Dalın., Rumelia). Ic. f. 392.

Var. corcyrensis Mss. (Coq. Schl. 1859): t. minor, gracilior, pallidior, anfr. 9-10;1. $9 \mathrm{~mm}$. (Corfu.)

B. subulatus $\mathrm{Rm}$. T. cylindrica, longe lenteque attenuata, sublævis, cinerea, brunneo-strigata; anfr. (8: Rm.) 10II, sut. impressa, superi convexi; ap. acute semiovata, perist. subsimplici, recto. L. $10-11$ (14), d. $21 / 2-3$, ap. $1.2_{1 / 2}^{1 /} \mathrm{mm}$. ('Tauria). Ic. f. 393.

B. goldfussi Kob. T. cylindrica, apice breve conico, rufobrunnea; anfr. $8^{1 / 2}$, lentiss. crescentes, sut. albomarginata, fere plani, ult. basi compressus, antice planatus; ap. fortiter lutcolabiata, callo pariet. tenui, marg. exter. recto, colum. multo breviore, superne reflexo. L. 10, d. $4 \mathrm{~mm}$. (Turkestan). Ic. f. 1102 .

B. kunawurensis (Hutt.) Recne. S. subcylindrica, lævigata, sub sut. rugosula, cormea, albidostrigata, sp. sursum lente attenuata, acuminata; anfr. 12, lentiss. crescentes, ult. basi rotundatus; ap. intus albida, labiata, marg. conniventibus, exter. expanso, curvato, colum. multo breviore, dilatato. L. 32-35, d. $71 / 2$, ap. $1.8 \mathrm{~mm}$. (Thilet). L. c. (Pfr.)

B. candelaris Pfr. T. cylindracea, sursum lente attenuata, acuminata, sordide albida; anfr. 9, planulati, regulares, ult. basi rotundatus; ap. intus alba, nitida, perist, undique breviter expanso, albo, marg. distantibus, tenue conjunctis, colum. dilatato. L. 27, d. 8, ap. 1. $81 / 2 \mathrm{~mm}$. (Persia, Thibet). Non. 1848. (Pfr.)

B. desgodinsi Anc. T. aperte et profundiss. umbilicata, breviter cylindrica, ventrosa, superne convexa ac subito attenuata, apice valido, subcyaneo-lacticina, sursum magis magisque 
cornea; anfr. 7, medii subplanati, ult. antice convexus, rectus, infra angustatus; ap. intus fuscocastanea, perist. candido, breviter patulo, marg. approximatis, callo pariet. valido. I. 25, d. 10, ap. 1. $10 \mathrm{~mm}$. (Thibet). Bull. soc. Fr. 1884. (Anc.)

Var. codopsis Anc. l. c. : t. minor, ventrosior, umbilicus latior, anfr. medii sat convexi, ult. antice ascendens; ]. 20 d. 10, ap. 1. $10 \mathrm{~mm}$.

B. dominus (Bens.). T. rimato-perf., ovato-cylindracea, striatula, albida, rufobrunneo-strigata, sp. sursum celeriter attenuata, acuminata; anfr. 8 , ult. basi rotundatus; perist. undique dilatatum, callo pariet. brevi, marg. exter. superne curvato. L. 23, d. $9 \mathrm{~mm}$. (Kaschmir.). Ann. a. Mag. 1857. (Bens.)

B. perrieri Anc. T. rimata, cylindrica, apice valido prorsus in conulum attenuato, striatula, cornea, cum zonula albida ad sut. (post mortem uniformiter candida); anfr. 8, plauiusculi, lente regulares, ult, convexus: ap. intus cornea, marg. callo valido crasso junctis, exter. rotundato, colum. stricto. L. 18, d. $6^{1} / 2$ ap. 1. $6 \mathrm{~mm}$. (Thibet). Bull. soc. Fr. 1884. Forma 1) minor, l. $14-15$, d. $6 \mathrm{~mm}$. (Anc.)

B. boysianus (Bens.) Recve. T. subperf., turrito-oblonga, striata et tenue spir. lin., olivaceo-cornea, flavido-strigata, sp. lente attenuata; anfr. 8, subplani, ult. ventrosus; ap. perobliqua, perist. tenue labiato, marg. exter. recto, acuto, forte curvato, colum, verticali, superne reflexo. L. $171 / 2$, d. $61 / 2$, ap. 1. 5 mm. (Thibet). L. c. (Pfr.)

B. rochebruni Anc. T. perf.-rimata, ovoidca, curta, ventrosa, medio breviter cylindracea, apice obtuse conico, argute striatula, pallide cornea; anfr. 7, lentn regulares, medii subplanulati, ult. convexus, superne rectus; ap. extus convexa, ad col. recta, perist. candido, obtuso, marg. remotis, callo pariet. junctis. L. 17, d. 7, ap. 1. 51/2 mm. (Thibet). Bull. soc. Fr. 1884. (Anc.)

N a p a u s Alb. (Heliceen 1850).

Conspectus.

$\dagger T$. dextrorsa.

*) In Africa bor.

1. T. attenuato-conica, sape subturrita, pallida, sapius strigata; anfr. ult. basi rotundatus. (1.)

2. T. obtuse ovata, medio sape turgido-ventricosa, spir. lin., apice subito conico, anfr. ult. basi rotundatus. (2.)

3. T. infra ventricosa, sp. breve conica, anfr. ult. basin versus anyulato-carinatus. (3.)

**) In Europa (pracipue merid.-orient.) et Asia occ.

4. T. magna, ovato-conoidea $v$. cylindraceo ovata albida; ap. fere $1 / 2$ long. testre. 
5. T. conico-turrita, sp. conico attcnuata, striata, cornea $v$ fluvesecns. $\quad 5$.

6. T'. ovato-turita, cornea-olivacen, striata et spir. lin. 6.)

7. T. parva, elongato-ovata $v$. oblongo-conoidea, curneo-brunnca, striata. (7.)

***) In Asia centrati.

8. T. conica. (8.)

9. T. ovato-conoidea. 9.)

10. N. goebeti W. 10.)

$++T$. simistrorsa. (Tanria.) (11.)

N. kabyliauns Let. T. perf., elongato-ovata, coerulescenti-cinerea, indistincte brunneo-strigata, striatula, sp. sat regul. attenuato-conica; anfi. 7, sat celeriter crescentes, medii superne subdepressi, infra convexiores, ult, rotundatus, basi subsaccatus, sut. impressa; ap. oblonga, marg. subrectis, conniventibus, subæqualibus et æque curvatis, vix labiatis. L. $16-$ 18, d. 7, ap. 1. $6 \mathrm{~mm}$. (Ḱrbylia). Ann. mal. 1870 c. f. Ic. f. $618-620$.

rar. mansmeresis Kob. Ic. f. 624 : t. minor, umbilico latiore, anfr. planulatis, ap. marg. colum. angulum cum. marg. basali formante; 1. 14, d. 6, ap. 1. $6 \mathrm{~mm}$. (Algeria.)

N. milevianus laym. T. vix rimata, ovato-conica, cornea, unicolor, irregul. costulato-striata, sp. longe lentequeattenuata, apice magno; anfr. 7, lente regulares, sut. lineari, ult. major, basi attenuatus; ap. ovata, marg. exter. recto, tenui, arcuato, col. longa, recta, verticali. L. $14-17$, d. $7-8$, ap. 1 . 6-7 mm. (Algeria). J. Conch. 1853 c. f. Ic. f. 611 .

N. djurdjureusis (Anc.) W. T. rimato-perf., elongatoturrif., tenuis, cornea, lævis (ad ap. irregul. striata); anfr. $6^{1} 1 / 2$, superi regul., inferi celeriter crescentes, superi valde convexi, subcylindracei, sut. profunda, inferi convexiusculi, sut. lineari, ult. convexus; ap. elongato-ovata, marg. exter. recto, tenui, arcuato. L. $11^{1} / 2$, d. 4, ap. 1. $4 \mathrm{~mm}$. (Algeria). Verh. Ges. Wien 1892.

N. berthieri L. \& B. T. semiobtecte perf., elongatoconoidea, irregul. striata, cornea, sp. quam in mileviano graciliore; anfr. 7-8, valle convexi, lente crescentes, sut. profunda, ult. paullo inflatus, rotundatus; ap. perobliqua, ovata, basi recedens, marg. exter. tenui, acuto, col. brevi. L. $14^{1 / 2}$, d. 6, ap. 1. $5 \mathrm{~mm}$. (Algeria). Moll. Tun. 1887. Ic. f. 642 . (L. \& B.)

N. punicus L. \& B. T. fere obtecte rimata, elongatoconica, subfusif,, cornea, passim obsolete albidostrigata, striatula, 
sp. regul. attenuata; anfr. 7 , lente regulares, ult. inferne attenuatus; ap. perobliqua, augusta, elongato-suboblonga, marg. exter. recto, acuto, col. brevi. L. $15-16$, d. $5 \frac{1}{2}-7$, ap. 1. 5-6 mm. (Tunesia). L. c. 1887 . Ic. f. 643.

I. cirtanus Mor. T. rimata, oblongo-subfusif,, cornea, translucens, strigis et maculis albis opacis variegata, superne cornea, irregul, et vix striatula, sp. sensim conico-attenuata; anfr. 6 , celeriter crescentes, parum convexi, ult. basi rotundatus; ap. ovalis, intus alba, nitida, marg. exter. leviter expanso. L. 13 , d. $5 \mathrm{~mm}$. (Algeria). J. Conch. 1857. Ic. f. 2031.

N. todillus Mor. Præced. persinilis, sed t. vix rimata, elongata, subfusif., gracilior, solidior, irregul. striata, subcornea, forte albovariegata, anfr。 7, planiusculi, ult. basi plus minusve angulatus; ap. parva, elliptica, intus rufescens, labio lato, nitido, albo, perist. breviter expanso, L. 13, d. $4 \mathrm{~mm}$. (Algeria). L. c. 1858 c. f. Ic. f. 2032,63 .

Var. janus IV. (B.-C. 1887): t. late rimata, turrito. acuminata, strigis corneis albidisque variegata, anfr. 8 , omnes infra angulati, angulo prostante, superi convexi, inferi subplani; l. 15-16, d. $4^{1} / 2-5$, ap. 1. $5 \mathrm{~mm}$. - Ic. f. 638-639.

Var. cartensensis (Let.) Kob. (Ic. f. 637): t. tenuior, gracilior, strigis corneis peculiariter dispositis; l. 13/2, d. 6, ap. $1.5 \frac{1}{2} \mathrm{~mm}$.

N. letoumeuxi B. T. rimato-perf, ovato-conica, subturrita, irregul. striatula, subtiliss. spir. lin., cornea, unicolor; anfr. 7, lente crescentes, ult. major, inflatus, rotundatus; ap. intus concolor, ovato-rotundata, perist. teuui, patulo, reflexo, albolabiato. L. $12--13$, d. 6 , ap. 1. 5-6 mm. (Algeria). Mal. Alg. 1864 c. f. Ic. f. $634-635$.

N. chareius B. T. aperte perf., cylindrico-acuminata, griseo- v. albido-cornea. apice valido; anfl. 7, convexi, lente regulares, ult. convexo-rotundatus, ad dextrum valde excentricus; ap. suboblongo rotundata, ad dextrum valde provecta, perist. undique late reflexo ac expanso, marg. valde approximatis. L. 12 , d. $5 \mathrm{~mm}$. (Algeria). Sp. noviss. 1876 . (B.)

N. aristidis Anc. $T$. rimato-perf,, oblongo-attenuata, fusco- v, griseo-carnea, passim albido-maculosa, subtiliter striata, sp. conoidea; anfr. fere 7, convexi, ult. oblongus; ap. oblonga, extus convexa, perist. subsimplici, acuto, expansiusculo, marg. approximatis. L. 12, d. $4 \frac{2}{3}$, ap. 1. 4 mm. (Tunesia). Le Natur. 1888. (Anc.)

N. lecouffei Anc. T. aperte perf., elongato-conoidea, cornea, passim opace albomaculosa, apice submamillato; anfr. 
$6^{1} / 2$, convexi, regulares, ult. bene convexus, subsaccatus; ap. excentrica, subovalis, perist. acuto, expansiusculo, marg. valde approximatis. L. $11^{1 / 2}$, d. 5, ap. $1.4 \mathrm{~mm}$. (Tunesia). L. c. 1888. (Anc.)

N. jeannoti Terv. T. rimato-perf, ovato-conoidea, e basi ventrosula seusim sat bene attenuata, vix striatula, tenuiss. spir. lin., cornea, albovariegata; anfr. 6, regulares, ult, ventrosulus, basi leviter saccatus; ap. intus alba, ovato-rotundata, perist. expauso, tenue labiato. L. $11-12^{1 / 2}$, d. 6, ap. 1. 5 mm. (Algeria). Moll. Alg. 1864 c. f. Ic. f. 625 . - Forma 1) cornea B., minor, unicolor.

Var. blidalıensis Kob. (Ic. f. 626): t. gracilior, conica, solidior, anfr. ult. minor, marg. magis conniventes; l. 15, d. $61 / 2$, ap. $1.5 \frac{1}{2} \mathrm{~mm}$.

Var. olivetorum Koh. (Ic. f. 627): præced. sat similis, sed minus conica, potius fusif., perist. minus dilatato, labio fortiore.

$V a r$. thayacus Kob. (Ic. f. 628;: t. angustiss. perf., elongato conica, regul. attenuata, sordide alba, passim corneodiaphanomaculata, lin. spir. nullis, anfr. 7, medii superne planiusculi, supra sut. inflati et prominentes, ult. haud inflatus, ap. ovata, ad latus dextrum producta; l. 15, d. anfr. ult. 6, penult. 5, ap. 1. $4^{1 / 2} \mathrm{~mm}$. - Forma 1) zeugetana Kob. (Ic. f. 629), t. minor, conica, grisenalba, par um corneo-maculata, anfr. convexioribus et magis imbricatim supra sut. prominentibus; 2) lambasensis Kob. (Ic. f. 630), t. minus conica, paullo latius perf., maculis corneis numerosis sat obsoletis, anfr. regul. convexis.

N. bonensis W. T. rimata, oblongo-conica, irregul. striata, cornea, sp. convexo-conica; anfr. 7, regulares, ult. basi rotundatus, superue lente descendens, sut. albomarginata; ap. oblonga, marg. late distantibus, perist. patulescente, albolabiato, colum. dilatato. L. 12 , d. $5 \mathrm{~mm}$. (Algeria, Bona). Binn.Conch. 1887.

N. lhotellerii B. T. aperte perf., ventricosa, oblonga, ad sut. striatula, albidula, sp. acuminata, apice obtusissimo; anfr. 6, convexi, lente regulares, ult. convexus (1. $41 / 4 \mathrm{~mm}$.); ap. semioblonga, perist. incrassato, candido, ad basin leviter dilatato, marg. remotis, callo junctis. L. $91 / 2$, d. $4^{1 / 2} \mathrm{~mm}$. (Algeria). Sp. noviss. 1876. (B.)

N. guillaini Petit. T. vix rimata, ovato-elongata, densiss. regul. costulata (anfr. ult. antice lævior), cornea, strigis numerosis albis; anfr. 7, lenti; ap. parva, anguste ovata, intus alboincrassata, marg. distantibus, callo tenui junctis, marg. colum. plica obliqua. L. $14-15$, d. 6, ap. 1. vix $5 \mathrm{~mm}$. (Algeria). J. Conch. 1850 c. f. 
N. brondelianus B. T. rimato-perf, obtuse oblonga, ventricosi, fusco-olivacea, unicolor, striatula, sp. oblonga, obtusa; anfr. $71 / 2$, regulares; ap. semi-ovalis, marg. exter. recto, intus alboincrassato, colum. reflexo, stricto. L. 15, d. 61/2, ap. 1. $6 \mathrm{~mm}$. (Algeria, Calle). Nal. Alg. 18(t+ c. f. - Forma 1) elon. guta B., t. magis elongata, minus obtusa, $1.16, \mathrm{~d} .5^{1 / 2} \mathrm{~mm}$.

N. numidicus B. T. vix rimata, oblonga, sursum lente attenuata. striatula. cornea; anfr. 7, regulares, ult. rotundatus; ap. angulato-oblonga, valde excisa, marg. exter. allooincrassato, patulo, colum. extenso, reflexo, callo pariet. tenui. L. 15, d. 6, ap. $1.6 \mathrm{~mm}$. (Algeria). L. c. $186 \pm$ c. f. (B.)

N. vriesianus (Anc.) Kob. T. perf., ovato-oblonga, sordide lutescenti-alba, corneo-strigata. irregul. striata et malleata, sp. subito attenuata; anfr. $63^{1} / 2$. sat convexi, penult. gibbosus, super ult. prominens, ult. leviter attenuatus, pone ap. planulatus, basi rotundatus; ap. late ovata, alba, perist. acuto, incrassato, expanso, marg. colum. dilatato, reflexo. L. 12, d. $51 / 2$, ap. 1.5 mu. (Algeria). Ic. f. 631 . (Kob.)

N. bonrguignati Let. T. perf, obeso-ovata, medio turgido rentricosa, cœrulescenti-albida, vix striatula, distincte densiss. spir.-liu., apice subito breviss, conico, luteolo; anfr. 6, superi regulares, ultimi duo celeriter crescentes, penult. tumidus, ad sut, aigulato-prominens, ult. subinflatus; ap. oblonga, marg. exter. recto, acuto, arcuato, colum. verticali, subreflexo. L. $14-16$, d. $71 / 2-81 / 2$, ap. 1. $6-61 / 2 \mathrm{~mm}$. (Algeria). Ann. mal. 1870 c. f. Ic. f. $2029,623$.

Var. transiens Kob. (Ic. f. 622 ): t. minor, minus ventricosa, subovoidea; 1.12 , d. 6 , ap. $1.5 \mathrm{~mm}$.

N. bogharieusis Kob. T. rimato-perf, ovato-conoidea, infra sat ventrosa, sursum forte attenuata, ruditer striatula, subtiliss. spir.-lin., griseo-cornea; anfr. 6, convexi, regulares, ult. rotundatus, lente ascendens; ap. late ovato-rotundata, perist. subrecto, incrassato, marg. colum. vix dilatato. L. 10, d. 6 , ap. 1. $4^{1 / 2} \mathrm{~mm}$. (Algeria). Ic. f. 632 .

N. issericus Kob. T. rimato-perf, elongato-ovata, striatula, subtiliss. spir.-lin., albido lutescens, strigis flammisque ornata, sp. conico-turrita; anfr. 7, sat celeriter crescentes, ult. subcylindricus; ap. oblonga, lutescens, perist. tenui, fere undique reflexo. L. 15, d. 6, ap. 1. $6 \mathrm{~mm}$. (Algeria). Ic. f. 621 .

N. gaillyi (Let.?). T. anguste rimata, cylindrico-oblonga, supra medium conica, nitida, striatula, dense tenuiss. 
spir.-lin., rufocornea, anfr. ult. albido (t. mortua alba, superne lutea), costulato-striata; anfr. $7 \frac{1}{2}$, regulares, plauulati, ult. subattenuatus; ap. ovata, callo par. utrinque incrassato, marg. exter. leviter curvato, albolabiato, basali expanso. L. 16, d. 6, ap. 1. $5 \mathrm{~mm}$. (Aegyptus, Alexandria). Ic. f. 562-563.

N. micelii Kob. T. subobtecte perf., conoidea, striatula, cornea; anfr. $5-5^{1} !_{2}$, lenti; ap. ovata, obliqua, perist. subrecto, tenui, callo continuo, marg. basali dilatato. L. $9^{1} / 2$, d. 6 , ap. l. $4^{3} / 4 \mathrm{~mm}$. (Tunesia). Nachr. bl. 1885. Ic. f. 640 .

N. halepensis Pfr. T. cylindraceo-ovata, tenuis, nitida, corneo-albida, latere exteriore convexo, sp. convexo-conica, apice acuminato; anfr. 7, planiusculi; ap. rotundato-ovalis, fere dimidiam testæ attingens, callo par. nitido, marg. approximatis, nitidis, albis, exter. ab insertione leviss. curvato. L. 20, d. 8 (1. 17-23) mm. (Syria, Mesopotamia). Symb. 1841. Ic. f. 1327-29. (Pfr. \& Kob.)

N. mesopotamicus Mts. T. cylindracen-ovata, coerulescenti-alba, concolor $\nabla$. brunneo-strigata, opaca; anfr. 7, ult. basi paullo compressus; ap. elliptico-ovalis, intus ochracea, marg. approximatis, callo valido conjunctis. L. $17-18$, d. $7-81 / 2$, ap. 1. $7-7^{1 /} / 2 \mathrm{~mm}$. (Mesopotamia). Vorderas. Conch. 1873 c. f. (Mts.)

N. Iycicus Pfr. ap. Phil. T. rimato-perf., ovata, tenuis, lævigata, nitidiss., corneo-lutescens, sp. conoidea, apice obtuso; anfr. $61 / 2$; ap. ampla, semiovalis, callo par. tenui, marg. exter. intus albo, late expanso, colum. late reflexo. $L$. 25 , d. $12^{1 / 2}$, ap. $1.12^{1} / 2$ mm. (Iaycia). Abb. 11. c. f. Ic. f. 1985. (Pfr.)

Var. borenlis Mss. (Coq. Schl. 1863): t. minor (1. 20 $\mathrm{mm}$.), pallide cornea, translucida, paullo gracilior, col. subelongata, dentato-plicata. (Cancasus).

N. reitteri Mts. T. conico-turrita, leviter striatula, castanea, sp. sat regul. attenuata, apice globoso; anfr. 9, lentir ult. basi rotundatus, sensim attenuatus; ap. ovato-elliptica, callo par. diffuso, marg. exter. recto, superne forte arcuato, leviter incrassato, colum. subverticali, superne dilatato et reflexo-ap. presso. L. 14, d. $4 \frac{1}{2}$, ap. $1.41 / 2 \mathrm{~mm}$. (Montenegro, Dalmatia). Ber. Offenb. Ver. 1880. Ic. f. 567. (Mts. \& Kob.) 
N. rupestris Kryn. T. ovato-turrita, parum striata, flavescenti-cornea, sp. lente attenuata, apice truncatulo; anfr. 7 -8, lenti, ult. basin versus perconvexus; ap. ovalis, callo par. tenuissimo, marg. exter. reflexiusculo, præcipue superne valde arcuato, albosublabiato, colum. multo breviore, late dilatato. L. 17, d. 7 (20: 6) mm. (Tauria). Bull. Mosc. 1833 c. f. Ic. f. 385 .

N. merduenianus Kryn. T. perf., ab anfr. ultimo subgloboso ovato-turrita, corneo-lutescens, læviuscula, sp. sursum forte attenuata; anfr. 7-8, couvexi, regulares; ap. ovali-oblonga, callo par. diffuse incrassato, marg. exter. forte curvato, recto, simplici, colum. parum breviore, leviter dilatato. L. 13 15 , d. $5^{1} / 2-6^{1} / 2$ mm. (Tauria). L. c. 1833 c. f. Ic. f. 388.

N monticola Roth. T. elongata, oblongo-conica, corneofuscula, striatula; anfr. 8, planulati, regulares, sut. albida, ult. basi rotundatus, attenuatus; ap. ovalis, callo par. tenui, perist. albolabiato, late reflexo, marg. exter. superne valde curvato, coluın. verticali, leviss. dilatato. L. $15-21$, d. $6-7 \mathrm{~mm}$. (Groccia). Mal. Blätt. 1856 c. f. Ic. f. 1346.

N. Aryops Mts. T. perf., elongata, striatula, griseocornea, sp. turrito-cylindracea, gracilis; anfr. 9, regulares, ult. basi subangustatus; ap. rhombeo-oblonga, callo par. tenuiss., perist. expanso, albolabiato, marg. exter. superne curvato, colum. stricto, dilatato, introrsum subplicato. L. $14-18$, d. 4-5, ap. l. $5^{1} / 2 \mathrm{~mm}$. (Grecia). Moll. Oertz. 1889 c. f. Ic. f. 1108.

N. gracus Bk. T. ovato-oblonga, carneo-flavida, striatula, sp. conica; anfr. $61 / 2-71 / 2$, sat celeriter crescentes, ult. iufra attenuatus; ap. oblongo-ovata, callo par. tenui, marg. exter. leviter curvato, subrecto, fere tenui, colum. dilatato, reflexo. L. $16-19$, d. $6-7 \mathrm{~mm}$. (Marea). Index 1837. Ic. 1347. - Forma 1) elongata Kob. (Ic. f. 1318, t. longior (ad 221/2 $\mathrm{mm}$.$) , anfr. 8$.

N. cephalonicus Mss. T. oblongo-conica, irregul. rugosostriata, albida, striis et maculis impressis corneis, superne rufocornea, sp. elougata; anfr. 7, regulares, ult. attenuatus, sut. impressa, tota descendens; ap. ovalis, grisea, callo par. tenui, marg. exter. late labiato, vix patulo, colum. hreviter reflexo, curvatulo. L. 16, d. 5-6 mm. (Tns. Jonice, Albania, Herzegowina). Coq. Schl. 1859. Ic. f. 1358. - Forma 1) concolor. W. (Ic. f. 1359), t. major (1. $18 \mathrm{~mm}$.), concolor. (Bosnia, Scrbia).

Var. conemenosi Bttg. (Nachr. bl. 1885): t. major, latior, corneo-grisea, sut. albida, anfr. $7-7 \frac{1}{2} ; 1.15^{1} / 2-191 / 2$, d. $7-8 \mathrm{~mm}$.

N. ossicus Bttg. T. conico-oblonga, irregul. rugosostriata, striis maculisque corneis; anfr. $71 / 2$, lenti, ult. basi sub- 
gibbosus, sut. albomarginata, ad ap. lente ascendens; ap. ovata, fuscula, basi recedeus, marg. exter. vix expanso, latiss. labiato, colum. breviter reflexo. L. 13 , d. 5, ap. 1. $42 / 3 \mathrm{~mm}$. (Gracia, Ossa). Jahrb. mal. Ges. 1885 c. f. Ic. f. 566 .

Var. discolor Bttg. l. c.: t. minor, gracilior, multo tenuior, sp. magis convexo-turrita, anfr. $71 / 2-8$, perist. multo minus labiatum; l. $11-12 \frac{1}{2}$, d. $4-4 \frac{1}{2}$, ap. $1.33^{3 / 4}-4 \mathrm{~mm}$.

N. stokesi Bttg. T. exacte turrita, corneo-albida, obsolete luteostrigata, striatula; anfr. 8, medii fere plani, lenti, ult. major, basi tumidulus; ap. acuminato-ovata, fuscula, albolabiata, col. subconcava, superne contorto-plicata, marg. subexpansis, exter. ad sut. parum arcuato, colum. subreflexo. L. 17\% , d. 7, ap. 1. $6 \mathrm{~mm}$. (Grcecia, ins. Amorgo). Proc. Lond. 1885 c. f. (Bttg.)

N. carpathius Bttg. Præced. similis, sed oblongo-turrita, corneo-lutea, passim fusculo-strigata, ruguloso-striata, sp. convexo-turrita, anfr. 7, convexiores, celerius crescentes, ap. magis obliqua, late ovata, perist. non labiato, marg. colum. reflexo. L. 14, d. $5^{1} \frac{1}{2}$, ap. $1.5 \mathrm{~mm}$. (Grcecia, ins. Karpatho). L. c. 1885 c. f. (Bttg.)

N. krueperi Bttg. T. ovato-conica, infra ventrosa, solida, corneo-grisea, ruguloso-substriata, passim malleolata, sp. celeriter conico-acuminata; anfr. $6 \frac{1}{2}$, sut. albofilosa, ult. magnus, ventrosus; ap. ut N. boettgeri, sed albosublabiata, marg. colum. magis recedente, minus lato, col. magis torta. L. 14 , d. 7, ap. 1. 51/2 mm. (Grocia, m. Veluchi). Nachr. bl. 1888. (Bttg.)

N. cresius Bttg. T. perf, turrito-conica, coeruleo-alba, passim strigis punctisque griseis picta, striatula; anfr. $7-8$, regulares, perparum convexi, ult. vix attenuatus; ap. ovata, flavescens, marg. subsimplici, exter. subrecto, arcuato, colum. verticali, superne dilatato et reflexo. L. 19, d. $62 / 3$, ap. 1 . $61 / 2 \mathrm{~mm}$. (Grrecia). Proc. Lond. 1885 c. f.

Var. chin- Mts. (Gr. Moll. 1889 c. f.): t. pallide coerulescens, flavida v. albida, anfr. ult. basi sat convexus; 1. 15-19, d. $5 \frac{2}{3}-6 \frac{1}{2}$, ap. $1.51 / 2-6 \mathrm{~mm}$. (Ins. Clios et Samos).

Var. ymius Mts. l. c.: t. magis obesa, albida, anfr. ult. basi sat convexus; 1. 15-171/2, d. $7-8$, ap. 1. $6 \mathrm{~mm}$. (Ins. Symi).

Var. cous Mts. 1. c.: t. gracilior, præsertim infra angustior, ap. angustiore; 1. 15, d. 15, ap. 1. 5 mm. (Ins. Kos).

\section{(6.)}

N. montanus Drp. T. elongata, ovato-couica, irregul. striata, densiss. spir.-lin., fusco-olivacea; anfr. 7, lente regulares, 
sut. marginata; ap. ovata, ảcuta, perist. alboincrassato, expanso, marg. distantibus, exter. superne leviss. curvato, colum. parum dilatato. I. $15-16$, d. 6-7, ap. 1. 51/2 mm. (Europa media). Tabl. moll. 1801. lc. f. 41. - Forma 1) albina Ch., flavescenti-cinerea, pellucida; 2) veriegatr M. T., griseo-strigata; 3) major Rm. (Ic. f. 386), elongato-turrita, basi latior, anfr. 9, sat convexi, 1. 18-20, d. $71 / 2 \mathrm{~mm}$. (Carn., Carinth., Bavar.); 4) minor, 1. 12, d. $5 \mathrm{~mm}$.; 5) colorata Colb., brunnea, perist. subpurpuream (Belg.); 6) ventricosa Loc., obesa, breve conica, l. 14, d. $7 \mathrm{~mm}$. (Gall., Germ. occ.); 7) mosquensis Mil., parva, elongato-ovata, ap. angustiore (Moskwa).

Var. carpaticus Cl. (Mal, Bl. VIII): t. angustior, perforata, anfr. $61 / 2-8 \%$, convexiores, latitudine celerius crescentes, sp. altior, interdum turrita, sut. profundior; 1. 11, d. 5 $(14-15: 6-61 / 2 ; 20: 7) \mathrm{mm}$. (Rumelia, T'ranssylv.)

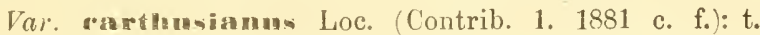
gracilior, cylindraceo-conica v. turrita, basi angustior, anfr. lenti, ult. longo angustoque, ap. angusta, latitudini testæ longior: 1. 15, d. 5 mm. (Gall., Bavar.)

N. calcasicus Pfr, T. subperf, oblongo-turrita, ruguloso-striata, densiss. spir.-lin. luteo-cornea (raro olivacea); anfr. $7-8$, convexiusculi, regulares, sut. albomarginata, ult. basi leviter attenuatus; ap. marginibus subapproximatis, alboincrassatis, callo nullo, exter. superne forte curvato, colum. verticali, perdilatato, reflexo. L. $14-17$, d. $6 \frac{1}{2}$, ap. $1.5-6 \mathrm{~mm}$. (Caucasus). Chemn. Cab. c. f. Ic. f. 2011. - Forma 1) major, 1. 1819, d. $7-71 / 2$, ap. $1.7 \mathrm{~mm}$.

N. ponticus Ret. T. anguste rimata, cylindrico-oblonga, sp. convexo-attenuata, apice mamillato, albida ad fuscobrunnea, striatula, striis spir. densiss. obsolete granulosa; anfr. 8-9, convexiusculi, lente regulares, sut. marginata, ult. basi rotundatus; ap. angusta, oblonga $v$. ovalis, callo tenuiss., marg. expanso-reflexis, alboincrassatis, exter. superne vix curvato, colum. late dilatato, col. plicata. L. $13-22$, d. $5-6 \mathrm{~mm}$. (Asia minor; Tauria). Mal. Bl. 1886 . Ic. f. 564.

Var. rugosus n.: t. forma, colore et sculptura microscopica ut typus, sed rugoso-striata, sp. paullo graciliore, pariete perobliquo, perist. valde albocalloso, callo pariet. crasso late dilatato, extns subtuberculifero; 1. 15, d. 5, ap. $1.5 \mathrm{~mm}$. (Tauria). Sub nomine B. turgidi Parr. misit Parr.

N. boettgeri $\mathrm{Cl}$. T. perf,-rimata, e basi subventroso conico-turrita, corneo-olivacea, striatula, distincte spir.-lin.; anfr. $7 \%$, sut. albomarginata; ap. perobliqua, late ovata, non labiata, callo par. nullo; marg. exter. superue valde curvato, colum. substricto. L. $12-15 \%$, d. $6-7 \%$, ap. 1. $6 \mathrm{~mm}$. (Transcaspia; Tauria). Mal. Bl. N. F. VI c. f.

$V a r$. connivens Bttg. (Jahrb. 1883 c. f.): t. solidior, corneo-flavescens, sp. brevius conica, anfr. $6^{1} / 2$, ap. albido-sublabiata, callo par. distincto, marg. multo magis approximatis; 1. 12, d. $6 \% / 2$ ap. $1.4 \mathrm{~mm}$. (Transcaspia). 
Var. oidogyra W. (Binn.-Conch., 1887): t. anfr. $7^{1 / 1}$, perconvexis, sut. profunda, ap. vix obliqua, oblonga, tenue labiata, marg. subæqualiter arcuatis, fere æque longis, valde approximatis, sejunctis; 1.13, d. $51 / 2$, ap. $1.4^{1} / 2 \mathrm{~mm}$. (Transcasp.)

N. obscurus Müll. T. ovato-oblonga, striatula, fuscobrunnea; anfr. $61 / 2-7$, sat celeriter crescentes; ap. rotundatoovata, perist. expanso, albolabiato, marg. colum. brevi, dilatato, callo par. nullo. I. 7 -- 9, d. $3 \frac{1}{2} \mathrm{~mm}$. (Europa). Verm. hist. 1774. Ic. f. 397. - Forma 1) limbate Loc. (Variat. Mal., 1880), perist. magis dilatatum et reflexum Gall.; 2) strangulata Loc. l. c., anfr. ult. humilior, cervice depressus, ap. angustior (Gall. ; 3) minor, Loc. l. c., t. miuor, anfr. convexioribus et sut. profundiore (Gall. '; 4) major, 1. 9--12, d. $5-6 \mathrm{~mm}$. (Gall.)

Var. perexilis Loc. (Conch. Fr., 1894): t. subcylindrica, gracile elongata, anfr. 8, sat lente crescentes, ap. callo par. tenui, marg. colum. elongato, leviter dilatato: $1.10-11$, d. $31 ;$ $-4 \mathrm{~mm}$. (Gallia).

Var. centralis Loc. 1. c.: t. breviter conoidea, infra ventrosula, anfr. 6, convexi, sat celeriter crescentes, nlt. convexus, ap. rotundata, callo par. nullo, marg. colum. curvato, reflexinsculo; l. 71/2-8, d. 41/2 $\mathrm{mm}$. (Gall.)

Var. columellaris W. (Binn. Conch., 1887): t. columella oblique ad sinistrum producta, crassa, callosa, marg. colun. sursum forte dilatato ac reflexo. (Helv.)

Var. oblougus IV. (Exp. crit., 1871): t. anguste rimata, oblonga, anfr. lentiss. crescentes, dno præultimi subæquales, ult. superne rectus, ap. parva. oblonga; l. 11 , d. $3^{1 / 2} \mathrm{~mm}$. (Snecia).

Var. dirae (Anc.) W. (Binn. Conch. Suppl. 1890; t. tenerrima, ap. subverticalis, perist. numquam labiato, marg. colum. paullo magis dilatato. (Algeria ad Aumale).

I'ar. Luubloerti B. (Amén. mal. 1I. c. f.): t. perf., cylindraceo-ovata, striatula, cornea, anfr. \&, convexi, regulares, ap. vix obliqua, parva, semiovata, perist. recto, col. recta; l. 9 , d. $4 \mathrm{~mm}$. (Tauria).

Var. unurosus Mss. (J. Conch., 1878 c. f.): t. rimata, ovato-oblonga, fuscocornea, sp. convexo-colica, anfr. $61 / 2$, planoconvexi, penult. maximus, nlt. paullo attenuatus, ap. sinuatoovalis, perist. expanso, tenue labiato, marg. colum. breviter dilatato; 1. 7-83/4, d. $3 \mathrm{~mm}$. (Transcancasia). Ic. f. 2036.

N. astierianus Dup. (Hist. moll., 1846 c. f.) T. perf., ovoideo-oblonga, infra medium ventrosula, fulva, sublævis; anfr. 6, perconvexi, regulares, sut. profunda; ap. ovata, parum excisa, perist. plano, albolabiato. L. 5-6. d. $2 \mathrm{~mm}$. (Gall., ins. Saint Marguerite). Dup. Similis Pupæ farinesii Desm. 
N. talyschanus Bttg. Differt a N. obscuro t. elongatofusif., sordide olivacea, passim strigis angustis pallidis, sp. gracili, acutiore, aufr. $71 / 2$, penult. quam ult. latiore, ap. minore, angustiore. L. 9, d. 3. ap. $1.2^{3} / 4 \mathrm{~mm}$. (Caucasus). Jahrb. 1880,1886 c. f. (Bttg.)

N. benjaniticus (Roth) Bens. T. perf,, elongato-ovato. conica, ferruginea, ad ap. pallida, subplicato-striatula, sp. sensim attenuata, sat gracili; anfr. 6, subconvexi, ult. subtus rotundatus; ap. ovalis, callo par. tenui, perist. recto, acuto, marg. colum. superne lato, expanso. L. $71 / 2$, d. $3,1.21 / 2 \mathrm{~mm}$. (Palast., Transcauc.) Ann. Mag. 1859. Ic. f. 2042. (Kob.)

N. martensianus Anc. T. rimata, conica, obtusa, striatula, e corneo pallide, violascens; anfr. $5^{1} / 2$ ult. basi rotundatus, saccatus; ap. ovata, perist. expansiusculo, tenui, albo, marg. approximatis, exter. valde arcuato, colum. dilatato, patente. L. 9, d. 5, ap. 1. 4 mm. (Turkestan). Bull. Fr. 1886. Ic. f. 591. (Mts.)

N. kuldshanus (Mss.) Mts. T. perf., conico-ovata, confertim striatula, corneo-albida, substrigata, sp. conica; anfr. 7, convexi, sut. tenui, profunda, superi celeriter crescentes, duo præult. turgidi, ult. supra planatus, ascendens, basi saccatus; ap. Iata, callo par. tenuiss., perist. undique expanso, tenue labiato, marg. exter. regul. arcuato. L. $9-10$, d. 5-6, ap. 1. $4 \mathrm{~mm}$. (Asia centr.) Centralas. Moll., 1882 c. f. Ic. f. 595.

N. coniculns Mts. T. subumbilicata, conica, striatula, superne corneo-fulva, infra albida, strigis raris latis violascentibus; anfr. $51 / 2$, convexi, sat celeriter et regul. crescentes, ult. basi angulatus, planatus, saccatus, superne ascendens, sut. profunda; ap. ovato-rotundata, perist. expansiusculo, tenuiusculo, albo, marg. approximatis, exter. valde arcuato, colum. dilatato. L. 6, d. 4, ap. $1.3^{1 / 2} \mathrm{~mm}$. (Asia centr.) L. c. 1882 c. f. Ic. f. 593 .

N. herzi Bttg. Differt a B. coniculo t. distinctins umbilicata et striata, apice acutiore, anfr. ult. rotundato, non ascendente, basi non angulato, ap. basi valde recedente, perist. simplici, tenui, marg. valde approximatis, exter. circulari, colum. e basi valde dilatato sinistrorsum protracto. L. $7-71 / 2$, d. $4^{1} / 2$, ap. l. $3 \mathrm{~mm}$. (Persia bor.) Zool. Jahrb. 1889 c. f. (Bttg.)

N. asiaticus (Mss.) Mts. T. oblongo-ovata, subtiliter striatula, corneo-fuscescens, concolor, sp. attenuato-conica; aufr. 
$7-71 / 2$, planiusculi, duo præult. subæquales, tumiduli, ult. planior et infra sensim angustatus, sut. superficiali; ap. truncatoovalis, subverticalis, callo par. distincto (interdum tuberculifero), perist. recto, albo, incrassato, marg. exter. recto, vix arcuato, colum. dilatato, reflexo. L. $8-15$, d. $4-5 \frac{1}{2}$, ap. $1.31 / 2-5$ mm. (Asia centr.) (onch. Mitth., 1882 c. f. Ic. f. 582.

Var. (eylindricocouns Anc. Bull. Fr. 1836): t. Inagis obesa et cylindracea, apice breve conica, anfi. 7, antepen. minns latus, ap. magna, subtrigonalis, col. incrassato-contorta, late ad basin oblique declivi; 1. 15, d. 7, ap. 1. $6 \mathrm{~mm}$. (Anc.)

Var. reseliaums Anc. 1. c.: t. ventricoso-ovata forma B. episoni B.', griseo-cornea, ad ap. flavescens, sp. a metlio obese ovali-conica, anfr. vix $6^{1} / 2$, ult. attenuatus, longior, ap. magna, intus griseo-albida, irreg. ovalis, callo par. nitido, perist. late expanso, planinsculo, albo, incrassato; l. 13. d. $6^{21 / 3}$, ap. 1. $6^{1 / 3} \mathrm{~mm}$. (Anc.)

N. komarowi Kob. T. rimata, eylindrico-ovata, summo breviter conico, luteo-fulva; anfr. S, plerumque super sut. impressam subimbricati, prominuli, ult. pone ap. planatus, ad basin attenuatus et compressus; ap. truncato-ovatib, basi recedens, callo tenuiss., marg. exter. recto, simplici, colum. verticali nou dilatato. L. $91 / 2$, d. 4, ap. $1.31 / 2$ mm. (Assia centr., Alai). Ic. f. 596.

N. subobscurus Anc. T. rimato-perf,, elongato-subovalis, fulvo-cornea, opaca, sp. conico-attenuata; anfr. $71 / 2$, ult. subattenuatus, convexus, antice subrectus; ap. semiovalis, col. arcuata, non callosa, nec alba, callo par. nullo, marg. exter. arcuato, recto, subsimplici, colum. expanso, dilatato. L. 91/2, d. $4^{2 / 3}$, ap. ad 3 mm. (4sia centr.) L. c. 1886 . (Anc.) Forma 1) fortior n., t. sut. obliquiore, arl ap. paullo ascendente, 1. $11^{1 / 2}$, d. $4^{1 / 2}$, ap. I. $3^{1 / 3} \mathrm{~mm}$. (Samarkand).

N. rufistrigatus (Bens.) Pfr. T. late striata, ovatooblonga, sp. oblongo-conica, albida, strigis maculisque rufocorneis; anfr. $7-8$, planinsculi, regulares, ult. leviter compressus; ap. oblonga, callo par. teuniss., perist. albo, labiato, expanso, marg. exter. sat curvato, colum. forte dilatato. L. 14-16, d. 6-7 mm. (Asia centr.) Hon. Hel. 1863. Ic. f. 1991.

Var. nuceyi n.: t. snbperf., periomphalo lato, ovatoconica, alba, nitida, strigis numerosis latis pallide brumneis, anfr. $6-7$, ult. basi rotuniatus, subtransversus, supra ascendens, ap. ovalis, callo par. distincto, perist. undique curvato et breviter expanso; l. $11-14$, d. 5 $1 / 2-6 \mathrm{~mm}$. (Turliestan).

N. sogdianus Mts. T. late perf, ovata, albida, strigis flavidis variegata, sp. convexo-conica; anfr. $61 / 2-7$, infra augulati, penult. turgidus, subgibbus, medii celeriter crescentes, ult. superne complanatus, basi rotundatus, saccatus; ap. ovato-rotun- 
data, ovalis, perist. subrecto v. leviter expanso et labiato, marg. conniventibus, callo par. tenui, tuberculo parvo. L. 12, d. 5-6 mm. (Turkestan). Moll. Turkest. 1874 c. f. Ic. f. 584. Forma 1) pericica (Yarr.), t. paullo brevior, albida, strigis translucentibus. (Persia).

Obs. Wh. Wuberyi Anc. (Bull. Fr. 1886), sub yuo nomine formas diversas accepi, etiam mihi species valde dubia vitletur.

N. potaninianus Anc. Differt a B. sogdiano t. multo majore et ventricosiore, rufostrigata et nebulosa, ap. magna, callo par. forti, medio abbreviato, perist. undique expansoreflexo, calloso, marg. magis approximatis, colum. dilatato. L. $16-16^{1 / 2}$, d. $71 / 2-8^{1 / 2} \mathrm{~mm}$. (Turkestan). L. c. 1886.

N. oxianus Mts. T. ovato-oblonga, albida, griseo-strigata; anfr. 6, regulares, ult. basi rotundatus, sp. subconica; ap. ovata, intus pallide flavescens, perist. breviter expanso, paullo incrassato, albo, marg. subapproximatis, exter. sat curvato, colum. subverticali, callo par. tenuiss. L. 14, d. 6, ap. l. $6 \mathrm{~mm}$. (Turkestan). Jahrb. 1876 c. f. Ic. f. 1993. - Forma 1) brevis $W_{\text {, }}$ t. strigis pallide griseis, sp. subovata, l. 11, d. $5^{1} / 2$, ap. 1. $5 \mathrm{~mm}$.

Par. sclualurudensis Bttg. (Zool. Jahrb.; 1889 c. f.): t. ninor, minus conoidea, regul. fusif., lateribus convexis, alba, strigis colneo-flavidis, anfr. $6-6 \% / 2$, plus minusve malleati, ap. callo par. distinctiore, extus fere incrassatulo; $1.10^{1 / 2}-12$, d. 5-6, ap. 1. 4-1'/2 $\mathrm{mm}$. (Persia).

N. Walteri Bttg. T. breviss, rimata, oblonga, cylindrata, albescens, passim strigis indistinctis diaphanis, sp. regul. fusif., lateribus convexis; anfr. 7, ult. quam penult. paullo longior, basi compresso-rotundatus, superne paullo ascendens; ap, intus hepatica, perist. breviter expanso, flavescente, marg. distantibus, callo par. tenuiss. L. $14--16$, d. $6^{1 / 2}-7$, ap. 1. $5^{1 / 2}-6$ mm. (Transcaspia). L. c. 1889 c. f. (Bttg.)

N. herzensteini Anc. T. anguste perf., elliptico-ovata, subobesa, apice conulum obtusum formante, subtenuis, cornea, strigis albidulis variegata; anfr. 7, ult. bene convexus, basi subtumidus; ap. perist. simplici, subrecto, marg. exter. parum curvato, colum. medio distincte calloso, arcuato, dilatato-expanso, callo par. indistincto. L. 11, d. $5^{1 / 4}$, ap. $1.4^{1 / 4} \mathrm{~mm}$. (Turkestan). L. c. 1886. - Forma 1) pellucens Anc. l. c., t. tenuiss., pellucida, cornea, fere concolor. (Anc.)

N. miser Mts. T. obeso-ovata, breviter striatula, pallide cornea $\nabla$. Alavida; anfr. $5 \frac{1}{2}$, vix convexiusculi, ult. basi rotundatus; ap. truncato-ovata, perist. recto, subsimplici, marg. distantibus, exter. arcuato, colum. dilatato, patente. L. 10, d. 5, ap. 1. $4^{1 / 2} \mathrm{~mm}$. (Turkestan). Moll. Turkest. 1874 c. f. 
Var. misellus W. (Ann. St. Petersb., 1896): t. multo gracilior, perf-rimata, oblongo-cylindracea, apice brevi, conico, nitido, irreg. obsolete striata, anfr. 6, sat celeriter crescentes, sut. impressula; $1.7 \frac{1}{2}$, d. $3 \mathrm{~mm}$.

N. przevalskii Anc. T. aperte perf., conoideo-turrita, alba, irreg. rufobrunneo-strigata, sublævis, sp. elongato-conica, convexa; anfr. 7, sut. impressa, ult. leviter ascendens, basi subsaccatus; ap. marg. distantibus, exter. recto, verticali, simplici, leviter curvato, colum. superne dilatato. L. 12, d. $4^{3} / 4$, ap. l. $3^{3} / 4 \mathrm{~mm}$. (Asia centr.) L. c. 1886. - Forma 1) minor, 1. 9, d. $31 / 2 \mathrm{~mm}$.

N. diplus W. T. ovato-conica, supra medium forte angusteque attenuata, cornea; anfr. 7, superi 4 striati, sat angusti, cæteri costati, sat lati, ult. basi rotundatus, superne ascendens; ap. ovalis, perist. incrassato, patulo-reflexo, marg. æque arcuatis, conniventibus, callo par. tenui. L. 11, d. $4^{1 / 2}-5$ mm. (Turkestan.) Ann. Ac. St. Petersb. 1896.

N. biformis W. T. anguste rimato-perf, cylindraceoconica, supra medium lente attenuata, apice rubro; anfr. $71 /_{2}$, superi regulares, medii sat fortes, ult. duo æquales, sut. usque ad ap. descendens; ap. oblongo-ovata, marg. distantibus, non conniventibus, callo tenuiss., exter. superne curvato, medio verticali, colum. superne patulo. L. 1l, d. $3 \mathrm{~mm}$. ('I urkestan). L. c. 1896 .

N. liostracus W. T. rimata, ovato-conica, sp. celeriter supra anfr. ult. conico-attenuata, lævigata, violascens, superne rufa; anfr. 6, perconvexi, sat forte crescentes, ult. basi rotundatus, superne leviter arcuatim ascendens; ap. intus flava, marginem versus late alba, ovalis, callo par. tenuiss., marg. exter. toto curvato, colum. subrecto, expansiusculo, basali arcuato. L. 10, d. 51/2, ap. 1. $4 \mathrm{~mm}$. (Turkestan). L. c. 1896.

N. goebeli W. T. omnino obtecte rimata, elongato-conica, sp. turrita, sat gracili, acuta, solida, lævigata, flavida, sub lente forti densiss. spir.-lin.; anfr. 7, sat regulares, ult. infra attenuatus, superne leviss. descendens; ap. oblique oblongoovalis, infra ad sinistrum subangulato-1'otundata, superne acutiuscula, marg. tenue alboincrassatis, nitidiss., exter. producto, longo, arcuato, colum. perbrevi, curvato, callo albo sub col. rectam intrante, callo par. tenui. L. 11, d. $4^{1} / 2 \mathrm{~mm}$. (Transcaspia ad Mangyschlak). L. c. 1896. 
(11.)

N. gibber Kryn. T. ovato-conica, paullo ruguloso-striatula, sub lente tenue spir. lin., coerulescenti-albida, sp. conicoturrita; anfr. 7--8, irregulares, sut. immersa, ult. convexus, elongatus; ap. ovalis, marg. exter. subrecto, subsimplici, superne forte arcuato, colum. brevi, dilatato. L. $18-22$, d. $7-8$, ap. 1. 6-7 mm. (Tauria). Bull. MIosc. 1833 c. f. Ic. f. 389 .

Yur. allomorplans, WV. (Binn. Conch., 1887): t. longior, cylindracea, sordide alba, anfr. 9, planulati, lenti, ap. semiovata, intus alba, nitida, perist. undique dilatato, labiato, marg. distantioribus; $1.26-27$, d. $8 \mathrm{~mm}$.

Var. plooreus B. (Amén. mal. 1860 c. f.): t. conicocylindracea, eleganter striatula, albida, nitens, anfr. $84 / 2$, convexi, regulares, ap. lunato-rotundata, nitida, marg. undique expansis, colum. paullo dilatato; 1. 25 , d. $8 \mathrm{~mm}$.

Ia). Cherouncesicus (Sow.) Jay (Cat. 1839: t. ovatooblonga, obsolete rugoso-striata, passim pallide strigata, sp. conico-turrita, ap. ovato oblonga, pallide aurantiaca, perist. simplici, paullo expanso; 1. 11-21, d. 8-9 $\mathrm{mm}$.

* L e u c o h i l o i des Pfr. (Nomencl. 1878).

L. loria Iss. T. rimata, elongato-ovata, striatula; anfr. $71 / 2$, ult. ventrosulus, leviter ascendens; apertura rotundata, marg. albis, exter. valde arcuato, colum. curvato. L. $6^{1} / 2$, d. 2, ap. 1. $2 \mathrm{~mm}$. (Persia, Tspahan). Moll. Pers. 1865 c. f.

L. samavaëusis (Mss.) Pal. T. rimato-perf., elongatoovata, lævigata, sp. convexo-conica; anfr. $5 \frac{1}{2}$, sat convexi, ult. subattenuatus, raptim superne breve ascendens; ap. lunato-semicircularis, perist. albido, plane expanso et reflexo, marg. remotis, exter. supra vix, iufra regul. curvato, colum. rectiusculo. L. $4 \frac{1}{5}$, d. $1^{3 / 10}$ (5: 21/2 Pal.) mm. (Mesopotamia, Samava). Ann. Genova 1872 c. f. (Mss. J. Conch. 1874).

L. eupliraticus B. Præcedenti similis, sed differt t. obese ovato-ventricosa, argutiss. striatula, sp. acuminato-elongata, ap. marg. sat approximatis, corneis, crassis, callo par. tenuiss., extus dentifero. L. $4 \frac{1}{2}$, d. $2 \frac{1}{2} \mathrm{~mm}$. (Mesopotamia, Samava). Sp. noviss. 1876. (B.)

I. reboudi B. T. perf., elongato-acuminata, subconoidea, luteo-cornea, apice obtusiss; anfr. 6, convexi, regulares, ult. rotundatus, ascendens, sut. impressa; ap. rotundata, vix lunata, perist. valido, plane expanso, acute reflexo, marg. valde approximatis, callo par. tenui, extus dentifero. L. 4, d. $13 / 4$ mm. (Algeria). L. c. 1876. (B.) 
* 0 m p h a 1 o c o n u S W. (Binn. Conch. 1887).

0. euryomphalus (Let.) B. T. irregul. costulato-striata; aufr. supra obsolete angulati, ult. forte dilatatus, circa umbilicum infuudibulif. in cristam obtusam compressus. L. $61 / 2, \mathrm{~d}$. 5 mш. (Kabylia). Sp. noviss. 1876. Ic. f. 2033.

* Retow ski a Bttg. (Nachr. blatt 1881).

$1 /$ schlaflii Mss. T. tenuis; anfr. ult. subcylindricus, inferne leviter attenuatus; ap. lata, irregul. ovata. L. $131 / 2$, d. 7, ap. 1. $6 \mathrm{~mm}$. (Transcaucasia). Coq. Schl. 1863.

Var. ingens Bttg. (Nachr. blatt 1888): t. multo major, exacte cylindrata, anfr. S/2, lentius crescentes, ap. angulo basali ad marg. colum. distinctiore; 1. $231 / 4$, d. $8^{3} / 4$, ap. 1. $91 / 4$ mm. (Cauc., m. Guk).

* II e d e a Bttg. (Jahrb. 1883).

M. carduchus Mts. T. pallide carnea, concolor, sp. crasse conica, obtusa, vix apertura longior; anfr. $51 / 2$, ult. subglobosus; ap. subcircularis. L. 19, d. 11, ap. l. $91 / 2 \mathrm{~mm}$. (Lurdistan). Vorderas. Conch. 1874 c. f. Ic. f. 1990. (Mts.)

II raddei Kob. T. lactea, zona lata fusca sub suturam, sp. turrita, forte acuminata, multo apertura longior; anfr. S, ult. oblongus; ap. ovata. L. 26, d. 13 , ap. $1.11 \mathrm{~m} . \mathrm{m}$ (Cancetsus). Ic. f. 2018-9. - Forma 1) minor Bttg., J. 151/2-17, d. $7 \frac{1 / 2}{2}$ $-8^{1 / 2}$, ap. 1. $6^{\mathrm{t} / 2}-7 \mathrm{r} / 2 \mathrm{~mm}$.

* H i.s t u s (Beck) Kob. (Cat. 1881).

Conspectus.

$\dagger$ Mustus s. str. T. Aextrorsa. (Algeria, Eur. merid. et Isia vecid.) (1).

tf Amphitrorsus Kim. (Transsylumia).

* T. dextrorsa. ( $(2)$.
** T. sinistrorsa. (3).

M. episomus B. T: cylindraceo-ovalis, ventricosa, apice prorsus attenuato-mamillata, dense decussata, pallide cornea; anfr. $7-8$, irregulares, superi convexi, ceteri plani, ult. infra attenuatus; ap. subrhombea, ad basiu angulosa, col, brevi, plicata, perist. labiato, expanso, marg. colum, dilatato. L. 16-20, 
d. 6-9 mm. (Cypria, Syria, Falcestina). Amén. mal. 1856 c. f. Ic. f. $1331-32,1334$.

Var. psendoepisomus B. l. c. (c. f.): t. apice lentius attenuata, anfr. regulares, col. recta ad marginem basalem tuberculifera. (Syria). Ic. f. 1333.

M. athensis (Friv.) Pfr. T. oblonga, sursum attenuata, striatula, cornea; anfr. 8-9, plani, lente crescentes, sut. albomarginata; ap. subrhombeo-rotundata, pariete parum obliquo, perist. expanso, albolabiato, callo par. crasso, tuberculo angulari transverso, marg. colum. dilatato. L. $20-22$, d. $61 / 2 \mathrm{~mm}$. (Turcia, m. Athos). Mon. 1848. Ic. f. 1345.

11. cretensis (Pfr.) Phil. T. cylindraceo-turrita, striata, carneo-lutescens; anfr. 7-8, sat celeriter crescentes, planulati, ult. tantum $2 / 5$ long. testæ, ad ap. breve descendens, sut. alba; ap. basi angulosa, pariete perobliqua, perist. forte labiato, subrecto, marg. colum. reflexo, callo par. pertenui, tuberculo parvo. L. $15-22$, d. $5-8$, ap. 1. $5-7^{1 / 2}$ mm. (Creta). Abb. $1845 \mathrm{c}$. f. Ic. f. 2012.

II. olivaceus (Pfr.) Phil. T. oblonga, irregul. costulatostriata, fortius infra suturam, ubi sæpe malleata, olivacea, sp. elongato-conica; anfr. 6-7, regulares, ult. $1 / 3$ alt. totius superans, superne breviter descendens; ap. ovata, tuberculo sæpe nullo, marg. exter. breviter expanso, forte labiato, colum. verticali, dilatato, patente. L. $11 \frac{1}{2}-15$, d. $5 \mathrm{~mm}$. (Creta, Andros). Abb. 1845 c. f. Ic. f. 2035.

II. dirphicus Bl. \& W. T. ovato-conica, apice acuto, regulariter densiss. striatula, brunneo- v. rufocornea; anfr. 8, regulares, sut. albomarginata; ap. ovata, basi angulosa, pariete parum obliquo, callo par. tenui, tuberculo forti. L. 19-24, d. 7 --8 mm. (Euboea). Apercu Gr. 1879. Ic. f. 2017.

M. milensis Bttg. T. conico-oblonga, obsolete striatula, nitida, lutea, strigis obsoletis fusco-olivaceis, sp. convexo-conica; anfr. 7, sut. lineari, crenulata; ap. parva, basi angulosa, pariete perobliquo, callo par. distincto, tuberculo nullo, marg. exter. infra extenso, colum. brevi, subreflexo. L. $181 / 2$, d. 8, ap. 1. $7 \mathrm{~mm}$. (Gracia, ins. Milo). Proc. Lond. 1885 c. f. (Bttg.)

M. kindermanni (Parr.) Pfr. T. obese v. elongate ovata, striatula, tenuiss. decussata, sordide carnea v. albida, sp. ovato-conica; anfr. 7, irregulares, planiusculi, sut. albomarginata; ap. basi angulata, anguste albolabiata, pariete parum obliquo, callo par. tenuiss., tuberculo fere nullo, marg. exter. recto, infra arcuato, colum. dilatato-patente. L. 15-17, d. $71 / 2-9$, ap. 1. $71 / 2-8 \mathrm{~mm}$. (Asia minor). Chemn. Cab. c. f. Ic. f. 1999. 
M. rossmässleri Pfr. T. oblongo-cylindracea, rugulosostriata, superne fusca, infra pallide cornea, subalbescens, sp. oblongo-conica; anfr. 7-8, irregulares, superi convexi, sut. albomarginata; ap. pariete parum obliquo, convexo, callo par. tenui, tuberculo forti, col. brevi, stricta, perist. breve patulo, albolabiato. L. 19, d. 7, ap. 1. 61/2 mm. (Asia minor). Proc. Lond. 1846.

Var. Ilavescens W.: t. irregul. obsolete striata, flavescenti-cornea; l. 12/ $/ 2-15$, d. $4 \frac{1}{2}-5 \mathrm{~mm}$.

II. anatolicus Iss. T. elongato-ovata, striata, corneobrunnea, sp. elongata; anfr. 8, sut. impressa; ap. callo distincto v. solummodo tuberbulo angulari, marg. exter. curvato, subrecto. L. $11^{1} / 2$, d. 5, ap. $1.5 \mathrm{~mm}$. (Asia minor, Armenia et Persia). Miss. it. Pers. 1865 e. f. Ic. f. 1995. (Mts.)

II. carueolus (Z.) Mbss. T. oblongo-ovata, apice attenuata, irregul. striatula, corneo-fuscula; anfr. 8, lente crescentes; ap. callo par. tenuiss., tuberculo distincto, perist. patulo, albolabiato. L. 12, d. $4 \mathrm{~mm}$. (Constantinopolis). Coq. Schl. 1856. Ic. f. 1365 - Forma 1) minor, anfr. 7, 1. $7 \frac{1}{2}-10$, d. $3 \frac{1}{2} \mathrm{~mm}$. (Græcia, ins. St. Georg).

M. subcarneolus B. Differt a B. carneolo t. fragili, tenui, ventroso-ovoidea, sp. breviter acuminata, anfr. 2 ultimis majoribus, ap. obliquiore, semirotundata, tuberculo angul. minimo v. nullo. L. 9, d. $4 \mathrm{~mm}$. (Turcia, Kalchi). Sp. noviss. 1876. (B.)

M. turgidus (Parr.) Kob. T. rimato-perf., ovato-oblonga, ventricosa, corneo-lutescens, regul. substriata, apice prorsus conico; anfr. $6-7$, regulares, ult. basi rotundatus; ap. pariete fere transverso, convexo, callo par. fere nullo, tuberculo valido, perist. expanso, labiato, col. superne forte plicata. L. 10-12, d. $5 \mathrm{~mm}$. (Groce. Archip.) Ic. f. 1357.

M. etuberculatus Frfld. T. ovato-oblonga, subconica, striatula, sub lente valido lineis spiralibus sicut granulata, virescenti-fusca; anfr. 7, lente regul. crescentes, sut. albida; ap. perist. superne recto, infra leviter expanso, intus leviss. labiato, callo par. tenuiss., tuberculo nullo. L. $13-15^{1} / 2$, d. $6 \mathrm{~mm}$. (Tns. Syra). Verh. sieb. Ges., 1867.

Il tetragonostona B. T. imperf, ventroso-oblonga, obsolete substriatula, lactescens, sp. convexo-acuminata; anfr. 7, lente regulares, inferi planulati; ap. oblongo-subtetragona, callo par. medio evanido, tuberculo valido, perist. candido, crasso, marg. colum. late expanso et depresso. L. 10, d. $4^{1 / 1}$ mm. (Bulgaria, Varna). Sp. noviss. 1876. (B.)

M. gastrum Ehrbg. T. rimato-perf, ovato-oblouga, utrinque angustior, ventrosa, fusco-olivacea, striatula, circa 
umbilicum lineis obsoletis spiralibus, sp. conica; anfr. 7, irregulares, sut. albida, vix obliqua; ap. semiovata, marg. exter. subverticali, callo par. pertenui, tuberculo sæpæ nullo, col. obliqua, brevi, labio tenui. L. $14-16^{1} / 2$, d. $6 \frac{1}{2} \mathrm{~mm}$. (Syria). Symb. 1831. Jahrb. 1882 fig. - Forma 1) minor, 1. 12-131/2 d. $7 \frac{1}{2}$ mm. (Syria, Turc. ins. Samothrake).

M. psendogastrum Hesse. Differt a præcedente t. latius perforata, pallide cornea $\nabla$. albescente, ap. rotundiore, marg. exter. magis curvato, fortius labiato, ad basin forte retuso, col. verticali. L. 15, d. $7 \mathrm{~mm}$. (Ins. Syra). Jahrb. 1882 . Ic. f. 1354.

I. uriæ Tr. T. cylindraceo-ventricosa, acute striatula, cornea v. corneo-olivacea; anfr. 7, ult. non $1 / 2$ long. testæ attingens; ap. obliqua, rotundata, perist. albolabiato, reflexo, marg. colum. vix dilatato. L. 15, d. 7, ap. 1. $51 / 2 \mathrm{~mm}$. (Palcestina). Proc. Lond. 1865. (Tr.)

M. pusio Brod. T. rimato-perf, ovato-globosa, apice breviss. conico, corneo-lutescens $v$. olivacea, irregul. striata, lineis tenuibus densiss. spiralibus; anfr. $5^{1 / 2}$, planiusculi, celeriter crescentes, sut. tenuis, subhorizontalis; ap. .pariete vix obliquo, callo tenuiss., tuberculo nullo, perist. expanso, subcrasse labiato. L. $12-13$, d. 7, ap. 1. $5 \mathrm{~mm}$. (Grocia, ins. Syra, Tino, Syphanto). Proc. Lond. 1836. Ic. f. 1356.

M. mestus W. T. ovato-globosa, striatula, pallide cornea v. lutescenti-albida, subdiaphana; anfr. 7, superi 6 lentissimi, ult. solus dimidiam testæ fere attingens, antice lente et sat alte ascendens, sp. ventricosa, superne in conum breviss. obtusum contracta; ap. brevis, semiovata, pariete subhorizontali, tuberculo angul. nodif., perist. expansiusculo, alboincrassato, callo par. tenuiss. L. $11-12$, d. $6 \mathrm{~mm}$. (Ins. Sofrano). Verh. Ges. Wien 1892.

II unius Bttg. Differt a. B. pusione t. majore, anfr. 6, striatulis, striis ad sut. non impressis, ap. tuberculo angul. transverso distinctissimo instructa et columella distinctius plicato-torta. L. 13, d. 8, ap. 1. $6 \frac{1}{2}$ mm. (Groecia, ins. Unio). Proc. Lond. 1885 c. f. (Bttg.)

II. papa Brug. T. rimato-perf, ovato-oblonga, irregul. striata, sordide lutescens, sp. conica; apice acuto; anfr. 7, irregulares, ult. major, infra attenuatus; ap. infra angustata, pariete obliquo, callo par. tenui, tuberculo forti, obliquo, spatio colum. lato, perist. anguste expanso, labiato, marg. colum. late reflexo. L. $13-14$, d. 51/2--6 mm. (Ital. merid., Sicil., Gracia, Smyrna, Algeria, Tauria). Encycl. 1792. Ic. f. (379) 711, 1351. - Forma 1) minor, t. ovata, 1. 10\% 1 , d. $4-5 \frac{1}{2}$ mm.; 2 ) etuberculata W., t. albescens, tuberculo ang. nullo; 3) bituberculata B., t. denticulo 
uno medio, altero angulari (Alger.); 4) substriatula B., t. cylindrica, sublævis, nitida, labio et tuberculo angul. validis; 5) primitiva Parr., t. infra albescens, superne brunnea, dense forteque striata, ap. marg. exter. leviter labiato, subrecto (Sicil.); 6) rentriosa Bttg. (Nachr. blatt 1894), t. 16, d. $7 \% / 2$, ap. 1. $7 \mathrm{~mm}$. (Gr., Cerigetto et Áchaia).

Var. obeso-sacuminatus B. (Mal. Alg., 1864): t. gracilior, sursum sensim attenuata, anfr. plani, ap. altior, perist. infre late expausum; 1. 16-18, d. $7 \mathrm{~mm}$. (Algeria, Napoli). Ic. f. 1353.

Var. grundis Mss. (Coq. Schl. 1859): ap. marg. exter. superne simplici, acuto, recto, sinuato, cretero forte nitiderue labiato, tuberculo angul. valido, oblique producto; 1. 17-20, d. $8 \mathrm{~mm}$. (Græcia).

Var. hrenbergi Pfr. (Proc. Lond. 1816): t. oblonga, apice attenuato, striatula, albida, anfr. $71 / 2$, ap. angulato-ovalis, callo par. crasso tuberculifero, marg. colum. dilatato, crasso; l. 20-2t, d. 10, ap. 1. $10 \mathrm{~mm}$. (Gr., ins. Cerigetto, subfoss.) Ic. f. 2000 .

I. meus (B.) W. T. oblongo-eylindracea, superne convexo-conica, apice perobtuso, lutescenti-albida, læviss. striatula, nitida; anfr. 7, irregulares, ult. parum major, infra rotundatus, vix declivis; ap. basi rotundata, pariete parum obliquo, callo obsoleto, tuberculo minimo, col. obliqua, acuta, perist. undique late expanso, labiato. L. 20-22, d. $9 \mathrm{~mm}$. (Anatolia). Binn. Conch., suppl. 1890.

IL hispalensis W. T. ovato-conica, flavido-cornea, irregul. obsolete striatula, sp. elongata, sensim attenuata; aufr. 8, sat regulares, ult. infra attenuatus; ap. pariete subhorizontali, callo par. tenui, tuberculifero, col, obliqua, perist. expansiusculo, tenue labiato, marg. colum. dilatato, reflexo. L. 14-16, d. $5^{1} / 2 \mathrm{~mm}$. (Hisp., Sevilla). Nachr. blatt 1892.

M. bielzi Kim. B. dirphico sintilis, sed t. virescenticornea, periomphalo iato, irregul. ruguloso-striata, sp. ventricosior, anfr. celerius crescentes, convexiores, sutura immersa, vix albicans, ap. pariete obliquiore, tuberculo minimo. L. 20, d. $8 \mathrm{~mm}$. (Transsylv.) Verh. siebenb. Ver. 1890.

Var. traxleri Kim. l. c.: t. latius rimata, gracilior, rufescenti-cornea, ap. fuscobrunnea, ad perist. alba, columella late pliciformi coarctata; 1. 17-22, d. 7- $8 \frac{1}{2} \mathrm{~mm}$.

I. transsylvanicus Kim. T. perforato-rimata, oblongoovata, irregul. striatula, cornea; anfr. 7-8, subregulares, superi magis, 3 inferi subæquales minus convexi, ult. basi subsaccatus; ap. pariete obliquo, callo par. medio tenui, tuberculo sæpe nullo, perist. patulo, labiato. L. $14-16$, d. $4^{1} / 2-6 \mathrm{~mm}$. (Transsylv.) L. c. 1883. Ic. f. 934. 
Var. temuis Bz. (Verh. sieb. Ver. 1860): t. angustiss. rimata, solidior, gracilior, subcylindrica, obscurior, anfr. convexiores, sut. profundior, labium crassius; l. 14-17, d. 4-5 mm. Forma 1) parva Bz., 1. 7-9, d. 31/2 mm.

\section{(3.)}

M. venerabilis Pfr. T. rimato-perf., cylindraceo-oblonga, præcipue extus ventricosa, apice forte acuminato, luteo-fuscula, sæpe rufobrunnea, dense striata, impressionibus spiralibus densis; anfr. 8-9, regulares, superi convexi, sut. impressa; ap. pariete parum obliquo, callo tenui, tuberculifero. perist. reflexo, patulo, labiato, col. obliqua, subcallosa. L. $16^{1 / 2}-21 \frac{1}{2}$, d. 7-81/2 mm. (Transsylv.) Chemn. Cab. 1853 c. f. Ic. f. 930 .

Var. alpicola Kim. (Verh. sieb. Ver. 1890): t. gracilior, spira lente attenuata; 1. $11-13$, d. $5-1 \mathrm{~mm}$. Ic. f. 931.

Var. regalis Kim. 1. c.: t. gracilis, clongata, subcylindracea, sericina, superficie granulosa, ap. ad plicas palatales bene promta; 1. 16-21, d. 5-7 mm. - Forma 1) alpina Bz., 1. $10-12$, d. $4 \mathrm{~mm}$.

Var. semotus Kim. I. c.: t. elongata, subcylindracea, cornea, dense striatula; l. 121/2-17, d. $4-5^{1} / 2 \mathrm{~mm}$.

Var. crassilabris Bz. (Verh. 1853, Ic. f. 933): t. minor, perist. crasse labiato, callo par. et tuberculo angul, nullis. - Forma 1) elongata Bz., t. gracilis, cylindracea.

\section{* Z e b r i n u s Held (Isis 1837).}

Conspectus.

$\dagger$ T. ventroso-ovata. (1).

tt $T$. breviter conico-ovata $v$. ventroso-oblonga. (2).

tt t T. ovato-conica $v$. cylindraceo-turvita.

*) Anfractus regulariter accrescentes.

$a$ - Apertura marginibus sopius disjunctis; $t$. alba, sape fusco-strigata; anfr. 7-8. (3).

b. - Apertura marginibus sapius conjunctis; t. cretacea; anfractus 9-12. (4).

**) Anfractus 3 ultimi suboquales; apertura marginibus tenue conjunctis. (5).

Z. spratti Pfr. T. rimato-perforata, ovato-acuta, sub epidermide olivaceo-lutescenti alba, solida, irregul. plicato-striata; anfr. $6 \frac{1}{2}$, sut. crenata, sp. conica; ap. altitudinem testæ fere rquans, truncato-ovalis. L. 21, d. $11 \mathrm{~mm}$. (Lycia). Phil. Abb. c. f. Ic. f. $1988,557$. 
Z. mirus W. T. latiuscule perf, elongato-ovata, ventricosa, albida, tenuiuscula, corneo-strigata, striatula, striis spiralibus interruptis clathrata; anfr. vix 6 , convexi, sut. non crenata; ap. ovata, longitudinem spiræ multo superans. L. 17, d. $91 / 2-10 \mathrm{~mm}$. (Syria). Binn. Conch. 1857. Ic. f. 558 (t. 18: $13 \mathrm{~mm}$.)

Z. subdetritus B. T' rimato-perf, breviter conoideoovata, obtusa, griseo-albida, obsolete strigata, striata; anfr. S, subconvexi, ult. magnus; ap. acute truncato-ovata, $1 / 3$ altitudinis testæ superans, intus pallide brunescens, callo par. tenui tuberculifero, marg. exter. superne leviter repando, albolabiato, colum. lato, reflexo. L. 14-15, d. $7 \mathrm{~mm}$. (Rumelia). Amén. mal. 1856 c. f. Ic. f. 917.

Var. bulgaricus B. (Sp. noviss. 1876): t. profunde perf., ventroso-oblonga, cretaceo-albida, passim corneo-flammulata, ap. semioblonga; l. 14, d. $6 \mathrm{~mm}$. (Bulgaria, Eidos).

Z. malleatus n. T. vix et breviss. rimata, obesa, oblonga, superne conica, luteo-albida, hepatico-strigata, obsolete striata, lineis spiral. ut densiss. longitudinaliter malleata; anfr. 7, fortes, regulares, subplani, ad sut. tenuem angulati; ap. ovalis, pariete et palato fusco-castaneis, callo par. albo minutiss. tuberculifero, marg. exter. ad basin anfr. penult. affixo, breviss. reflexo, labiato, colum. reflexo, aduato. L. 16, d. $61 / 2$, ap. 1. 61/2 mm. (Syria). - Num Kob. Ic. f. $1336 \mathrm{~g}$ (B. fasciol. var. e Cypria)? Zelebor olim ut fasciolatus mecum communicavit.

Z. hebraicus Pfr. T. rimato-perf., ovato-turrita, carnea, saturatius strigata, sp. elongata, acutiuscula; anfr. 7, planulati, supremi subtiliss. plicatulo-striati, corneo-maculati; ap. ovalis, intus pallide flavescens, marg. colum. dilatato, plano, patulo. L. 26-29, d. 12-121/2 mm. (Mesopotamia, Kurdistan, Asia minor bis Rhodus). Proc. Lond. 1851. Ic. f. 2007.

Z. detritus Müll. T. rimato-perf., ovato-conica, infra ventrosa, tenue striata; anfr. irregulares, penult. præcedente plus quam $1 / 3$ altior, ult. infra celeriter angustatus; ap. ovalis, basi angulata, intus luteo-corneola $v$. albida, marg. colum. dilatato et reflexo, col. acuta oblique descendente. L. 20 , d. 9-10, ap. 1. 9-10 mm. (Eur. media et merid., Caucasus, Asia minor). Term. hist. 1774. Ic. f. 42. - Forma 1) albida Mke. (Ic. f. 390), unicolor; 2) radicta Brug. (Ic. f. 1335 a.), t. alba, fuscostrigata; 3) unicolor Jan, t. cornea, perist. intus et sub. albis, raro albostrigata; 4) cornca Mich. (1. f. 391), t. tota cornea (Gall.); 5) lutea Z., t. lutea, fuscostrigata; 6) major, 1. 25-27, d. 11-13 mm. 
(Ic. f. 1335 c. d.); 7) minor, 1. 15-17, d. 7-8 mm.; 8) oblonga Blanc (lc. f. 1335 g.), t. ovato-oblonga, conica, 1. ad 27, d. $11 \mathrm{~mm}$. (It., m. Corno; 9 ' tumida (Parr.), t. medio ventricosior, sp. breviore, 1 . 24-29, d. $10-13 \mathrm{~mm}$. (Dalm., Epirus, Turcia \&c. ; 10) bavarica n. (Ic. f. 1335 b.), ap. intus brunnea (Regensburg); 11) parvula Næg., t. minor, tumida, fuscostrigata, 1.14 , d. $8 \mathrm{~mm}$. (Persia).

Var. nruouldi Fag. (Cat. Esera, 1887): t. subcylindraceo-conica, elongata, striatula, anfr. planiusculi, regulares, ap. anguste ovata, brevior; 1. 22-26, d. 10-111/2 mm. (Gall., Germ. occ., Helv., Triest). Fig. in Loc. Contr. 1881.

Var. subaudinus (B.) Loc. 1. c. c. f.: t. ut præcedens, at gracilior, anfr. irregulares, 5. quam superi major, ad sinistrum multo ventricosior, ult. infra elongatus, angustatus, sut. multo obliquior, ap. angusta; 1. 20, d. $8^{1} \frac{2}{2} \mathrm{~mm}$. (Gallia).

Var. crassus Ret. (Mal. Blätt., 1886: t. latius perf., ovato-conoidea, pallide cornea, sut. alba, sp. sat convexo-conica, ap. infra late rotundata, intus violascenti-cornea, marg. exter. medio forte curvato; 1.28 , d. $11^{1 / 2} \mathrm{~mm}$. (Tauria).

Z. fasciolatus Oliv. T. subrimata, oblongo-conoidea, striatula; anfr. planiusculi, subregulares, penult. præcedente non $1 / 3$ altior, ult. infra lente attenuatus; ap. augusta, basi rotundata, pariete perobliquo et palato intense castaneis, marg. colum. anguste reflexo. L. 15, d. $9 \mathrm{~mm}$. (Creta, Rhodus, Cypria, Grece. ins., Syria, Mesopot.). Voy. 1801 c. f. Ic. f. 910. - Forma 1) gracilis, cylindracea; 2) cundidus Pfr., t. alba, unicolor; 3) piochardi Heyn. (Nachr. blatt, 1870), t. . cornea, albomaculata. (Cypria).

Var. linrulistanus (Parr.) Pfr. (Mon. Hel., 1868): t. forma B. detriti, subovato-conoidea, magna, sp. conica; 1. 24 -26 , d. $10-11$, ap. 1. $9^{1} \frac{1}{2}-10^{1 / 2} \mathrm{~mm}$. (Kurdistan, Mesopot., Syria).

Z. hohenackeri Kryn. B. detrito simillima, sed t. latius rimato-perf., fere semper alba unicolor, sp. paullo convexiore, ap. late ovata, intus fusco-hepatica (pariete albo), basi rotundata, col. curvata. L. 26-28, d. $11-12 \mathrm{~mm}$. (Caucasus, Armenia, Kurdistan, Persia). Bull. Mosc. 1837. - Forma 1) minor, gracilior, 1. 20, d. 8-9 mm.; 2) fuscostrigata (Borshom, rariss.); 3) angulata W., sp. longa, turrito-conica, vix convexiuscula, anfr. infra ad sut. angulati, 1. 25-26, d. $10-11 \mathrm{~mm}$.

Z. dardanus Friw. ap. Pfr. T. subperf.-rimata, fusif.turrita, alba, quandoque superne fusculo-strigata, striatula, superne tenue spir.-lin.; anfr. sut. sat impressa disjuncti; ap. acute ovata, intus pallida, fulvida, pariete obliquo, marg. colum. late reflexo. L. 26-30, d. 9-11 mm. (T'auria, Constantinop., Asia minor). Hon. Hel. 1818. Ic. f. 905-906.

Z. eburneus Pfr. T. vix rimata, fusif-turrita, gracilis, candida, nitida, striatula, tenuiss. spir.-lin.; anfr. lenti, convexi, sut. sat impressa; ap. oblongo-ovata, acuta, intus dilute 
fuscula, pariete perobliquo, marg. colum. angustiss. reflexo. L. 23-26, d. 61/2-8 mm. (Mesopotamia, Syria). Symb. 1846. Ic. f. 907.

Var. renegatus n.: t. spira perlonga, anfr. supra medium planati, ad. sut. linearem angulati, ap. brevior. (Tarsus).

Z. cylindricus Mke. T. cylindracea, sursum lentiss. attenuata, alba, cretacea, striatula, sæpiss. dense spir.-lin.; anfr. $9-10$, regulares, lenti; ap. ovata, ad basin subangulata, callo par. tenui, marg. colum. recto, verticali, reflexo. L. $23-29$, d. 7-8 mm. (Tauria, Bessarabia, Ciscaucasus). Syn. 1830. Ic. f. 380, 1340-41. - Varr. sequentes omnes in Tauria.

Var. fusil orm is Mke. l. c.: t. major, elongata, subfusif., alba, unicolor $\mathrm{v}$. strigis brunneis obsoletis, anfr. 11-12; 1.27 -33 , d. $71 / 2-9$ (raro $371 / 10: 83 / 10) \mathrm{mm}$. Ic. f. 1342.

Var. obsoletus (Parr.) Mss. (Coq. Schl. 1863): t. obesa, oblonga, sat conica, alba, distanter brunneo-strigata, obsolete clathrata; 1. $20-25$, d. $6 \frac{1}{2}-8 \mathrm{~mm}$. Ic. f. 1313 .

Var. acuminatus Ret. (Mal. Blätt., 1884): t. regulariter acuminato-conica, alba, raro intense strigata, anfr. 10-11, planiusculi, distincte decussati, ult. parvus; 1. 191/2-27, d.561/2 (raro 29: 8) $\mathrm{mm}$.

Var. subacuminatus Ret. (Bull. soc. Mosc. 1888): V. acuminato. similis, sed to minus gracilis, pure calcarea, rariss. superne strigis nonnullis brunneis brevibus, anfr. $9 ! / 2-10 \% / 2$, spiraliter lineati, ap. labio ad marg. colum. distincte impresso; l. $21-28$, d. $6-71 / 2 \mathrm{~mm}$. (Caucas. ad Novorossisks). Ic. f. 565 .

Var. bettai Ch. (Cat. 1837): t. cylindrica, candida, nitida, lineis spir. nullis,. anfr. 11, ult. $1 / 4$ long. testæ; 1. 28, d, $64 / 2 \mathrm{~mm}$.

Var. fuscilabris Kryn. (Bull. Mosc. 1833 c. f.): $t$. cylindraceo-conica, candida, superne maculis parvis brunneis, obsolete clathrata, anfr. 9-10, perist. extus sæpe fusco-limbata; $1.17-22$, d. $6-61 / 2 \mathrm{~mm}$.

Var. turgidus Kryn. l. c.: t. abbreviata, obesa, magis conica, candida, nitida, vix spir.-lin., anfr. 8-9; 1. 17-22, d. $7 \mathrm{~mm}$.

Var. zebriolatus (Cl.) Ret. 1. c.: t. forma ut varr. 2 præcedentes, sed minor, brunnescenti-albida, forte brunneostrigata, anfr. $81 / 2-9$, ult. fere $1 / 3$ long. testæ; 1. 16-191/2, d. $6 \mathrm{~mm}$.

Var. illibatus Z. ap. Rm. (Ic. f. 381): t. ovali-oblonga, sp. attenuata, obtusa, anfr. $71 / 2-8$, ult. $2 / 5$ long. testæ, col. flexuosa, callosa; l. 16, d. $6 \mathrm{~mm}$.

T'ar. petropluius B. (Sp. noviss. 1876): t. aperte perf. elongato-oblonga, sat ventricosa, apice magno, obtuso, albida. 
striata, leviter malleata, anfr. 10, ap. oblonga, callo par. valido; l. 17, d. $6 \mathrm{~mm}$.

Var. cruzyi B. l. c.: t. perf., ventroso-oblonga, eleganter striata, albida, luteo flammulata, sp. ventricosa, superne rapide acuminata, anfr. $8 \frac{1}{2}$, ap. semirotundata, col. valida, recta; 1.18 , त. $8 \mathrm{~mm}$.

Var. Ienomphalus B. l. c.: t. aperte umbilicata, ventroso-conica, albida, grosse striatula, apice valido, anfr. 9, planulati, arctiss. crescentes, ap. fere semirotundata, callo par. tenui; 1.16, d. 8 mim.

Var. nuerejkowskii (B.) W. (Verh. Ges. Wien, 1892) t. ovato-conica, anfr. ult. parvus, planulatus, postice lente declivis, apice basali fere omnino in axi testæ posito; 1.20 , d. 6 , ap. 1. $5 \%$ $\mathrm{mm}$.

Var. limloutus n.: t. parva, cylindraceo-oblonga, griseofulvida, irregul. striata, supra medium lineis spiral. sicut dense granulata, passim strigis angustis fuscis ex limbo fusco aperturæ, anfr. $7 y,-S$, ult. postice lente declivis, ap. oblonga, acute truncata, col. superne gibbosa; l. $14^{t} / 2-15$, d. $4^{t} / 2-5$, ap. 1. $5 \mathrm{~mm}$.

Z. varnensis (Friv.) Pfr. T. fusif. cylindracea, superne attenuata, gracilis, lactea, nitida, sublrevigata; anfr. 10, ult. parvus, ad rimam subcompressus; ap. parva, intus fuscula, oblongoovalis, ad basin col. leriss. angulata, marg. colum. parum dilatato, patulo, exter. leviter curvato. L. $20-26$, d. $5-6^{1 / 2}$, ap. l. 51/2-7 mm. (Bulgaria). Mon. 1818. Ic. f. 1337-39.

Z. calverti B. T. imperfor., cylindrica, elongatiss., leviter acuminata, præsertim ad sut. striata, albidula, fuscoflammulata, anfr. 9, lente regulares, ult. convexo-rotundatus; ap. obliqua, semioblonga, intus subcastanea, ad basin col. valde angulata, subcanaliculata, perist. albolabiato, marg. colum. dilatato, reflexo et adpresso, col. exigua, recta. L. 21, d. 5-6 min. (Tns. Rhodus). Sp. noviss., 1876. (B.)

\section{Gen. B r e p h u I u s Beck (Ind. Moll., 1837).}

Conspcetus.

$\dagger T$. cornea, unicolor, temuis; ap. angusta, infra forte vetusa, marg. late listantibus, callo par. tenuissimo $v$. mullo, tuberculo any. parvo.

*) Col. callosa, tortuosa, in marginem colum. dentif. transiens; margo cxter. 1-2 dentatus.

1. T. dextrorsa. (1).

2. T. sinistrorsa. (2). 
**) Col. stricta, perist. simplex, marg. exter. intus valde labato, non dentato. (3).

it T. alba, sape strigata (raviss. cornea); ap. infra vix v, non retusa, marg. callo crasso conjunctis.

*) T. dextrorsa.

1. Ap. callo plicif. sutura paralleto in medio palati, marg. non dentatis. (4).

2. Ap. faucibus non plicata, marg. dentatis. (5).

**) T. sinistrorsa, cretaceo-candida. (6).

B. bicallosus (Friv.) Pfr. T. fusif.-cylindracea, a medio sensim attenuata; anfr. $10-11$, subplani, ult. postice plauatus; ap. dente 1 in medio marg. exter., perist. expanso, albolabiato. L. 20-21, d. 5, ap. l. $5 \mathrm{~mm}$. (Penins. Balkan). Zeitsch. f. Mal. 1847. Ic. f. 908. (Pfr.)

B. orientalis (Friv.) Pfr. T. fusif.-oblonga, corneo-lutescens; aufr. 10-111/2, planiusculi, angusti; ap. oblonga, dent. 2 in marg. exteriore (inferiore minimo), perist. rubello-labiato; L. $17-23$, d. $5-5^{1} / 2 \mathrm{~mm}$. (Asia minor, Ins. Prinkipos). L. c. 1847 . Ic. f. 909 .

B. frivaldskyi Pfr. T. cylindracea, utrinque attenuata, cornea; anfr. 9, planiusculi, regulares, ult. postice forte attenuatus, basi compressus, sut. albida, infra obliqua; ap. obliqua, anguste ovata. L. $14-15$, d. $4^{1} / 2$, ap. I. $5 \mathrm{~mm}$. (Brussa, Tns. Prinkipos). Mon. hel. 1848. Ic. f. 2002. - Forma 1) minor, 1. 10 -12 , d. $3-31 / 2 \mathrm{~mm}$.

Var. raynevalianus Raym. ap. B. (Amén. mal. 1856 c. f.): t. major, sub lente elegantiss. striatula, anfr. 10-11, convexiusculi, ap. perobliqua, angulato-ovata, marg. exter. subdentata, col. subconvexo-recta; 1. 15-17, d. $5 \mathrm{~mm}$. (Gallipolis). Ic. f. 2003.

Var. Ieptolenus B. (Sp. noviss. 1876): t. cylindrica, elongatiss., luteolo-cornea, anfr. 10, angusti, ap. semioblonga, perist. recto, acuto, labiato, marg. exter. superne sicut subdentifero; l. 14, d. $4 \mathrm{~mm}$. (Tauria).

B. attenuatus Kryn. T. gracilis, cylindracea, superne conica, nunc obsolete nunc forte rugoso-striata, albida, con- 
color v. fusculo-strigata; anfr. 9-10, lente regulares, planiusculi; ap. semiovata, perist. subrecto, incrassato, labiato, col. superne arcuata, crassata, semper a margine remota. L. 17, d. $4^{1} / 2$, ap. 1. $4 \mathrm{~mm}$. (Tauria). Bull. Mosc. 1833 c. f. Ic. f. 382. - Forma 1) minor, 1. 91/2-10, d. $2 \frac{2}{3}$ mm.; 2) appendiculatce Jan, t. gracillima, alba; 3 ) zebriolata Z., t. ventricosa, fuscostrigata; 4) fuscostrigata Bk., t. gracilis, fuscostrigata; 5) perdicea Z., t. ventricosa, alba, anfr. 8-9, 1. 11-16, d. 4-5 mm.; 6) clausitiaformis (Fér.) Mke, t. gracilis, callo in palato forti; 7) ecallosa n., t. sordide albida, faucibus omnino ecallosis, $1.14-15, \mathrm{~d} .4 \mathrm{~mm}$.

Var. theodosianus B. (Sp. noviss. 1876): t. ovato-turrita, solida, lævigata, lactea, nitida, rarius et sæpe tantum sub sut. immersam fuscostrigata, anfr. 8-9, ad sut. subangulati; 1. $18-19$, d. $6 \mathrm{~mm}$. - Forma 1) minor, $1.14-15$, d. 5 mm.; 2) major, 1. 22 , d. $6 \mathrm{~mm}$.

Var. costatus Ret. (Mal. Blätt. VI): t. anfr. 8-10, costis fortioribus ventricosior; 1. $8-15$, d. $3-3^{1} / 2 \mathrm{~mm}$. (Sudak).

B. duboisi Mss. T. ovato-cylindracea, fortiter striata, alba v. subradiata; anfr. 91/2 - 10, regulares, planiusculi; ap. ovata, subverticalis, plica palatali valida dentiformi, perist. recto late albolabiato, callo par. ad insertionem rectam interrupto, col. profunda oblique fortiter uniplicata. L. 16, d. 51/2 mm. (Transcaucasia). Moll. Schl. 1863. (Mss.)

B. biplicatus Ret. T. cylindrica, elongata, utrinque attenuata, solida, corneo-fusca, striatula, apice acutiusculo; anfr. $111 / 2$, convexiusculi; ap. elongato-ovalis, plica palatali valida, perist. expanso, late reflexo, albolabiato, callo par. dente ang. minimo. L. 20, d. $4 \mathrm{~mm}$. (Asia minor, Batum). Ber. Senck. Ges. 1889. (Ret.)

B. zebra Oliv. T. sæpe vix rimata, fusif.-cylindracea, albida, fuscostrigata, lævigata, nitida; anfr. 8-10, late regulares, planiusculi, ult. attenuatus, sut. lineari; ap. infra paullo retusa, angusta, lam. 1 par. et d. 1 parvulo nodif. ad medium marg. exter., col. recta v. superne inconspicua, infra dentif. producta, perist. fere recto, callo sæpe nullo. L. 14-15, d. $31 / 2-4 \mathrm{~mm}$. (Grocia, Ins. Rhodus, Cerigo, Rumelia, Brussa). Voy. Or. 1801 c. f. Ic. f. 653. - Forma 1) alba, unicolor; 2) obesa, l. 12, d. $4 \mathrm{~mm} . ; 3)$ tantrlus Parr., ap. subquadrata, infra dilatata; 4) brumnea Bttg. (Nachr. bl. 1894), t. brunnea, fere unicolor (1. Cerigo). - Varr. secuentes dentem palat. magis lamelliformem quam typus habent.

Var. spoliatus (Parr.) Pfr. (Symb. 1848): ap. dente colum. sæpiss. nullo v. rare minuto et $d$. marg. sæpe intus paullo elongato; 1 . $10-12$, d. $3-3 \frac{1}{2}$ mm., vel $15-17: 3 \frac{1}{2}-4 \frac{1}{2}$ mm. (Græcia). Ic. f. 1360. 
Var. olwsoletus Hesse (Jahrb. 1882): t. sæpius forte strigata, callus par. distinctior, lam. par. bene evoluta, sed.d. colum. minimus et d. marg. sæpiss. inconspicuus. (Gr., Lykobettos).

Var. leeoticus B. (Sp. noviss. 1876): t. lanceolato-elongata, cylindrica, sp. subacuminata, anfr. !, arcti, ult. dextrorsum sat provectus, ad basin compressiusculus, ap. 3-dentata, col. obliqua; 1.12 , d. $4 \mathrm{~mm}$. (Thebe).

l'ar. cualuoesums B. 1. c.: t. oblonga, cylindrica, anfr. 7, arcti, ult. subangulatus, ap. 3 dentata, col. recta; $1.10^{1 / 2}$, d. $4 \mathrm{~mm}$. (Thebe).

Var. lueliconirus B. 1. e.: t. cylindrica, elongatiss., anfr. 9, ult. convexus, basi rotundatus, ap. suboblongo elongata, 2-dentata, sc. d. colum. nullo, marg. colum. late expanso et rimam obtegente, col. recta; 1. 14, d. $4 \mathrm{~mm}$. (Boeotia).

Tar. fusitormis Desh. (Exp. Morée c. f.): t. fusif., alba, interdum fuscostrigata, sæe apice atro; 1. 14, d. $4^{2} \%$ mm. (I. Thermia).

I ar. nuatolicus Gall. (Bull. mal. Fr., 1884): t. aperte rimata, alba, unicolor, ap. labio crasso.

Var. zeloropsis B. ap. Gall. l. c.: t. longissima, cylindrica, anfr. sæpius 12, ult. ad rimam tenuem compressus, ap. perist. ad basin magis quam apud formas cateras expanso. (Brussa).

B. compactus (Friv.) Pfr. T. perf., oblongo-cylindracea, sursum lentiss, attenuata, albida, sat obsolete fuscostrigata, ruguloso-striata; anfr. 10-11, subconvexi, arcti, ult. lente attenuatus, sut. impressa; ap. oblonga, 3-dentata: lam. 1 par. immersa, d. 1 colum. obsoleto exserto, d. 1 marg. minimo, callo par. crasso, col. recta. L. $14-15$, d. 5-6 mm. (Creta, Brussa). Zeitsch. f. Mal. 1848.

B. olympicus (Parr.) Kob. T. aperte perf., cylindraceoturrita, sub lente striatula et dense spir.-lin., allida, brunneostrigata; anfr. 12, planiusculi, angusti, sut. lineari, ult. antice depressus, basi attenuatus; ap. oblonga, forte 3-dentata, callo par. crasso, marg. colum. leviter reflexo, col. recta. L. 15$18^{1} / 2$, d. 4-5, ap. 1. 4-5 mm. (Grocia et ad Brussa). Jahrb. 1877 e. f. Ic. f. 1361.

Var. chordatus n.: t. minor, distinctius eleganter spir.lin., anfr. 9-11, convexiusculi, sut. impressa, ap. distinctius ad basin coarctata; 1. 10-13, d. $2^{1} / 2^{-3} \mathrm{~mm}$. (Tauria, Koktebel).

B. bradus Gall. T. anguste perf, oblongo-elongata, striatula, omnino candida, sp. oblonga; anfr. 10, vix convexiusculi, perangusti, ult. sat exiguus, basi attenuatus, ad perf. valde angulatus; ap. exigua, infra sub-ovata, forte 3-dentata, perist. intus valde incrassato. L. $10^{1} / 4$, d. $3^{1} / 4$, ap. $1.2^{1} / 2$ mm. (Asia minor, Boyabad). Bull. ma]. Fr. 1884. (Gall.) 
B. bithynicus Gall. T. perf., oblonga, sat ventrosa, parum elongata, argute striatula, albido-lutescens, infra straminea, raro subflammulata; anfr. 9, angusti, ult. convexus, ad perf. compressus; ap. subovato-trigonalis, obsolete 3-dentata, d. colum. minimo, elongato, d. marg. exiguo, profundo, marg. exter. profunde labiato. I. $131 / 2$, d. 5, ap. 1. $4^{1 / 4} \mathrm{~mm}$. (Asia min., Déle-Tschaï). L. c. 1884. (Gall.)

1B. blanfordianus (Nev.) Kob. T. rimata, subcylindrica, ruguloso-striata, submalleata, sordide alba, sp. breviter conica; anfr. 9, planiusculi, regulares, ult. attenuatus, basi rotundatus, sut. marginata; ap. rotundato-ovata, perist. expanso, incrassato, marg. exter. medio tuberculo crasso marginali, colum. dilatato, profunde obsoliteque plicato. L. $18^{1} / 2$, d. $6 \mathrm{~mm}$. (Tersia). Ic. f. 2001. (Kob.)

B. tonruefortianus Fér. T. late rimata, subfusif., tenuiss. striatula, in anfr. ult. strigis paucis flavidis, sp. longa, lente turrito-attenuata; anfr. 11-12, planulati, ad sut. subangulati, superi lentiss., cæeteri regul. crescentes; ap. intus fulva, 3-dentata, dentibus crassis, lamellif., perist. subrecto, incrassato, callo par. subcontinuo. L. $22-27$, d. $6-7 \mathrm{~mm}$. (Constantinop., Brussa). Prodr. 1£21. Ic. f. 652. - Forma 1) cuplagiostoma Gall., ap. perobliqua (Boyabad).

B. ghedeoni Gall. T. rimata, subconoideo-oblonga, iufra sat tumida, argute striatula; anfr. 11, lenti, ult. magnus, sat tumidus, convexus; ap. subtrigonali-ovata, intus lutescens, 3dentata, dente marg. dentif., perist. recto, valide incrassato, marg. colum. dilatato, reflexo, callo par. valido. L. 23 , d. $71 / 2$, ap. 1. $71 / 2 \mathrm{~mm}$. (Asia minor, Tarakll). Bull. mal. Fr. 1884. (Gall.)

B. rivetianus Gall. T. anguste rimata, oblongo-acuminata, nitida, fere lævigata; anfr. 11, angusti, ult. exiguus; ap. trigonalis, intus pallide coffea, 3-dentata, dentibus exacte oppositis, lam. 1 par., d. 1 colum. et d. 1 marg. robustis, perist. recto, incrassato, marg. colum. obliquo, dilatato, callo par. tenui. L. $21 \%$, d. 6, ap. $1.5 \mathrm{~mm}$. (Asia minor, Boyabad). L. c. 1884 . (Gall.)

B. narcissei Gall. T. aperte rimata, elongato-ventrosa, obsolete striatula, sp. subacuminato-attenuata; anfr. 11, tumidoconvexi, angusti, sut. profunda, ult. convexus, basi coarctatus; ap. ovato-subtrigonalis, intus pallide luteo-castanea, valide 3dentata, d. colum. sat infero, perist. robuste labiato, patulo, marg. colum. dilatato, callo par. valido. L. $21^{1} / 2$, d. 7, ap. l. $61 / 2 \mathrm{~mm}$. (Asia minor, Boyabad). L. c. 1884. (Gall.) 
B. merloianus Gall. T. perf., cylindraceo-fusif., perelungata, fere lævigata; anfr. 12, arcti, sut. impressa, ult. parvulus, convexo-oblongus, extus provectus; ap. suboblonga, intus pallide castanea, 3-dentata, perist. valde incrassato, patulo, marg. callo junctis, colum. dilatato ac reflexo. L. 24 , d. $5^{1 / 2}$, ap. $1.6 \mathrm{~mm}$. (Asia minor, Boyabad). L. c. 1884. (Gall.)

B. becharianus Gall. T. rimata, infra relative tumida, medio cylindrica, elongata, ad summum attenuata, argute striatula; anfr. 12, angusti, ult. magnus, ventroso-tumidus; ap. obliqua, semiovata, infra sat amplo-rotundata, intus lutescens, valide 3-dentata, perist. labiato, ad col. dilatato-reflexo, callo par. distincto. L. 26, d. 7, ap. $1.7 \mathrm{~mm}$. (Asia minor, Snégueul). L. c. 1884 . (Gall.)

\section{Gen. C h 0 \| d r u l u s Beck (Index 1S37).}

Conspectus.

A. Chondrulus s. str. (Europa \& Asia occ.)

$\dagger T$. dextrorsa.

1. T. oblongo-ovata v. cylindraceo-turvita; ap. 1-2.dentata (rariss. edentata).

*) Ap. lam. 1 lavi in par. et d. 1 subolsoleto infra medium marg. exter. (Eur., Dalm.) (1).

**) Ap. semper $d .1$ in marg. exter., interdum etiam dent. in par. et col. lavibus. (Canc., Asia- (2).

2. T. ovato-oblonga, apice conico; ap. 3-(5-)dentata, dente mo alterove obliterato. (3).

3. T. oblongo-ovata; ap. lam. et dent. 4-6 (marg. colum. lam. forti v. dent. 2).

*) Ap. marg. exter. 1-dentato $v$. interdum etiam superne denticulis 1-2. (4).

**) Ap. marg. exter. dent. 2 vel lam. 1 et d. 1 vel lam. 2. (5).

4. T. orato-oblonga, subcylindracea, albida, sape fuscostrigata; ap. denticulis 1-4. (Cypria). (6).

5. T. ovata, ventricosu (rariss. oblonga); ap. lam. par. 2 (exteriore minore), d. colum. 2, d. marg. exter. 2-4, subpalat. (in Tabio remoto, crassiusculo). (7).

6. T. ovato-elongata, subcylindrica, sp. conico-attenuata; ap. lam. 4 validiss. fere clausa. (8).

7. T. cylindrica, elongata, apice breviss. conica; ap. 34-dentata. (9).

8. T. turrita, lente sursum attemuata.

*) Ap. 5-dentata $(2-1-2)$. (10).

**) Ap. 3-dentata (1-1-1). (11). 
9. T. conica; ap. 1-2-dentatu (dent. interdum obsoletis). (12.)

tf $T$. dextrorsa vel (rarius) sinistrorsa, subventroso-ovata; ap. 5-dentata (2-2-1). (13).

tit $T$. sinistrorsa.

1. T. oblique ventroso-ovata; ap. 6-llentata. (14).

2. T. anguste ovato-conica, oblonga; ap.6-dentata. (15).

3. T. ovato-oblonga, pallida; ap. 1-2-dentata. (16).

4. T. ommino cylindrica; ap. 3-dentata. (17).

5. T. oblongo-conica; ap. 2-lentata. (18).

6. T. cylindraceo-turrita; ap. 2-4-dentata. (19).

7. T. crasse cylindracea, apice rotundato; ap. 3-dentata, lam. par. pone ad marg. exter., sinulo perangusto. (Cuuc.) (20).

B. Chondrulopsis W. (Asia centralis). (21).

Ch. consentaneus (Z.) W. T. oblonga, apice conico, striatula, rufescenti-cornea; anfr. $71 / 2$, lente regulares, ult. antice planulatus, sut. albida; ap. ovalis, dente parrulo in medio par., perist. tenuiss. labiato, non expanso, marg. distantibus, callo par. diffuso, tenui, exter. leviss. curvato, leviter impresso, colum. dilatato. L. 10, d. $4 \mathrm{~mm}$. (Dalm.) Binn. Conch. 1887. Forma, magnitudine et colore persimilis B. obscuro, apertura B. diffuso.

Ch. lencodon Pfr. T. oblonga, tenue striata, obsolete clathrata, diaphana, cornea, sp. apice attenuata; anfr. 9, regulares, vix convexiusculi, sut. albomarginata; ap. oblonga, albolabiata, callo albo funiculato, marg. exter. expanso, supra medium dente valido, colum. lato, reflexo. L. 17, d. $6 \mathrm{~mm}$. (Asia minor). Phil. Abb. Ic. f. 2013. (Pfr.)

Ch. geoffreyi Anc. T. perf., tenue striata, cornea, sp. subelevato-elongata; anfr. 8, regulares, præsertim superi subrotundati; ap. ovalis, perist. incrassato, reflexo, callo par. ad margines incrassato, extus tuberculifero, marg. exter. ad medium dente valido, colum. dilatato, col. flexuosa. L. $8^{1} / 2 \mathrm{~mm}$. (Persia, Mazenderan). Et. moll. ined. 1882. (Anc.)

Ch. brevior Mss. T. cylindraceo-ovata, fusco-cornea, ad ap. albida, striatula, sp. convexo-conica; anfr. $7-8$, primi convexi; ap. subverticalis, late truncato-ovalis, perist. subexpanso, marg. distantibus, callo par. nullo, marg. exter. superne 
subsinuato, deiude late albolabiato, superne denticulo. L. 11, d. $5 \mathrm{~mm}$. (Armenia). J. Conch. 1876 c. f. Ic. f. 1364.

Var. viator n.: to pallide lutescenti-cornea, vix striatula, ap. callo par. nullo at tuberculo ang. punctif., dente in marg. exter. valido; 1. 8, d. $3 \mathrm{~mm}$. (Erivan). Sub nomine $\mathrm{Ch}$. anatolici Iss. e coll. I’xtel accepi.

Ch. lederi Bttg. T. cylindrata $\nabla$. cylindrato-oblonga, corneo-olivacea, striata, ad sut. rugulosa; anfr. $71 / 28$, planulati; ap. ovato-triangularis, fusca, basi attenuata; perist. sub sinulo fere rectangulo expansum, albolabiatum, callo par. tenuiss., raro subtuberculifero, marg. exter. omnino edentato, col. stricta. L. $10-10^{3 / 4}$, d. $3^{3 / 4}$, ap. 1. $3^{1 / 2}$ mm. (Swanetia). Jahrb. 1883 c. f.

Var. Iimis W. (Binn. Cọnch. 1887): t. elongato-ovata, apice conico, anfr. 7, celeriter sat regulares, ap. subtriangularis, infra ad sinistrum forte angustata, marg. late scjunctis, valde in equalibus, exter. superne leviter curvato, acuto, rect o, deinde forte labiato, denticulis $1-2$, colum. lato reflexo; 1. 14, d. $6 \mathrm{~mm}$. (Transcauc., Borshom).

Ch. tuberifer Bttg. T. cylindrata v. rarius cylindratooblonga, corneo-badia, distincte peroblique striata, nitens; anfr. $61 / 2-7^{1} / 2$, ult. antice carneo-rubellus; ap. truncato-ovalis, callo par. temuiss., interdum subtuberculifero; perist. simplex, obtusum, ad sinulum subletractum, rubello-labiatum, marg. exter. tuberculo acuto, colum. subreflexo, col. remota, planata, incurva. L. $81 / 2-91 / 2$, d. $31 / 2$, ap. 1. c. $3 \mathrm{~mm}$. (C'aucasus). Jahrb. 1879 c. f. (Bttg.)

Ch. boettgerianus Kob. T. rimato-perf., cylindraceooblonga, subtiliss. striatula, albida, sp. rufocornea, convexo-conica; anfr. 7-8, ult. leviter ascendens; ap. subtriquetra, dente par. profundo, tuberculo ang. minimo; perist. obtusum, alboincrassatum, marg. valde remotis, callo par. uullo, exter. vix curvato, colum. dilatato. L. $121 / 2$, d. 7 , ap. $1.5 \mathrm{~mm}$. (Caucasus?). Ic. f. 2025-26. (Kob.)

Ch. diffusus Mss. T. perf.-rimata, oblongo-ovata, striatula, alba $\nabla$. pallide cornea, sp. convexo-conica; anfr. 7, superi convexi; ap. ovalis, profunde in par. nunc lam., nunc dente, interdum absoleto; perist. non expansum, late et forte albolabiatum, marg. remotis, callo par. tenui extus incrassato, exter. rectiusculo, col. profunda, obsolete plicata. L. 10, d. $5 \mathrm{~mm}$. (C'aucasus). J. Conch. 1876 c. f. Ic. f. 2014.

Ch. tridens Müll. T. oblonga, brunneo-cornea, dense striatula; anfr. 6-8, regulares, ult. ad ap. flavidus, medio impressus; ap. semiovata, basi rotundata; lam. 1 par. mediano (sæpe tuberculo ang.), d. 1 colum. infero et d. 1 marg. exter, 
supero (sæpius superne denticulo minimo); perist. forte carneolabiatum, marg. colum. dilatato, reflexo. L. 12, d. 5, ap. 1.4 mm. (Eur. media et merid., Caucasus, Asia minor, Syria, Armenia, Persia). Verm. hist. 1774. Ic. f. 33. - Forma 1) minor, 1. $8-9$, d. $3 \frac{1}{2}$ mm.; 2) minima, l. $6 \frac{1}{2}-8$, d. $3 \mathrm{~mm}$., sæpius dentibus levibus, d. colum. obsoleto; 3) elongata W., t. cylindracea, elongata.

Var. unideututus Iss. (Moll. di Pisa 1866): t. fulva, diaphana, ap. d. par. tantum munita; l. 10, d. $4 \frac{1}{2} \mathrm{~mm}$. (Pisa).

Var. Inliciensis (W.) Cl. (Mal. Blätt. 1879 c. f.): t. forma, colore et magnitudine B. obscuro similis, ap. sæpius tantum dente forti par. et vestigio dentis colum., sæepe etiam dente marg., numquam d. ang., marg. sæpius disjunctis; $1.9-$ 10, d. $3 \frac{1}{2}-4 \mathrm{~mm}$. (Galicia).

Var. vicinus W. (Binn. Conch. 1887): t. subcylindrica, tenuis, perlucescens, d. colum. debili v. deficiente; 1.8, d. 3-4 $\left(8 \frac{1}{2}-10: 31 / 3\right) \mathrm{mm}$. (Transsylv.)

Var. podolicus Cl. (Mal. Blätt. 1880): t. parva et obesa, anfr. angustioribus, dentibus omnibus obsoletis, callo par. tamen distincto; 1. 9, d. $3 \%$ mm. (Podolia, Russia in Orenburg).

Var. microstomus Andr. ap. Kryn. (Bull. Mosc. 1833): t. perf., subovato-oblonga, turgidula, brunnescenti-cornea, irregul. striata, anfr. 7, tumiduli, ap. subtridentata, antice retusa, perist. reflexum, album; l. $4^{3} / 4$, d. $2 \mathrm{lin}$. (Polonia austr.) Kryn.

Var. migratus Mil. (Moll. Mosc. 1881): t. ovatooblonga, fuscocornea, forte striata, anfr. 3 superi aquales, 4 duplo altior, ult. $1 / 2$ long. teste, ap. rotundato-triangularis, d. ang. et d. marg. tuberculif. superne, perist. late labiato, extus albo; l. 11, d. $41 / 2 \mathrm{~mm}$. (Russia).

Tar. rayianus B. (Amén. mal. 1855 c. f.): t. ovoideoventrosa, anfr. ult. basin versus subcarinatus, ap. ovali-subquadrangularis, vix excisa, bidentata, perist. crassiusculum, basi sinuatum, marg. colum. reflexo; l. 13, d. $6 \mathrm{~mm}$. (Gallia).

Var. distans n.: t. ovoidea, breviss., ventrosiss., obtusiss., anfr. 5-6, convexi, ap. subovalis, magna, tridentata (d. colum. sæpe obsoleto), perist. expansum, incrassatum, marg. valde distantibus; 1. 7-8, d. $3 \frac{1}{2} \mathrm{~mm}$. (Gallia). Ch. obesus Loc. 1890.

Var. Arsuatus n.: t. ovato-conica, ap. fere semirotundata, 5-dentata; 1. 11, d. $5 \mathrm{~mm}$. (Transsylv.)

Var. Kubanensis Mss. (Cor. Schl. 1863): t. minor, ventricosa, castaneo-rufa, ad ap. alba, sut. albofilosa, ap. dentibus exiguis, precipue colum. obsoleto; 1. 11, d. $5 \mathrm{~mm}$. (Caucasus, Persia).

Var. eximins Rm. (Ic. f. 305, 722): t. major, elongatoovata, sp. convexo-suluturrita, superne conica, anfr. convexioribus, ap. ad basin col. subverticalem plus minus angulata, sæe subtrigonalis, dentibus cum angulari sepius forti 5 , majoribus, 
præcipue lam. par. magna, intrante, nec non denticulis $1-2$ marg. ad sinulum; 1. 14-17, d. 51/2-6 mm. (Eur. merid.-or., Caucasus. - Forma 1) marcida Bttg. (Moll. Tal. Geb. 1886 c. f.), sp. convexa, obtusa, dent. parum validis v. evanescentibus, l. $12 \frac{1}{2}$, d. $51 / 2$, ap. $1.4 \frac{3}{4} \mathrm{~mm}$. (Caucasus).

Var. cumeolus n.: t. elongata, gracilis, cylindraceoconica, sursum fere a basi lente attenuata, acuta, lateribus leviss. convexiusculis, anfr. lentissimi, ap. dent. typicis, at exiguis; 1. 13-15, d. $4^{1} \frac{1}{2}$, ap. I. $4^{1} / 2$ mm. (Caucasus).

Var. Iangei Bttg. (Ber. Offenb. Ver. 1.883 c. f.): $t$. omnium maxima, perf., ovato-oblonga, ventricosa, cornea, superne conoidea; anfr. lentius crescentes, ult. minus altus; ap. sicut eximius; 1. $151 / 2-201 / 2$, d. $6-8 \mathrm{~mm}$. (Syria ad Haiffa). Ic. f. 722 p. p. - Forma 1) imbellis n., ap. triquetra (marg. exter. extense curvato, patulo, colum. recto subverticali, reflexo), lam. par. perobliøua, d. ang. parvo, d. colum. basali minimo, d. marg. obliterato superne, perist. late alboincrassato, callo par. nullo; 1. $18-20$, d. $8 \mathrm{~mm}$. (Caucas.)

Ch. daImaticus (Klec.) W. T. ovato-oblonga, tenuiss. striatula, corneo-rufescens; anfr. 7, planiusculi, sut. marginata; ap. plica longius intrante infra finem superiorem lamellæ par. medianæ eburneæ, nec non plicula ad dextrum in par.; perist. valde eburneo-callosum, callo infra marg. exter. et in col. ad dentes incrassato, extus lutescenti-limbatum. L. sp. max. 14, d. 5, sp. min. 1. 11, d. $4 \frac{1}{2}$ mm. (Dalm. ad Imoski). Sv. Vet. Ak. Förh. 1881.

Var. klecuki W. (Binn. Conch. 1887): ap. lam. par. valida, longe intrante, medio geniculata, plicula par. ad dextrum tenuiss., brevi, col. valde callosa, edentata, marg. cxter. tenui, simplici, edentato.

Ch. rothi Pfr. T. ovato-oblonga, striatula, corneo et albido strigata, sp. elongata; anfr. 8, sat convexi, ult. antice albido et impresso, basi rotundato; ap. denticulo ang. minimo, altero profundo in par., dente transverso validiore in col., d. maximo in marg. exter., perist. albo, vix expansiusculo. L. $91 / 2$, d. $3 \frac{1}{3}$ mm. (Gracia). Mon. Hel. 1855. Ic. f. 2040.

Ch. blandus (Friv.) Pfr. T. oblonga, lævigata, oleonitens, corneo-brunnea, sp. ventrosula, in conum obtusum angustata; anfr. 8, ult. basi compressiusculus; ap. trigono-ovata, d. 1 elato linguef. in par., 1 lamellif. in col. et 1 marginali, perist. albolabiato, vix expansiusculo, marg. subrqualibus, disjunctis. L. $10^{1} / 2$, d. $4 \frac{1}{3}$ mm. (Asia minor, Anasia). Zeitschr. f. Mal. 1853. (Pfr.)

Ch. quinquedatus (Mhlf.) Rm. T. cylindraceo-oblonga, sursum convexiusculo-conica, persimilis ( $\%$. eximio Rm., a quo 
præcipue differt ap. marg. exter. superne forte exciso, d. secundo par. minimo sub d. angularem, d. 2 in col. (superiore re vera basis col. contorte callosæ), d. 2 marg. superne, infero crasso, obliquo, lam. par. magnam subtangente. L. 12, d. 5$6 \mathrm{~mm}$. (Dalm., Triest, Bosnia, Caucasus). Ic. f. 301. -- Forma 1) major, gracilior, l. 18 , d. $5 \frac{2}{3} \mathrm{~mm}$.; 2) tumidas, W., l. 13 , d. $5^{2} / 3$ mm.; 3) uniparietalis Bttg., d. supero par. deficiente (Dalm., Caucas.) - Var. approximatus Pfr. - Sibiria. Nomen. (Pfr.)

Ch. bergeri Roth. T. perf., fusif.-ovata, lateribus convexis, a medio sursum ovato-conica, striatula, lutescens v. conea; aufr. 8, planulati, regulares; ap. ovata, basi rotundata, perist. late forteque alboincrassato, dent. 4 validis: 1 ang. obliquo, 1 par., 1 colum. transversali (ut in quinquedent.), 1 marg. opposito horizontali intrante. L. $14-16$, d. $6 \mathrm{~mm}$. (Penins. Balkan, Brussa, Alexandria). Moll. Sp. 1839 c. f. Ic. f. 721. - Forma 1) obesa W. (Binn. Conch. 1897), t. parva, ventrosa 1. 10, d. $4-4^{2} / 3 \mathrm{~mm}$; 2) euboice Reeve (Kob. Ic. f. 2016 ), t. elongata, cylindraceo-oblonga, sp. superne conica, lateribus minns convexis, sut. allomarginata, l. 14-16, d. $41 / 2-5$ mm.; 3) turrita WV. (l. c. 1887), t. longa, fusif., lateribus subrectis, superne conica, anfr. 10, perlenti, ap. elongata, ad basin angustata, l. 16, d. 41/2 mm. (Morea).

Ch. kollyi Ret. T. cylindrica, corneofusca, striatula, ad ap. late albida, nitida, apice turrito-conico; anfr. 9, planiusculi, ult. ad ap. medio impressus; ap. oblongo-ovalis, tuberculo ang. nullo, d. I par. profundo, 1 colum., 1 marg. conico; perist. albolabiatum, marg. basali et colum. expanse reflexis. L. 16, d. $5^{3 / 10}$ mm. (Asia minor, Batum). Ber. Senck. Ges. 1889. (R.)

Ch. microtragus (Parr.) Rm. T. cylindraceo-ovata v. subcylindracea, apice conico, cornea $\nabla$. albido-flavescens, tenuiss. obsolete striatula; anfr. $7-81 / 2$, lente regulares, antepen. et penult. æquales, ult. ad ap. medio forte impressus; ap. tuberculo ang. punctif., lam. par. magna, intrante, d. colum. lamellif., horizontali ad basin col. latæ et callosæ, d. marg. submediano, oblique transverso et intus sursum cum labio calloso, 1-2-denticulato adhærente; perist. crassolabiatum. L. $81 / 2-11, \mathrm{~d} .3^{2} / 3$ -4 $1 / 2$ mm. (Penins. Balkan, Tauria). Ic. f. 651. - Forma 1) major, l. $13-15^{1} / 2$, d. $51 / 4-51 / 2 \mathrm{~mm}$.

Var. unutatus W. (Binn. Concl. Suppl. 1890): ap. marg. basali interno non tam lato et recto, sed curvato, d. colum. altinus sito; 1. 12 , d. $5 \frac{1}{4} \mathrm{~mm}$. (Euboea, Aliveri).

Cl. montandoni (Desch.) W. Præcedenti similis, sed t. tenuissime, dense leviterque at regul, et eleganter striata, cinerascenti-albida, anfr. regul. celeriterque crescentes, antepenult. et penult. valde inæquales, ap. dente colum. ad basin col. sito, d. marg. fere supramediano. L. 12, d. $4 \frac{1}{2} \mathrm{~mm}$. (Dobrudscha, Baba-Dagh). Verh. Ges. Wien 1892. 
Ch. tricuspidatus Kstr. T. cylindrica, apice attenuato, obtuso, nitida, lævis, pellucida, cornea; anfr. 7; ap. lam. 1 in par. et perist. 3-dentato, albo, reflexo. L. $4^{1} / 2-5$, d. $2-21 / 3$ lin. (Syria, Beiruth). Chemn. Cab. c. f. (Kstr.) - Species dubia, nimis sequenti similis fide cl. Kob.

Ch. Ievaillantianus B. T. ventricoso-cylindrica, acuminata, albido-carnea, elegantiss. striatula; anfr. $7-71 / 2$; ap. lam. 1 par. magna, alta, contorta, intrante, d. 2 fortioribus ad basin col., d. 1 margin. exter. superne et sæpius tuberculo ang.; perist. valde alboincrassatum, paullo reflexum, callo par. tenui. L. 11, d. 5--6 mm. (Turcia, Adrianopolis). Amén. mal. 1856 c. f. Ic. f. 2022. (B., Kob.)

Ch. tetrodon Mort. T. cylindraceo-ovata, apice conicoobtuso, lutescens, substriata, nitjda; anfr. $7-8$, ult. basi rotundatus; ap. labiata, tuberculo ang. parvo, lam. l par., col. obliqua, remota, serrato-bidentata et marg. exter. recto medio unidentato, marg. colum. reflexo. L. $14-16$, d. $6 \mathrm{~mm}$. (Armenia). Mém. Inst. Gen. 1854 c. f. Ic. f. 918.

Ch. ghilanensis Iss. T. cylindraceo-oblonga, fulvocornea, apice conico, sub lente striatula; anfr. $71 / 2-8$, superi angusti, convexi, sut. marginata impressa, 2 præult. lati, subæquales, minus convexi; ap. ovata, lam. par. 1 brevi, superne denticulo adjacente, plica colum. 1 transversali superne, d. 2 marg. exter., subconicis, infero majore, raro etiam denticulo minutiss. extus in par. et plica tenui subverticali angulari; perist. albolabiatum, marg. fere æque arcuatis, distantibus. L. $81 / 2$, d. $31 / 2-4 \mathrm{~mm}$. (Persia, Caucasus, Transcasp., Syria; Tauria). Moll. Pers. 1865 c. f. Ic. f. 1994. - Forma 1) minor Bttg., t. multo gracilior, anfr. $7-7^{1} / 2$, ap. minor, l. $7-71 / 2$, d. $3 \mathrm{~mm}$.

Ch. isselianus (B.) Iss. Præcedenti persimilis e descriptione originali et figuris, differt solummodo t. grisea, anfr. 7, lente regul. crescentibus, ap. marginibus callo utrinque tuberculifero junctis. L. 8, d. $4 \mathrm{~mm}$. (Armenia). L. c. 1865 c. f. (B.)

Ch. libanicus Næg. T. parva, subovato-conica, cornea, striatula, sp. convexo-conoidea; anfr. 51/2, regulares, ult. superne horizontalis, basi rotundatus; ap. semiovata, 4-dentata: lam. 1 par. sat immersa, pl. 1 colum. horizontali, d. 2 marg. exter. rotundato-obtusis, infero majore; perist. incrassatulum, album, reflexiusculum, marg. distantibus, callo et tuberculo ang. nullis. L. 5, d. 21/3 mm. (Asia minor, Tibanon). Nachr. blatt 1897. Ex descr. rev. Nægelii l. c. "t. cylindrico-ovulif., corneo-brunnea, anfr. $6-61 / 2$, ult. compressus; ap. plicis 4 : I pl. par. robusta, 1 colum., 1 in marg. exter. et dente 1 superiore in insertione parvulo gibbulo, marg. callo junctis, l. 6, d. 3 mm." 
Ch. parreyssi Pfr. T. ovato-oblonga, apice conico, striata, albida, corneo-variegata; anfr. $61 / 2$, medii subplani, ult. infra ascendens, sut. subfilosa; ap. semiovata, tuberculo ang. punctif., denticulo profunde in par. immerso, col. remotiss., stricta, callosa, basi truncata, angulata, perist. patulo, labio crasso, albo, gibbosulo. L. 10, d. $4^{1} / 2 \mathrm{~mm}$. (Cypria). Symb. 1846. Ic. f. 924 .

Var. framdulentus W. (Binn. Conch. 1887): t. minor, ventrosa, col. valde callosa. - Ic. f. 925.

Var. truquii (Bell.) Mss. (Coq. Bell. 1854): t. coerulescenti- alba, nitida, strigis corneis angustis, ap. tuberculo ang. oblongo, transversali, denticulo par. profundo, obsoletiss., marg. exter. intus late subdenticulato, perist. albo utrinque aque ac late limbif. planerque cxpanso: 1. 13-15, d. $15 \mathrm{~mm}$. Ic. f. 2024.

Ch. limbodentatus Mss. T. oblongo-ovata, apice obtuse conico, striatula, carneo-alba, nitida; anfr. $6 \% / 2$ ult. antice subrectus; ap. late truncata, ovata, tuberculo ang. sæpe obsoleto, d. 1 par., d. 1 colum. parvo, d. 2 marg. exter., perist. forte labiato, callo par. tenui. L. 10, d. $4 \mathrm{~mm}$. (Cypria). Coq. Bell. 1854 c. f. (Mss.)

Var albreviarus Mss. l. c.: t. minor, ovata, sut. albomarginata, ap. fere semiovata, dentibns ap. distinctioribus. (Syria).

Ch. alumnus (Parr.) Pfr. T. vix rimata, ovato-oblouga, apice obtuse conico, corneo-lutescens, substriata; anfr. 7, ult. demum subascendens; ap. triangulari-semiovata, denticulis singulis minutis in angulo et profunde in par.; perist. labio crasso plano, marg. colum. breviss., replicato. L. 17, d. $41 / 2 \mathrm{~mm}$. (Cypria). Mon. Hel. 1848. Ic. f. 926. (Rm.)

\section{(7.)}

Ch. septemdentatus Roth. T. oblongo-ovata, ventricosa, superne conica, corneo-lutescens, irreg. striata; anfr. $61 / 2-7$, superi lenti, medii forti, ult. antice subconstrictus, supra subplanus, basi subsaccatus; ap. late truncata, semiovata, d. ang. cum lam. supera par. parvula interdum confluente, lam. secunda par. inmersa, d. 1 colum. medio, transverso. d. 1 minore ad basin, d. 2 marg. subæqualibus, perist. labiato, reflexo. L. 8-11, d. 4-5 mm. (Syria, Palcestina, Ḱnudistan). Moll. Sp. 1839 c. f. Ic. f. 322 . - Forma 1) muxima 13., t. fuscocornea, antice alba, rugoso-striata, labio et dentibus valiclis, $1.10-15$, d. $4^{1} / 2-5^{1} / 2$, $\mathrm{mm}$.; 2 ) borealis MIss. (J. Conch. 1874), t. gracilior, sut. sepe albomarginata, ap. minus rotundata, dent. brevioribus, l. 9-10 $\mathrm{mm}$. (Syria); 3) elonyata (Roth) Mss., t. ovato-oblonga, fusca, dent. validis. 
Var. albulus Mss. (Coq. Roth 1861): t. conico-ovata, nitidiuscula, anfr. subconvexi, superi pallide cornei, ult. brevior, albescens, ap. parvula, dent. invalidis; $1.8-9$, d. $3 \frac{1}{2}-4 \mathrm{~mm}$. (Jerusalem).

Var. rugulosus n.: t. ovata, ventricosa, apice perbrevi et forte acuminato-conico, sub lente ruguloso-striata, pallide cornea, anfr. 7, superi convexi, perangusti, cæteri (præestim penult.) lati, convexiusculi; l. 9, d. $5 \mathrm{~mm}$. (Jerusalem).

Ch. triticeus $\mathrm{Rm}$. T. præcedenti similis, sed ovali-subcylindrica, apice conoideo-rotundato, nitida, læviuscula, anfr. 6 . ult. deorsum attenuatus, basi compressus, ap. tuberculo ang. minimo libero. L. $9-10$. d. $4 \frac{1}{2}$ m $\mathrm{mm}$. (Palcestina). Ic. III. (1859) c. f.

Ch. lamelliferus $\mathrm{Rm}$. T. parva, rimato-perf., oblongoovata, acutiuscula, striatula, albida v. cornea; anfr. 6, sat regulares, ult. major, antice striatus, infra ad ap. scrobiculatus, basi compressus, superne bene ascendens; ap. semirotundata, tuberculo ang. parvo, lam. 1 par. valida, longe intrante, medio tortuosa d. 1 colum. transverso, superne, d. 1 basali parvo, d. 3-4 marg., superis minimis, perist. breviss. reflexiusculo, tenue labiato. L. $6^{1} / 2$, d. $3^{1} / 2-4 \mathrm{~mm}$. (Syria). Ic. f. 919 .

Var. plıasianus Dub. ap. Mss. (Coq. Bell. 1854): t. late perf., breviter ovata, medio ventricosa, supra breviter conica, anfr. ult. sæpe valde ascendens, æque striatulus, convexior, basi rotundatus, lam. par. sæpius cum tuberculo ang. confluens, perist. fortius labiatum; 1. $5^{1 / 2}-6$, d. $2^{1} / 2-3 \mathrm{~mm}$, (Transcaucasia). Ic. f. 2028. - Forma 1) minor, 1. 4, d. 21/2 mm.; 2) major, 1. 7, d. $3 \frac{1}{2} \mathrm{~mm}$.

Var. angustatus Ret. (Mal. Blätt. 1886): t. elongatoovata, sp. superne conica, anfr. 7, ap. extus depressa, lam. par. parum tortuosa, cum tuberculo conjuncta, d. in medio marg. exter. subquadrato; $1.5 \frac{1}{2}$, d. $2^{2 / 3} \mathrm{~mm}$. (Tauria).

Ch. angustior Ret. Differt a præcedente $t$. obiongoovata, fere duplo minus lata, corneo-fusca, sp. magis at lentius attenuata, apice acutiore, tuberculo ang. parvulo, libero, lam. par. minus sigmoidea, d. $2-3$ marg. inferis, subæqualibus, superiore punctif. L. $41 / 2-51 / 4$, d. $21 / 4-21 / 2 \mathrm{~mm}$. (Caucasus). Bull. Mosc. 1888.

Ch. ovularis Oliv. T. perf., ovata, apice breviss. attenuato, pallide cornea $v$. lutescens, subtiliss. striata; anfr. 51/2 -6 , subconvexi, penult. multo quam antepenult. altior, ult. ascendens, basi subsaccatus; ap. semiovata, forte dentata, dent. fere ut $C h$. septemdent., sed d. marg. profundius in labio lato intus elato sitis et denticulo par. magis immerso. L. $4 \frac{1}{2}-5$, d. $3-3 \frac{1}{2} \mathrm{~mm}$. (Asia minor). Voy. Ottom. c. f. Ic. f. 920.

Var. sulcidens Mss. (Coq. Roth 1861): t. paullo minor, corneo-hyalina, dentibus non conicis, apice rotundatis, sed clavæformibus, summo bipartitis. (Asia minor, Aegyptus). 
Ch. brotianus (Cl.) Kob. T. rimato-perf, elongatoovata, apice regul. attenuato, subtiliss. striatula, albida, summo flavido; anfr. 7, lente regulares, subconvexi, sut. profunda, ult. basi compressus, non ascendens; ap. tuberculo ang. parvulo, lam. 2 par. inæqualibus et tertia minima, pl. 1 colum. horizontali et d. 1 basali; perist. dilatum sed vix reflexum, crasse labiatum. L. 9 d. $31 / 2 \mathrm{~mm}$. (Syria). Ic. f. 606. (Kob.)

Ch. didymodus Bttg. Ch. septemdentato similis, sed t. sæpius multo minor, magis cylindrata, tuberculo ang. punctif., dentibus geminis par. inter se perapproximatis, adeoque interiore superne hamiformi, d. basali sæpius evanescente, interdum duplicato, marg. colum. magis stricto. L. $71 / 2$, d. $3 \mathrm{~mm}$. (Caucasus, Armenia, Persia). Jahrb. 1880, 1886, c. f.

Var. cullilabris Bttg. (Zool. Jahrb. 1889 c. f.); t. major, magis cylindrata, anfr. $T \% / 2$, ap. longior, dent. marg. crassioribus, hebetioribus, basali calloso-evanido, marg. exter. convexo, latiss. albocallaso; $1.9-10$, d. $4-4^{\frac{1}{1} / 2} \mathrm{~mm}$. (Persia).

Ch. clessini Ret. T. perf., ovata, apice obtuse conico, subtiliss. striatula, cornea; anfr. 6-7, regulares, convexi, sut. impressa, ult. major, bene ascendens, antice pone basin impressus, basi subcompressus; ap. semiovata, lam. 2 par., interiore validiore, immersa, exteriore distante, tenui, sæpiss. cum insertrone marg. exter. conjuncta, d. 1 colum. lamellif., basali 1 parva, d. 2-3 marg., perist. expanso, albolabiato. L. 4-61/2, d. $21 / 2-32 / 3 \mathrm{~mm}$. (Asia minor; Tauria). Mal. Blätt. 1883 c. f. Ic. f. 602 .

Ch. heptodon Mts. T. cylindraceo-turrita, albida; anfr. 7, superi 5 regulares, flavescentes, 2 præult. subæquales, ult. infra rotundatus; ap. 7-dentata: lam. 2 par. (1. humili, subduplicata, 2. forti compressa), d. 1 colum., d. 3 marg. (supero minimo), perist. alboincrassato. L. 10, d. $4 \mathrm{~mm}$. (Aegyptus). Sitz. ber. Berlin 1885. (Mts.)

Ch. nucifragus (Parr.) Pfr. T. subperf., elliptico-ovata, medio ventricosa, corneolutescens, striatula, sp. conica; anfr. 6 , ult. turgidulus, deorsum attenuatus; ap. semiovata, tuberculo ang. nullo v. minimo, lam. l par. brevi, imınersa, d. l colum. transverso, immerso, forti, d. 1 basali parvulo, d. 2 marg. approximatis, intus in laloio extus declivi, parvis; perist. patulum, col. superne verticali, valida. L. $71 / 2-10$, d. $4-5 \mathrm{~mm}$. (Cypria). Mon. Hel. 1848. Ic. f. 921.

Ch. stylus (Parr.) Pfr. T. perf., globoso-conica, subtiliss. striata, nitida, lutescenti-alba; anfr. 5, convexi, ult. turgidus, antice ascendens; ap. d. 2 par. perapproximatis (exter. minimo), d. 1 in medio col., d. 1 basali ninimo, d. 2 conti- 
guis sursum et deorsum in callum palati tenui decurrentibus, perist. reflexo, marg. colum. breviss. L. 6, d. $4 \mathrm{~mm}$. (Cypria). IIon. Hel. 1848. Ic. f. 923.

Ch. nanus (Ret.) W. T. elongata, subcylindrica, apice obtusato, lutescenti-cornea; anfr. $71 / 2-8$, regulares, ult. 2 subæquales, ult. medio sulco horizontali, postice forte descendens, basi subsaccatus; ap. semiovata, lam. 4 validiss. fere clausa: 1 par., superne latiss., deflexa, cum marg. exter. contigua, intus geniculata et profunde intrante, 1 colum. horizontali, 1 basali brevi, late truncata, 1 marg. subquadrata, verticali in medio et superne ad sinulum perangustum 1 plicula; perist. late albolabiatum, marg. distantibus. L. $10^{1} / 2-11$, d. $31 / 2$, ap. 1. $3 \mathrm{~mm}$. (Smyrna; Tauria). Binn. Conch. 1887. Ic. f. 605.

Ch. arctispira Mss. T. cylindrica, sublævis, nitida, albida, sp. superne celeriter attenuata; anfr. $9^{1 / 2}-10$, superi celeriter, cæteri lentiss. crescentes, angusti, subplani, ult. vix major, sut. subplana, alba, marginata; ap. lunato-ovalis, lam. 1 par. forti, d. 2 colum. minutis, d. 1 marg. supra medium, marg. subparallelis, basali arcuato. L. 12-14, d. 41/2 $\mathrm{mm}$. (Mesopotamia). J. Conch. 1874.

Ch. euxinus Ret. T. late cylindrice perf., exacte cylindrica, apice breviter conico, griseo-cornea, nitens, subtiliss. striatula; anfr. 11-12, plani, lentissimi, ult. basi rotundatus, pone ap. sulco subtili; ap. lam. 1 par., d. 1 colum. horizontali, d. 1 marg. in medio, perist. expanso, albo, callo par. tenui, extus tuberculo interdum lam. par. conjuncto. I. 10-11, d. $3^{3} / 4-4$, ap. 1. $31 / 2 \mathrm{~mm}$. (Transcaucas.; Tauria). Mal. BIätt. 1883. Ic. f. 604. (Ret., Kob.)

Ch. sieversi Mss. T. rimato-subperf., cylindracea, striatula, pallide cornea, apice subito diminuta; anfr. $81 / 2$, inferi 4 subæquales, minus convexi, ult. lente ascendens, antice impressus, basi attenuatus et compressus; ap. ovato-trigona, crasso-labiata, lam. 1 par. parvula, retracta, lam., colum. antice nodulosa, d. 1 marg. maximo, pyramidali. L. 8, d. $2^{7} / 10 \mathrm{~mm}$. (Transcauc.) J. Conch. 1873 c. f. Ic. f. 2027 . (Mss.)

Var. Hoplites W. (Binn. Conch. Suppl. 1890): t. tenuiss. striatula, anfr. ult. basi rotundatus, ap. tuberculo ang. plicif. v. intus furcato, lam. par. profunda, breviss., alta, dentif., d. colum. forti, nodif. cum col. valida arcuata subconjuncto, d. marg. typico; $1.7 \%$, d. $3 \mathrm{~mm}$. (Persia ad Selmas.) 
Ch. sexdentatus Næg. T. turrita, striolata, cornea v. flavescens, sp. elongata, apice subacuto; anfr. $71 / 2$, regulares, sut. obliqua, ad ap. paullo ascendente, ult. postice lente descendens, ad basin subsaccatus; ap. obliqua, tuberculo ang. forti et 5-dentata: lam. par. crassiuscula, extus denticulo adjacente, pl. colum. transversa et d. 2 sublamellif. in marg. exter., perist. incrassato, albido. L. $8-91 / 2$, d. $3 \mathrm{~mm}$. (Ghajü, Libanon). Nachr. Blatt 1897.

Ch. mygdonicus W. T. aperte perf., subfusif,, sursum lente longeque attenuata, corneo-albicans, striatula; anfr. $9^{1 / 2}$ lenti, superi couvexi, sut. impressa, ult. postice planatus, albidus, circa periomphalum infundibulif, carinato-angulatus, infra transversus, superne prorsus descendens; ap. obliqua, tuberc. ang. parvo, lam. 1 par. brevi, denticulo 1 fere obsoleto infra col. et denticulo $1 \mathrm{marg}$. supra medium, perist. forte alboincrassato, late plano-expanso, callo par. tenuiss. L. $91 / 2$, d. $3 \mathrm{~mm}$, (Grocia ad Saloniki). Nachr. Blatt 1893.

Ch. munitus W. T. perf.-rimata, subfusif., sursum longe et lentiss. in conum acutiusculum attenuata, corneo-brunnea, densiss. obsolete striatula; anfr. 10-11, lenti, superi convexi, ult. infra lente attenuatus, antice impressus; ap. lam. 1 par. longa, humili, 1 colum. superne, transversa, sursum arcuata, d. 1 marg. transverso lamellif. in medio, perist. iucrassato, callo par. valido. L. 14, d. $31 / 4 \mathrm{~mm}$. (G'reecia in $\mathrm{m}$. Taygetos). L. c. 1893.

Ch. westerlundi Kob. T. perf.-rimata, turrita, elongata, lente attenuata, striatula. cornea, strigis albidis raris obsoletis; anfr. 9, lenti, ult. basi rotundatus, antice subplanatus; ap. subverticalis; lam. 1 par., lam. 1 colum. transversa et d. 1 marg. vix majore, perist. albo, vix expansiusculo, callo par. tenuiss., extus tuberculifero. L. $10^{1} / 2$, d. vix $3 \mathrm{~mm}$. (Grcecia). Ic. f. 2041. (Kob.)

Ch. kutschigi Kstr. T. rimata, conica, cylindracea, subtiliss. striata, corneo-flava, nitida, sp. alta, conica; anfr. 7, lenti, angusti, convexi, ult. convexus, basi rotundatus, sut. profunda; ap. semiovata, dente 1 lamellif, in par., perist. incrassato, albolabiato, callo tenui, extus dentifero. L. $71 / 2$, d. $3 \mathrm{~mm}$. (Dalmatia). Chemn. Cab. 1845 c. f. Ic. f. 2023. (Kstr.) 
Ch. diodou Ret. T. perf.-rimata, elongata, sursum gracile attenuata, acuminata, striatula, cornea, nitida; anfr. 7-9, regulares, ult. postice convexiusculus, pone ap. planatus, basi rotundatus, sut. marginata, impressa; ap. ovalis, 2-dentata: d. 1 par. compresso, minore (interdum deficiente) et d. 1 marg. multo fortiore, col. superne conspicua, obliqua, perist. albo, leviter incrassato, callo par. tenuiss. L. $6-91 / 2$, d. $21 / 2-31 / 4$ mm. (Tauria). Mal. Blätt. 1883 c. f. Ic. f. 603.

Var. mieans n.: t. nitidiss., anfr. 8, lentiss., omnes convexi, sut. profunda; l. 7. d. $2 \frac{1}{2} \mathrm{~mm}$.

Ch. pindicus W. T. subperf.-rimata, oblonga, sursum lente et convexiusculo attenuata, obtusa, calcareo-albida, solida, sæpe strigis brunneis, obsoletis; anfr. 7, lenti, ad sut. impressam subangulati, ult. postice et ad basin rotundatus, superne ascendens; ap. 2-dentata ut diodon, sed d. marg. minore, tilberculo ang. minuto et perist. crasso. L. $7-8$, d. $2_{1 / 2}^{1 / 3}$ mm. (Grocia, m. Pindus). Nachr. blatt 1893.

Ch. dubius Ret. T. rimata, gracile elongato-oblonga, tenue striatula, cornea, nitidula, sp. turrita; anfr. $71 / 2-9$, lente regulares, ult. postice depressus, antice planulatus, basin versus attenuatus; ap. ovato-oblonga, marg. exter. superne arcuato, supra medium subtuberculato, perist. expanso, albolabiato, col. bene conspicua, callo par. tenuiss. L. $61 / 2-11$, d. $2 \frac{1}{2}-31 / 2$ mm. (Tauria). Nal. Blätt. 1886. Ic. f. 607 .

Cl. cespitum Mort. T. sublævigata, læete cornea; aufr. 7, lenti, ult. basi gibboso-compressus; ap. subtriangularis, d. 5 valde inæqualibus: d. 1 plicif., oblique intrante ad insertionem, lam. 1 par. profunda cum primo subcontinuo, d. 1 plicif. profundiss. in col. et d. 1 marginali parvo ad medium col. nec non d. d. 1 maximo obliquo in marg. exter., perist. incrassato. L. 9, d. $4 \mathrm{~mm}$. (Armenia ad Ispir). Mém. Gen. 1854 c. f. Ic. f. 2043. (Kob.)

Ch. saulcyi B. T. rimato-perf., ovata, latere exter. multo convexiore, apice breviter acuminuto, striata; anfr. $61 / 2$, planulati, ult. basi compressiusculus, transversus; ap. 6-dentata, dentibus lamellif.: 1 ang.(tenui, superne bigibbo), 1 par. (valido, introrsum tortuoso), 2 colum. (superiore majore), 2 marg. (validis, distantibus), perist. albolabiato. L. 9-101/2, d. $4^{1} / 2-5 \mathrm{~mm}$. (Syria). Petit Journ. 1853 c. f. Ic. f. 918. 
Var. impressus Mss. (Coq. Roth 1861): t. minor (1. 7, d. $3 \mathrm{~mm}$.), elongato-ovalis, marg. sinistro extus impresso, dent. colum. minus approximatis, d. infero marg. exter. retro plicam longiss. emittente. (Jerusalem).

Ch. chondriformis Mss. T. rimato-perf., conico-elongata, levigata, oleaceo-cornea, sp. conoidea, acutiuscula; anfr. $7 \%$ convexi, ult. ascendens, basi subcompressus; ap. ovatotriangularis,tuberculo ang. et 6.dentata: 2 par. (supero minuto, mediano magno lamellif.), 2 colum. (basali obsoleto), 2 marg., distantibus et requalibus, intus in plicas longas exeuntibus, perist. albo, vix labiato. L. 7, d. $2^{4} / 5 \mathrm{~mm}$. (Jerusalem).

IIoll. Roth 1861.

Ch. botterianus Phil. T. ovato-oblonga, obtusa, striatula, corneo-albida, nitida; anfr. 7, convexi, ult. basi gibbus; ap. semiovalis, d. 1 parvulo profunde in par., perist. subrecto, crasse labiato, callo par. tenui. L. 9, d. $4^{1} / 3 \mathrm{~mm}$. (Dalm. in Lesina). Abb. c. f. Ic. f. 2021. (Pfr.)

Ch. incertus Ret. T. elongato-ovata, apice conico, striatula, cornea; anfr. 8, sat celeriter crescentes, ult. ascendens, basi leviter attenuatus; ap. 2-3-dentata: d. 1 par. lamellif., d. 1 ad basin col. (sæpe obsoleto), d. 1 marg. in medio, perist. recto, tenuiss. labiato, callo par. nullo. L. $7-11$, d. $3-41 / 2 \mathrm{~mm}$. (Asia minor, Samsun; Tauria). Mal. Blätt. N. F. VI. Ic. f. 1105.

Var. proprius n.: t. major, anfr. 9, regulares, sed lati, ult. antice non ascendens, basi attenuatus, ap. basi angustata, callo par. tenui, dente basali nullo, col. ad basin producta; 1. $13^{1} / 2$, d. $5 \mathrm{~mm}$. (Persia ad Tokat).

Ch. sagax (Friv.) Pfr. T. cylindraceo-oblonga, striatula, carneo-albida, sp. elongata, sursum convexo-conoidea; anfr. 8, lente regulares, ult. basi rotundatus; ap. vix obliqua, d. 1 profundo parvulo in par., perist. subrecto, albolabiato, callo par. tenuiss. subtuberculifero. L. $11-13$, d. $5 \mathrm{~mm}$. (Asia minor, Amasia). Mal. Blätt. 1858. Ic. f. 2006. (Pfr., Kob.)

Ch. mouradi (Desch.) W. T. cylindracea, superne conica, albida, striatula; anfr. 8 regulares, ult. antice rectus, basi non attenuatus; ap. ovato-trigona, denticulo 1 minuto in par. et interdum vestigio denticuli in marg. exter., perist. recto, parum incrassato, marg. colum. dilatato. L. $11-13^{1} /_{2}$, d. 4-41/2 mm. (Asia minor, Angora). Verh. Ges. Wien 1892. Ic. f. 1104 . 
Ch. ponsonbyi W. T, omnino cylindrica, elongata, apice perbrevi obtuse conico, lævigata, cornea; anfr. 10, lentiss., ult. 4 subæquales, superi convexi, cæteri subplani, ult. antice rectus, basi convexus; ap. sinistrum versus forte intensa, triangulari-semiovalis, 3-dentata: lam. 1 par. cum callo valido compresso ang. confluente et dent. transversalibus 1 ad basin col. arcuato-callosæ, 1 in medio marg. exter., perist. tenui, alboincrassato, late planeque expanso. L. 14, d. $4^{1} \frac{1}{2}$ $\mathrm{mm}$. (Lycia, Horzum). L. c. 1892 . Ic. f. 1103.

Ch. priamus Kob. T. perelongata, fere cylindrica, apice breve conico, carneo-albida, læviuscula, nitida; anfr. 12, lentiss.; ap. obliqua, anguste ovata, obsolete et profunde 3-dentata: lam. 1 par., d. I ad basin col., truncaturam simulante, d. 1 marg. minimo, perist. alboincrassato. L. $13 \frac{1}{2}$, d. $4^{3} / 4 \mathrm{~mm}$. (Asia minor, Troas). Ic. f. 1101. (Kob.)

Cli. samius IIts. T. oblongo-conica, leviss. striatula, corneo-fusca; anfr. 7, regulares, ult. basi turgidus; ap. 2-dentata: d. 1 par. compresso sat valido et d. 1 marg. tuberculif., tuberculo ang. nullo, perist. alboincrassato, vix expansiusculo. L. 7, d. $3 \mathrm{~mm}$. (Grocia, ins. Samos). Griech. Moll. 1889 c. f. Ic. f. 1106. - Forma 1) exilis n., t. minor, sursum fortius conica, anfr. $7,1.6^{1} / 2$, d. $2^{1 / 3} \mathrm{~mm}$.

Ch. quadridens Müll. T. ovato-oblonga, ventrosula, superne breviter conica, lævigata, lutescenti-cornea; anfr. 7$7 \% / 2$, regulares, planiusculi, ult. antice albidus, cervice tumidulus; ap. verticalis, subovalis, interdum tuberculo ang., 4-dentata: lam. 1 par., d. 2 colum. (sup. deinde breviss. transversali, re vera basis incrassatus col.), inf. majore, d. 1 marg. conico, perist. labiato, patulo, callo par. fere nullo. L. 9-10, d. $3-3^{1} / 2 \mathrm{~mm}$. (Eur. merid. et media.) Verm. hist. 1774, Ic. f. 308, 723. - Forma 1) minor, 1. 51/2-6, d, 21/2-3 mm.; 2) tridentata W., ap. dente inferiore colum. deficiente (Pyrenææ); 3) pentodon W., ap. lam. acuta secunda parietali prope col. et profundius (Tirolia in Guthal).

Var. prolixus Pini (Atti soc. it. 1879): t. major, cylindraceo-fusif., anfr. 9-11, plani, ap. dentibus validioribus, col. valida, contorta, infra transversa, $d$. infero subbasali, perist. paullo fortius incrassato, marg. colum. magis dilatato; 1 . $12-14$, d. $4-4 \frac{1}{2} \mathrm{~mm}$. (Ital., Gall. merid., Hisp.) Ic. f. 928. Forma 1) attica W., t. gracilior, cylindracea, períst. leviter labiato, breviter expanso, 1. 10-13, d. $31 / 4-31 / 2 \mathrm{~mm}$. (Attica). 
Var. druparumuli Loc. (Ipsa Drap. Conch. 1895): t. perbrevis, ventroso-ovoidea, apice perobtuso, rufocornea, striolata, solida, anfr, 5-6, convexi, regulares, ap. sat ampla, 4dentata; l. 6-9, d. $31 / 4-31 / 2 \mathrm{~mm}$. (Gallia).

Vur. elongatus Requ. (Coq. Corse 1818): t. elongata: conico-turrita, anfr. 91/2, perlente crescentes, ap. obliqua, perist. valde incrassato, plane expanso, lentibus levibus, superiore columellari sæpe evanescente; l. $10-12$, d. $31 / 2 \mathrm{~mm}$. (Corsica).

Var. niso Risso (Hist. eur. mer. 1826): t. ventrosa, fuscocornea, anfr. 6-8, perlenti, ult. cervice gibbo, ap. basi exacte rotundata, recedens, f. 1 par. lamellif., d. 1 marg. parvo conico et d. 1 colum. supra medium producto (raro denticulo besali subinconspicuo), perist. subrecto, labiato, calloso, marg. requaliter arcuatis; $1.6-9$, d. $3-4 \mathrm{~mm}$. (Gallia merid., Ital., Dalm., Bosnia, Herzegowina). Ic. f. 306, 724 .

Var. Innaticus Chr. et $\operatorname{Jan}(\mathrm{Rm}$. Ic. f. 307,724 a. b.): t. elongata, cylindrica, gracilior, anfr. 9-10, perlenti, ap. longior, sinu exteriore angustiore ac peracuto, sinu basali angusto ac obliquo, lam. par. magna, sat retusa, dente in marg. exter. minore, dente colum. basali, exiguo; 1. 8-12, d. 3-4 mm. - Forma 1) callida n., ap. col. callosa, pone basin dentif. producta. (Dalm., Bosnia, Gallia, Alpes-Marit.)

Var. npfelbecki Brancs. (Ver. Trencs. 1888 c. f.), t. brevis et ventricosa, dentibus forte reductis, sæpe obsoletis; l. $9-1 \mathrm{I}$, d. $4 \mathrm{~mm}$. (Bosnia).

Var. Loewi Phil. (Zeitschr. f. Mal. 1844): t. magna, ventrosa, cylindrica. apice conico, obtuso, anfr. 9-10, ap. 4dentata, lam. ang. forti; 1. 15-16, d. $5 \mathrm{~mm}$. (Asia minor).

Ch. planilabris Bttg. T. cylindracea, supra attenuatoconica, fuscocornea; anfr. 7-9, regulares; ap. extrorsum sat forte intorsa, tuberculo et sæe callo par. incrassatis, lam. 1 par. immersa, d. 1 marg. medio intrante, d. 1 colum. subbasali, brevi, gibbosa, adhuc sub sut. in sinulo callo plicif. sæpe forti, perist. late expanso, intus calloso-labiato, extus plano, col. superne oblique stricta. L. $10-11 \frac{1}{2}$, d. $31 / 2 \mathrm{~mm}$. (Thessalia, Euboea in m. Pelion). Jahrb. 1885 c. f. - Forma 1) minor, 1. $7 \frac{1}{2}-9$, d. $3 \mathrm{~mm}$. (Euboea, m. Delphi); 2) major, dent. ap. validis, 1.15 , d. $3 \frac{1}{2} \mathrm{~mm}$. (Græcia, ins. Skiathos).

Var. volensis n.: t. gracilis, cylindracea, supra medium lente attenuata, anfr. 10, lentissimi, ap. callo srepe crasso, extus fortiori, lan. par. valida, marg. colum. edentato, sed col. profunda valida ad basin ap. producta, medio abbreviata $\mathbf{v}$. anguste sinuata; 1. 9, d. 2-21/2 $\mathrm{mm}$. (Volo).

Ch. hippolyti Kob. T. cylindrata v. fere fusif., cornea, nitens; anfr. 9-10, planulati, lenti; ap. 2-dentata, d. 1 par. et 1 marg., callo par. tenuiss. extus tuberculifero, col. profunde, vix conspicue plicata et medjo subincrassata (v. "in- 
flexione mediana obsolete bidentata" Bttg.). L. 101/2, d. 31/4 $\left(2^{3} / 4\right.$ Bttg.) mm. (Euboea, m. Pelion). Jahrb. 1880. Ic. f. 2038. (Kob.)

Ch. thiesseanus (Mss.) W. T. oblongo-cylindracea, apice breviter conico, lævigata, lutescenti-cornea; anfr. 10, planulati, lente regulares, ult. basi compressus; ap. extrorsum vergens, tuberculo ang. forti cum lam. par. valida longissime intrante confluente, dentibus profundis, 1 marg. magno, 2 colum. minoribus approximatis, perist. patulo, alboincrassato, callo par. medio obsoleto. L. $13-14$, d. $31 / 2-3^{5} / 6 \mathrm{~mm}$. (Grocia). Aperȩu Gr. 1879. Ic. f. 2015. - Forma 1) minor, tuberculo ang. sæpius Jibero, callo colum. nullo, l. $11-12 \frac{1}{2}$, d. $22_{15}-3$ $m m . ; 2)$ chilodius $W$., t. major, perist. crasso late expanso, dent. colum. obliteratis, callo par. distinctiore, 1. 17, d. $5 \frac{1}{2} \mathrm{~mm}$. (Euboea ad Kume); 3) heliconicus WV., t. col. dilatato, denticulis 2 tuberculif., l. 15 , d. $31 / 2 \mathrm{~mm}$. (Helicon).

Ch. godetianus Kob. Differt a præcedente præcipue anfr. lentius crescentibus, ult. ad ap. impresso et ad basin fortius compresso, ap. subtriquetra, lam. par. immersa a callo margines conjungente bene separata, d. 2 in col. (supero profundo plicif. desuper intaenti vix conspicuo altero marginali), d. 1 forti in marg. exteriore. L. 13, d. $4 \mathrm{~mm}$. (Graccia, ins. Samos). Jahrb. 1880. Ic. f. 2037. (Kob.)

Ch. denticulatus Pfr. T. cylindraceo-turrita, striatula, oleoso-micans, cornea, sp. elongata, sursum attenuata; anfr. 10, angusti, ult. basi cristatus; ap. semiovalis, obliqua, tuberculo ang. et denticulis 3: 1 par. profundo, 1 ad basin col. arcuatæcallosæ, 1 in marg. exter.; perist. albocallosum, reflexiusculum. L. 7, d. $21 / 3 \mathrm{~mm}$. (Asia minor, Harmanjek). Proc. Lond. 1851. Ic. f. 2030 . (Pfr.)

Ch. scapus (Parr.) Pfr. Præcedenti similis, sed t. carneo-albida, sp. cornea, anfr. 9, plani, ult. basi tuberculatocompressus, ap. rhombeo-ovalis, 3-dentata: d. 1 compresso in par., d. 1 ad basin col. arcuatæ et d. 1 marg. L. 16, d. 51/2, mm. (Asia minor). Mon. Hel. 1853. Ic. f. 2019.

Var, destitutus Mss. (J. Conch. 1873): t. minor, perfecte cylindrica, superne breviter conica, dentibus in col. et in marg. exter. parvulis v. nullis; $1.91 / 3$, d. $4 \mathrm{~mm}$. (Armenia, Turkestan).

Ch. oribatha W. T. subcylindrica, lævigata; anfr. 10 -11, lentissimi, plani, ult. basi rotundatas; ap. semiovatotrigona, 2-dentata (dente par. et d. marg. oppositis, hoc sæpe 
tenue producto), col. elata et concava, usque ad basin ap. producta, ubi interdum dentif.; perist. intus late labiatum, callo par. tenui, extus tuberculifero. L. $9-12$, d. $3-31 / 2$, ap. 1. $21 / 2-3 \mathrm{~mm}$. (Tauria). Binn. Conch. 1887.

Var. discretus W. 1. c.: anfr. 8, convexiuscnli, sut. profunda, ap. margine exter. forte curvato, col. valde callosa, callo par. extus incrassato; $1.74 / 5$ d. $31 / 9 \mathrm{~mm}$.

Var. smyrnensis n.: anfr. 9, convexiusculi, sut. tenui, ap. callo par. incrassato, lam. par. immersa, d. marg. forti, d. colum. marginali intus cum col. elata tenue conjuncta; 1. 10$10 \%$, d. $2 \frac{1}{2} \mathrm{~mm}$. (Smyrna).

Ch. komarowi Bttg. Ch. scapo peraffinis sed differt ap. 2-dentata sc. d. par. compresso, angulum peristomatis sæpe curvatim tangente, altero supramediano in marg. exter, col. planiuscula, parum curvata, omnino edentata, perist. sublabiato, callo par. tenui, L. $12-13$, d. $3^{3 / 4}-4$, ap. $1.3^{1} / 4-3^{1 / 2} \mathrm{~mm}$. (Armenia, Kars). Jahrb. 1880 c. f. Ic. 1996. (Bttg.)

Ch. tricollis Mss. T. forma Pupæ dolii, fuscocornea, pone ap. albescens; anfr. 8, lenti, angusti, ad sut. subangulati, ult. antice sulco tenui longitudinali, ad basin rotundatus; ap. obtuse triangularis, sinulo perangusto, plica ang. verticali, intrante, sepius cum lam. par. continua, d. 1 colum. profundo et d. 1 marg. pervalido obliquo. L. $8-9$, d. $3^{\frac{4}{4}} / 5 \mathrm{~mm}$. (Cancasus). J. Conch. 1876 c. f. Ic. f. 1997.

Var. minor Bttg. (Jalırb. 1880 c. f.): t. minor, magis cylindracea, anfr. 9, lentius crescentes, duo præult. subæquales; 1. $71 / 2-8$, d. $31 / 4-31 / 2 \mathrm{~mm}$. (Armenia, Kars).

Var. excellens Ret. (Ber. Senck. Ges. 1889): t. anfr. ult. fortius impresso, ap. callo par. dilatato utrinque tuberculifero et cum lan. par. conjuncto; $1.7-10$, d. $2 / 5-4 \mathrm{~mm}$. (Batum).

Ch. retrodens Mts. T. ovato-oblonga, fulvo-carnea, sublævigata, nitida, sp. breviter conoidea; anfr. 7, planiusculi, superi lenti, cæteri regulares (vel 2 præult. subæquales), ult. antice planatus, paullo ascendens, basi angustatus; ap. semiovalis, 3-dentata: lam. 1 par., compressa, alta, profunda, d. 1 colum. elongato, basi abrupte truncato, d. 1 marg. tuberculif., profundo, perist. calloso, albo, callo par. tenuiss. L. $9-121 / 2$, d. 4-5 mm. (Dshungaria). Sitz. ber. Berlin 1879. Ic. f. 587. 
Var. Iongulus Cl. (Nachr. Blatt 1894): t. elongatoovata, sat distincte striatula, anfr. lente regulares, ap. dente 1 par. et $d$. 1 parvo inferne in col. crassa; 1.8 , d. $31 / 5 \mathrm{~mm}$.

Var. schmilti Cl. l. c.: t. striatula, fortius versus ap., anfr. celerius crescentes, ap. dente brevi immerso in par., marg. colum. incrassato, magis reflexo; $1.8 \frac{1}{2}, \mathrm{~d} .4 \mathrm{~mm}$.

Ch. aptychus Anc. Præcedenti habitu persimilis, differt præcipue ap. 1-dentata (sc. d. par. retrorsum sito valido ac vix compresso, col. intus curvata, callosa, non tuberculata, ad basin rotundata, perist. expanso, superne stricto, callo par. crasso, extus interdum tuberculifero. L. 10, d. $5 \mathrm{~mm}$. (Turliestan, Wjernoje). Bull. mal. Fr. 1886. (Anc.)

Var. capusianus Anc. 1. c.: t. magis elongata et gracilis, fulva, ap. obsolete triangularis, marg. colum. valde longeque calloso-incrassato; $1.91 \%$, d. $3 \frac{3}{4} \mathrm{~mm}$.

Ch. drymæus W. T. oblonga, superne breve conica, fusca, supra striata, deinde irregul. costata, costis obliquis albidis, in anfr. ult. subverticalibus; anfr. 7, superi sat lenti, medii lati, subæquales, ult. basi rotundatus: ap. 3-dentata: lam. 1 par., profunda, alta, d. 1 colum. mediano, valido, iufra transver'so, d. 1 marg. mediano, crasso, nodif., perist. incrassato, patulo-reflexo, marg. subæqualibus, æque curvatis. L. 9, d. $3 \mathrm{~mm}$. (Turkestan, Aslam-bol)). L. c. 1896.

Ch. retroplicatus (Mts.) W. 'T. cylindrica, apice conico, striatula, albida; anfr. $8 \frac{1}{2}$, planiusculi, omnes lente regulares, ult. major, basi rotundatus, antice sat alte ascendens; ap. semiovata, unidentata, sc. lam. 1 par. compressa, brevi, profunde immersa; perist. calloso, vix paullo patulo, callo par. lato tenui. L. 12, d. $3^{1 / 3} \mathrm{~mm}$. (Turkestan, Wjernoje). Nachr. Blatt 1893.

Ch. leucoptychus (Mts.) Anc. T. ovato-oblonga, cinereo-albida, pliculis albidis confertim sculpta, sp. superne breve conica; anfr. $6 \frac{1}{2}$, superi convexiores, ult. superne vix ascendens, basi attenuatus; ap. semiovalis, subbidentata, sc. lam. par. profunda, valida, col. introrsum calloso-plicata, plica subdentif., elongata; perist. callosum, callo par. simplici. L. $71 / 2$, d. $3^{2} / 3$, ap. 1. $3^{1 / 5} \mathrm{~mm}$. (Turkestan). L. c. 1886. (Anc.)

Ch. entodon Mts. T. oblongo-turrita, striatula, fulvocornea; anfr. 8, sut. sat profunda; ap. truncato-ovalis, par. intus tuberculo crassiusculo munito, perist. incrassato, expanso, marg. exter. superne attenuato, arcuato, colum. lato. L. 12, d. 4, ap. 1. $3^{1} / 2 \mathrm{~mm}$. (Turkestan, Wjernoje). Sitz. ber. Berlin 1882 . 
Var. dextroversus Anc. l. c.: t. major, magis producta, anfr. 9, ult. convexior et dextrorsum excentrice productus, ap. obsolete subtrigonalis, marg. colum. valde declivi, calloso; J. 14, d. medio $4^{2} / 3$, ap. $1.4 \mathrm{~mm}$.

Ch. intumescens Mts. T. cylindrico-oblonga, apice conoideo, striatula, fulvo-cornea, concolor; anfr. 7, lente regulares, ult. ad perist. leviter impressus, ad basin rotundatus, sut. marginata, albida; ap. ovato-rotundata, dente parvo at distincto in medio marg. exter., perist. undique reflexo, albo, valde incrassato, marg. exter. superne angulatim curvato, colum. late reflexo, callo par. tenui extus intumescente. L. $81 / 2$, d. 4, ap. l. $3 \mathrm{~mm}$. (Turkestan, Osch). Moll. Turk. 1874 c. f. Ic. f. 594.

Var. Pedischenkoi Anc. 1. c. (1886): ap. tuberculo prominente, late dentif. intus in marg. exter. et d. minuto marginali in col.; l. \&, d. $31 / 3 \mathrm{~mm}$.

Ch. anomalus 11. T. rimato-perf,, cylindraceo-conoidea, cornea, sub lente dense striata, striis plerisque albidis; anfr. $7-8$, lenti, omnes convexi, ult. superne rectus v. paullo ascendens, ad basin subsaccatus, sut. profunda, marginata; ap. semiovata, 3-dentata: d. 1 par. cum callo sat tenui conjuncto, denticulo 1 in col., dente 1 parvo marginali medio in marg. exter., perist. toto expanso-reflexo et alboincrassato. L. $71 / 2$, d. 2\% mn. (Turkestan, Samarkand). - Forma 1) infans n., ap. omnino edentata. (Pamir). Hanc formam accepi s. n. Ch. intumescentis et typicam at (h. fedtschenkoi.

Ch. haberhaueri (Dohrn) Anc. T. aperte perf., oblongoconoidea, sursum lente attenuata, striatula, griseo-cornea, superne lutescens; anfr. $7-71 / 2$, lente regulares, ult. non ascendens, basi rotundatus, sut. fuscomarginata; ap. subcordif., dentibus 3 subæqualibus validioribus (d. par. lamellif. cum callo par. convexo, d. marg. et colum. suboppositis), perist. infra d. marg. incrassato, reflexiusculo. L. $71 / 2$, d. $21 / 2-3$, ap. 1. $2^{3} / 10 \mathrm{~mm}$. (Turkestan, Turan). L. c. 1886. - Forma 1) curta Anc. l. c., t. minor, minus producta, abbreviata, anfr. $6^{1} / 2$, ap. paullo major, l. $6^{1 / 2}$, d. $2^{3} / 4$, ap. l. $2^{1 / 5} \mathrm{~mm}$.

Ch. secalinus (Mss.) Mts. T. elongata, subcylindrica, sp. sursum attenuata, alba, pellucide griseo-variegata; anfr. 7, ultimi tres subæquales, ult. basi rotundatus, circa riman subinflatus; ap. edentata, perist. vix expansiusculo, incrassato, callo par distincto, extus tuberculifero, marg. exter. superne incurvo, colum. arcuato. L. 9, d. $31 / 2$, ap. $1.3 \mathrm{~mm}$. (Dshungaria). Conch. Mitth. 1890 c. f. Ic. f. 598.

Ch. tardigyrus W. Ch. didymodo similis, sed magis conica, albida, minor quamquam numerus anfractuum major est 
(sc. $71 / 2$ ), anfr. lente regulares, 2 præult. aspectu laterali e dextro multo humiliores et latiores, ap. dente $\mathbf{v}$. denticulis duobus ad basin omnino caret. L. 7, d. $3 \mathrm{~mm}$. (Turkestan, Ssuluku). Ann. Ac. Petersb. 1896.

Ch. torquatus W. T. cylindracea, conico-attenuata, dense peroblique ruguloso-costata, costis concoloribus, extrorsum forte curvatis, cornea, ad ap. late incrassato-lutescens; anfr. $71 / 2$, regulares, ult. postice planulatus, infra lente attenuatus; ap. dente valido plicif. profundo superne in col., marg. verticalibus. L. $11^{1} / 2$, d. $3^{1} / 2 \mathrm{~mm}$. (Turkestan, Taran-basar). L. c. 1896.

(Ch. didymodus Bttg.)

* Sewertzowi a Kob. (Iconogr. 1888).

S. dissimilis Kob. T. aperte umbilicata, mox rimata; anfr. embryonales $2^{1} / 2$ translucidi, læves, ult. circa umbilicum in cristam compressus; ap. ovalis, perist. alboincrassato, expauso. L. $51 / 2-8$, d. 4, ap. $1.3 \mathrm{~mm}$. (Dshungaria). Sitz. ber. Berlin 1882. Ic. f. 580 .

* A m p his c op us W. (Binn. Conch. 1887).

A. ledereri (Zel.) Pfr. T. conico-fusif,, ad sut. distinctius striata, rufocornea, sp. elongato-conica; anfr. $71 / 2$, planulati, ult. antice rectus, basi paullo compressus; ap. semiovalis, 4dentata: lam. 1 par. immersa (et denticulo pr. marg. exter.), pl. 1 colum. et. f. 2 palat. subæqualibus, elongatis, perist. albolabiato. L. 7, d. $3 \mathrm{~mm}$. (Syria, m. Taurus). Mon. Hel. 1868 (Pfr.)

A. michoni (B.) Fag. T. perf,, cylindraceo-oblonga, nitida, sublævis, pallide cornea; anfr 7 , convexi, ult. basi paullo cristatus, albidus, ad ap. contractus; ap. semioblonga, 6-dentata: lam. 2 par. (1. superne, 2. immersa, validiore), pl. 2 colum., pl. 2 marg.; perist. albolabiatum, callo par. nullo. L. 7, d. $2 \frac{1}{2} \mathrm{~mm}$. (Tiberias). W. in Binn. Conch. 1887. (Fagot).

A. sturmi Kstr. T. elongata, cylindrico-turrita, corneoflavida, cervice albida; anfr. $10-11$, angusti, ult. vix $1 / 5$ long. testæ, superne ascendens, basi retrocedens, compressus; ap. 2-dentata: lam. 1 par. longe intrante, interdum tuberculo ang. minimo, d. 1 marg. elongato; perist. expansiusculum, forte albolabiatum, callo par. forti, col. curvata, callosa. L. $71 / 2-$ $91 / 2$, d. 2-21/2 mm. (Asia minor, Brussa). Chemn. Cab. 1845 c. $f$. 
Var. substurmi Ret. (Mal. Blätt. 1886): t. sinistrorsa, subulif., pallida, nitida, anfr. 10 , perangusti, ult. basi rotundatus, antice medio impressus, ap. d. 1 par. et 1 marg., callo valido; $7 \frac{1}{2}$, d. $2 \mathrm{~mm}$. (Tauria).

A. squalinus Rm. T. cylindraceo-turrita, subtiliss. striata, fuscula; anfr. 7, convexi, angusti, regulares, ult. ascendens, basi rotundatus; ap. semiovata, tuberculo ang. dentifero, 5.dentata, dentibus lamellif.: 1 par. valido, curvato, 2 colum. (infero majore), 2 marg. (et sæpius tertio supero minuto); perist. albolabiatum, callo par. nullo. L. $61 / 2$, d. $21 / 2 \mathrm{~mm}$. (Asia minor, Brussa). Zeitsch. f. Mal. 1848. Ic. f. 941.

A. continens Rosen. T. cylindrato-turrita, corneoflavescens, striatula, nitida; anfr. $61 / 2$, perangusti, ult. rotundatus; ap. truncato-ovalis, tuberculo ang. minimo v. nullo, dent. 4 validis: 1 par., 1 colum. alto transverso, 2 marg. (1. infra sinulum, 2. validiore); perist. anguste expansum, leviter labiatum. L. $61 / 4-71 / \mathrm{s}$ d. $21 / 2-25 / 8$ ap. 1. $2 \mathrm{~mm}$. (Transcaspia). Nachr. blatt 1892. (Ros.).

A. ogyptiacus W. T. oblonga, conico-attenuata, striatula, nitida, alba; anfr. 7, regulares, infra ad sut. angulati, ult. superne rectus, basi rotundatus; ap. semiovata, immerse forte albolabiata, lam. tenui ang., 5-dentata: lam. 1 par. forti, 2 colum. immersis (inferiore basali), d. 2 colum. perbrevibus; perist. rectum, acutum, callo par. nullo. L. 10, d. $4 \mathrm{~mm}$. (Aegyptus). Binn. Conch. 1887.

A. eudoxinus Næg. T. umbilicata, conico-fusif., oblique striatula, nitida, lutescenti-cornea, sp. elongata; anfr. $71 / 2$, lenti, convexi, ult. tumidus, basi subangulatus, $1 / 3$ long. teste, sut. immersa, filosa; ap. magna, obliqua, fere circinnata, ad dextrum producta, 5-dentata: d. 1 par. permagno, lamellif., torquato, d. 1 colum., d. 3 marg. (infimo maximo); perist. album, expansum, incrassatum, callo par. extus gibboso. L. 6, d. $21 / 2$ mm. (Asia minor, Tokat). Nachr. Blatt 1894 (Næg.)

\section{B. Ex typo Pupæ.}

T. dextrorsa (rariss. sinistrorsa), sæpius parva, breviter ovata, ovato-conoidea $\nabla$. cylindracea; ap. rotundata $\nabla$. truncatoovata, sæpiss. dentata et parum quam spira brevior, marginibus æqualibus.

\section{Conspectus generum.}

+ T. dextrorsa.

* T. in statu juvcniti plicis postea deficicntibus instructa. 
L a u ria Gray. T. sæpius umbilicata, cylindracea v. cylindrico-conica, obtusa, sæpiss. lævigata, fulvida; anfr. 5-9; ap. lam. et plicis perpaucis longis munita.

0 r c $\mathbf{l} \mathbf{l}$ a Held. T. rimata $\mathbf{v}$. perf, clavata v. cylindrica, apice brevissime conico, striata v. lamelloso-costulata, fusca; anfr. 9-12, angusti; ap. lam. 1 par. et pl. $1-3$ colum.

Cory a W. T. rimato-perf., cylindrica, gracilis, tenuis, costulata (raro lævigata), pallida; anfr. 6-9, planati; ap. oblonga, pauciplicata.

P a g o d i n a Stab. T. matura imperforata (periomphalo forte dilatato), breve cylindrica, lamelloso-costata; anfr. 8-9, convexi, ult. fortiss. ascendens; ap. plicis nullis desuper intuenti conspicuis, sed intus profunde supra umbilicum plica longa et plicula intus in pariete anfr. antepenult.; col. infra clausa, sursum tubulata.

\section{** T. plicis nullis in statu juveniti.}

S a n d a h l i a W. T. umbilicata (umb. circulari profundo, perpendiculari), densiss. capillaceo-striata, forma Orculce, anfr. ult. Pagodine, plicis aperture Torquillce $(2-2-4)$, longis, intrantibus, et plica basali singulari, valida, curvata, immersa.

P u p a Drap. T. cylindrica v. fusif, corneo-flavescens v. rufescens, regul. oblique denseque capillaceo-striata (rariss. lævigato: (3)), anfr. sæpe multi $(10-14)$.

M odicella Ad. T. plus minusve ovato-conica v. turrita, attenuata, brunnea $\nabla$. castanea, sæpe pruinosa, lævigata v. obsolete irregul. striata (raro regul. costulata v. costata); anfr, $5-8$.

Gra n o p u pa Bttg. T. parva, gracilis, attenuato. cylindracea, regul. dense tenueque costulato-striata, cornea; ap. plicis $1-2-4(3)$, remotis, brevibus, angulari nullo, par. compressa, colum. dentif., palat. medio nodiferis, perist. simplici, recto, marg. distantibus.

O d o n t o c y c l u s Schl. T. a basi globosa late breviterque conica, pallide cornea, striata v. costulata; anfr. 6-7, convexi, ult. maximus, ascendens; ap. rotundata, pl. 6-7, lamellif., brevibus, margiualibus. 
P u p illa Leach. T. abbreviato-cylindrica, apice breve conico, lævigata v. pertenue striatula, fusca; anfr. $6-7$, angusti, ult. sæpiss. antice callo transverso; ap. rotundata, edentata v. denticulis perpaucis.

S p li y r a d i u m (Ag.) Ch. T. cylindrica, raro conoidea, obtusa, lævigata; aufr. 6-8, primi lentiss., cæteri regulares, ult. maximus, ventrosus; ap. rotundata, edentata, perist. recto simplici.

I s t h il i a Gray. T. minuta, omnino cylindrica, gracillima, apice summo obtuso, costulata v. striata, albida; anfr. $5-7$, convexi, lenti; ap. rotundata, $2-3$-dentata v. edentata.

I e u c o c h i I u s (Mts.) Bttg. T. cylindraceo-ovata, lente attenuata, alba, opaca, lævis; aufr. 51/2 -7 , convexi; ap. rotundata, forte dentata (lam. par. valida, subbipartita).

\section{it Testa dextrorsa vel sinistrorsa.}

V e r t i g o Müll. T. minuta, obeso-ovata, plus minusve ventrosa, lævis v. striatula, fusca; anfr. $41 / 2-6$, ult. magnus, rotundatus; ap. ovata, dentata (rariss. edentata).

\section{Gen. IL is I r i a Gray (Turton Man. 1840).}

Conspeetus.

† Ap. lam. et 1,l. 1-2 (par. 1-lamellato).

it Ap. lam. et pl. 4-5 (par. 2-lamell.). (2.)

tif Ap. lam. et den. multis armata. (3.)

\section{(1.)}

L. cylindracea Da C. T. umbilicata, ovato-cylindracea, nitidula, fere lævis; anfr. 6-7, ult. basi compressus; ap. oblique subtriangularis (marg. exter. curvata, colum. subrecto), lam. par. longa superne incrassata et marg. exter. affixa, pl. colum. obsoleta, perist. late expansum, albolimbatum. L. 4, d. $2 \mathrm{~mm}$. (Europa in terris ad oras marium, nec non in Tirol. et Helv., (aucas., Asia min., Armenia). Test. Brit. 1798 c. f. Ic. f. 327. - Forma 1) incrmis W. (F. eur. 1876), ap. omnino edentata (Suecia, Visby);2) eurtu (V. l. c., t. ovoidea, tumida, anfr. 6 61/24 1. 3, a. $2 \mathrm{~mm}$; 3) edentula II. T., ap. dente colum. nullo.

Var. misella Paul. (F. Sard. 1882 c. f.): t. minor, fragillima, ap. minor, rotundata, edentata v. denticulo obsoleto par., perist. parum dilatatum; 1. 3, d. $1^{3 /} / 4 \mathrm{~mm}$. (Sardinia). 
Var. subperforata B. (Mal. Alg. 1864): t. perf., anfr. ult. basi subrotundus. (Alg., Constantine).

Var. ancomostoma (Lowe) Pfr. (Mon. Hel. 1848): t. cylindracea, anfr. 6, convexi, perlenti, lam. par. tenuis, sut. parallela, superne obsolete incrassata, e margine separata, perist. limbo angusto subconcolori; 1. $3 \frac{1}{2}-4$, d. $2 \mathrm{~mm}$. (Ital. bor., Triest, Hisp. prov. Galicia, Gr. ad Varvara, Suec. ad Visby).

Var. gracilis Iss. (Moll. Pisa 1866): t. magis producta, gracilior, superne minus diminuta; $1.31 / 2, \mathrm{~d} .1 \frac{1}{2} \mathrm{~mm}$. (Ital. bor.).

Var. montigena W. (Exk. F. 1884): t. minor, nigrescens, tumida, sp. brevi, rotunlata, col. plica emersa et intus dente conico instructa; 1. 3, d. $2 \mathrm{~mm}$. (Suec. in monte Skäralid).

Jur. inaequalis W. (Binn. Conch. 1887): t. ovatocylindracea, rufescens, anfr. 6, convexi, antepenult. multo (fere duplo) humilior quam penult., ult. tumidus, lam. par. vix incrassata, a marg. separata, dente adposito, pl. colum. longe emersa, in medio columellre posita, perist. anguste limbatum, rufescens; 1. 3\% $/ 2$ d. $2 \mathrm{~mm}$. (Græcia).

Var. villae (Ch.) Kstr. (Mon. 1845 c. f.): t. fere cylindrica, subtiliter striata, anfr. convexiusculi, ult. non compressus, ap. subovata, perist. peranguste expansum, albido-corneum; 1. $4^{1} / 2$, d. $1^{1} / 2 \mathrm{~mm}$. (Helv. ad Brienz).

Var. wmbilicus Roth (Diss. 1839 c. f.): t. perspective cylindrico-umbilicata, ovato-cylindrica, striatula, sp. sursum lente attenuata, anfr. ult. periomphalo lato, ad basin acute cristato-compressus, ap. subtriangularis, lam. par. longa, tenuis, conjuncta, perist. albocallosum; l. 3, d. $1^{3} / 4 \mathrm{~mm}$. (Græc. ins. Syra, Dalm. merid., Albania).

L. fugoti n. sp. T. semiobtecte perf., obesa, subovata, perobtusa, cornea, levis, uitida; anfr. 5, superi lente regulares, convexi, sut. profunda, ult. duplo major, convexus, antice horizontalis; ap. semiovalis, marg. exter. regul. arcuato, colum. subverticali, superne dilatato at reflexo, lam. 1 par. tenui longiss. et dente 1 acuto in columella. L. $1^{3 / 4}$, d. $1^{1 / 4} \mathrm{~mm}$. (Hisp., Sarria pr. Gerona: Coll. Cleveana in Upsala).

L. poupillieri B. T. perf., umbilico arcuatim dilatato, abbreviato-cylindrica, tenuis, obtusa, nitidiss., fere lævis; anfr. 7, ult. postice ventrosus, basi compressus, superne valde ascendeus; ap. semiovata, lam. par. longa, teuui et $d$. angulari, perist. plano, albolabiato. L. 4, d. $2 \mathrm{~mm}$. (Algeria). Mal. Alg. 1864 c. f. (B.)

L. sempronii Ch. T. perf.-umbil., cylindracea, obtusa, lævigata $\nabla$. substriata, corneo-flava, nitidiss.; anfr, 5-6, subconvexi, ult. sub. ventrosus, basi subrotundatus; ap. semiovata, lam. par. subobsoleta, teuui, libera, pl. colum. nulla, 
perist. peranguste expanso, tenue corneolabiato. L. 3, d. $11 / 2$ mm. (Gall. merid., Helv., Tïrolia, Ital., Sard., Morea, Caueasus). Cat. moll. Sec. 1837 c. f. - Forma 1) ditucida Z. ap. Rm. (Ic. f. 326), ap. omnino inarmata $v$. dente par. rudimentali, $1.2-3, d .1-1 \frac{1}{2} \mathrm{~mm}$. (Gall. merid., Tirol., Sard., Græcia).

Far. sabaudina Loc. (Conch. Fr. 1894): t. tenuis, tenue et densiss. striata, nitida, anfr. 7, ap. lam. par. arl insertionem marg. exter. porrecta et plica parva in medio col., perist. interrupto, expanso, reflexo, incrassato; $1.31 / 2-4$, d. $1^{3} / 4 \mathrm{~mm}$. (Gall. ad Chambery).

Var. caspia Pfr. (Mal. Blätt. 1871): t. gracilior, fere oblonga, anfr. arctius volutis, minus convexis, ult. et ap. minus altis, lam. par. longiss., validiore, perist. sub sinulo subincrassato; 1. 3, d. 1 $1 \frac{1}{3}-1^{2} / 2 \mathrm{~mm}$. (Caucas.)

I. Vulcanica Kstr. T. aperte perf., ovata, lævigata, tenuis, sericea; aufr. 7, plauiusculi, ult. $2 / 5$ long. testr; ap. magna, ovalis, dente minuto par., perist. acuto. L. 3, d. $11 / 2$ mm. (Sard. ad Nurri et Taconi). Mon. 1815 c. f. (Kistr.)

\section{(2.)}

I. anglica Fér. T. ovato-cylindracea, ventricosa, subtiliter striatula v. lævigata, rufocolnea; anfr. $6-7$, planulati; ap. lam. et pl. 5: lam. 1 ang. longa, pervalida, flexuosa, extus callo conjuncta, lam. I par. immersa, pl. 2 colum. (sup. longa, emersa, inf. minutiss.), pl. 1 palat., subbasali, longiss., marg. exter. calloso, superne sinuato et dentif. incrassato. L. $3^{\mathbf{1}} / \mathbf{2}$ $-3^{3} / 4$, d. $2 \mathrm{~mm}$. (Britan., Gallia, Tusit., Alg.). Prodr. 1821. Forma 1) globoso-conica W. (F. eur. 1876), t. parva, anfr. 6, 1. $2 \frac{3}{4}$ -3 , d. $2 \mathrm{~mm}$.; 2) cylindracea W. 1. c.. t. major, 1. 6, d. $2 \mathrm{~mm}$.; 3) castanea W. l. c., t. basi pallidior, perist. carneo.

Var. gunhildae W. (Binn. Conch. 1887): perist. tenue labiatum, lam. angulari a margine bene disjuncta. (Brit. ad Scarborough).

Vur. numidica B. (Mal. Alg. 1864 c. f.): t. eleganter striata, lam. ang. valida superne libera, lam. par. infera deest; 1. $3 \%$, d. $2 \mathrm{~mm}$. (Algeria).

L. pulchra Ret. T. ovato-oblonga, dense costulata, pallide cornea; anfr. $7-8$, ult. ascendens, basi rotundatus; ap. lam. et pl. 5: lam. 2 par. (exteriore margini callo dilatato adnexo), pl. 2 colum. (iuf. validiore), lam. 1 basali, longa, intus non elevata, dente 1 oblongo in medio marg. exter., nec non denticulis 5 colum. externe; perist. callosum, planum. L. $3 \frac{1}{2}-4$, d. $2 \mathrm{~mm}$. (Circassia; Taurus). Mal. Blätt. 1883. 
Var, bilabintu Ret. (Mal. Blätt. 1886): t. dense costulato-striata, anfr. ult. medio longitudinaliter impressus, superne tumidus, lam. par. exterior superne callo tenui marg. colum. et callo fortiore (sed non dilatato) marg. exter. adnexa, denticulis colum. nullis, perist. iluplicatum. (Tauria).

Var. nitens Bttg. (Nachr. blatt 1888): t. major, castanea, unicolor, nitens, anfr. angustius costulato-striati, perist. duplex, labio interno aurantiaco; 1. 4-41/2, d. $2 \frac{1}{4}-2 \frac{1}{2} \mathrm{~mm}$. (Caucasus).

L. pontica Ret. T. ovata, dense costulata, pallide cornea; anfr. 7, ult. ascendens, medio deplanatus, basi compressus, subtus carinatus; ap. lam. et pl. 5: 2 par. (media parva, profunda), l colum. longa, obliqua, 1 basali, brevi, profunda, pl. 1 palat. longa, dente cristif. $v$. duplici in medio marg. exter. et denticulo ang.; perist. albolabiatum. L. $3^{1} / 2$, d. $2^{1} / 5$ mm. (Asia minor ad Samsun). Ber. Senck. Ges. 1889.

L. superstructa Mss. T. cylindrica, striata, sp. ovatoconica; anfr. 8 , ult. valde ascendens; ap. lam. et pl. 5 (2 par., exteriore valida, antice duplicata, 2 colum., infer. magna, emersa, l subbasali, intus elevata) et dente l cristif., marginali, supra medium; perist. late expansum, marg. exter. superne profunde excisum, postea duplicatum, valde callosum, aurantiacum, crenulatum. L. 5-6, d. $2 \frac{1}{2}-2^{3 / 4} \mathrm{~mm}$. (C'aucasus). J. Conch. 1876. - Forma 1) minor 1. $41 / 4$, d. $21 / 4 \mathrm{~mm}$.

Var. Iederi Bttg. (Jahrb. 1883 c. f.): perist. simplex, albidum, lam. par. exterior antice simplex, denticulo adjacente, dens marg. intus non productus; $1.5^{1} / 4, d^{2} \cdot 2^{1 / 2} \mathrm{~mm}$. (Imeritia).

Var. unibasalis Bttg. (Jahrb. 1886 c. f.): ap. lam. par. majore non tuberculifera et pl. palatali unica (nec pl. pal. supera minore nec pl. basali); 1. 5, d. $2^{3} / 5 \mathrm{~mm}$. (Suanetia).

L. zonata Bttg. Præcedenti similis, sed differt t. minore, ad basin distincte fusco-unizonata, ap. extus sub sinulo forte foveif. impressa et dente marginali lamellæ instar in ap. intrante. L. $3^{1} / 2-4$, d. $2-2^{1} / 8 \mathrm{~mm}$. (Caucasus). Jahrb. 1883 c. f.

I. caucasica (Parr.) Pfr. T. oblongo-ovata, lævigata; anfr. $81 / 2$, ult. basi compressus; ap. lam. 2 par. (interiore profunda, dentif., exter. longa), pl. 1 basali longa, denticulo 1 et pl. 1 valida, lata, profunde sita in col,; perist. breviter dilatatum, vix labiatum. L. 6, d. $3 \mathrm{~mm}$. (Caucasus). Mal. Blätt. 1857. (Pfr.)

Gen. O r c u I a Held (Isis 1837).

Conspectus.

† T. porf. v. umbilicata, cylindrico-conica, lente sursum attenuata. (1). 
t† $T$. rimata, cylindrica, apice breve conico $v$. rotunclato.

*) Ap. marg. distantibus v. temuiss. conjunctis, plica colum. brevi, profunda. (2).

**) Ap. marg. continuis, pl. colum. longa, emersa. (3).

0. dolium Drap. T. oblongo-cylindracea, apice conica, tenue striata; anfr. 9-10, angusti; ap. verticalis, semiovata, palato antice albocalloso, lam. 1 par. obliqua, emersa, intus longiss., pl. 2 colum, horizontalibus, non marginalibus. Is. 7, d. $2 \frac{1}{2}-3 \mathrm{~mm}$. (Al Aragonic Hispanice ad Hungariam). Tabl. 1801. Ic. f. $330-31$. - Forma 1) major, 1. $9-10$, d. $3 \frac{1}{2}-3^{3 / 4}$ mm. (Hung.); 2 obesa IV., l. 6-8, d. 4 mm. 3) gracilis Haz., l. 8, d. $21 / 3$ mm.; 4) minor, 1. $6^{2 / 3}-6^{1} / 2$, d. $2^{3 / 4}-3 \mathrm{~mm}$; 5) tumida Haz., 1. 5-6, (l. $2 \frac{1}{3}-3 \mathrm{~mm} . ; 6$ ) iniplicata P. \& M., ap. pl. colum. supera obsoleta; 7) plagiostoma Al. Br., t. parva $\left(6-7: 2{ }^{\prime} / 2-3 \mathrm{~mm}\right.$.), pl. colum. obsoletis, profundis; 8) par IV., ap. pl. colum. validis, longe et aqualiter emersis; 9) quadriplicata Loc. (Var. mal. 1881), ap. pl. colum. 3 (Gall. al Lyon, Galic. in m. Tatra); 10) cebrutica W. (Binn. Conch. 1887), ap. lam. par. 2 prope jacentibus (m. Cebrat); 11) implicate (Brs.) Cl., ap. pl. colum. obsoletis v. nullis (Hung.; cfr. plagiost.); 12) triplicute (Brs.) Cl.; t. major et latior, pl. colum. 3, l. 9, d. $2 \frac{1}{2}-3 \mathrm{~mm}$. (Hung.; cfr. quadripl.); 13) arcegonicre n., t. tenuiss. striatula, lutescenti-cornea, ap. callo palatali angusto translucente, tuberculo ang. et lam. par. tenui, brevi v. brevissima, cæterum edentata (Hisp., Aragonia).

Var. brancsiki Cl. (F. Oest.-Ung. 1887 c. f.): t. elongata, angusta, cylindrica, a medio sursum sensim attenuata, ap. pl. colum. supera semper deficiente; forma 1) elongata, anfr. $10 \frac{1}{2}-11,1.8$, d. $3(8: 2 \%, 10: 21 / 2) \mathrm{mm}$; 2) curta, brevior, anfr. $9-9^{1 / 2}$, convexioribus, 1. 7, d. $2^{4 / 5}\left(7: 2^{1 / 2}, 6: 2^{1 / 2}\right) \mathrm{mm}$.

0. transversalis W. Præcedenti peraffinis, sed differt t. umbilicato-perf., obesa, cylindrica, anfr. tantum tribus superioribus conum breviss. formantibus, anfr. lentiss. crescentibus, transversalibus, sut. ubique horizontali (exc. ad anfr. ult. ubi forte ascendente), ap. parva, lam. et plicis omnibus emersis, perist. recto, acuto, simplici, callo par. lato. L. $6^{3} / 4$, d. $2 \frac{1}{2} \mathrm{~mm}$. (Grceia in $m$. Pindus ad T'schumerka). Nachr. blatt 1893 .

0. gularis $\mathrm{Rm}$. T. oblongo-cyliudracea, apice obtuse conico, lævigata; anfr. 9, lenti, ult. antice tumido-convexus, fulvidus; ap. verticalis, semiovalis, lam. par. 1, valida, pl. colum. 1, subretusa (interdum denticulo superne prof. in col.), palato antice callo albo medio introrsum plicifero, perist. tenui, corneo. L. $61 / 2-71 / 2$, d. 3 min. (Tirolia merid., Carinth., Carn., Tatra). Ic. f. 333. - Forma 1) spoliata Rm. (Ic. f. 334), t. cervice tumidiore, callo palatali erassiore, pl. palatali nulla; 2) restituta W. (Binn. Conch. 1887), ap. pl. 2 colum. fortibus, longis, usque ad marginem parallelis (Carn. ad Stein). 
0. conica Rm. T, ovato-conica (a basi lata subtransversali conico-acuminata), striatula; anfr. forte convexi, ult. basi non superue forte dilatatus; ap. obliqua, semiovato-subtriangularis, palato non incrassato, lam. 1 par. longa, pl. 2 colum. horizontalibus, non emersis. L. $6-7$, d. $3-3^{1} / 2 \mathrm{~mm}$. (Styria, Illyria, Croat. merid.). Ie. f. 332.

0. schmidti Kstr. T. umbilico parvo rotundo, brevis et obesa, subcylindrica, superue in conum brevem exiens, tota tenue membranaceo-costulata; anfr. convexi, ult. ne minime tumidus; ap. verticalis, rotundata, palato albido non incrassato, lam. 1 par. sat valida, pl. colum. 2 altis, brevibus, horizontalibus, emersis. L. 5, d. $2 \mathrm{~mm}$. (Montenegro). Mon. 1845 c. f.

0. jetschini Kim. T. perf., ventrosulo-cylindracea, apice perbrevi et late conico, meml,ranaceo-costata; aufr. angusti, ult. non tnmidus; ap. subverticalis, semiovata, palato antice albo-incrassatulo, lam. 1 par. longa, pl, 2 colum. horizontalibus, fortibus et emersis. L. $6^{1} / 2-7$, d. $3^{1} / 2-3^{2} / 3 \mathrm{~mm}$. (Transsylv., Banatus). Beitr. Moll. Siebenb. 1883.

0. doliolum Brug. T. obovato-cylindracea (sursum paullo latior), apice rotundato, tenue lamelloso-costulata (juv. ad sut. spinulosa); anfr. 9, ad sut. infra subangulati, ult. valde ascendens; ap. rotundato-semiovata, sæpiss. denticulo ang., lam. 1 par. longe emersa et immersa, compressa, pl. colum. 1-2 profundis (una obsoleta). L. $5-6$, d. $2^{1} /{ }_{3}-2^{1} / 2$ nım. (Eur. media \& merid., Caucas., Syria, As, minor, Armenia, Persia). Euc. meth. 1792. Ic. f. 328-329. - Forma 1) curta W., t. obtuse subovalis, aretispira, 1. $4-4^{3} / 4$, d. $2 \mathrm{~mm}$; 2) critica (Zel.) Pfr. (MIal. Bl. 1856), ap. lam. par. tenuiss., pl. colum. desunt (lns. Syra, Gall. ad Lyon, Tauria); 3) major Loc., 1. 6 $\frac{1}{2}-7$, d. $3-3^{1 / 4} \mathrm{~mm}$. (Gall., Sicil.)

Var. intermedin Ret. (Mal. Bl. 1882 : anfr. 9, perist. conjunctum, pl. colum. 3 (media brevis); 1 . $6,2.2 \frac{2}{3} \mathrm{~mm}$. (Tauria).

Var. Latumensis Ret. (Ber. Senck, Ges. 1889): t. callo cervicis forti et callo par. valido, vel major $\left(1_{1} 6^{1}, 2-9, d^{2}, 2^{1} / 2-3\right.$ mm.), subcostulata, pl. colum. distinctis, vel minor (1. 5-7, d. $2 \mathrm{~mm}$.), sublævigata, pl. colum. profundissimis. (As. minor ad Batum).

Var. tereticollis W. (Ann. Ac. St. Pet. 1896): t. brevis et obesa, fortius et distantius lamelloso-costulata, anfr. \&, perangusti, ult. postice perangustus (penultimo non altior), teres (nec lente oblique declivis), lam. 1 par. longa, pl. 1 colum. tenui sat producta, d. ang. nullo, perist. late patulo; 1.4 , d. $1^{2} / 3 \mathrm{~mm}$. (Persia ad Siaret). 
Var. alvinu (B.) Loc. (Conch. Fr. 1894): t. fere cylindrica, anguste elongata, forte striata, anfr. 10, ap. anguste ovalis, lam. 1 par., pl. colum. 2, immersis, perist. expanso, reflexo; 1. 51/2-6, d. $2^{1 / 4} \mathrm{~mm}$. (Gall., Alp.-Mar.). Loc.

Vur. sulblaevis (B.) Loc. 1. c.: t. subcyliudrica, plus minusve obsolete striata, anfr. 8, ap. obovali-rotundata, lam. 1 par. magna at tenui, pl. 1 colum. parvula, perist. expanso, reflexo; 1. 51/2-6, d. $2 \mathrm{~mm}$. (Gall. ad Menton). Loc.

Var. saint-sinuonis B. ap. (Gourd., B. S. Toul. 1881), t. subcylindrica, infra attenuata, distanter lamelloso-striata, cinerascens, anfr. 10, ap. lam. 1 par. magna, tenui, pl. 2 colum. profundis, infer. majore, perist. reflexo; 1. 51/2-6, d. $2^{1 / 2} \mathrm{~mm}$. (Gall. merid.).

Var. museei (B.) Loc. l. c.: t. subcylindrica, elongata, infra attenuata, dense tenueque striata, anfr. 10, ap. parva, rotundata, ad basin paullo retusa, lam. 1 par. sat forti, pl. 1 colum. profunda, robusta, perist. reflexo; $1.5^{1 / 2}-6^{1 / 2}, \mathrm{~d} .2 \mathrm{~mm}$. (Gall. merid.). Loc.

$V a r$. bourguignati (Macé) Loc. 1. c.: t. subcylinulrica, brevis et obesa, tenue distanter striata, anfr. 8, ap. obovalis, basi rotundata, lam. 1 par. forti et valde curvata, pl. 2 colum. immersis (infer. majore), perist. expanso, reflexo; $1.5, \mathrm{~d} .21 / 2 \mathrm{~mm}$. (Gall., Alp.-Marit.). Loc.

Var. macrotriodion (B.) Loc. I. c.: t. eylindrata, perbrevis et perobesa, tenue denseque striata, anfr. 8, ap. subrotundata, lam. 1 par. forti et forte curvata, pl. 2 colum. immersis (infer. magna et curvata), perist. expanso, reflexo; l. $4^{3} / 4$, d. $2^{3 / 4} \mathrm{~mm}$. (Gall., Alp.-Mar.). Loc.

0. scyphus (Friw.) Pfr. T. cylindrica, apice breviss. conico, dense striatula; anfr. $10-11$, convexi, ad sut. rotundati, ult. multo minus ascendens; ap. semiovata, denticulo ang., lan. 1 par. et pl. 2 colum. profundis, brevibus. L. $8 \%-9$, d. $21 / 2-3 \mathrm{~mm}$. (Grecia cum insulis, Asia min., Syria). Zeitschr. f. Mal. 1848.

Var. turcica Let. (Ann. mal. 1870): anfr. 9, convexiusculi, ult. breviter prorsus ascendens, lam. par. valiela, pl. colum. desunt; 1. $6 \frac{1}{2}$, d. $2 \% \mathrm{~mm}$. (I. Santorin et Rhodus, Smyrna, Alexandria .

0. sirianocorensis Mss. T. fere cylindrica, apice convexo-conico, acuto, albido-cornea; anfr. 10, superi 5 tenue costulato-striati, cæteri planulati, striatuli, ult. parum ascendens, basi subcompressus; ap. ut doliolum, sed pl. colum. etiam oblique intuenti vix conspicuis, perist. undique expanso, marg. exter. supra tenui, deinde rapide incrassato. L. 9 , d. $4^{1 / 2} \mathrm{~mm}$. (C'ypria). Coq. Bell, 185̃̃. Ic. Suppl. 1. t. 14 f. 9. (Kob.) 
Var. orientulis (Parr.) Pfr. (Mal. Blätt. 1861 c. f.): t. major, col. superne biplicata, perist. tenui, marg. exter. vix expanso, colum. dilatato, patente; 1. 12, d. $5 \mathrm{~mm}$. (Syria, l'alæstina). - Forma 1) mitida Mss. (J. Conch. 1874), t. recte cylindrica, vix striatula, nitida, ap. late semiovata, pl. 2 colum. distinctis, obliquis.

Var. obess Blanck. (Nachr. blatt 1889): t. magis ovata quam clavata, marg. colum. obliquiore; 1. 11, d. $5 \mathrm{~mm}$. (Syria ad Bab el Haua).

Var. cedrorau W. (Binn. Conch. Suppl. 1890): t. cylindrica, apice rotundato, anfr. subplani, arcti, ad sut. paullo angulati, superi leviter striatuli, cæteri laves, ult. basi rotundatus, superne subhorizontalis, ap. subsemirotundata, lam. par. longa, pl. 2 colum. breviss. conspicuis; $1.11, d .4 \mathrm{~mm}$. (Libanon).

0. Hesopotamica Mss. T. cylindracea, apice brevi obtuse conico, pallida v. cornea; anfr. 9, superi sat convexi, valde membranaceo-costati, cæeteri planulati, lævigata, ult. basi rotundatus; ap. lam. 1 par. remota et pl. 2 colum. brevibus, perist. expanso, ntarg. lamina callo, ad insertiones iucrassato, junctis, exter. regulari, medio non iucrassato. I. $7-9$, d. $3-3 / 4 \mathrm{~mm}$. (Mesopotamia). J. Conch. 1874.

0. raymondi B. T. cylindrica, perobtusa, eleganter dense striatula et costulis nembranaceis elevatis perdistantibus, in anfr. supra medium seto longo instructis, ornata; anfr. $8-9$, convexiusculi, ult. basi rotundatus, superne ascendens; ap. oblonga, lam. I par. longa, recta, pl. 3 colum. tenuibus, longis, emersis (media fortiore). L. 3/4 $-4^{1 / 2}$, d. 2-21/4 mm. (Syria, Caucasus). Moll. nouv. \&c 1863 c. f. - Forma 1) minor (typus ap. B.), $1.2 \frac{1}{2}$, d. $1 \frac{1}{2} \mathrm{~mm}$., anfr. 7 .

Var. trifilaris Mss. (Coq. Schl. 1863): t. apice proluctiore, connm breviss. formante, costulis distantioribus, majoribus, anfr. infra ad sut. et nlt. ad basin distincte angulatis, pl. colum. fortioribus et longioribus; $1.41 / 2-5$, d. $2-2^{3} ;$ $\mathrm{mm}$. (Cancasus).

Var. bifilaris Mss. (J. Conch. 1873 c. f.): t. inverse cylindrato conica, superne latior quam medio et infra, apice plano, anfr. superioribus convexioribus, media parte fere angulatis, pl. colum. 2, inferiure fortiore; l. $4^{1} / 8-4^{1} / \mathrm{s}$, d. $2-2^{1} / 2 \mathrm{~nm}$. (Armenia ross., Abchasia). - Forma 1) longior Ret. (Ber. Senck. Ges. 1889 , t. major, apice productiore, ap. majore, col. fortius contorta, 1. $4 \%, 10-5 \%$, d. $2-2 \%$ mm. (Samsun); 2) intermediu Ret. l. c., ap. pl. media colum. pertenui v. nullo (Samsun). 
Gen. C o r y II a W. (Binn. Couch. 1887).

Couspectus.

† Ap. plicis palat. nullis.

t† Ap. plicis palat. instructa.

*) T. costulata.

a - T. cylindrica v. supra medtium subclavata. (2.)

$b$ - T. siqura medium regual. attenuata. (3.)

**) T. levis v. lavigate.

a - Plica palut. profunde immersa; callus par. validus.

a) Ap. perangusta, infra latior.

B) Ap. angusta, infore quam superne angustior. (5.)

l-Plice palut. parum immersa; callus par.fere nutllus. (6.)

C. truncatella (Z.) Pfr. 'T. albida, tenuis, costata, costis verticalibus rectis; aufr. $6-7$, convexiusculi, ult. penultimo longior, basi rotuudatus; ap. semiovalis, edentata, marg. late distantibus, callo tenui junctis, exterior'e medio intus incrassato. I. $3^{1} / 2-4$, d. $1^{3} / 4-2 \mathrm{~mm}$. (Carn., Carinth., Istria, Croat. merid., Dalm.). Symb. 1846. Ic. f. 733.

Var. Formosa (Parr.) Kstr. (Mon. 1845 c. f.): t. major $\left(5 \frac{1}{2}: 2 \mathrm{~mm}\right.$.), costulata, anfr. 8 , perist. margine exter. fortius impresso. (Dalm.)

Var. biarmata Bttg. (Ber. Offenb. Ver. 1S50): t. gracilior, distantius costulata, ap. pl. par. distincta et denticulo magno calloso marg. exter., callo par. denticulato; $1.35 / \mathrm{s}$, d. $11 / 2 \mathrm{~mm}$. (Dalm.)

C. parreyssi (Friv.) Pfr. T. albida, tenuis, densiss. costulato-striata, costulis verticalibus paullo curvatis; anfr. $7-7^{1} / 2$, planulati, ult. penultimo longior, ult. basi angustatis; ap. oblonga, marg. late distantibus, callo forti junctis, lam. par. $1-3$, tenuibus, pl. colum. 1. L. $5 \frac{2 / 3}{3}$ d. $1 \frac{2 / 3}{\mathrm{~mm}}$ (Turcia, Banatus, Transsylv.). Mon. Hel. 1848.

Vur. caesa W. (F. eur. 1876): t. gracilis, anfr. 2 ultimi aequales, ap. margine exter. superne incisura profundiuscula munita; l. 51/2, d. $1 \frac{1}{2}-1^{2} / 3$ mm. (Transsylv., Banatus).

Var. aruntu Cl. (F. Oest.-Ung. 1887): ap. dente lamellif. in medio et plicula in utraque extremitate parietis, col. interdum denticulo et margo exter. superne tuberculo armatis. (Transsylv.) 
C. Iamellata $\mathrm{Cl}$. T. cylindrica, apice rotundato, alba, nitida, regul. striata, striis perobliquis, argutis, densis; anfr. 7, ult. basi calloso-compressus; ap. angusta, alta, lam. 1 par., emersa et longe intrante et lam. dentif. in angulo colum., col. profundissime in plica compressi, medio dente triangulari forti, denticulo remoto basali, nec non dente forti marginali, extus fovea profunda correspondente. L. 41/2, d. $1^{3} / 5 \mathrm{~mm}$. (Transsylv.). F. Oest.-Ung. 1887. (Kim.)

C. ferrari Porro. T. infundibulif. umbilicata, cylindricoclavata, dense costulato-striata, sericea, corneo-brumnea; anfr. 7, ult. penultimo fere duplo altior, infra elongata, carina unbilicali arcuata; ap. angusta, elongato-subtriangularis, lam. 1 par. et pl. 1 colum. validis, pl. 2 sat fortibus, profundis, et tuberculo basali in palato, tuberculo valido supra mediun in marg. exteriore, callo par. valido. L. 5, d. $3 \mathrm{~mm}$. (Ttalia super., Tirolia). Mal. Comasca 1838 c. f., Poll. Bull. soc. Ital. 1887 c. f. Forma 1) elongata Porro 1. c., t. gracilis, anfr. 8, 1. 41/2, d. 11/2 mm.; 2) guttula Porro l. c., t. obesa, cylindraceo-ovata, anfr. ult. infra fortius angustatus, ]. 4, d. $2 \mathrm{~mm}$.

Var. curts Loc. (Conch. Fr. 1894): t. subcylindrica, perbrevis, perobesa, basin versus vix attenuata, anfr. $7-8$, sat convexi; ap. trapetsoidea, basi paullo retusa, superue subcanaliculata, lam. 1 par. magna, emersa, pl. 1 colum. magna, immersa, pl. 3 palat. (1. tuberculif. in perist., cæteris rudiment., vix conspicuis); l. $3^{2} / 4-4$, d. $1^{3 / 4} \mathrm{~mm}$. (Gall., Alp.-Marit.)

C. bielzi $\mathrm{Rm}$. T. rimato-perf., cylindracea, apice rotundato, subtiliss. striato-costulata, corneo-lutescens; anfr. 9, ult. basi leviter compressus, supra ascendens; ap. oblongo-semiovata, lam. 1 par. valida, pl. 1 colum., pl. 3 palat. (media elongata), perist. brunneo, marg. distantibus callo par. tenui. L. 51/2-6, d. 2-21/3 mm. (Transsylv., Hungaria, Galicia, Moravia). Ic. f. 942 .

Var. euodon IV. (Binn. Conch. 1887): ap. lam. 2 par. (exteriore parva, tenui, profunda), pl. 2 colum. (1. longa, forti, 2. dentif.), $\mathrm{pl}$. 3 palat. (3. verrucif.), jalato sæpius albidocalloso; 1. 5-7, d. $2 \mathrm{~mm}$. (Galicia, Transsylv.)

Var. romипiс⿰丿 Kim. (Beitr. Moll. Siebenb. 1890): t. striis malto tenuioribus et obsoletioribus sculpta, lam. par. rudimentali, pl. colum. superne posita et obsoleta, pl. palat. 2 superis levibus, infima deficiente; $1.4^{3} / 5, \mathrm{~d}^{3 / 3} / 5 \mathrm{~mm}$. (Transsylv.)

C. blanci B. T. late infundibulif. umlilicata, cylindraceoconoidea, capillaceo-costulato-striata; anfr. 9, lente regulares, ult. compresso-angustatus, carina umbilicali acuta; ap. sinuosa, 
superne angulata, lam. 1 par. profunde, valida, pl. 1 colum. superna, immersa, pl. 2 palat. profundis (1. punctif.) et dente conico in marg. exter., perist. continuo, soluto. L. 5, d. 2 mm. (Galli, Alp.-Marit.). Soc. Lannes 1873.

Yar. niciensis Poll. 1887 c. f. 1. c.: ap. pl. palat. 4, margine colum. calloso, leviter dentato. (Nizza).

C. valsabina Spiu. T. subperf., cylindrica, lrevigata, nitida, corneo-fulvida; anfr. $9-10$, ult. compressus, carina umbilicali medio angulata; ap. subrimaf., supra canaliculata, infra rotundata, lam. 2 par. (exteriore brevi, profunda), pl. 1 colum. longa, transversa, pl. 4 patal., crassis, brevibus, non parallelis (1. 4. parvis), tuberculo crasso in marg. exter., perist.

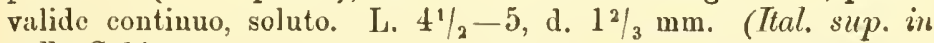
valle Sabia). Cat. moll. prov. Brescia 1851, 1852 c. f. Poll. l. c. 1887 c. f.

C. ligustica Poll. T. anguste umbilicata, cylindrica, lavis, apice subattenuato; anfr. 10, ult. circa umbil. obtuse carinatus, ap. subtriaugularis, infra subangulata, lam. 1 par. validiss., emersa, longiss., pl. 1 colum, valida, supera, transversa, pl. 3 (4) palat. brevibus, profundis, gracilibus, infra punctif., et dente conico in marg. exter. L. $41 / 2-5, \mathrm{~d} .11 / 2 \mathrm{~mm}$. (Itcl. ad Genua). L. c. 1887 c. f. (Poll.)

\section{(6.)}

C. biplicata Mich. T. cylindrica, fere lævigata, nitida, lutescens v. rufescens; anfr. 8-9, ult. basi subcompressus, non ascendens; ap. oblonga, supra latior, callo par. tenui, lam. 1 par. longa, emersa, extus interdum denticulo profundo, pl. 1 colum. longa, horizontali, emersa, supera, pl. 4 palat. (1. 4. minimis, profundiss., 2. valida, 3. breviore, extus productis). L. 5, d. $2 \mathrm{~mm}$. (Gallia merid.). Compl, 1831 c. f. Poll. l. c. 1887 c. f. - Forma 1) margidens W. (Binn. Conch. 1887), ap. margine exter. minus incrassato. sat dente conico prædito (It. ad Firenze).

Var. toscnuice (B.) Poll. l. c.: t. pallidior, ap. magis verticalis, callo par. obsoleto, denticulo supplementario in par. nullo, pl. palat. srepiss. 3. (It., Toscana).

Var. excessiva Gredl. (Tirols Conch. 1856): t. plerumque longior et gracilior, albido-hyalina, ap. lam. et plicis typicis, sed brevioribus, magis immersis, denticulo par. validiore nec non plica altera minuta ad basin col.; $1.41 / 2-5$, d. $1 \frac{1}{2} \mathrm{~mm}$. (Tirol, Carinth.) Poll. l. c. $1887 \mathrm{c.}$. 
Var. Iocurali (B.) Loc. (Conch. Fr. 1894): t. exacte eylindrica, apice rotundato, anfr. $7-8$, planulati, ap. infra paullo angustior, lam. 1 par., elongata, pl. 1 colum. magna submarginali, pl. 3 palat. (1. 3. rudimentalibus, media longa, emersa); 1. 4 $1 / 2$, d. $1^{3} / 4 \mathrm{~mm}$. (Gallia, Alp.-Narit.)

\section{Gen. P a g o d i i a Stab. (Moll. Piem. 1864).}

P. pagodula Desm. T. cylindrico-ovoidea, sat ventrosa, tenuis, corneo-fulvida, anfr. ult. fortius et distantius costata; ap. sinuoso-subquadrata, marg. exter. medio impresso, angulatim producto et intus incrassato. L. $31 / 2-4$, d. $2-21 / 4 \mathrm{~mm}$. (Alpes omnes, Morea, Transcaucas.). Bull. soc. Bord. 1830 c. f. le. f. 325 . - Forma 1) gracilis Btt., 1. $3^{1 / 3}-3^{2 / 3}$, d. $1^{1 / 2}-1^{3 / 4} \mathrm{~mm}$. (Dalm., Messina, Montenegro).

Var. subdola Gredl. (Tirols Conch. 1856): t. cylindrica, apice late rotundato, rufobrunnea, argenteo micans, densius costulata, axi stricto (non curvato), tuberculo margin. levi; 1. 4, d. vix $2 \mathrm{~mm}$. (Tirolia).

Var. obliqua W. (Binn. Conch. 1887): t. obesa, ovata, prrecipue anfr. ult. distantius costata, anfr. ult. fere ad medium anfr. penultimi ascendens, ap. dextrorsum sat excentrica, marg. exter. profunde impresso; 1. 3, d. $1 \mathrm{~mm}$. (Carinth. ad Malborgeth).

Var. adиmiі W. 1. c.: t. anfr. ult. densiss. verticaliter striatulo, cæteris oblique et sat distanter costulatis, ultimo in medio fossula instructo, antice et postice ieque alto, ap. triangulari, superne latiore. (Ital. ad Edolo).

Var. lederi Bttg. ap. Radde (Fanna et Flora 1886 c. f.): t. spira magis conico ovata, acutiore, dense acuteque costulatostriata, anfr. minus convexis, ult. vix impresso, altius ascendente, ap. marg. exter. minus sinuato; $1.31 / 2$, d. $2 \mathrm{~mm}$. (Lenkoran).

Var. mastenimaa Nev. (Proc. Lond. 1880 c. f.): t. ovoideo-conica, corneo-flavida, sat distanter capillaceo-costulata; anfr. 8, primi 4 regulares, convexiusculi, cæeteri convexi, ult. sicut belobatus, ascendens; ap. obliqua, subtriangularis, basi rotundata, dente in marg. exter.; 1. $3^{1 / 2}$, d. $2 \mathrm{~mm}$. (Gallia, Alp.-Marit.). Loc.

Var. perplicutn Sterki (Nachr. blatt 1894): t. superne costulis modice densis, in dimidia ultima anfr. ult. multo dentioribus; ap. plicis palat. multis profundiss. (tantum pelluciditate testre conspicuis) prædita: snb finem inter num $\mathrm{pl}$. typicæ, quam validam habet, sunt pl. 2. brevior sed sat fortis plice colum. forti opposita, et profundius super illam pl. 3.; pone sut. pl. 4. tenuis; profundiss. in pariete sunt 2 lamella, finibus suis in ap. subconspicure. (Gallia ad "Payence").

Var. Ionuranigunti Cout. (Bass, du Rhone 1881): "t. umbilicata, globoso-cylindrica, elegantiss. lamellicostata, obtusiss.; anfr. 5, valde convexi, sut. profunda, primi rapidi, duo ultimi subæquales, ult. leviter minor; ap. pl. palat. remo- 
tissima, pl. spiralis valiıla, translucens, ap. fere attingens, marg. valde approximatis. L. 1//10, d. ${ }^{95} / 100$ mm.": Cout. (Gallia in valle Rognac). Jueenis? I'. tschapecki Gredl. Nachr. bl. 1877) t. juvenis est!

\section{Gen. S a ll d a h I i a W. (Binn. Conch. 1887).}

S. cylindrica Mich. T. anfr. 11-12, ap. rotundatoovalis, lam. 2 par. (angul. longa, superne duplicata), pl. 2 colum. profundis, pl. 4 palat. (1. perbrevi, profundiss., exteris longis emersis) et $\mathrm{pl}$. basali immersa. L. $8, \mathrm{~d} .31 / 3 \mathrm{~mm}$. (Pyrenace). Bull. soc. Bord. 1829 c. f. Ic. f. 643. - Forma 1) cchtrodes W. (Binn. Conch. 1887, M. T. Ilist, t. 27 f. 24), perist. pliculis multis intermixtis; 2) longa M. T. (1. c. f. 25) t. elongata, anfr. 14, ap. lam. et plicis 8-9; 3) curtu M. T., brevis (1.5-6 mm.), anfr. 8-9, perist. dense plicatulo.

Var. cylindriformis (B.) Loc. (Conch. Fr. 1894): t. cylindrica, perlonga; anfr. 12-13; ap. ovali-oblonga, lam. 2 par., pl. 2 colum. (inferiore breviore et ninus immersa), pl. 4 palat. (1. rudimentali, 2. maxima, sola emersa, 3. 4. brevibus; $1.8 \frac{1}{2}-10$, d. $21 / 2-3 \mathrm{~mm}$. (Pyren. orient.)

Var. corrugata Loc. l. c.: t. cylindrico oblonga, sursum rapide attenuata; anfr. 11-12; ap. ovali-oblonga, lam. 2 par., pl. 2 colum. emersis cum pliculis $2-4$ in perist., pl. 4 palat., quarum 3 emersis, cum pliculis $3-4$ in perist. incrassato; 1. $7-8$, d. $2^{3 / 4}-3 \mathrm{~mm}$. (Gall., Villefranche).

Gen. P u p a Drap. (Hist. 1805).

(Torquilla Faure-Big. ap. Stud. 1820).

Conspectus.

$+T$. cinerea, fusco-nebulosa; ap). edentata.

†† 'T. cornea $v$. castanea, concolor.

1. T. cylindrica, conoideo-acuminata (l. 6-8, rariss. 10-13 nim.); ap. pl. typ. 2-2-5, colum. brevibus, subcqualibus, profundis, palat. (cxc. 1.) longis, emersis. - Eur. merid. (2.)

2. T. subturrita, longe attennata, lavigata (l. 10-18, raro 6-9 mm.); ap. pl. typ. 2-2-4, colum. dentif., supernis, immersi", perist. crusso. - Gall., Hisp., Helv. (3.)

3. T. gracilis, longe attenuata (l. 10-13 mm.); anfr. 10-15; ap. plicis 2-2-5, colum. vix conspicuis, palat. 3 inferis longiss, medio subabbreviatis. Pyrencea. (4.)

4. T. gracilis, cylindrico-fusif. (l. 8-9, raro 10-12 mm.); anfr. $9-14$; ap. pl. numero variis $(2-2-3$ ad 6-3-5), colum. 1. longiss., emersa, et sape pliculis minutis accessoribus, perist. subcontinuo. Pyren., Hisp. (5.) 
5. T. gracilis, subturrita (l. $6-8 \mathrm{~mm}$.), anfr. $S-10$, ap. pl. 2-2-3 (4), colum. 1. v. etiam 2. longis, palat. 2-3 inferis longis, emersis, medio abruptis, perist. callo valido continuo v. soluto. - Gall., Pyren. (6).

6. T. cylindracco-oblonga (l. 7-10 mm.); anfr. 9; up. pl. 3 (4)-2-4 (5), et perist. undique crebre pliculoso, colum. subcmersis, intus altis, extus tenuibus. Gall., Hisp., Aly. (7.)

\%. T. cylindraceo-oblonga (l. 6- $7 \mathrm{~mm}$.$) ; anfr. S-10,$ ult. basi rotundato; ap. pl. 2-1-4, angul. tubcrculo forti margini adharente, colum. breviss. $v$. inconspicuo, pl. palat. 1. immersa, longa, 2.3. longis, 4. breviss. - Gall, Hisp., Lusit., Alg. (8.)

8. T. cylindracea (l. 6-8 mm.); anfr. $9-10$; ap. $p l$. 2-2-4 et denticulo in utroque angulo, $\mathrm{pl}$. colum. 1. immersa, 2. longa, emersa, palat. 1. immcrsa, breviss., 2. 3. emersis, 4. breviore. - Pyrenaa. (9.)

9. T. ovoideo-oblonga (l. 5-9 mm.); anfr. 9-10; ap. pl. 2--2-4 (2-2-2 vel 4-3-4), angul. longa, sape extus duplicata, colum. intus fortibus, 1. cxtus producta, patal. 1. parva, immersa, exteris cmcrsis. Eur. merid. (10.)

10. T. ovoideo-conica ( $7.4-7 \mathrm{~mm}$.), ventrosa; anfr. $7-9$; ap. pl. 3-2-3 (4-2-4), omnibus v. plurimis longis, emersis (sepe undique pliculis accessoribus), perist. subcontinuo. - Pyreną. (11.)

11. T. fusif. (l. $13-14 \mathrm{~mm}$.); anfr. 12-13; ap. pl. $2-2-2$ (4), colum. parvis, profundis, palat. mediis emersis. - Dalm. (12.)

12. T. oblongo-conica (l. $5 \mathrm{~mm}$.); anfr. $8-9$; ap. $p l$. $2(1)-2-4$, colum. supernis, longis, palat. 2. 3. 4 . longis, callo par. tenui. - Dalm. (13.)

13. T. cylizulricu (l. $4 \mathrm{~mm}$.); anfr. 7; ap. $p l .1-2-4$, colum. breviss., inmersis, palat. 2. 3. elongatis. Gracia. (14.)

14. T. cylindriea (l. 5-7); anfr. 8-9; ap. pl. 2-3-4, omnibus (preter palat. 1.) elongatis, nullis emersis, palat. (exe. 1.) intus aquis, non medium anfr. attingentibus. - Gall. merid. (15.)

15. T. subturrita (l. $6-7 \mathrm{~mm}$.); anfr. 9-10; ap. $p l$. 2-2-4, colum. profundis, brevibus i2. paullo longiore), palat. 1. e nodis 2, extus et intus, suppleata, catcris intus ad medium anfr. produrctis et pliculis auctis, 2. 4. medio longe abruptis, 3. tenui, emersa. Gall. (16.)

16. T. cylindraceo-oblonga (l. $11 \mathrm{~mm}$.); anfr. 10; ap. proter dentem 1 in callo par. edentata. - Syria. (17.)

17. T. cylindraceo-fusif. (l. 11-12 mm.); anfr. 11; ap. pl. palat. 2-4, de catero edentatu. - Gallia. (18.)

18. T. obcso-oblonga, ventricosa (l. $6-7 \mathrm{~mm}$.); anfr. 8-9, ult. alte ascendens; ap. 2-2-3, fere omnibus longis, emersis, callo par. valido pliculis $7-S$ obsito. - Alger. (19.)

19. "T. sccali persimilis". - Afghanistan. 
P. similis Brug. T. cylindrico-fusif, sp. longe attenuata, apice pertenui, acuto, cinerea, fusco-nebulosa et strigata, dense striata; anfr. 8-10, sut. immersa; ap. lam. par. 2 (exteriore parva, subangul.), pl. colum. 2, superæ, transversæ (1. valida, 2. parva), pl. palat. 2, longæ, emersæ; perist. rectum, acutum. L. 12, d. $4 \mathrm{~mm}$. (Gallia merid., Ital., Sicil., Corsica.). Encycl. 1792. Ic. f. 336, 945. - Forma 1) major, 1. 16, d. 41/2 mm.; 2) minor, 1. 9-10, d. $3 \mathrm{~mm}$; 3) variegella Jan (Cat. 1832), t. major et fortius variegata; 4) pachygastra W., t. ventricosa; 5) lavigata W., t. irregul. striata v. lovigata.

Var. dissimilis W. (Binn. Conch. 1887): t. omnino cylindrica, tantum in apice breve conica, anfr. subæquales, lentiss. crescentes, ad sut. angulati, ap. dente angul. nullo, lam. par. valida, perobliqua; $1.12-14 \frac{1}{2}$, d. vix 3 , ap. 1. $21 / 4 \mathrm{~mm}$.

Var. porcellatn W. (Binn. Conch. Suppl. 1890): t. subfusif., medio sat ventricosa, superne forte attenuata, peracuta, regul. dense costulato-striata; 1. 11, d. medio $3 \frac{1}{2} \mathrm{~mm}$. (Ital. ad Spezzia).

Var. olivetormm Loc. (Conch. Fr. 1894): t. longe tenueque attenuata, anfr. 10-12, convexiusculi, ap. lam. 2 par. et pl. 2 palat. (col. tantum pliciformi); $1.15-16$, d. $3 \mathrm{~mm}$. (Gallia merid.

Var plagionixa Loc. 1. c.: t. fere regul. conica, anfr. 8-9, subplani, ult. ad basin sat carinatus, ap. subrectangularis, lan. et pl. typicis; 1. 9-10, d. 3//2 mm. (Gallia merid.)

P. pallida (Phil.) Rm. T. subfusif,, acuta, violascenticinerea, subunicolor, apice regul. attenuato, sublente tenue striatula; anfr. 8, sut. tenui; ap. edentata, perist. patulo, acuto, marg. æque curvatis, col. inferne recta, obliqua, ad parietim plus minusve, sæpe forte, callosa. L. 10, d. $3 \mathrm{~mm}$. (Italia media). Ic. f. 732 .

Var. nunicia Parr. ap. Pfr. (Mal. Blätt. 1854): t. major, medio sæpius cylindrata, lam. parvis 2 in par.; l. 10--13, d. 3//2-4 mm. (Gall. merid., Ital. super.) Ic. f. 944.

P. psacolena B. T. fusif-turrita, acuta, cinereo- et fusculo-strigata, subtiliss. striata; anfr. 6, convexi, ult. circa foramen infundibulif. compressiusculus; ap. ovalis, edentata, perist. recto, acuto, marg. subparallelis, superne conniventibus. L. $7^{1 / 2}$, d. $3^{1 / 2} \mathrm{~mm}$. (Gallia, Alp.-Marit.). Amén. mal. 1859. Ic. f. 929 .

P. fiumentum Drp. T. ovato-cylindrica, lutescens; anfr. $8-10$, ult. pone ap. albocallosus, basi subcompressus; ap. se- 
miovalis, pl. angulari extus crassa, pal. 1. remota, tenui, brevi, cæteris longe intrantibus, 4. maxima, inferis medio gibbosis, perist. albo, expanso v. reflexo. L. $7-8$, d. $23 /-3 \mathrm{~mm}$. (Grall., Hisp., Helv., Germ., Belgia, Ital., Austria, Serbia, Dalm.). Tabl. moll. 1801. Ic. f. 34, 310. - Forma 1) castanea Gredl. (Nachr. blatt 1879), t. intense rufobrunnea (Tirol.); 2) intrans n., ap. plicis palat. 2. 3. 4. intus usque ad marg. colum. porrectis (Lyon).

Var. elongata Rm. (Ic. f. 313): t. subcylindrica, elongata, acuminata, callo cervicis nullo; ap. semicircularis, lam. et plicis validis; 1.8, d. $3 \mathrm{~mm}$. (Triest, Carinthia \&c.).

Var. illyrica Rm. (Ic. f. 312): t. subcylindrica, sublævigata, sp. elongata; anfr. planiores, callo cervicis obsoleto v. nullo; ap. plicis tenuioribus, profundioribus; 1. 10-11, d. $4 \mathrm{~mm}$. (Illyria, Ital. super., Transsylv.) - Forma 1) minor Rm. (Ic. f. 311), t. gracilis, ap. plicis palat. imperfectis, sæpius tantum duabus mediis præsentibus, l. 7 , d. $2 \frac{1}{2}-2 \frac{3}{4} \mathrm{~mm}$.

Var. curta Kstr. (Mon. 1845 c. f.): t. parva, ventricosa, regul. striata; anfr. 8 , ult. callo præditus; ap. plicis validis; 1. $6-7$, d. $3 \mathrm{~mm}$.

Var. cylindracen Z. ap. Rm. (Ic. f.315): t. cylindrica, medio lateribus parallelis, apice acuminato conica, glabiuscula, corneo-lutescens; anfr. ad sut. subangulati; ap. semiovatorotundata, pl. 2-2-4, marg. exter. labiato, reflexo; l. 13, d. $3 \% / 4 \mathrm{~mm}$. (Dalm.)

Var. primula W. (Nachr. blatt 1892): t. elongata, omnino cylindrica, apice tantum breviter conico-attenuato, albida, dense regul. striatula; anfr. 11, superi lente, tres ante ult. aque lati ac alti, ult. parvus, latere exter. subtriangulari; ap. lam. ang. vix conspicua, par. profunda, tenui, elongata, pl. pal. 4 (1. profunda, tenui, 2. 4. tenuiss., vix conspicuis, 3. subemersa, longa); $1.9^{1} / 2$, d. $2 \mathrm{~mm}$. (Hungaria).

Var. pachygastra Z. ap. Rm. (Ic. f. 314): t. cylindricofusif., medio ventricosa, utrinque lente attenuata, costulatostriata; anfr. 11, ap. pl. angulari longa, supra elata, flexucosa, cum marg. conjuncta, palat. longis, 1. pone ad sut., 2. tenui, breviss., cæteris submarginalibus; $1.11-12$, d. $3 \frac{1}{2} \mathrm{~mm}$. (Dalm., Bosnia, Tirolia).

Var. Apennina Ch. ap. Kstr. (Mon. I845 c. f.): forma et sculptura præcedentis; anfr. 10; ap. pl. 3-2-5, validis, angul. longa, extus incrassata et conjuncta, intus plicula juxtaposita parva, palat. longis, præter 1 marginalibus; 1. 9-10, d. $3 \mathrm{~mm}$. (Ital. super.)

Var. hungarica Kim. (Verh. siebenb. Ver. 1890): t. fusif., ventricosa, apice conico, nitens, rufescens, tenuiss. et densiss. regul. striata, anfr. ult. non vel parum callosus, ap. pl. 3-3-5, angul. sæpe incrassata, par. media peralta et forte compressa, exteriore (sub angul.) parva, profundiss., colum. 1. minima, pal. 1. brevi immersa, cæteris submarginalibus, intus tantum ad medium anfr. productis (raro inferis tenuiss, ad marg.); 1. 5\% $1 / 5$, d. $22 / 3-3 \mathrm{~mm}$. (Carinth., Görz, Triest, Ital., Hung., Rumænia, Transsylv.). 
Var. bugeysinck Loc. (Variat. 1. 1881): t. mediocris, ventrosula, perist. sat forte et reflexum, pl. colum. et palat. profunde immersæ, tantum 3. palat. distincta et emersa (Gall.)

Var. rhodtanica Loc. (Conch. Fr. 1894): t. ovoidea, brevis et obesa, rapide sursum attenuata, tenue regul. striata, corneo-rufa; anfr. 8, convexi; ap. subrotunda, pl. 2-2-4, angul. longa, supra incrassata, pal, inferis 3 , emorsis, perist. reflexiusculo, crasso; 1. 6-7, d. $3 \mathrm{~mm}$. (Gallia).

Var. rustica (B.) Loc. 1. c.: t. cylindracea, sursum lente attenuata, tenuiss. striata, cornea; anfr. 7-8, convexi; ap. subrotunda, pl. 2-2-3: angul. recta, callosa, par. minore, immersa, obliqua, colum. profundis, parvis, palat. 1.2. parvis, immersis, 3. emersa; perist. expansum, reflexum, sat tenue; l. $7-8$, d. $21 / 2 \mathrm{~mm}$. (Gallia).

Vur. crianoda (B.) Loc. l. c.: t. breve cylindracea, lente attenuata, tenue striata; anfr. 9, convexi, superi angnsti, ult. magmus; ap. obliqua, semicircularis, pl. 2-2-3, par. perapproximatis, subequalibus, palat. 1. parva, remota, creteris emersis, perist. expanso, perreflexo et incrassato; $1.8 \frac{1}{2}$, d. $3 \mathrm{~mm}$. (Gallia).

Var. men (B.) Loc. l. c.: t. conoidea, ventricosa, brevis et obesa, cornea, vix striatula; anfr. 7, convexi; ap. basi rotundata, pl. 2-2-3, par. valde approximatis, rectis, subæqualibus, colum. sat fortibus, profundis, palat. 1. 2. minimis, 3. emersa, perist. sat expanso, reflexo, incrassato; 1.6, d. $23 / 4$ mm. (Gallia).

Var. nova (B.) Loc. l. c.: t. ovoidea, lente attenuata, corneo-rufa, vix striatula; anfr. 7 , sat convexi; ap. subovalis, pl. 2-2-3, angul. magna, emersa, colum. parvis, profundis, palat. 1. 2. valde immersis, parum conspicuis, 3. emersa, perist. sat tenui, parum expanso; l. 6, d. 2\% $\mathrm{mm}$. (Gallia).

\section{(3.)}

P. Variabilis Drap. T. corneo-rufa; anfr. 9-10, subplaniusculi, ult. antice albus; ap. semiovalis, pl. angul. valida, brevi, par. longa, immersa, palat. medio gibbosis, 1. 2. brevibus, immersis, 3. elongata, emersa, 4. fere punctif., perist. expanso, forte albolabiato, marg. distantibus. L. $10-12$, d. $3-31 / 2$ mm. (Gallia, Hisp., Helv.). Tabl. 1801. Ic. f. 721. - Forma 1) minor, 1. 6-8, d. $2^{1 / 2}-3 \mathrm{~mm}$.

Var. polita Risso (Eur. mer. 1826 c. f.): t. magna, subturrita, lente attenuata, rufa v. corneo-Iutescens, nitidiss.; anfr. 12-13, lentiss.; ap. infra paullo attenuata, pl. 2-2-4, angul. subrecta, par. immersa, arcuata, palat. 1. 5. rudimentalibus. 2. brevi, 3. emersa, perist. reflexo, albo, incrassato; l. $15-18$, d. $3^{3} / 4-4 \mathrm{~mm}$. (Gall. merid.)

Var. sabaudina B. (Mal. Aix-les Bains 1864 c. f.): t. fortius attenuata, rufa v. rufescens; anfr. 9; ap. subrotunda, pl. typicis, angul. longa, flexuosa, sat callosa, palat. 1. 2. 4. profundiss., rudimentalibus, 3 . emersa; 1. 7-9, d. $2^{3 / 4}-3 \mathrm{~mm}$. (Gallia, Sard.) 
Var. ovulint Loc. l. c.: t. ovoideo-ventricosa, utrinque attenuata, rufescens; anfr. 8 ; ap. rotundata, pl. $2-2-3$, angul. forte flexmosa, colum. sat fortibus, profundiss., palat. 1. 2. rudimentalibus et profunde immersis, 3. emersa, perist. sat tenui; l. $61 / 2-8$, d. $3 \mathrm{~mm}$. Gallia). P. multident. v. ovularis M. T.?

Var. isclureostoma (B.) Loc. l. c.: t. subconica, basi sat lata, sursum regul. attenuata, rufescens, tenue striata; anfr. 9, subrequales, fere plani; ap. semirotunda, pl. 2-2-3, angul. brevi, perobliqua, colum. minutis, valde insqualibus, palat. 1. 2. rudiment., 3 . parva at marginali, perist. sat tenui; 1. 9, d. $3 \mathrm{~mm}$. (Gallia).

Var. elsodunerisis B.) Loc. 1. c.: t. cylindrica, anguste elongata, ad summum rapide attenuata, sat forte striata; anfr. 10, sat angusti; ap. anguste ovalis, basi retusa, pl. 2-2-3, colum. sat fortibus, palat. 1. breviss., 2. rudiment., 3. emersa, perist. reflexo, sat crasso; 1. 11, d. $3 \mathrm{~mm}$. (Gall., Embrun).

Var. arctespira (B.) Loc. l. c.: t. ut præcedens, anfr. 10-11, lenti, superi convexi, inferi fere plani, ap. semilotundata, pl. numero typicis, angul. vix obliqua, par. immersa, forte arcuata, colum. fortibus, vix conspicuis, palat. 3. emersa, longa, tenui, ceteris rudimentalibus, perist. reflexo, valde crasso; 1. $10-11$, d. $2^{3 / 4} \mathrm{~mm}$. (Gallia merid. .

Var, angelsaxis IV. (Binn. Conch. 1887): t. cylindracea, tantum superne conica, sut. fusco-marginata; ap. infra producta, pl. typicis, angul. superne forte curvata, conjuncta, palat. 1. brevi, 4. punctif., 2. 3. emersis, 2. leviore, perist. reflexo, valde convexo-labiato; $1.9-11$, d. $2^{1 / 2}-3^{1 / 2} \mathrm{~mm}$. (Gallia, Poisat).

Var. delphinensis Loc. 1. c.: t. anguste cylindrica apice breviter angustato, pallide cornea, nitidiss.; anfr. 12-13, lentiss.; ap. parva, semirotundata, pl. typicis, angul. forte arcuata, par. immersa, forti, curvata, colum. parvis, palat. 1. 2. sat fortibus, immersis, 3. emersa, 4. ludiment., perist. expanso, reflexo, sat tenui; 1. $11-13$, d. $2^{3 / 4}-3 \mathrm{~mm}$. (Gallia).

Var. plagiostoma (B.) Loc. 1. c.: t. conica, ventricosa, lente attenuata, rufescenti-coruea; anfr. $7-8$; ap. late ovalis, obliqua, pl. 2-1-2, angul. parva, par. majore, arcuata, palat. subrequalibus, 2. submarginali, perist, expanso, crassiusculo; 1. 10, d. $3_{/ 2}^{1 / 2} \mathrm{~mm}$. Gallia).

P. obliqua Nev. T. anguste cylindrico-elongata, lente attenuata, cornea, nitida, lævis; anfr. 11, subæquales, fere plani, sut. marginata; ap. ab axi perobliqua, subquadrangulata, marg. obliquis, subparallelis, callo par. valido, pl. $2-2-3$, colum. parum immersis, palat. media sat longa, stricta, emersa, perist. crassato. L. $12-15$, d. $3-31 / 2 \mathrm{~mm}$. (Gallia merid.) Proc. Lond. 1880 c. f. Nev.

P. limonensis W. T. subcylindraceo-fusif., sublævis, nitida, cornea; anfr. 9, vix convexiusculi, ult. antice albidus, basi compressiusculus; ap. semielliptica, pl, $2-2-3$, angul. 
modica, colum. minimis, profunde immersis, vel inferiore extus producta, palat. omnibus longis, validis, emersis, intus longiss. elongatis. L. ad 8, d. $21 / 2 \mathrm{~mm}$. (Italia super. ad Timona). Binn. Conch. 1887.

Var. simoni Bttg. (Nachr. blatt 1884): t. minor. ap. magis subcircnlaris, minus valide labiata, lam. angul. humiliori, pl. palat. minus profunde intrantibus; $1.6-61 / 2$, d. $21 / 4$ $-2 \frac{1}{2}$ mm. (M. Cenis).

P. mortilleti Stab. T. ut præcedens; anfr. 8, convexiusculi, ult. basi rotuudatus; ap. semiovata, pl. $2-2-2$, angul. punctif. vel tenue breviterque producta, colum. dentif., inferiore fortiore, palat. 1. 3. punctif. ad finem interiorem pl. 2. immersis, 2. multo longiore, tamen non emersa, inarg. æque curvatis. L. 61/2, d. $\mathrm{I}^{1 / 2} \mathrm{~mm}$. (Ital. superior, Gallia ad Briançon). Moll. Piem. 1864.

P. affinis Rm. T. cylindrico-fusif., flavo-cornea; anfr. $10-12$, lenti, medii 4 ante ult. subrquales, sat convexi, ult. major, basi compresso-cristatus; ap. subovata, pl. angul. longiss., forti, supra bifida $\mathrm{v}$. duplicata, par. interna, profunda, pone ad et circa col. arcuata, palat. 1. 2. profunde immersis, 1. suturali tenui, 2. brevi crassa, ceteris longiss., extus et intus crassis, medio tenuibus $\nabla$. abbreviatis, 3. emersa, ınarg. disjunctis. L. $10-11$, d. $2-21 / 2 \mathrm{mn}$. (Pyrencece). Ic. f. 642 .

Var. saeva W. (Binn. Conch. 1887): t. brunnea, obsolete costulato-striata, ap. pirif.-trigona, infra acute angulata, pl. $2-2-3$, palat. 1. submarginali, marg. colum. superne late reflexo, exter. superne forte arcuato; 1. 13, d. $3 \mathrm{~mm}$. (Gallia ad Luchon).

Var. freseriana Bof. (Bull. Fr. 1890): t. obesior, anfr. primis convexis, ceteris planiusculis, ult. valde ascendente, ap. ab axi obliqua, angustior, fere elongata, pl. 2-2-4, palat. 1. immersa, 2. valida, emersa, 3. minus producta, 4. parum immersa, callo par. tenui; $1.9-10$, d. $2^{1} / 3-2{ }^{2} / 3 \mathrm{~mm}$. (Gallia).

Var. perlonga Bof. 1. c.: t. gracilis, cylindrica, utrinque attenuata, nitida, corneo-rufescens, irregul. capillaceostriata; anfr. 13-15, sat rapide crescentes, sut. profunda, ult. irregul, rugosus; ap. anguste subelliptica, typice plicata, marg. recto-parallelis, reflexis, callo par. tenui; l. 13, d. $3 \mathrm{~mm}$. (Pyrenææ).

Var. phuthisica Bof. 1. c.: t. subcylindracea, gracilis, superne attenuata, in anfr. penultimo coarctata, nitens, corneofulva, confertim striata; anfr. 11-12, lente regulares, ult. exsertus, valde ascendens; ap. subovato-rotundata, typice plicata, callo par. distincto; 1.10, d. $21 / 3-21 / 2 \mathrm{~mm}$. (Pyrenææ). 
P. petrophila Fag. T. subcylindracea, supra anfr. penult. lente attenuata, tenue striata, fusco-cornea; anfr. 9, ult. basi retusus; ap. sat anguste ovalis, pl. $2-2-3$, sat validis, angul. longa, sæpe bifida, par. immersa, colum. 1. fere ad perist. elongata, 2. breviore, palat. subæqualibus, emersis, pl. colum. et lam. par. exacte oppositis, 2. majore (nec non ad sut. tuberculis $1-2$ immersis), perist. non vel subcontinuo, patulo. L. 8-9, d. 21/4 mm. (Pyrencece). Cat. Esera 1888.

Var. bofilli Fag. (Ann. mal. 1884): t. dense eleganter striatula, nitida, corneo-luteola, ap. minore, plicis minus robustis, perist. acuto, reflexo, albo, callo par. nullo; 1.8, d. $2 \mathrm{~mm}$.

P. andorensis B. T. cylindrico-fusif., sp. attenuata, argute striata, rufescenti-cornea; anfr. 10, ult. basi compressocristatus; ap. rotundata, pl. $6-3-5$, angul. 2 (1. parvula), par. 2 anticis fortibus et 2 internis, colum. 3. minima, palat. 1. 5. parvis, 2. 3. 4. fortibus, emersis, perist. albo, incrassato, expanso, marg. fere conjunctis. L. 10, d. $3 \mathrm{~mm}$. (Hispania). Rev. 1863 c. f.

P. lilietensis Bof. T. ut præcedens, sed minor, corneofulvescens, crista basali valida, acuta, alba; ap. truncato-ovata, pl. 5-3-5, angul. 2 validis, confertis (2. validiore), par. 2 externis prope lam. angul., 1 interna medio, palat. 1. parva, immersa, 3 medianis validis, emersis, 5. minutiss., marğ. exter. superne valde incurvato. L. $8-9$, d. $2 \mathrm{~mm}$. (Hispania). Bull. soc. Fr. 1886. (Bof.)

P. catalonica Bof. T. ut præcedens, sed ap. sæpe subtriangularis, basi angulata, ad dextram coarctata, pl. 9-11 (3 v. 5-2-4), angul. 2 validis, lamellif., sat approximatis (2. multo longiore), par. 3 (2 externis vix conspicuis, sæpe deficientibus), medio l immersa, colum. subhorizontalibus (1. emersa), palat. 1. valde immersa, reliquis validis, subemersis et intus valde productis. L. 11, d. $2^{1 / 2} \mathrm{~mm}$. (Hisp.) L.c. 1886.

P. hetara W. T. cylindracea, apice conico, dense regul. tenue at acute striata, distantius et validius in anfr. ult., fulvo-cornea; anfr. 10, regulares; ap. ovalis, pl. 2-2-4, angul. duplicata, colum. 1. longiss., emersa, palat. 1. profundiss., 2. 3. subemersis, introrsum ad marg. colum. prolongatis, callo par. nullo v. tenuiss. L. 8, d. 21/2 mm. (Pyrencece). Nachr. blatt. 1892.

P. montsicciana Bof, T. cylindr.-fusif, gracilis, rugulosa, corneo-fulvescens; anfr. 13-14, lente regulares, ult. valde rugosus, crista basali valida; ap. ovato-rotundata, pl. $3-2-3$, 
angul. duplicata, par. mediana in initio valida, deinde tenui v. interrupta, denique denuo valida, 3. dentif. sæpe duplicata juxta col., colum. l. magna, longa, externa, intus validiss., pal. 1. externa, 2. vix immersa, 3. basali, minore, lam. par. et p!. colum. intus valde approximatis, de cetero plicis multis accessoribus, callo par. sat valido. L. $9-121 /_{2}$, d. $3 \mathrm{~mm}$. (Hispania). L. c. 1890. (Bof.)

P. leptospira W. T. gracilis, cylindraceo-turrita, densiss. capillaceo-striata, fuscocornea, ad ap. incrassata, alba; anfr. 91: 11 , convexi, lenti, ult. ascendens, ad ap. contractus; ap. elliptico-rotundata, pl. $2-2-4$, angul, superne tenui du. plicata, colum. l. longiss., marginali, 2. breviore, palat. 1. breviss., immersa, 2. 3. emersis, perist. undique labiato, callo par. valido, albo. L. $7^{1} / 2-8$, d. $2 \mathrm{~mm}$. (Gallia). Binn. Conch. 1887.

P. migma W. Differt a præcedente t. infra medium cylindrica, sursum lente conico-angustata, lateribus convexiusculis, perforatione aperta, rotunda, anfr. inferis convexiusculis, ap. lam. angul. superne tuberculo munita, lam. par. immersa, pl. palat. 3, longis, emersis (1. 2. introrsum usque ad perforationem productis, 3. basali), callo par. acuto elato. I. 8, d. $2 \mathrm{~mm}$. (Pyrencece). Verh. Ges. Wien 1892.

P. attenuata Fag. T. præcedenti similis, sed conicoturrita, ab ap. usque lente et regul. attenuata, corneo-brunnea; anfr. 9, superi 6 lentiss. crescentes, inferi 3 multo altiores et æquales, superi 3 cylindracei, ceteri convexi, ult. postice celeriter attenuatus; ap. lam. angul. perlonga, pl. colum. fortibus, subæqualiter elongatis, longe tamen non emersis, perist. tenui. L. 7, d. $2 \mathrm{~mm}$. (Gallia). Bull. soc. Fr. 1886, W. in Binn. Conch. 1887.

P. vergnesiana (Ch.) Kstr. T. gracilis, cylindrica, tenue denseque costulato-striata, fuscocornea; anfr. 10-11, ult. basi cristatus, antice valde solutus; ap. obliqua, ovalis, soluta, pl. 2-2-3, angul. longa, par. brevi, obliqua, alta, colum. fortibus, brevibus, profundis, palat. 1.-2. interruptis, 3. medio impressa, 2. intus denuo valida et cum 3. usque ad marg. colum. productis, perist. albolabiato. L. $71 / 2-8$, d. $2 \mathrm{~mm}$. (Pyrencece). Hon. 1845 c. f.

P. pyrenaaria (Boub.) Mich. T. fere cylindrico-fusif,, rufescenti-cornea, dense regul. costulato-striata, ad ap. costulata; anfr. 9, ult. basi leviter compressus; ap. semiovalis, pl. 2-2-3, ut vergnes., sed palat. longis, marginalibus, perist. albo- 
incrassato, leviter expanso, marg. callo contiguis. L. $6-8$, d. $2-2 \frac{2}{3} \mathrm{~mm}$. (Pyrencece). Compl. 1831 c. f. - Forma 1) curtu M. T., minor, typice plicata, at plicæ minus distinctæ; 2) novemplicata M. T., ap. pl. palat. 5 (pl. 2 immersis).

P. clausilioides Boub. T. perf., fusif.-cylindrica, apice perobtuso, corneo-rufescens, leviter densiss. striata; anfr. 10, ult. ascendens, compressus, basi subcristatus; ap. subsoluta, pl. $2-2-2$ v. 3, angul, valida, bifurcata, par. parva, immersa, colum. compressis, immersis, palat. tenuibus, in medio interruptis (1. longa, subemersa, 2. remota, 3. brevi, immersa, sæpe deficiente, perist. continuo, soluto, albo, undique reflexo. L. 8, d. $3 \mathrm{~mm}$. (Pyrencece). Bull. hist. nat. 1835.

P. nansoutyi Fag. T. perf., subcylindrica, apice obtuso, conico, tenuis, translucens, corneo-rufa, regul. striata; anfr. 9, regulares, convexi, ult. basi subcompressus; ap. oblongo-rotuudata, pl. 2-2-2 (3), angul. brevi, par. immersa, colum. 1. inmersa, 2. vix conspicua, palat. 1. subemersa, 2. 3. immersis, perist. acuto, reflexo, marg. callo tenui junctis, exter. expanso. L. 7, d. 2 mm. (Pyrencex). Bull. Toul. 1880.

P. retracta $W$. T. perf., cylindrico-conica, densiss. striatula, pallide cornea; anfr. 8, convexi, lente regulares, ult. lente ascendens, basi rotundatus; ap. ovato-elliptica, pl. $1-2-4$ et tuberculo angulari, par. immersa, curvata, colum. breviss., profunde immersis, palat. immersis, parallelis, antice subæqualibus, 1. 4. breviss., 3. sat longa, perist. marg. disjunctis v. callo par. tenuiss. I. 8, d. 2 mm. (Hisp. ad Sevilla). Nachr. blatt 1892.

Var. semidens W. (Nachr. blatt 1893): t. fortius regul. striata, anfr. 7, ap. pl. palat. 4, longe et aque a margine remotis (1. 2. 4. equalibus, breviss., crassis, 3. paullo longiore), omnibus in plicas breves attenuatis. (Hisp. ad Albarraciu).

P. aulusensis Fag. T. cylindracea, vix acuminata $v$. conica, corneo-rufa, regul. et (præter in anfr. ultimis lævigatis) dense striata, sp. elongata; anfr. $91 / 2-10$, fere planulati, lente regulares, ult. major, ascendens, basi compressus; ap. oblongorotundata, pl. $2-2-3$, par. profunda, valida, colum. validis, remotiss., palat. 1. marginali, medio interrupta, 2. valida, paullo remota, 3. parva immersa, perist. coutinuo, crasso, ubique reflexo, leviter adnexo. L. 9 , d. $2 \frac{1}{2}-2^{3 / 4} \mathrm{~mm}$. (Gallia, Ariège). Moll. d'Aulus 1880 c. f.

P. occidentalis (Fag.) T. cylindracea, sursum lentiss. attenuata, obtusa, corneo-brunnea, nitens, ubique dense regul. eapillaceo-striata; anfr. $9-9^{2} / 2$, convexi, lente regulares, ult. 
infra suico longitudinali, ad basin obtuse cristatus; ap. quadrangulari-rotundata, pl. $2-2-4$, par. subæqualibus, colum. transversis, immersis, 1. sat forti, 2. vix conspicua, palat. 1. punctif,, immersa, 2. 3. emersis, 4. brevi, basali, antice pliculis alternantibus, perist, contimuo, soluto, albo, vix patulo. L. $6^{1 / 2}$ -7 , d. $1^{1 / 2}-2^{1 /} \mathrm{mm}$. (Pyrencece). Cat. Esera 1888, W. in Verh. Ges. Wien 1892.

P. polyodon Drap. T. ovato-oblonga, apice brevi conif., subtiliss. costulato-striata, pallide cornea; anfr. 9, ult. paullo attenuatus; ap. oblongo-subrotundata, pl. 3-2-4, angul. 2, infera longa, palat. 1. breviss., immersa, 2. 3. 4. longis, emersis, marg. disjunctis. L. $81_{2}^{1}-10$, d. $3-3 / 2$ min. (Gallia meril., Hisp., Algeria). Tabl. 1801. Ic. f. 326. - Forma 1) attrita M. T., ap. pliculis in par. obsoletis v. nullis; 2) rentricosa L., t. minor, ventricosa.

Var. ameliae (B.) Loc. (Conch. Fr. 1894): t. ovoidea, obesa, ventrosa, sursum rapide attenuata, corneo-rufescens; anfr. $7-8$, ap. pliculis $7-8$ in peristomate; $1.61 / 2-7$, d. 21/2 mm. (Gallia).

Var. ringicald (Mich.) Kstr. (Mon. 1845 c. f.): ap. basi angulata, pl, validis, par. 4 una in utroque angulo et 2 in medio), colum. longiss., emersis, palat. 3 , emersis, perist. undique vel tantum ad sinistrum pliculato; $1.71 / 2-8$, d. $23 / 4$ $-3 \mathrm{~mm}$. (Gall. merid., Alg.). Ic. f. 727.

P. montserratica Fag. T. cylindracea, sat fusif., gracilis, nitida, argute striatula, rufocornea, sp. elongata, lente acuminata; anfr. $91 / 2$, ult. ascendens, basi subcompressus; ap. oblongo-truucata, plicis ut polyodon, at tenuioribus, marg. exter. arcuato, colum. fere recto, superne expanso, callo par. tenuiss. L. 9, d. $21 / 4$ mm. (Hispania). Ann. mal. 1854.

P. brauni $\mathrm{Rm}$. T. ovato-oblonga, lutescens, minutiss. striatula; anfr. 8 , convexi, ult. basi rotundatus; ap. basi rotundata, pl. 2-1(2)-4, angul. longa, supra incrassata, margini adhærente, colum. profunde immersa, dentif., interdum subtus denticulo minuto, palat. 1. 4. immersis, (1. longe intrante, 4. fere punctif.), 2. 3. emersis, longe intrantibus, perist. patulo, crasso, albolabiato. L. $6-7$, d. $2 \frac{1}{2} \mathrm{~mm}$. (Gall. merid., Hisp., Lusitaria, Algeria). Ic. f. 726. - Forma 1 major, 1. 8, d. 3 mm.; 2) crassilabrum B., perist. late et valde incrassato.

Var. conispira W. (Nachr. blatt 1893): t. a basi lata et subtransversali sensim elongato-conica, acuta, virescenti-alba, 
dense regul. costulata, anfr. $81 / 2$, lentissimi, ap. omnino sub dimidiam externam basis posita, lam. angul. minima, par. longa, pl. colum. breviss., valida; l. 6 , d. ad basin $3 \mathrm{~mm}$. (Hisp., Albarrasin).

P. cristella W. T. oblongo-cylindracea, lutescens, eleganter costulato-striata; anfr. 9-10, lenti, superne convexi, ult. lente ascendens, medio sulculo longo et infra altero latiore longitudinalibus, basi in cristam fortem curvatam compressus; ap. semiovalis, infra angustior, at rotundata, pl. $2-2-4$ et denticulis $1-2$ in utroque angulo parietali, colum. 1. immersa, brevi, alta, 2. longa, horizont., emersa, curvata, sæpe extus plicula adjacenti, palat. 1. profunda, breviore, ceteris longis, 2. antice tumida, perist. late expanso. L. 6, d. $2 \mathrm{~mm}$. (Pyrenеєе, St. Saveur). Mal. Blätt. 1894 c. f.

P. partioti (Moq. Tand.) St. Sim. T. subcylindricoconica, elongata, acuminata, fuscocornea, regul costulata; anfr. 10, ult. prorsus alte ascendens, antice callosus, pallidus, basi forte cristatus; ap. semiovalis, pl. $2(3)-2-4$, angul. dextra crassa, extus bifida, sinistra tenui, louga, sæpe obsoleta, colum. horizont., 1. tenui, breviore, 2. fortiore, longa, emersa, palat. 1. profunda, breviss., ceteris longis, 2. marginali, 4 . basali, breviore, perist. reflexo, albo, incrassato. L. $8, \mathrm{~d} .2 \frac{1}{3} \mathrm{~mm}$. (Pyrenceœ). Misc. mal. 1848.

P. secale Drap. T. ovoideo-oblonga, conico-attenuata, rufo-cornea, tenue striata; anfr. 9, planulati; ap. semiovata, pl. 2-2-4, angul. longa, superne incrassata, conjuncta, colum. intus sat fortibus, 1. plus minusve producta, palat. 1. brevi, interna, ceteris subæqualibus, emersis, intus æqualibus, longe non medium anfr. attingentibus, perist. breviter expanso, albolabiato. L. $7-8$, d. $2-2^{1} / 2 \mathrm{~mm}$. (Gall., Hisp., Lusit., Ital. sup., Austria, Germ., Brit.). Tabl. 1801. Ic. f. 35, 317. - Forma 1) elongata Saulcy, t. longa ap. pl. 7 (pl. palat. 1. deficienti), 1. 9, d. $21 / 2-3 \mathrm{~mm}$; 2) minor, teniusenla, levigata, $1.61 / 2$, d. $1^{3} / \mathrm{mm}$.; 3) scligo Roth, ap. pl. palat. accessoribus prope col. (Bavar., Würtemb., Dalm.); 4) gracilior W., t. anfr. $91 / 2-10$, pl. palat. 1 . obsoleta; 5) cylindrica Loc. (Var. 1881), elongata, anfr. convexiusculi, pl. 9, l. 8-9 mm. (Gall.); 6) novemplicate B., pl. 3-2-4 (Gall., Tirol.); 7) decemplicata B , pl. 4-2-4 (Gall.); 8) oyonna:xia Loc., pl. 3-3-4 (Gall.); 9) duodecimcostata Loc., pl. 4-2-6, colum. 1. forti, palat. 1. immersa, 2. 3. validis, 4. 5. 6. pnnctif., remotis (Britan.)

Var. bourgeticn B. (Mal. Aix-les-Bains 1864 c. f.): ap. pl. 4-3--4, colum. 1. 2. fortibus, subæqualibus, subemersis, 3. parva, immersa; 1. 8, d. $2 \frac{1}{4} \mathrm{~mm}$. (Gallia) - Forma 1) sublavigati $B$. 
Var. phyma ta W. (Binn. Conch, 1887): t. eleganter capillaceo-striata, in anfr. ult. fortius et distantius, rufofulva, ad ap. albida; anfr. ult. tuberculo albo ad basin, perist. undique incrassatum, album, anguste reflexum, pl. 2 (et denticulo iin utroque angulo) - 2 (horizont., elongatis v. 1. emersa, 2. brevi) - 4 (mediis longis, emersis, intus elatis, truncatis, deinde tenue breviterque attenuatis); l. 7, d.2 mm. (Pyrenææ).

Var. gourdoninna Fag. (Moll. Pic du Gar 1882): t. ovoideo-oblonga, sp. regul. elongato-conica, corneo-rufa, dense regul. striata; anfr. 9, subconvexi, sat rapide regulares; ap. obliqua, oblonga, pl. 2-2-4, angul. marginali, extus duplicata, par. profunda, valida, colum. validis, subremotis, palat. 1. profunda, tuberculif., 2. emersa, 3. breviore, 4. remota, perist., crasso, reflexo; l. 8, d. 2-2/2 mm. (Pyrenææ).

P. boileausiana (Ch.) Kstr. T. subcylindracea, gracilis, sp. turrita rapide attenuata, tenuiss. capillaceo-striata, fuscocornea; aufr. 9-10; ap. obovalis, pI. 3(4)-2-4, angul. 2 a margine separatis, teuuibus, inter. longa, exter. breviss., sæpius plicula ad col., pl. colum. 1. longa, emersa, intus alta, palat. 1. brevi, interna, perist. marg. subæqualiter curvatis. L. $5-7$ (9), d. 2-21/4 $\left(2^{3 / 4}\right) \mathrm{mm}$. (Pyrencece, Ital. super., Brit.). Mon. 1845 c. f.

Var. fugrorum (Fag.) Loc. (Conch. Fr. 1894): t. fortius et paullo distantins striata, ap. basi magis retusa, lam. angul. ad col. distinctiore et semper præsente, lam. par. magis immersa; 1. 6-7, d. 2 mm. (Pyrenææ).

P. abrupta W. T. cylindraceo-fusif, tenuiss, regul. striata, pallide fulvida, sp. lente attenuata; anfr. 8-9, ult. basi convexus; ap. infra angustata, sed rotundata, pl. 2-2-4, angul. brevi, tenui, simplici, colum. 1. intus forti, extus producta, palat. 1. interna, parva, 2. emersa, medio abrupta, 3. 4. medio immersis, perist. tenui, simplici. L. $6 \frac{1}{2}-7$, d. $2 \mathrm{~mm}$. (Grallia ad Aulus). Binn. Conch. 1887.

P. piniana Fag. T. regul. conica, corneorufa v. violacea, dense striatula, sp. elongata, paullo acuminata; anfr. 8, vix convexi, regulares, ult. basi compressiusculus; ap. obliqua, oblonga, pl. $2-2-4$, ut secale, sed tenuioribus, minus approximatis, magis oppositis, angul. superne non incrassata, perist. acuto, tenui, reflexo. L. 7, d. $2 \frac{1}{4} \mathrm{~mm}$. (Gallia, Aulus). Moll. Aulus 1880 c. f.

P. oryzana Loc. T. breviter ovoidea, sat gracilis, lente attenuata, tenue regul. striata, sp. elongata, paullo acuminata; anfr. 8, vix convexi; ap. subrotundata, pl. 3-3-3, angul. 2, colum. 1. superna, interdum obsoleta, 2. forti, subemersa, 3. breviore, palat. subemersis, 2. paullo fortiore, perist. expanso, crasso. L. 6, d. $13 / 4 \mathrm{~mm}$. (Grallia). Conch. Fr. 1894. (Loc.) 
P. costata (Fag.) Loc. T. subconoidea, elongata, basin versus lente attenuata, rufo-violacea, nitens, sat distanter costata; anfr. 8, sat convexi, sut. profunda; ap. subovalis, basi retusa, pl. 2-2-2, angul. majori, tenui, arcuatula, colum. profundis, 1. majori, palat. subæqualibus, emersis, perist. vix expanso, incrassatulo. L. $6-7$, d. $2 \%$, mm. (Pyrencece). L. c. 1894. (Loc.)

P. Lasallei (B.) Loc. T. ovoidea, breviuscula et obesa, leviter attenuata, densiss. tenue striata, rufa, nitida; anfr. 8-9, convexi; ap. subovalis, pl. 4-3-4, angul. longa, extus ad marg. plicula et altera ad col. subrudimentalibus, colum. 1. 3. immersis, 2. emersa, palat. 1. 4. minimis, immersis, 2. 3. emersis, perist. reflexo, incrassatulo. L. $6-7, d^{2}, 2^{3 / 4}-3 \mathrm{~mm}$. (Gallia merid.) L. c. 1894. (Loc.

P. kraliki Let. T, cylindrica, elongata, cornea, nitida, dense eleganter striata, sp. brevi; anf 1.9 , ult. basi compressocristatus; ap. semioblonga, pl. $3-3-4$, angul. longiss. et plicula in utroque angulo, par. valida, immersa, colum. 1. forti, emersa, 2. 3. parvis, immersis, palat. 1. immersa, ceteris validis, approximatis, emersis, oppositis, perist. patulo, crasso, marg. conniventibus, tenue junctis. L. $61 / 2$, d. $21 / 2 \mathrm{~mm}$. (Gallia ad Douze). Mull. Lamalou 1877. (Let.:

P. ringens (Caill.) Mich. T. rotundato-perf, ovoideoventricosa, superne breve conica, rufescens, argute costulata; anfr. 8-9, lenti, ult. prorsus ascendens, ad sut. tumidulus, postice depressus, medio planatus, basi compressus; ap. ovalirotundata, pl. $4-2-4$, acutis, angul. perbrevi, par. emersis, longis, media extus subinterrupta, colum. longis, parallelis, emersis, palat. 1. punctif., immersa, ceteris lougis, interdum undique pliculis accessoribus, perist. tenui, subrecto, continuo. L. 5-6, d. $2 \frac{1}{2}-3 \mathrm{~mm}$. (Pyrencece). Compl. 1831 c. f. Forma 1) elongata H. T., gracile attenuata; 2) milchella M. T., t. parva, gracilis; 3) disjuncta M. T., t. marg. callo non conjunctis.

Var. fagotiana Loc. (Conch. Fr. 1884): t. vix ventrosula, anfr. ult. basi rotundata, ap. lam. angul. sinistra deficiente, marg. disjunctis, perist. crassusculo, expanso, reflexo: l. $6_{2}^{\prime \prime}$, d. $2 \frac{1}{3} \mathrm{~mm}$.

Var. subriugens Fag. (Moll. Pys. 1892): t. minute perf., elongata, gracilior, argute striatula, anfr. convexioribus, ult. basi magis compresso, ap. basi angustata, lam. par. 3, media immersa, extus sæpe plicula supplementaria; 1. 7, d. $21 / 2 \mathrm{~mm}$.

P. baillensi Dup. T. ovato-conica, subveutrosa, leviter lateribus convexis attenuata, tenue regul. striata, lutescens; 
anfr. 7, ap. semiovata, subquadrangularis, basi late rotundata, pl. 3-2-3, par. media profuuda, angul. longis, colum. 1 . horizont. longa, 2. arcuata, breviore, palat. 1. 2. emersis, 3. brevi, immersa, perist. vix patulo, incrassatulo, callo non $\mathrm{v}$. vix juncto. L. $4-5$, d. $2-2 \%$ mm. (Gallic merid.). Rev. Gers 1873 c. f.

Var. sarmunica Fag. (Bull. Toul. 1877): t. major, elongatior, minus acuminata, ap. pl. fortioribus, pariete magis plicato, pl. palat. superiore magis elongata et prominente; 1. $6 \frac{1}{2}$, d. $2^{3} / \mathrm{s} \mathrm{mm}$. (Montpezat).

P. fusiformis Kstr. T. elongata, fusif., subtiliss. striata, nitida, flavocornea; anfr. 13, angusti, ult. basi compressus, sut. albida; ap. semiovalis, alba, pl. $2-2-2$, perist. albo, labiato, reflexo. L. 13, d. $3^{2} / 3 \mathrm{~mm}$. (Dalm. ad Budua). Mon. 1845 c. f. Variat plica palat. 3. punctif. (Kstr.)

P. eximia W. T. subfusif,, a medio lente attenuata, leviss. striatula, infra lævigata, rufo-cornea, sut. filari; anf $11-12$, ultimi 4 subplani, ult. basi compressus; ap. pl. 2-2-4, angul. valida, par. tenui, immersa, col. superne et profundi æqualiter bidenticulata, palat. 1. brevi, 2. 3. subemersis, 4. basali rudimentali, perist. expansiusculo, crasse albo- v. carneo-labiato. L. 14, d. $4 \frac{1}{2}$ mm. (Dalmatia?). Mal. Blätt. 1875 ex descr, optima (P. fusiformis var.) Pfeifferi in Mon. IV p. 313 adnot.

P. appeliusi W. T. oblongo-conica, tenuiss. striata, corneo-brunnea, sp. elongata; anfr. $81 / 2-9$, superi angusti, iuferi lati, subæquales, sat convexi; ap. ovalis, pl. 2-2-4, angul. longa, extus duplicata, palat. 1. profundiss., ceteris longis, emersis, perist. albo, incrassato. L. 5 , d. $2 \mathrm{~mm}$. (Dalmatia). Verh. Ges. Wien 1892.

P. krüperi W. T. ápice conico, dense striatula, corneofulvida; anfr. $71 / 2$, convexi, sut. forte marginata; ap. ovalis, pl. $1-2-4$, par. mediana, sat magna, obliqua (pro angulari macula parva albida), colum. 1. parva, 2. minima, palat. a margine remotis, 1. 4. puuctif., profundis, 2. 3. mediocriter elongatis, perist. incrassatulo, marg. remotis, L. 4, d. $1 \mathrm{~mm}$. (Greccia, Kolokytha). Nachr. blatt 1893.

P. micheli Dup. T. cylindrica, superne lente attenuata, obtusa, rufocornea, superne tenue striatula, infra lævigata; anfr. 
8-9, angusti, subplani, ult. prorsus breve ascendens, basi leviter compressus; ap. ovalis, pl. angul. longa, antice libera et sæpe duplicata, colum. intus altis, 1. tenue producta, palat. 1. brevi, immersa, ceteris subemersis, crassis, perist. tenui, vix reflexiusculo, marg. approximatis, disjunctis. L. $5-6$, d. $1 \frac{3}{4}$ mm. (Gallia merid.). Hist. moll. I850 c. f.

Var. colanumella Loc. (Conch. Fr. 1894): t. anguste cylindrica, vix attenuata, rufa, sat regul., forte et paullo distanter striata; anfr. 8-9, sat convexi; ap. ovalis, pl. 1-2 -4 , par. media, immersa, forti, colum. breviss., profunde immersis, tuberculif., palat. immersis, 1.2. tuberculif., 3. paullo majore, 4. obsoleto; $1.6 \frac{1}{2}$, d. $1^{3} / 4 \mathrm{~mm}$. (Gallia merid.). Loc.

Var. oparea (B.) Loc. 1. c.: t. anguste clausilixe,, usque a basi lente attenuata, tenuiss. striata, rufobrunnea; anfr. 9-10, sat convexi, sat alti; ap. subtriangularis, basi angulata, pl. 2-2-3, angul. et par. tenuibus, colum. immersis, 1. majore, palat. 1. sola emersa, perist. subcontinuo, tenui; l. 10 , d. $2 \frac{1}{2} \mathrm{~mm}$. (Gallia merid.). Loc.

Var. olearum (B.) Loc. l. c.: t. cylindrico-oblonga, ventrosula, sat celeriter attenuata, tenuiss. striata, rufescens; anfr. $9-10$, ult. basi rotundatus; ap. ovalis, pl. $2-2-2$, angul. fortiore, colum. subæqualibus, immersis, palat. subæquis, 1. emersa, perist. subcontinuo, crassiusculo; l. 8, d. $2 \mathrm{~mm}$. (Gallia merid.). Loc.

Var. magdalenae (B.) Loc. l. c.: t. conoidea, lente a basi attenuata, nitida, sublævigata, corneo-fulvida; anfr. 8-9; ap. rotundata, pl. 2-2-3, par. et angul. subrqualibus, colum. 1. minima et profundiss., 2. forti et paullo elongata, palat. 1. minima, 2. emersa, 3. remota, perist. subcontinuo, paullo incrassato et reflexo; 1. 61/2-7, d. $2 \mathrm{~mm}$. (Gallia merid.). Loc.

Var. rusticula (B.) Loc. l. c.: t. conoideo-ventrosa, sat celeriter attenuata, corneo-rufa, nitida, lævigata; anfr. 8-9, sat convexi: ap. obovalis, basi magis retusa, pl.2-2-3, angul. duplicata, colum. fortibus, subæqualibus, immersis, palat. 1. emersa, 3. minima, perist. ut precedens; $1.6-7$, d. $2 \frac{1}{2} \mathrm{~mm}$. (Gallia). Loc.

Var. valconrtiana (B.) Loc. l. c.: t. cylindrica, brevis, celeriter attenuata, corneo-rufa, lævigata; anfr. 7-8; ap. obovatorotundata, pl. 2-2-3, angul. tenui, col rm. immersis, 1. majore, palat. 1. 2. emersis, 3. parva, perist. subcontinuo, patulo, vix incrassatulo; l. 6, d. $2 \mathrm{~mm}$. (Gallia). Loc.

P. anceyi Fag. T. "subfusif.-elongata" v. ab anfr. ult. gracile turrita, acuminata, corneo-rufa, obsolete capillaceo-striata; anfr. $9-10$, sat convexi, lente regulares, ult. ut micheli, sut. immersa; ap. oblongo-rotundata, pl. angul. nunc tenui, nunc tuberculif., perist. vix expansiusculo, sat tenui ("incrassato, undique expanso": Fag.). L. 6-7, d. 11/2-13/4 mm. (G'all., Marseille). Bull. soc. zool. 1881. 
P. libanotica Tristr. T. cornea v. albida, sub lente irregul. striata, apice perobtuso; anfr. 10, 1. -5. celeriter crescentes, 6. 7. subrequales, ultimi 2 valde angustati, ult. ad ap. contractus, basi cristatus; perist. reflexum, albidum. L. 11, d. $4 \frac{1}{2}$ mm. (Libanon ad Ainat.). Proc. Lond. 1865. (Tr.)

\section{(18.)}

P. bipalatalis W. T. cylindraceo-fusif,, dense striata, brunnea; anfr. 11, ult. basi compressus, sut. impressa; ap. angusta, oblonga, tantum pl. pal. 2, brevibus, remotis, perist. tenui, acuto, marg. remotis, exter. superne leviter arcuato, colum. oblique verticali, late reflexo. L. $11 \frac{1}{2}$, d. $22 / 3 \mathrm{~mm}$. (Gallia, Luchon). Nachr. blatt 1873.

P. eudolicha B. T. lanceolato-cylindracea, longe attenuata, rufescenti-cornea, argute striata; anfr. 12, fere plani, ult. major, basi cristatus; ap. pirif., paullo angulata, infra subcanaliculata, lam. angul. parva, de cetero superne intuenti edentata, sed profundissime existant et extus supra ap. plicæ 4 longæ albæ translucent, col. superne obliqua, marg. callo juncti. L. 13, d. $3 \mathrm{~mm}$. (Pyrencece). Moll. nouv. 1863 c. f.

P. letourneuxi B. T. medio sat ventrosa, argute forteque costulata, sp. lateribus forte convexis et apice brevi, obtuso; anfr. 8, lenti, ult. ad basin sulco longitudinali forti; ap. pl. colum. perobliquis, emersis, 2. fortiore, palat. lamellif., 1. introrsum perlonga, perist. crasse labiato, marg. exter. angulatim producto. L. $51 /{ }_{3}$, d. $2 \frac{1}{2} \mathrm{~mm}$. (Algeria). Mal. Alg. 1864 c. f. (B.)

P. lallemantiana B. T. aperturam versus ventricosa, sursum lente conoidea, obsolete costulato-striata, sp. lateribus sat rectis, acuminata; anfr. 9, ult. versus ap. albidus, incrassatus; ap. 2-3-3, angul. magna, par. parva, colum. validis, emersis, palat. lamellif., emersis. I. 7 , d. $3 \mathrm{~mm}$. (Algeria). L. c. 1864 . (B.)

P. lapidaria Hutt. P. secali persimilis, sed anfr. 7 et lam. par. valida; t. fusca, tenue striata, anfr. cylindrici, tres superi celerrime crescentes, apice obtuso, ceteri subæquales, ap. ovato-quadrata, pl. 2-2-4, angul. 1, par. immersa, perist. reflexiusculum, intus nitidum, album, margin. disjunctis. (Afghanistan). Journ. Calcutta 1849. (Hutt.) 
Gen. I o d i c e 1 l a Ad. (Gen. 1855).

Conspectus.

† T. sapiss. ovato- vel cylindraceo-conica, fusco-brunnea, lavigata $v$. irregul. striata; anfr. $7-8$, ult. antice inter sulcos longitudinales planatus, basi obtusc carinatus; ap. infra angulata, marg. colum. verticali, recto, $p l .7-10$, alternantibus, marg. exponsis. (1.)

$+\dagger$ T. sapiss. fusif.-conoidea v. ovatulo-cylindrica, corneobrunnea; anfr. $6-8$, ult. vix planatus nec sulculatus; ap. infra rotundata, pl. 6-9, colum. et palat. suboppositis, marg. subrectis. (2.)

t+† 1'. sapius minor, ovato-conica, oblonga $v$. turvita, tenc attenuata, brunnea, striata ad costulata; anfr. 5-S; ap. plicis nullis $v$. pancis et debitibus.

1. Pyrence.

*) Ap. edentata v. $p l$. 1-4.

**) Ap. pl. 5-7.

2. Sicilia. Ap. edentata v. pl. 1-3.

3. Africa sup.

*) Ap. edentata v. pl. 2.

**) $A p \cdot p l \cdot 4-6$.

4. Europa merid.-orient. et Asia occ.

M. megachilus Jan, T. aperte infundibulif. umbilicata, ovato-conoidea, purpurascenti-fusca; anfr. 7-9, ult. ad sut. tumidus, medio longitudin. impressus, basi compressus; ap. basi subangulata, pl. 9-10: par. 2 (3), profundis, brevibus (exter. cum angul. longa sæpe conjuncta), colum. 2 (1. validiore), palat. 2-5 (1. 2. punctif., 3. 4. 5. elongatis, intus gibbosis), perist. expanso, albo, incrassato, marg. colum, subverticali. L. 10-13, d. $31 / 2-5 \mathrm{~mm}$. (Ttal. super., Helv., Tirol., Gall.). Cat. 1832. Ic. f. 318. - Forma 1) minor, t. rufescenti-cornea, irregul. striata, perist. patulo, incrassatulo, pl. par. externa parva sepius ab angulari disjuncta.

M. goniostonı Kstr. Differt a præcedente præcipue t. rimato-perf., dense capillaceo-striata, anfr. ult. basi ita compresso ut cristato, ap. oblique trigona, basi acute angulata, pl. 2-2-4, angul. longa, sæpe bifida, par. brevi, profunda, palat. 1. minima (sæpe 5. subsuturali), perist. leviter labiato, patulo, marg. exter. infra extenso, colum. stricto, paullo obliquo. L. $9-10$, d. $3 \mathrm{~mm}$. (Pyrencece, Alger.). Mon. 1815 c. f. Ic. f. 939. - Forma 1) juliensis B. (Moll. Loria 1862 c. f.), ap. pl. 2 in angulo dextro (Gall.); 2) aucta W. (Binn. Conch. 1887), ap. pl. palat. 6 (1. breviss., 2. brevi, 3. 4. 5. emersis, 6. basali).

Var. baregiensis (B.) Loc. (Conch. Fr. 1894): t. cylindracea, ventrosula, ap. subtriangularis, pl. 2-1-4, par. parvis 
superne, colum. pone parietem, palat. 1. obsoleta, perist. tenui, acuto; 1. 9, d. $3 \mathrm{~mm}$. Gall., Barìges).

M. angulata Fag. T. perf.-rimata, conico-attenuata, sp. fusif., dense subregul. striata, corneo-luteola; anfr. 12, superi convexi, inferi planulati, ult. basi crista alba valida; ap. obliqua, ovali-compressa, infra angulata, pl. $2-2-5(6)$, angul. longa, palat. 3. emersa, 4.5. punctif., marg. exter. forte incrassato, colum. porcellaneo. L. 11, d. 2 mm. (Hispania). Crón. cient. 1888. Fag.)

M. vasconica Kob. T. fusif.-turrita, subtiliter costulatostriata, rufescenti-cornea, sp. gracili; anfr. 8, convexi, sut. profunda, lenti, ult. ascendens, pone ap. depressus, leviter scrobiculatus, basi compressus; ap. parva, pl. 2-1-3, angul. najore, par. 2. profunda, parva, colum. pone basin, palat. 3. profunda, perist. incrassato, callo par. tenuiss. L. 6, d. $2 \mathrm{~mm}$. (Hisp., Orduna). Jahrb. 1882. (Kob.)

11. bigorriensis (Ch.) Desm. T. aperte umbilicata, cylindraceo-conica, forte attenuata, brunnea, densiss. capillaceostriata; anfr. 7-8, ult. superne convexus, basi rotundatocarinatus; ap. oblongo-ovalis, pl. 2-2-3, angul. perlonga, palat. longis (1. emersa), marg. subæque curvatis, exter. tenue labiato, expansiusculo, colum, reflexo. L. $8-81 / 2$, d. $3-31 / 2 \mathrm{~mm}$. (Pyrencec). Act. Bord. 1835 c. f. Ic. f. 728.

Var, mocquanimun Kstr. (Mon. 1845 c. f.): t. rimatoperf., sursnm lente conico-attenuata, ap. basi distinctius angulata, pl. $2-2-5$, palat. 1. 5. parvis, ceteris validis, perist. simplici; 1.8 , d. $23 / 4 \mathrm{~mm}$.

I. kobelti W. T. oblonga, sp. subconoidea, castanea, ad ap. albida; anfr. 7, ult. lente ascendens, antice planatus, basi rotundatus; ap. trigono-oblonga, pl. $2-1-4$, angul. longa, colum. pone parietem, profunda, antice horizontali, postice forte ascendente, palat. 1. minima, ceteris subæqualibus, intus elatis et parum elongatis, marg. exter. tenui, simplici, supra curvato, deinde strictiusculo, colum. subverticali, crassata. L. $5 \frac{1}{2}$, d. $2 \mathrm{~mm}$. (Hisp., Oviedo). Binn. Conch. 1887.

H. lospitii Fag. T. cylindraceo-conica, corneo-luteola, paullo rufescens, now nitens, striata; anfr. 8, convexi, regulares, ult. vix major, in medio turgidus, infra compressus, multo ascendens; ap. lunato.ovalis, magna, pl. $2-2-3(4)$, ang. tenui, intrante, par. profunda, colum. validis, immersis, palat. 2 superis subæqualibus, 1. submersa, interdum etiam 4. punctif., perist. undique expanso, albo, incrassato, marg. exter. superne valde in curvato, colum. arcuato. L. $7-8$, d. $2 \mathrm{~mm}$. (Hisp. ad Venasque). Crón. cientif. 18s8. (Fag.) 
M. leptochilus Fag. T. subobtecte perf., conica, ventrosula, tenue densiss. striata; anfr. 8, convexi, ult. basi compressus; ap. subrotundata, pl. $2-2-4$, palat. 1. breviss., ceteris callo tenui marginalibus, marg. exter. forte arcuato, tenui, patulo, colum. stricto, reflexo. L. 8 , d. $3 \mathrm{~mm}$. (Gallia merid., Hisp. septemtr.). Soc. Toul. 1879.

Var. elongutissiun Desm. (Descr. 1835 c. f.): t. valde elongata, fusif., substriata, corneo-brunnea, ap. pl. palat. intus evanescentibus, nec gibbis; l. ad $15 \mathrm{~mm}$. (Pyrenææ). Ic. f. 937.

M. crassata (Bof.) Fag. T. subobtecte rimata, fere cylindrica, ad summum sat subito attenuata, corneo-violacea v. rufescens, fere lævis; anfr. 9, rapide regulares, ult. basi compressus, late cristatus, ad ap. descendens; ap. ad sinistram provecta, ovata, pl. $2-2-4$, colum. fere æqualibus, palat. 1 . punctif., ceteris subæqualibus, non emersis, perist. undique expanso, forte incrassato, porcellaneo, marg. colum. obliquo recto, exter. arcuato. L. 10, d. $2^{1}$ mm. (Hisp.). Crón. cient. 1888. (Fag.)

M. schista W. T. cylindraceo-conica, cerasina v. rufescenti-coma, lævigata $\nabla$. irregul. striatula, apice subtransversali; anfr. $7-8$, sat convexi, duo præult. subæquales, ult. non ascendens, antice inter sulculos longos planatus, basi obtuse cristatus, sut. ad ap. obliqua; ap. ovalis, pl. $2-2-4$, angul. perlonga, profunde intus abbreviata, par. compressa, colum. fortibus, horizontalibus, palat. 1. parva, immersa, ceteris longis, subemersis, medio gibbosis, sæpe interruptis; perist. peranguste expanso, rufescente, marg. exter. superne forte curvato. L. $5-6 \frac{1}{2}$, d. $2-2^{1 / 2} \mathrm{~mm}$. (Pyrencec). Binn. Conch. 1887.

M. centralis Fag. T. obeso-conica, forte attenuata, brunnea, sat distanter lamelloso-striata; anfr. 7, convexi, ult. ad basin rotundatus; ap. basi rotundata, pl. $2-2-2$, remotis. L. 7 , d. $3 \mathrm{~mm}$. (Pyrencex, Jourdes). Hist. Pyr. 1892. (Fag.)

II. calpica W. T. subovato-turrita, fulva, costulata, sæpe pruinosa; anfr. $7 \frac{1}{2}$, regulares, superi subcylindracei, ult. depressus, ascendens, basi compressiusculus, sut. profunda; ap. oblongo-semiovata, pl. 1-2_-3, profundis, altis, albis, colum. 1. valida, 2. minuta, palat. 1. subeınersa, 2. breviore, 3. brevi, perist. tenui, simplici. L. 7, d. $3 \mathrm{~mm}$. (Gitrraltar). Nachr. blatt 1872 .

M. tingitnna Kob. T. fusif.-turrita, costis arcuatis sat distantibus sculpta, cornea, sp. gracili; anfr. 7, regulares, ult. penultimo sat altior, basi obtuse cristato-compressus; ap. ovata, interdum callo par. tenuiss., pl. $1-2-3$, sat validis, raro plicula accidentali pone sut. L. $7-8$, d. $3-3 \frac{1}{2} \mathrm{nmm}$. ( $A l$ geria). Jahrb. 1882. - Forma 1) algesira Kob. 1. c., ap. pl.2-2-2. 
I. avenacea Brug. T. fusif--conica, irregul. tenue striata, brunneo-cornea, sæpe pruinosa; anfr. 7-8, sat convexi; ap. oblonga, pl. typice $2-2-3$, remotis, angul. longa v. longiss., palat. brevibus, medio giblosis, perist. subrecto $\nabla$. expansiusculo, tenui, marg. exter. superne angulato-curvato. L. 7, d. $2-21 / 2$ mm. (Furopa, (aucasus). Encycl. meth. 1792. lc. f. 36, 319. Forma 1) cerealis (Z.), t. major, l. 8, d. $21 / 2-3 \mathrm{~mm}$; 2) enpora W., t. typica, ap. lam. par. 2-3, pl. colum. 2, palat. 4 (1. punctif., 4. brevi; 3) paucidens IV. t. typica, sed pl. palat. tantum 2.

Var. subcerennd W. (Fauna suec. 1873): ap. pl. 2-2-4, angulari maxima, palat. subacualibus, remotis; $1.71 / 2-8$, d. $2^{1 / 2} \mathrm{~mm}$. (Suec., Tirol., Tauria).

Var. Permuginen IV. Fauna eur. 1876): t. perf., gracilis, ovato-conica, rufoferruginea, ad sut. distinctius striata, costulis tenuibus paucis distantibus albirlis, anfr. convexi, ult. basi convexiusculus, ap. pl. 3-2-4, pl. par. exter. minima, profundiss., infra angularem, palat. 1. exigua, profunda, mediis longis, intus altis; 1. 4-5, d. 11/2 -2 mm. (Gallia, Agen).

Var. sublaordenu W. (Binn. Conch. 1887): t. minor, anfr. typicis, lævigata, ap. pl. 2-2-2; 1. 5, d. $2 \mathrm{~mm}$.

Var. praknte Iss. (Moll. Pisa 1866): t. fuscobrunnea, irregul. striata, anfr. convexi, sut. profunda, ap. basi circulari, marg. exter. regul. curvato, pl. 2-2-4, palat. 1. minutiss.; I. $5^{1}{ }_{2}$, d. $2 \mathrm{~mm}$. (Ital., Gall., Carinthia).

Vur. Arcadica Reinh. (Sitz. Ber. Berl. 1880): t. conica, ap. marg. exter. superne forti curvato, pl. $1-2-2$, angulari nulla, colum. 1. forti, 2. profunda, parva v. deest; ]. 61/2-71/2, d. $2-2^{1 / 2} \mathrm{~mm}$. (Epirus, Dalm.). - Forma 1) tirolica n., ap. pl. 1-2-4; l. $5^{1}, 2$ d. $2 \frac{1}{2} \mathrm{~mm}$. (Tirolia, Vallarsa).

Var. clienta W. Jahrb. 1883): t. gracile fusif., regul. dense striatula, ap. pl. 2-2-1, palat. 1.4. punctif., mediis longis, 3. emersa, marg. exter. regul. arcuato; 1.7, d. $2 \mathrm{~mm}$. (Galicia, m. Tatra).

Var. oligadonta Del Prete (Bull. It. 1879 c. f.): t. cylindraceo-conica, cinereo-brunnea, grosse striata, anfr. medio planulati, ap. tantum pl. colum. 1 (superne), ceteris lam. \& pl. obsoletis v. nullis, marg. exter. magis curvato; l. 6, d. $21 / 2 \mathrm{~mm}$. (Ital., m. Apuane).

Var. daplicata Kstr. (Mon. 1845 c. f.): t. minor, tenue denseque striata, gracilior, pallidior, anfr. 7, bene convexi, ap. pl. 3-2-4, lam. par. exter. minima, ab angulari sejuncta, pl. palat. 1. exigua; 1. 5, d. $2 \mathrm{~mm}$. (Gall., Helv. \&c.)

Var. meltanostoun (Paul.) W. (Binn. Conch. 1887): t. fusco-castanea, pruinosa, srepius breve et obeso-ovata, sp. ovato-conica (raro conico-elongata), anfr. $61 / 2-7$, convexi, pl. $2-2-3(2)$, perist. tenui; 1. $5-6$, d. $2-2^{1} / 2 \quad\left(4^{1} / 2: 11 / 2\right)$ mm. ('Triest). 
Var. Iepta IV. (Binn. Conch. 1887): t. fusif. turrita, gracile elongata, acuminata, castanea $v$. corneo-brunnea, sublavis, ap. oblonga, pl. 2-2-3, palat. subæqualibus, marg. exter. fortius curvato, recto, tenui; 1.5 , d. $2 \mathrm{~mm}$. (Carniol., Tirol.)

Var. Iransiens WT. l. c.: t. major, solida, cylindraceofusif., sublavigata, rufobrunnea, pruinosa, sp. elongata, anfr. $8-8^{1} / 2$, ap. oblonga, marg. fere aque convexiusculis, pl. 3-2-4; 1. $7^{1} / 2-9^{1 / 2}$, d. $2^{3} / 4-31 / 4 \mathrm{~mm}$. (Tirolia, Ital. super.)

Var. aluundans W. (Nachr. blatt 1893): t. subturritoattenuata, acuminata, rufescens, obsolete striata v. sublævis, anfr. 8, præsertim superi convexi, ap. oblonga, marg. exter. superne curvato, colum. longo, stricto, verticali, pl. 2-2-4, et sæpe lam. minutiss. infra angul., pal. 1. 4. minimis; 1. 8, d. $3 \frac{1}{2} \mathrm{~mm}$. (Græcia).

Var. maritiana Loc. (Conch. Fr. 1894): t. subcylindricoelongata, lente attenuata, brunnea, striatula, anfr. 8-9, sat convexi, sut. profunda, ap. ovali-rotundata, pl. 2-2-3, par. 1. profundiss., colum. subæqualibus, profundis, palat. una alterave marginali; 1. 1.0, d. $3 \mathrm{~mm}$. (Gall. merid.)

Var. aureacensis Loc. l. c.: t. longa, gracilis, cylindrica, brunnea, irlegul. sat forti striata, anfr. 10, subplani, sut. lata, subcanaliculata, ap. parva, sat anguste ovalis, basi retusa, pl. $2-2-3$; l. $9-10$, d. $2 \mathrm{~mm}$. (Gall.)

M. circumplicata (Mss.) W. T. conico-turrita, lævigata, purpureo-fusca; anfr. 8-9, convexi, ult. supra medium canali longitudinali bipartitus; ap. pl. $2-2-3$, angul. et palat. 1. longiss., perist. de cetero pliculus crebris, albis, brevibus circumdato. L. $6 \frac{1}{2}$, d. $2 \frac{1}{2} \mathrm{~mm}$. (Ttal. super.) Fauna eur. 1876. Forma 1) multidentuta Strob. (Ess. 1857), t. minor, sulco in anfr. ult. leviore, perist. intus obsolete crenulato.

M. cereana (Mlilf.) Kstr. T. aperte perf., conico-fusif., subcylindrica, substriata, purpurascenti-fusca; anfr. 7-8, ult. basi rotundato-compressiusculus; ap. oblonga v. ovalis, infra angustata, pl. concoloribus $2-2-4$, angul. mediocri, palat. 1 . obsoleta, reliquis longis, emersis, colum. 2, dentif. L. 7, d. $2{ }^{2} / 3 \mathrm{~mm}$. (Carinth., Gall. merid., Ilisp. bor.) Mon. 1845 c. f.

M. bergomensis (Ch.) Kstr. T. umbilicata, suboratoconica, cornea, costulato-striata; anfr. $7 \frac{1}{2}$, convexi, ult. basi subcompressus; ap. ovalis, basi rotundata, pl. 3-2-3, angul. longa, par. exter. minima, profundiss, colum. profundis, sat fortibus, perist. recto, subsimplici, marg. exter. superne subregul. arcuato, colum. verticali. L. $5 \frac{1 / 2}{2}-6$, d. $21 / 3 \mathrm{~mm}$. (Ital. super.) Mon. 1845 c. f.

Var. lepids W. (Fauna eur. 1876): t. elongatior, anfr. ult. latior et altior, ap. marg. exter. superne fortiter angulato, ad insertionem marg colum. valde approximato; $1.61 / 2$, d. $2 \frac{1}{2} \mathrm{~mm}$. 
I. arigoi (Rm.) Pfr. T. ovato-turrita, lente acute attenuata, violaceo-brunnea, substriata; anfr. $7-8$, convexi, ult. tumidulus, ascendens, luteolus, sut. valde impressa; ap. rotundato-semiovata, pl. $2-2 \cdots 2$, palato subcalloso, perist. subpatulo, labiato. L. $6^{1 / 2}-8$, d. $3^{1 / 2}$ mm. (Hispania). Ic. f. 936 .

M. Insitanica Rm. T. cylindraceo-conica, peroblique irregul. costulata, brunneo-fusca, sp. attenuata; anfr. 8, convexi, ult. ascendens, argute costulata, antice luteola; ap. subovalis, hepatica, pl. $2-2-4$, palat. pellucentibus, 2. curvata, marginali, perist. patulo, subincrassato, marg. exter. superne angulatocurvato. L. 7, d. $3 \mathrm{~mm}$. (Tusitanict). Mon. Hel. 1848. Ic. f. 935.

M. lertosensis Bof. T. ovato-subconoidea, ad apicem obtuse attenuata, corneo-rufescens, striatnla; anfr. $7-8$, convexi, regulares, ult. turgidulus, vix ascendeus; ap. semiovato-rotundata, pl. $2-2-2(3)$, palat. 1. validiore, marginali, ceteris parum immersis, perist. recto, acuto, intus labiato-fulvescenti, marg. valde approximatis. L. 6 , d. $2 \mathrm{~mm}$. (Ilisp., Tortosa). Bull. Fr. 1886. Bof.)

M. nitida Ant. T. rimata, fusif.-ovata, tenuiss. costulatostriata, nitida, flavida; anfr. 6, convexi; ap. magua, subquadrata, pl. 1-1-2. L. 5, d. $1 \frac{2 / 3}{3}$ mm. (Helvetia: Anton, Austria: Zeleb.). Verz. Kstr. Mon. 1815 c. f. (Kstr.)

II. mühlfeldti Kstr. 'T. perf., ovato-conica, corneocastanea, costulata $\nabla$. costulato-striata; anfr. 7, convexi, ult. basi rotundatus; ap. ovato-rotundata, pl. 2-2-2, omnibus acutis, subæqualibus et remotis, marg. valde approximatis, exter. superne angulato-arcuato, colum. inferne arcuato. L. 4-5, d. $2-2^{1 / 3}$ mm. (Dalm., Serbia, Montenegro). Non. 1845 c. f. Ic. f. 320 .

Var. ventilatoris (Parr.) W. (Mal. Blätt. 1875): t. major, umbilicata, irregul. costulata, castanea, ap. interdum pl. palat. 3. basali et pl. 4. minita superne; $1.6 \frac{2}{3}-7$, d. vix $3 \mathrm{~mm}$. (Dalm.)

M. farinesi Desm. T. fusif.-conica, brunnea, substriata, sp. turrito-conica; anfr. 7, convexi, ult. basi rotundatus; ap. oblongo-ovalis, edentata, perist. recto, acuto, simplici, marg. exter. superne angulatim arcuato, colum. subverticali. L. 6, d. $21 / 2 \mathrm{~mm}$. (Gall. merid., Hisp., Lusitania). Soc. Bord. 1835 c. f. Ic. f. 639. - Forma 1) dentiens M. T., ap. tuberculo angulari prædita; 2) subcarinata B., t. elongatior, anfr. ult. basi obsoleto carinatus, ap. oblonga; 3) obesa B., t. brevis, ventriosa, 1. $5-51 / 2$, d. $3-31 / 2 \mathrm{~mm}$. 
M. speluncr (B.) Loc. T. cylindraceo-elongata, leviter attenuata, fuscobrunnea, dense striatula; anfr. $9-10$, ult. vix major; ap. subrectangularis, subexcentrica, edentata, perist. tenui, acuto. L. 8, d. $3 \mathrm{~mm}$. (Pyrencece). Conch. Fr. 1894. (Loc.)

M. boettgeriana $\mathrm{Cl}$. T. turrita, tenuis, brunnea, regul. striatula; anfr. 10-11, convexi, lentiss. crescentes, ult. basi compressulus, sut. profunda; ap. subquadrata, edentata, perist. expansiusculo, acuto, marg. tenuiss. junctis, exter. leviter depresso. L. $61 / 2$, d. $11 / 2 \mathrm{~mm}$. (Hisp., Jaen). Nal. Blätt. 1882 c. f. $(\mathrm{Cl}$.)

M. microdon W. T. rimato-perf., ovato-conica, brunnea, levis, apice rufo obtusiss., sp. $2 \%$ longiore quam ap.; anfr. $61 / 2$, valde et pulchre convexi, superi regulares, penult. antepenultimo multo major, ult. ascendens, sut. immersa, angusta; ap. oblique oblonga, basi recedens, tantum denticulo 1 conico superne et profunde in col., perist. marg. exter. superne forte curvato pone ad colum. producto. L. 4 , d. $1^{2} / 3 \mathrm{~mm}$. (Hisp., Montserrat). Binn. Conch. 1887.

M. tarraconensis Fag. T. sat aperte perf., regul. a basi conica, corneo-luteola, irregul. striatula; anfr. 8 , subconvexi, lente regulares, ult. major, ascendens, basi rotundatus; ap. verticalis, ovato-lunata, pl. $1-3(2-1-0)$, angul. minore, par. plus minusve elongata $\nabla$. punctif. v. nulla, colum. crassa, dentif., perist. recto, marg. fere parallelis. L. 6, d. $2 \mathrm{~mm}$. (Hispania). Cat. Ess. 1888. (Fag.) Cfr. M. jumill. v. ascend. W.

M. jumillensis (Guirao) Rm. T. minutiss. perf., ovatoturrita, brunnea, obsolete striata; anfr. 6, forte convexi, at superne depressuli, ult. prevalens, ventrosula, paullo ascendens, basi vix compressiusculus, sut. profunda; ap. semiovata, subrotunda, pl. $1-1-0$ (plicula angul. perobliqua tenuiss. \& altera in col. stricta, remotissima), perist. subrecto, tenni, simplici, marg. exter. valde curvato. L. 5, d. $21 / 2 \mathrm{~mm}$. (Hispania). Ic. f. 943. - P. biplicata B. Moll. Loria 1863 c. f.

Var. ascendens, W. (Fauna eur. 1876): t. late perf., anfr. $7 \frac{1}{2}$, ult. basi rotunilatus, superne longe alteque ascendens, ap. infra augustior, pl. ut typus at minimis; I. 6, d. $21 / 2 \mathrm{~mm}$.

M. obliterata (Ch.) Kstr. T. subcylindraceo-turrita, sub lente striatula, fuscopurpurea; anîr. $71 / 2$, planiusculi, celeriter crescentes, ult. basi rotundatus; ap. semiovata, lam. I angulari, debeli, pl. 1 colum. obsoleta et pl. 2 palat. immersis (1. punctif., 2. mediocri), perist. incrassatulo, marg. conniventibus, colum. expanso. L. $74 / 5$, d. $21 / 5 \mathrm{~mm}$. (Tusitania). Mon 1845 c. f. (Kstr.) 
M. saltus Fag. T. subobtecte rimato-perf., cylindricoconica, corneo-luteola, rubescens, capillaceo-striata, sp. a basi regul. attenuata, subulata; anfr. 9-10, lenti, ult. ascendens; ap. ovalis, pl. 1-1-1, angul. dentif., callosa, colum. immersa, palat. parva, immersa, marg. exter. incrassato, arcuato, colum. stricto, supra reflexo. L. 7-8, d. $2 \mathrm{~mm}$. (Hisp.) Moll. Ess. 1888. (Fag.)

II. ignota Fag. T. rimata, cylindraceo-turrita, brunneoviolascens v. fusco-cerasina, tenue striatula; anfr. 8, convexi, ult. paullisper ascendens; ap. oblonga, pl. $1-2-0$, sc. angul. minuto et colum. minimis, immersis, superne, perist. recto, simplici, marg. exter. superne forte curvato, colum. dilatato. L. 7-8, d. $3-31 / 2 \mathrm{~mm}$. (Pyrencece). L. c. 1888. (Fag.) P. jumillensis Pfr. Mon. Hel. 1853, B. l. c. 1863 c. f.

\section{(4.)}

II. massotiana B. T. rimata, ovato-turrita, striatula, corneo-castanea, sp. elongata; anfr. 8, convexi, ult. globosus, basi subcarinatus, sut. valde impressa; ap. subrotundato-oblonga, pl. 2-1-1, parvulis, immersis, perist. subrecto, marg. valde approximatis, exter. valde curvato, colum. stricto. L. $61 / 2$, d. $21 / 4 \mathrm{~mm}$. (Hispania). Rev. zool. 1863 c. f.

Var. penchinatiuns B. l. c.: t. rimato-perf, ap. pl. 2-1-2, angul. minutiss., palat. lamellif.; 1. 6, d. $2 \mathrm{~mm}$.

Var. menustata W. (Binn. Conch. 1887): t. late perf., conica, forte striata, ap. infra angustata, marg. oblique extensis infra approximatis, pl. $2-1-2 ; 1.7$, d. $2^{3} / \mathrm{mm}$. (Hisp., Alcazar).

Vur. confusa IV. l. c.: t. ovato-turrita, tenuiss., deuse et peroblique striatula, fusco-cerasina, anfr. ult. basi rotundatus, ap. pl. 2-2-0, angul. tenui, par. immersa, brevi, forti, colum. supernis, immersis, transversis (2. minima). (Catalonia).

II. aragonica Fag. T. rimata, cylindraceo-conica, elongata, luteo-rubescens, tenuiss. irreg. capillaceo-striata; anfr. 10, subconvexi, rapide regul. crescentes, ult. ascendens, basi compressus (sed non carinatus); ap. obliqua, ovalis, pl. 2-1-2, angul. dentif., colum. parva, obliqua, oblique intuenti tantum conspicua, palat. sat brevibus, remotis, perist. undique expanso, albo, marg. colum. fere recto, exter. longiore arcuato. L. 6-9, d. $2-21 / 2 \mathrm{~mm}$. (Hispania). Moll. Éssera 1888. (Fag.)

M. domicella W. T. ovato-conica, costata, rufo-brunnea; anfr. 8, convexi, regulares, ult. basi vix compressus, sut. profunda; ap. ovalis, pl. $2-2-3$, angul. longa, par. multo breviore, immersa, colum. supernis, transversis, immersis, pl. palat. oppo- 
sitis, his non emersis (1. breviss., sat immersa, 2. 3. intus productis), perist. expansiusculo. L. 7, ґ. $2 \frac{1}{2} \mathrm{~mm}$. (Gallia, Lourdes). Verh. Ver. Wien 1892.

M. pulchella Bof. T. perf.-subrimata, conoidea, fuscorufescens, substriata; anfr. 8, valde convexi, lente regulares, ult. subrectus, longitudini breviter bisulcatus, sut. profunda; ap. subovata, pl. 2-2-2, albidis, palat. sat imınersis, parum productis, perist. recto, simplici, marg. callo junctis, exter. valde curvato, basali et colum. expansiusculis. L. $4^{1 / 2}, \mathrm{~d} .11 / 2 \mathrm{~mm}$. (Hispania). Bull. Fr. 1886. (Bof.)

Var. manotiana (B.) W. (Nachr. blatt 1894): t. umbilicata, oblongo-conica, costulato-striata, fusco-brunnea, pl. 6, profundis, albis, brevibus, altis; 1.6, d. $2 \mathrm{~mm}$.

M. ilendensis Fag. T. rimato-perf., conica, striatula, corneo-luteola, sp. elongato-turrita; anfr. 8, regulares, ult. vix major, basi compressiusculus; ap. obliqua, ovata, pl. $2-2-2$, angul. lamellif., par. regul. arcuata, colum. 1. dentif., 2. remota, palat. æqualibus, non emersis, cum colum. exacte oppositis, perist. recto, ad umbil. reflexo, colum. fere recto. L. 6, d. 2 mm. (Hispania). Moll. Éssera 1888. (Fag.)

M. Sardoa Cantr. T. subumbilicata, ovato-cylindracea, costulato-striata, fusco-cornea; anfr. 7, convexi; ap. semiovata, pl. 1-1-3, palat. inferiore maximo, perist. reflexiusculo. L. $4^{1} / 2$, d. $1^{1 / 2} \mathrm{~mm}$. (Sardinia). Malacol. mediterr. 1840. (Cantr.)

M. rupestris Phil. T. perf, turrita, supra medium angusta et forte contorta, dense costulato-striata v. distanter costulata, fuscocornea; anfr. 5-6, valde convexi, sut. perprofunda, ult. et penult. tumido-ventrosi, plus quam dimidiam testre efficientes; ap. ovalis, elentata, perist. marg. perapproximatis, parum inæqualibus, conniventibus, fere æque leviter curvatis. L. 5, d. $2-2 \frac{1}{2} \mathrm{~mm}$. (Sicilia). En. moll. Sic. 1838 c. f. Ic. f. 637 .

M. refuga W. Præcedenti persimilis, sed t. gracilior, distantius et fortius costulata, costulis obtusis, anfr. ult. non ventrosus, ap. oblongo-ovalis, plica alba valida, trausversa, superne in col. prædita. (Sicil. ad Palermo). Nachr. blatt 1892.

II. homala W. T. rimato-perf, ovato-conica, rufescentibrunnea, sat obsolete obtuse costulata, sp. late conica; anfr. $-5 \%$, convexi, regulares, ult. penultimo paullo major, non ven- 
trosus, paullo ascendens; ap. ovata, edentata, callo par. tonuiss., marg. exter. superne forte curvato. L. 5, d. $2^{1 / 4} \mathrm{~mm}$. (Sicilia, m. Galdo). L. c. 1892.

M. occulta (Parr.) Rm. T. vix rimata, fusif-turrita, cornea, costulato-striata v. costulata, tenera; aufr. 5-6, celeriter regulares, penult. antice parum, postice valde convexus, ult. postice tumidus, ad ap. depressus, sut. profunda; ap. ovalis, pl. 1-1-1, profundis, breviss., colum. superua, palat. tenui, subemersa, perist. patulo. L. $4 \frac{1}{2}-5$, d. $13 / 4-2 \mathrm{~mm}$. (Sicilia ad Palermo). Ic. f. 638 .

M. scalaris Ben. T. perf., fusif.-turrita, tenuis, fulvida, costata, sp. gracili et obtusa; anf $3^{\circ} \cdot 6-6 \frac{1}{2}$, ult. convexo-rotundatus, ceteri omnes et præsertim peuultimus subtransversi, medio alti, rotundato-angulati, utrinque compressi et suturas profundas versus declivi, sut. medio canaliculata; ap. ovalioblonga, pl. 1-1-0, par. brevi, immersa, colum. dentif. conica, superne, perist. recto, simplici. L. $4-5$, d. $1-1^{1 / 2} \mathrm{~mm}$. (Sicilia). Ill. sist. cr. \& Nuovo Cat. 1882.

Vur. albidocostata n. t. forte distanter costata, costis albidis; 1. $51 / 2$, d. $21 / 4 \mathrm{~mm}$.

II. unicarinata Pot. \& Mich. T. quam avenacea minor, perf., cylindrica, sursum lente attenuata, cornea, costulata; anfr. 6 , valle convexi, sut. profundiss. et lata; ap. obliqua, rotunda, p1. 2 in columella (2. majore), perist. tenui, expansiusculo, subcontinuo. (Sicilia). Gal. moll. Donai 1838 c. f. (W. in Binn. Conch. 1887 ex Fagot mscr.)

M. dupoteti Terv. M. rupestri similis, sed differt $t$. conica, fuscocornea, pruinosa, tenuiss. striata ac interdum irregul. plicatulo-striata, sp. crassiore, non contorta, anfr. 5, convexis, rapide crescentibus, penult. multo latiore et altiore, sut. immersa, ap. obliqua, elliptica, elentata, marg. distantibus, non conniventibus, exter. valde extra spiram arcuato. L. 4-5, d. $2-2^{1 / 4} \mathrm{~mm}$. (Algeria). Uat. moll. Alg. 1839 c. f.

I. encyphogyra Let. T. aperte perf, conica, elongata, acuminata, nitida, cornea, argute striatula; anfr. 9, tumidoconvexi, ult. Inagnus, ventricosus, sut. profunda; ap. subrotunda, cdentata, marg. valde approximatis, colum. dilatato. L. 4, d. 2 mm. (Algeria). Ann. mal. Fr. 1870. (Let.)

II. brondeli B. T. subperf-rimata, conica, longe attenuata, gracilis, fulvida, eleganter costulata, sp. subulif., acu- 
minata; anfr. 6, convexi, regulares, ult. autice rectus, basi rotundatus; ap. sat obliqua, oblongo-elliptica, pl, 1-1-0, par. minima, colum. forti, horizontali, superua, immersis (sæpius dente punctif, altero extus in pariete). L. 5, d. $2 \mathrm{~mm}$. (Algeria). Moll. Alg. 1864 c. f.

I. barattei L. \& B. T. anguste perf., oblongo-sub. conoidea, inferne potius ventrosa quam attenuata, cornea, striatula; anfr. $5 \frac{1}{2}$, rotundati, regulares, ult. convexus, superne rectus, infra rotundatus; ap. subovata, pl. 2-1-1, minutiss., par. punctif., colum. supera, dentif., palat. valde obsoleta, perist. undique patulo, marg. colum. dilatato et expanso. L. 4, d. $2 \mathrm{~mm}$. ('Tunesia). Prodr. mal. Tun. 1887. (B.)

M. michaudi Terv. T. rimato-perf., fusif.-turrita, fulvida, dense et tenue costulato-striata, sp. gracilif. elongata, acuminata, interdum leviter curvata, superne tenuis; anfr. $7-8$, regulares, convexi, ult. antice impressus, sulco longitudinali, tenui, sut. mediocri; ap. ovata, angustata, pl. 2-2-2, par. superiore nunc parva, nunc elongata (non angulari), colum. supera valida, transversa, 2. parva, palat. 1. medio, elongata, stricta, 2. breviore, interdum basali, l'aro deficiente. L. $61 / 2$, d. $2 \mathrm{~mm}$. (Algeria). Cat. moll. Afr. 1839 c. f. Ic. f. 640.

M. Kabyliana Let. T. rimato-perf., fusif, dense eleganter costulato-striata, cornea, sp. attenuato-acuminata, apice submamillari; anfr. 7, convexi, regulariter sat celeriter crescentes, penult. maximus, ventrosus, ult. minor, antice descendens; ap. semioblonga, pl. 2-2-2, angul. minore, palat. immersis, elongatis, perist. tenuiss. labiato, marg. colum. stricto, dilatato. L. 5, d. $2^{3} / 4$ mm. (Alger.) Ann. mal. Fr. 1870. (Let.)

M. punica L. \& B. T. semiobtecte rimata, oblonga, supra et infra attenuata, medio ventrosula, corneo-castanea, striatula, apice valido; anfr. $5 \frac{1}{2}$, quasi contorti, rotundati, regulares, ult. parum convexus, superne rectus, infra attenuatus; ap. subovata, pl, $2-2-2$, palat. retusis, margine colum. superne dilatato et expanso. L. 4, d. $1^{1} / 2 \mathrm{~mm}$. (Tiunesia). Prodr. mal. Tun. 1887. (B.)

\section{(8.)}

I. philippi Cantr. T. vix rimata, fere fusif., corneobrunnea, regul. striatula, sp. crassula; anfr. 6, convexi, ult. basi rotundatus; ap. ovalis, pl. $2-1-2$, angul, tenui (raro deficiente), par. pertenui, colum. forti, horizontali, valde contorta (antice sæpo hamif.), superna, (raro dente tuberculif. in 
col.), palat. remotis, brevibus (2. minore, sæpe punctif.). L. $4 \frac{1}{2}$, d. $2 \mathrm{~mm}$. (Ital, Dalm., Montenegro, Grecia, Malta). Mal. medit. 1840. - Forma 1) major, 1. ad 51/2 $\mathrm{mm}$. (Armenia).

Var. exigrua Mss. (Coq. Schl. 1863): t. minor, obscure violaceo-cornea, anfr. 5-51/2, ap. dentibus palat. minutis; 1. $3-3^{1 / 2} \mathrm{~mm}$. (Græcia).

Var. savii Ch. (Fagot mscr.): t. regulariter conica, sp. acutior, anfr. minus convexi, sut. levior. (Ital., Pisa).

II. rhodia Roth. T. anguste perf., fusif--turrita, viride v. flavido-cornea, legul. forte striata, sp. gracili, tenui, apice mamillato; anfr. 6-7, ventrosi, sut. profundiss.; ap. truncatoovata, pl. 1-2-2, par. forti, colum. I valida, horizontali, 2. parva, palat. immersis (1. longiss., subemersa, 2. parva). L. $4^{1 / 2}$, d. $1^{3 / 4}$ mm. (Grcecia, Rhodos, Dalm., Syria). Moll. sp. 1839 c. f. Ic. f. 940 .

Vur. thurica Kessler (Reise n. d. Krim 1860): t. major, plicis palat. subærualibus; 1 ad 5 , d. $2 \frac{1}{10} \mathrm{~mm}$. (Tauria).

\section{Gen. G r a n o p u p a Bttg.}

G. granum Drap. T. lente attenuata; aufr. 7-8, convexi, ult. basi rotundatus; ap. ovalis, pl. $1-2-4$, pal. 1. 2 . brevibus, 3. longiore, 4. parvo, marg. exter. angulum subrectum formante. L. 4-6, d. $1^{1 / 2}-1^{3 / 4} \mathrm{~mm}$. (Europa merid., Caucasus, Asia minor, Algeria). Tabl. moll. 1801. Ic. f. 322, 730. Forma 1) minor, sp. breviore, acutiore, $1.31 / 4$, d. $2 \mathrm{~mm}$. ; 2) tetrodon W., ap. pl. 1-2-1 (Piræus).

Var. diluta W. (Fauna europ. 1876): t. albida v. lutescens, anfr. convexiores, sut. profunda, ult. postice angustior, ap. oblongior, plicæ palat. 3 superæ (etiam 1.2.) elongate, 4. brevis (Gall. ad Grasse).

Var. subulata Biv. (Mon. moll. Palermo 1840): t. brevior, obesior, sp. lateribus convexioribus, anfr. $6-7$, ap. magis rotundata, pl. palat. sæpe 5 (h. c. pl. punctif. immersa pone sut.); 1. 4, d. $1^{2 / 3} \mathrm{~mm}$. (Sicilia). Kstr. Mon. 1845 c. f.

Var. poulseni $W$. (Binu. Conch. 1887/: t. eylindrica, apice conico perobtuso, anfr. 7 , perconvexi, ult. penultimo angustior, postice extense convexus, antice lente alteque ascenlens, ap. pl. 1 (cum angul. minutiss.) -2-4 (brevibus), marg. exter. superne forte curvato, colum. sat extenso; l. 4, d. 11/2 mom. (Sicilia).

\section{Gen. 0 d o n t o c y c 1 a s Schlüter (Verz. 1838).}

0. rossmïssleri (Schm.) Rm. T. flexuoso-rimata, costulato-striata; ap. forte labiata, pl. 2-2-3 (3, basali), marg. fere disjunctis. L. 5 , d. $3 \frac{1}{2}$ mm. (Illyria). Ic. f. 644 . 
0. kokeili Rm. T. infundibulif. umbilicata, subtiliter striatula; ap. leviter labiata, pl. 2-2-2 et undique (præcipue in pariete) pliculis tenuibus minoribus interjectis, marg. callo tenui conjunctis. L. $31 / 2-4$, d. $22 / 3-3 \mathrm{~mm}$. (Carinth., Carniol., Croatia, Dalm.). Ic. f. 335. - Forma 1) mitescens W., ap. pliculis interjectis deficientibus.

Gen. P u p i l l a Leach (Brit. Moll. 1820).

$$
\text { Conspectus. }
$$

† Anfr. ult. sulco basali destitutus.

t† Anfr. ult. antice sulco temi basali munitus.

*) Ap. plicula palat. 1 vel mullu.

**) Ap. pliculis palat. sapiss, 2.

P. muscorum Müll. T. ovoideo-cylindracea, obtusa, solidula, rufo-brunnea, lavigata; anfr. 6-7, angusti, sut. immersa, ult. antice coarctatus, sulco transversali et callo albido forti, basi rotundatus; ap. semicircularis, rufescenti labiata, edentata, marg. exter. superne forte curvato. L. $3-3^{1 / 2}$, d. $1^{2} / 3-2 \mathrm{~mm}$. (Europa, Marocco, Alger., Asia occ. et centr.) Verm. hist. 1774. Ic. f. 37. - Forma 1) unidentata C. Pfr., ap. denticulo obtuso profunde in par.; 2) elongata Cl., t. longior, magis cylindrica, anfr. $7-8,1.3 \frac{1}{2}-4 \frac{1}{2}$, d. $1 \frac{1}{2}-1 \frac{1}{10} \mathrm{~mm}$; 3) marginate C. Pfr., t. brevior, latior, apertius perf.; 4) major, 1. 4-5, d. 111/2 $\mathrm{mm}$. (Gallia: Loc.), l. $4^{1 / 5}$, d. $1^{9} / 10 \mathrm{~mm}$. (Transsylv.: Kim.); 5) minor, l. $2 \frac{1}{2}$, d. $1 \frac{1}{2}$ mm., anfr. $51 / 2$ (Transsylv. : Kim.).

Var. simplex Loc. (Conch. Fr. 1894): t. brevis, obesa, anfr. 5-6, convexi, ap. edentata, marg. approximatis; $1.3-31 / 2$, d. $11 / 2 \mathrm{~mm}$.

Var. pratensis Cl. (Fauna Augsb. 1871): t. major, latior, tenuior, fuscobrunnea, dente par. obsoleto; $1.31 / 2$, d. $1 \% 1 / 10 \mathrm{~mm}$.

Var. bigrunata Rm. (Ic. f. 645): ap. dente obtuso medio parietis et tuberculo sat crasso in medio palati prope ad labium.

Var. Inmdstrimi WV. (Sib. moll. 1876): t. brunnea, nitida, anfr. 7, convexi, ult. antice valde ascendens, callo cervicali angusto, concolori, ap. marg. exter. in pariete profundius affixo; $1.3 \frac{3}{3}$, d. $1^{3} / \mathrm{s}$ m. (Suec. super., Norv., Sibiria).

Var. glis WV. (Nachr. blatt 1893): t. ovato-cylindracea, rnfescens, anfr. $61 / 2$, lenti, sut. marginata, ult. callo albo postice diffuso, ap. forte labiata, pl. palat. alta elongata et in palato pone marg. dentibus 2 granulif. validis munita; $1.3, \mathrm{~d} .17 / \mathrm{sm}$. (Brit., Yorkshire). 
Var. transsilvanica Kim. (Verh, sieb. Ver. 1890): anfr. plani, 6-7, sut. superficiali disjuncti, ult. callo leviore, ap. semper fere omnino edentata; $1.21 / 2-3^{7} / 10, \mathrm{~d} .11 / 2-1 \frac{1}{10} \mathrm{~mm}$. (Transsylv., ubi unica forma hujus sp.)

Var. mhsclaryaus Pal. (Nouv. misc. $1866^{\circ}$ c. f.): t. ventricosu-ovata, cornea, sub lente tenuiss. striatula, sp. conica perbrevi, anfr. ult. inter callum et perist. planatus, basi compressiusculus, postice extense convexus, sut. perprofunda, ap. dente par. minimo et dente fortiore in palato, marg. tenue conjunctis; 1. 3, d. 2 mm. (Gallia ad Montpellier). Pal.

P. eumicra B. T. oblongo-cylindrica, forma fere ut O. doliolum, rotundato-obtusiss., corneo-fulva, fere lævis; anfr. 6 , lente regulares, convexi, ult. vix ascendens; ap. rotundata, edentata, perist. acuto, simplici, subrecto, marg. valde approximatis. L. 3, d. $2 \mathrm{~mm}$. (Helv. ad Habshurg). Rev. 1862 c. f. (B).

P. neumeyeri Kstr. T. ovato-cylindrica, obtusa, opaca, pallide cornea, lævigata; anfr. angusti, planiusculi, ult. callo valido; ap. angusta, sat lateralis, subsemilunata, elabiata, pl. par. obliqua et tuberculo ad basin munita, marg. exter. semicirculari. L. $3 \%$, d. $2 \mathrm{~mm}$. (Dalm. ad Ragusa). Non. 1845 c. f. (Kstr.)

P. aucapitainiana $B$. T. profunde et peranguste perf., oblonga, sæpe ventrosula, tenuis, fragilis, lævis, nitida, pallide v. virescenti-cornea; anfr. 51/2-6, lenti, convexi, ult. superne rectus, basi rotundatus; ap. semiovata, edentata, perist. expansiusculo, alboincrassato, marg. approximatis. L. 3, d. 2 mm. (Algeria, Bona). Mal. Alg. 1864 c. f.

P. cupa Jan. T. cylindracea, paullo orata, obtusa, brunnea, sul lente forti regul. striatula, sericea; anfr. 6-7, perconvexi, cylindracei, angusti, sut. profunde immersa, ult. celeriter ascendens, postice convexus, basi rotundatus, ante callum compressum fortem depresso-planatus; ap. rotundata, rufa v. incarnata, lam. I minima in par. et dente 1 punctif. (raro 2) antice in medio palati. L. 3, d. 11/2 mm. (Bavaria, Tirolia, Helv., Ital. super., Hisp. bor., Galicia in Tatra). Mant. 1832. Kstr. Mon. c. f.

Var. madida Gredl. (Tirols Conch. 1856): t. latius perf., cylindrica, latior, tenuior, apice obtusiore, anfr. convexioribus, ult. callo obsoleto, concolori, ap. dente punctif. antice in palato, perist. simplici, elabiato. (Tirol., Helv., Piemont).

Var. eurigrath $n .: t$, tenuis, brunnea, anfr. ult. sine vestigio calli cervicalis, ap. semirotundata, omnino edentata, perist. tenui, simplici, recto; 1. 3, d. $1^{2} / 3 \mathrm{~mm}$. (Græcia, m. Pindus). 
Var. turcuenia Bttg. (Zool. Jahrb. 1889): t. tenuior, anfr. ult. parum ascendens, ap. marg. minus acutis, aut edentula, aut solum dente par. parvulo instructa; $1.3-3 \frac{1}{2}$, d. $1 \frac{5}{\mathrm{~s}}$ mm. (Transcaspia).

Var. carpathica Kim. (Verh. sieb. Ver. 1890): t. callo cervicali parum distincto, ap. edentata. (Transsylv.).

Var. turkestanica n.: t. magnitudine P. muscorum, late perf., cylindracea, cornea, obsolete striatula, anfr. $61 / 2$, regulares, ult. major, antice penultimo duplo altior, longe ascendens, ad ap. albotumidus (loco calli), basin versus planatus, postice rectiuscule declivis, ap. labiata, lam. 1 par. alta, profunda, dente 1 conif. minimo in col. elata, pl. 1 palat. elongata v. brevi v. tuberculif., marg. distantibus, exter. superne forte subrectangulato-arcuato; 1. 3, d. $12 / 3-2 \mathrm{~mm}$. (Turkestan). Variat omnino edentata v. denticulo par.

P. triplicata Stud. T. cylindracea, apice rotundato, fulva, tenuiss. striatula, tenuis; anfr. $6-7$, convexi, angusti, ult. lente ascendens, cervice convexus, basin versus sulculo antice dilatato, postice tenui longitudinali, demum celeriter recte descendens, callo cervicali forti at angusto; ap. rotundata, tenue labiata, lam. 1 par., dente 1 colum. minimo, pl. 1 palat. submediana, mediocri, crassa, intus cauda tenui interdum sat longa. L. $2 \frac{1}{2}-2^{3} / 4$, d. $1-1^{1} / 2$ mm. (Helv., Tirol., Gall., Hisp., Ttal. super., Germ. occ., Mlyria, Austria, Dalm., Transsylv., Caucasus, Armenia). Knrz. Verz. 1820. Ic. f. 324. - Forma 1) edentata; 2) unidentata, in par.; 3) bidentata, in par. et in palato; tridentata $=$ typica.

Var. striatiswn Gredl. (Tir. Conch. 1856): t. obscura, distinctius striata, ap. lam. parietali et pl. palat. elongata impressionem extus carente, dente colum. deficientev. obsoleto. (Tirolia).

Var. Inxurians Reinh. (Jahrb. 1877 c. f.): t. magis cylindrata, ap. pl. palat. 2, superiore leviore, punctif. (Caucasus, Transcaspia).

Var. inops Reinh. 1. c. c. f.: ap. lam. par. parva, sæpe obsoleta et pl. dentif. in col., pl. palat. omnino deficientibus; 1. $21 / 2-2^{3} / 4$, d. $1 \frac{1}{2} \mathrm{~mm}$. (Caucasus).

Var. subuviformis Bttg. (Jahrb. 1879): t. parva, brevis, obesa, ovalis, ap. 3-dentata; 1. 2, d. fere $1 \frac{1}{2} \mathrm{~mm}$. (Transcauc.)

Var. pyrenaica n.: t. parva, ovali-cylindracea, anfr. $5-5 \frac{1}{2}$, angusti, convexi, uit. ad callum cervicalem argutum convexus, cervice nec depresso nec infra sulculato, basi rotundatus, ap. solum denticulo perobsoleto extus in medio palati infra labium; 1. $2^{1} i_{4}$ d. $1 \frac{1}{2} \mathrm{~mm}$. (Pyrenææ orient.).

Var. bilnaca Kim. (Verh. sieb. Ver. 1890): t. magis ovata, sæpius solidior, fusca, denticulo colum. semper deficiente. (Transsylv.). 
Var. mblorevinta Ul. ap. Cl. (Fauna Oester. 1887): t. obesa, curta, ap. late rotundato obtusa (fere forma dolioli), dense regul. striatula, anfr. ult. infra medium late planatus, sulco longitudinali pertenui, lam. par. sat elongata, col. profunde intus acute producta, rubella, pl. palat. sæepiss. elongata; l. $2 \frac{1}{2}$, d. 11/2 mm. (Moravia ad Brünn).

Var, turdimna (B.) Loc. (Conch. Fr, 1894): t. ovoidcoconica, brevis et obesa, rufescens, eleganter striata, anfr. 6 , convexi, sut. profunda, ap. rotundata, obliqua, typice 3-dentata, sed pl. validioribus; $1.2^{1} / 2, \mathrm{~d}_{1} 1^{2} / 2 \mathrm{~mm}$. (Gall. ad Salino in Jura).

P. lonesta W. T. cylindrica, apice rotundata, dense regul. striatula, fulva v. albida; anfr. $7-71 / 2$, convexi, lenti, ult. lente et valde ascendens, infra medium sulco tenui longitudinali, postice abrupte descendens, basi horizontalis, compressus, antice dilatatus et callo angusto albido; ap. 3-dentata, par. interno, colum. profundo nodif., palat. plicif. pone basin, perist. incrassato, callo extus nodiformi continuo. L. 4, d. $2 \mathrm{~mm}$. (Ttalia). Nachr. blatt 1892.

P. halleriana (Ch.) Jeffr. T. oblongo-cylindrica, obtusa, tenue striat:a, sub lente forti densiss. spir. lineata et granulata, rufescenti-cornea; anfr. 7, paullo convexi, regulares, ult. callo cervicali angusto concolori, ad basin crista perbrevi superne fossula terminata; ap. edentata $v$. denticulis minimis, uno in par. et altero in palato, perist. reflexiusculo. L. $3-31 / 2$, d. $2-21 / 2 \mathrm{~mm}$. (Helv., ex. gr. ad pagum Samadin). Ann. a. Mag. n. h. 1855.

P. diecki Gredl. T. cylindrica, gracilis, apice obtuse conico, dense regul. striatula, cornea, sericina; anfr. $7-7 \%$, convexi, ult, prope ap. callo transverso, ad basin obtuse cristatus; ap. rotundata, elabiata, lam. 1 par. forti, immersa, callo punctif, ad insertionem externum, col. intus profundiss. callo dentif., pl. 2 palat. levibus, inter quas extus sulcula obsoleta, perist. late expanso, planato, continuo, acuto, fragili. L. $3 \frac{1}{2}$, d. 11/3 mm. (Turkestan ad Kulab). Nachr. blatt 1889. (Gr.)

P. armeniaca Iss. T. ovato-cylindracea, obtusa, tenuiss. striata, corneo-lutescens, opaca; anfr. 7, convexiusculi, lente regulares, ult. antice subrectus, basi compressus, cristatus, sut. impressa; ap. parva, denticulo profunde in pariete, perist. aperto, incrassato, marg. subparallelis. L. 3, d. $11 / 2 \mathrm{~mm}$. (Armenia ad Eriwan). Moll. Pers. 1865 c. f. (Iss.)

P. signata Mss. T. rimato-perf,, inflato-cylindracea, sublævigata, pallide cornea; anfr. $7-8$, sat convexi, tres superi celeriter aucti, sequentes subæquales, ult. paullo ascendens, 
rotundatus, antice oblique gibbus, tum paullo constrictus; ap. semicircularis, lam. 1 par. intrante, et pl. 1 palat., sulco externo correspondente (pl. colum. nulla), perist. limbo late expanso, forte intus labiato, marg. remotis lamina callosa junctis, exter. sub-noduloso. L. 4, d. $2 \mathrm{~mm}$. (In alluviis flusn. Araxes). Journ. Conch. 1873 c. f.

Var. cristuta Mts. (Moll. Turkest. 1874): t. cylindricoovata, striatula, pallide brunnea, anfr. ult. valde ascendens, basi carina umbilicali obtusa, prope ap. crista transversali munitus, dein strangulatus, ap. denticulo angul. libero, lam. par. mediana, pl. colum. magna, tenui, pl. 2 palat., 2. longiore, marg. remotis, callo tenui junctis, exter, arcuato, colum. subperpendiculari; 1. $3^{2} / 3$, d. $1 \frac{3}{4} \mathrm{~mm}$. (Turkestan in valle Sarafschan).

Var. cyclostomn W. (Nachr. blatt 1893): Var. cristatæ similis, sed alba, hyalina, dense regul, striata, ap. circularis, perist. lamina pariet. alta continuo, anfr. perconvexi, sut. impressa. (Persia ad Scharud).

Var. debilis W. 1. c.: t. cornea, sut. tenuis, ap. marg. longe disjunctis, omnino absque callo par., dente minutiss. profunde in palato, perist. angustiss. reflexo, anfr. ult. antice valde albido-calloso, extus obsoletiss. impresso, basi leviter cristato. (Caucas, ad Krassnowordsk).

P. interrupta Reinh. Differt a P. signata præcipue t. minore, anfr. $61 / 2$, ap. labio superne in marg. exter. dentif. prominente, tuberculo angulari angulatim conjuncto cum lam. parietali immersa, medio interrupta. L. 3, d. $1^{1 / 2} \mathrm{~mm}$. (Caucasus ad Borghom). Jahrb. 1877 c. f.

\section{Gen. S p h y r a d i u m (Ag.) Ch. (Cat. 1837).}

S. edentulum Drp. T. cylindraceo-ovoidea, corneobrunnea, sublævis; anfr. 7 , fere planulati, primi $3-4$ lentiss., 5. 6. latiores, subæquales, ult. altior et latior. L. $21 / 3-2 \frac{2}{3}$, -d. $11 / 4-12 / 3$ mm. (Furopa, Asia bor., oce. \& centr.) Hist. moll. 1805 c. f. Ic. f. 646 .

Var. пвпмим Bttg. (Jahrb. 1880 c. f.): t. minor, angustior, anfr. $6 ; 1.2^{2} / 5$, d. $1 \frac{1}{5} \mathrm{~mm}$. (Caucas., Turkestan).

Var. turritellum W. (Mal. Blätt. 1875): t. ovato-conica, a basi sursum lente attenuata, obtusa, anfr. $1-5$ lente crescentes, nulli latitudine (altitudine) requales. (Suec., Bavar., Germ., Tirolia).

S. columella G. de Mts. T. cylindrica, gracilior, rufohrunnea, tenue at distincte striata; anfr. $7-71 / 2$, convexi, primi 2 celeriter crescentes, apicem brevem formantes, 3.-6. 
latitudine et longitudine subæquales, ult. veutricosus, altior. L. $21 / 2-3$, d. $1 \mathrm{~mm}$. (Europa media montana et boreulis, Sibiria). Würtemb. Fauna 1830. Ic. f. 731.

S. inornatum Mich. T. exacte cylindrica, elongata, obtusa, subtiliss. striata; anfr. 7-9, couvexiusculi, ult. penultimo non major. L. ad $4^{3} / 4$, d. $1^{7} / 8 \mathrm{~mm}$. (Gallia). Compl. 1831 c. f. Eandem speciem e Finlantia habeo, 1. 4, d. $11 / 2 \mathrm{~mm}$.

S. issericum Let. T. cylindrica, elongata, sublævis, tenuis et fragilis, cornea, apice magno, rotundato; anfr. 7, leviss. convexi, lenti, ult. paullo major, convexus, subtus tumidulus; ap. suboblonga, vix excisa, perist. leviss. expanso. L. 4, d. $2 \mathrm{~mm}$. (Algeria ad Oued-Isser). Ann. mal. 1870. (Let.).

Gen. I s t h In i a Gray (Turton Man. 1840).

Conspectus.

+ Plica palat. desuper intuenti profunde conspicua. (1.)

t+ Plica palat. Ilesuper intucnti inconspicna. (2.)

t+t Plica palat. mulla. (3.)

I. strobeli Gredl. T. tenuiter costulato-striata, corneorufescens; anfr. 5-6, ult. parum inajor, basi rotundatus; ap. rotundata, dent. $1-1-1$, par. lamellif., colum. parvo, dentif., palat. guttif. profunde infra lam. parietalem, sæpe intus in plicam attenuato, perist. expanso, tenue labiato, callo par. nitido, tenui. L. $1^{1 / 2}$, d. $^{2 / 3} \mathrm{~mm}$. (Eur. merid. infra Alpes, Caucasus, Armenia, Algeria). Tirols Conch. 1856, Jahrb. 1877 c. f. - Forma 1) unidentata, ap. tantum dente par. vel palat. præsente; 2) bidentata, ap. dente colum. deficiente.

Var. scharlfi Bttg. (Nachr. blatt 1879): t. major, sublevis, anfr. 7, ap. lam. par. longa, magis emersa, dente colum. parvo et d. basali forti. (Gall. ad Bordeaux).

Var. callicratis Scacchi (Osserv. Zool. 1833): t. magis cylindrica, apice subrotundo, subcostulata, anfr. angustiores, convexiores, ult. multo altius ascendens, sut. profundior, ap. typice 3-dentata, variat bidentata v. raro edentata. (Ital. ad Napoli; Sicilia?).

Var. nodosnria De Stef. (Bull. soc. ital. IX): t. anfr. convexioribus, sut. profunda, perist. magis expanso, fortius albolabiato; 1. 2, d. ${ }^{8 / 10} \mathrm{~mm}$. (Italia). - Forma 1) maruccii De Stef., t. elongatior, anfr. minus convexi, magnitudo paullo major; 2) semii De Stef., t. minor, obesior, magis convexa, ap. edentata, l. $11 / 2$, d. ${ }^{4} / 5 \mathrm{~mm}$. 
I. clanstralis Gredl. T. cylindrica v. fusif.-cylindrica, pallide lutea, dense costulato-striata; anfr. $6-6 \frac{1}{2}$, convexi, perangusti, ult. paullo latior, vix ascendens, ad basin sensim attenuato-angustatus; ap. subrotundato-trigona, dent. $1-1-1$, par. sat forti, ad col. approximata, colum. callosa, palat. profundiss., antice incrassato, perist. concolori, tenui, simplici, marg. disjunctis. L. $13 / 4-21 / 4$, d. vix $1 \mathrm{~mm}$. (Tirol., Ital. super., Gall. merid., Dalm., Triest). Tirols Conch. 1856 c. f. Forma 1) anodus Gredl., ap. edentata.

Var. corcyrensis Bttg. (Jahrb. 1883): t. plerumque major, magis cylindrata, apice distinctius conico, anfr. 7, striis densioribus, diametro maximo in media nec in superiore parte testre; 1. 11/2-2, d. ${ }^{3 / 4}-7 / \mathrm{s} \mathrm{mm}$. (Corfu).

Var. clavella Reinh. (Jahrb. 1877, c. f.): t. clavulata, fortiter et remotius costulata, pallide cornea, anfr. primi celerrime crescentes, 3. latiss., ult. basi attenuatus, ap. altior quam lata, lam. par. distincta, dente colum. calloso, profundo, pl. palat. profunde immersa; 1. $1 \frac{1}{2}$, d. $2 / 3 \mathrm{~mm}$. (Caucasus).

Var. opistolon Reinh. (Sitz, ber. Berlin 1879): t. tenue costulato striata, medio ventricosior, ap. fortius dentata (præcipue dente colum. valido) et dente palat. profundiss. immerso, tantum oblique intuenti conspicua. (Banatus).

Var. shlurnensis Reinh. (Jahrb. 1877 c. f.): t. fusif, nitida, lævigata, cornea, anfr. 3. et 4. subrquales, ult. attenuatus, ap. rotundata, lam. par. intrante, dente colum. valido, profundo, pl. palat. oblonga, profunda; $1.11 / 2-1^{3 / 5}, \mathrm{~d} .1 / 2-3 / 5$ mm. ('Tirol. ad Salurn).

I. costulata Nilss. T, cylindrica, apice breviss., dense costulata, gilva v. fulvido-cinerascens; anfr. $6-7$, convexi, ult. valde ascendens, celerrime dilatatus et penultimo duplo latior, antice transversim albido-callosus, postice forte angustatus; ap. ovata, dent. $1-1-1$, pl. palat. crassa, ovali, profunda (fere sub dentem colum.), perist. intus valde albo-calloso. L. 2, d. vix $1 \mathrm{~mm}$. (Succ., Dan., Germ. bor., Moravia, Caucasus). Ilist. moll. Su. 1822.

Var. allogyra W. (Binn. Conch, 1887): t. minor, sursum latior, anfr. 5, valde convexi, cylindracei, sut. perprofunda, ult. vix ascendens, parum penultimo major, postice elongatocurvatus, perist. valde expansum, intus valde labiatum. (Germ. ad Potsdam).

I. odontostoma W. T. dense regul. striata, pallide fulva; anfr. 6-7, ult. callo cervicali nullo v. obsoleto; ap. denticulo profunde in parieto et tuberculo guttiformi superne intus in palato, perist. tenui. L. $21 / 2$, d. ${ }^{3 / 4} \mathrm{~mm}$. (Norv. ad Kristiania). Mal. Blätt. 1875. 
I. striata Gredl. T. fusif.-cylindrica, subtiliter striata, rufo-brunnea (picea); anfr. 6, convexiusculi, ult. duplo major, valde ascencleus, cervice planulatus, infra angustatus; ap. angusta, fere rotundato-trigona, lam. 1 par. longa et pl. 1 palat. valida, oblonga, profundiss., perist. expanso, marg. exter. impresso, producto. L. $21 / 4$, d. ${ }^{4 / 5} \mathrm{~mm}$. (Tirolia, Bavaria merid.). Tirols Conch. 1856 c. f.

I. uniarmata Kstr. T. fere umbilicata, exacte cylindrica, apice late conica, argute costata, corneo-flava; anfr. 7, perconvexi, ad sut. profundam subtruncati, ult. compressiusculus, basi rotundatus; ap. 1-dentata (in palato), marg. colum. expanso, reflexo, col. callosa. L. 2, d. $3 / 4 \mathrm{~mm}$. (Triest). Dritt. Ber. Bamb. 1856. (Kstr.) - Forma 1) westerlundi Anc. in sc., ap. plica longa profundissima in medio palati (Gallia ad Marseille).

I. monodonta Poll. I. minutissimæ similis, sed t. paullo brevior, densius striata (interstitiis vix latitudine striarum, apud minutiss. latioribus) et ap. dente magno guttiformi profunde in palato. (Ttal. ad Torino). Elenco 1885. (Poll.)

I. minutissima Hn. T. cylindrica, dense regul. tenue striata; anfr. 5-6, sat convexi, ult. ascendens; ap. oblongorotundata, edentata, perist. tenui, acuto, marg. disjunctis. L. 2, d. ${ }^{2} / 3 \mathrm{~mm}$. (Europa, Caucasus). Neue Alpina 1821 c. f. Ic. f. 98. Forma 1) lavinscula Kstr. (Mon. 1845 c. f.), t. sublævis, nitida. (Triest).

I. micula Mss. T. lente attenuato-cylindrica, tenuiss. costulato-striata, fusco-grisea; anfr. 8, primi convexi, ult. paullo ascendens, subinflatus, rotundatus; ap. edentata, perist. subrecto, teuue albolabiato, marg. remotis, marg. colum. vix reflexo. L. 2, d. $1 \mathrm{~mm}$. (Caucasus ad. Mahmouth). Journ. Conch. 1873. (Mss.)

I. loumeti L. \& B. T. curta, cylindrica, ad summum ventricosior quam ad basin, cornea, bene striata, sp. brevi, perobtusa, in tholum rotundata; anfr. 5, convexi, ult. superne rectus, prope ap. leviter planatus, ad basin attenuatus; ap. perobliqua, infra retrocedens, edentula, perist. patulescente, marg. remotis, colum. valido, reflexo. L. $1 \frac{1}{2}, \mathrm{~d} .3 / 4 \mathrm{~mm}$. (Tunesia). Prodr. mal. Tunes. 1887. (L. \& B.)

\section{Gen. L e u c o c h i I us (Mts.) Bttg.}

I. théeli W. T. primo aspectu Carych. minimo simillima; sut. profunda perobliqua, anfr. ult. major, basi rotundatus, antice callo transversali concolori cinctus, ap. dent. $1-2-2$, par. alto, compresso, subrectangulari, medio impresso, colum. 1 . 
transversali, 2. minimo, palat, profundis, brevibus, cum callo adhærentibus. L. $2^{1 / 4}$, d. $1 \frac{1}{4} \mathrm{~mm}$. (Sibiria al Mikôilina, (aucasus ad Poti). Sibir. Moll. 1877.

Gen. Vert i g o Müll. (Verm. hist. 1774).

Conspectus.

1. Subgen. Alaa Jeffi. T. dextrorsa.

† Ap. edentata; ccrvix antice vix impressus, nec callo cinctus; ap. elabiata. (1).

†े Ap. dentata.

*) Cervix anticc callo non cinctus.

a. - Ap. callo palatali nullo (pl. palat.

a) Ap. lam. par. 1 v. o. (2).

B) Ap. lam. par. 2 (una angulari). (3).

b. - Ap. pl. palat. callo antice adha-

**) Cervix antice callo transversali cinctus.

a. - Ap. pl. palat. liberis.

a) Cervix uon impressus callo tenui.(5).

B) Cervix post callum fortem impres. sus. (6).

b. - Ap.pl.palat. antice callo adharentibus.

c) Ap. lam. par. 1. (7).

в) Ap. lam. par. 2. (8).

2. Subgen. Vertilla Moq. Tand. T. sinistrorsa. (9).

Subgen. A I a a Jeffr. (Trans. Lin. Soc. 1830).

V. genesii Gredl. T. rimata, ovata, brunnea, nitida, lævigata; anfr. $4 \frac{1}{2}$, tres ultimi lati, lenti, convexi, penult. subventrosus, ult. ad basin postice constrictus, forte rectiuscule declivis, superne ascendens, sut. profunda; ap. marg. exter. superne forte angulato, infra subrecte obliqua. L. $1 \frac{2}{3}$, d. $1 \mathrm{~mm}$. (Tirolia, Suecia). Tirols Conch. 1856 c. f.

V. dicaa W. T. rimata, cylindraceo-ovata, sub forti lente dense striatula, flavescenti-cornea; anfr. 5, regulares, convexiusculi, ult. postice oblique subplanulatus, forte constrictus, antice utrinque dilatatus, sut. profundiuscula; ap. pariete obliquo, marg. exter. regul. arcuato, colum. dilato, patulo. L. $1 \frac{2}{3}$, d. $11 / 3 \mathrm{~mm}$. (Suecia ad Dalbyö). Syn. moll. Scand. 1897. 
V. colata W. T. subperspective perf., obesa, cylindraceoovata, obtusa, fuscorufa, apice albido, sub lente dense striata; anfr. $5 \% / 2$, convexi, tres ultimi lentissimi, antepenult. præcedente fere duplo altior, ultimi duo subequales, ult. antice et postice fere rque altus, postice regul. convexus, non ascendens, sut. impressa; ap. pariete subhorizontali, marg. rectis, exter. regul. arcuato. L. 2, d. $11 / 2 \mathrm{~mm}$. (Suecia ad Dalbyö). Syn. moll. Scand. 1897.

V. inermis W. T. umbilicato-perf, ovata, tenuiss. striata, cormeo-rufescens, sp. breviss., obtusa; anfr. 5, convexi, ult. penultimo parum brevior ac multo latior, penult. a latere dextro visus sut. perobliqua postice, ult. autem antice duplo latiores, ult. valde ascendens, sut. profunda; ap. sat lateralis, deorsum proclucta, ovalis, perist. leviss. labiato, callo par. tenui lato albo, marg. exter. recto, colum. superne reflexo. L. 2, d. $11 / 3 \mathrm{~mm}$. (Sibiria supra Seliwarinstioje). Sibir. moll. 1877.

V. eggeri Gredl. T. umbilicata, ovato-conica, inæqualiter substriatula, nitida, fuscorufa, sp. valde angustata; anfr. 5, convexi, rapide crescentes, ult. subampliatus, breviter ascendens; ap. ampla, perist. albido-limbatum, marg. exter. medio subangulato, superne arcuato-inverso. L. $2^{1 / 2}$, d. $1^{1 / 2} \mathrm{~mm}$. (Tirolia ad Steinegg.) Nachr. blatt 1890. (Gr.)

V. daliaca W. T. oblongo-cylindrica, rufescenti-cornea, lavis; anfr. 5, primi duo parvi, 2. duplo brevior quam 4., ultimi tres subæquales, ult. angustus; ap. dente conico valido superne in col. L. $1^{7 !}$, d. $1^{1 / 3} \mathrm{~mm}$. (Suecia in prov. Dalsland). Binn. Conch. 1887.

Var. dislecarlica W.: t. cylindrica, brunnea, anfr. 5-6, lente regulares, ultimi 2 subrequales, ult. basi rotundatus, sut. medio obliqua, ad ap. subhorizontalis, ap. dente 1 par. remoto breviss. albo, d. 1 in fauce fere ad basin, nodif., immerso, raro etiam denticulis $1--2$ punctif. in medio palati extus; $1.11 / 2-2$, d. $1 \mathrm{~mm}$. (Suecia in prov. Dalarne). Syn. moll. Scand. 1897.

V. pineticola $W$. T. ovato-cylindracea, lente attenuata, rufobrunnea, nitida, striatula; anfr. 5, convexi, sut. subhorizontali, profunda; ap. pariete forte declivi, inferne dente conico. L. $21 / 3-21 / 2$, d. $11 / 2 \mathrm{~mm}$. (Suecia.) Exp. crit. 1871.

V. krauseana Reinh. T. elongato-ovata, rufobrunnea, sub lente tenue denseque striata; aufr. 5, convexi, tres ultimi latitudine subæquales, ult. non ascendens, sut. profunda; ap. dente 1 levi brevique profunde in pariete (rariss. denticulo minimo in col.), marg. tenue lateque conjunctis, exter. superne 
paullo angulato, basali circulari, colum. subverticali, superne reflexo, introrsum dilatato. L. $2^{1 / 5}$, d. $1^{1 / 5} \mathrm{~mm}$. (Sibir. orient.). Sitz. ber. Berl. 1883.

V. hebraica Tristr. T. oblonga, regul. eleganter striata, apice truncato, obtusiss., nitida, cornea, pellucida; anfr. 6 , tertius ultimo ventricosior, ult. ad ap. forte contractus, sut. profunda; ap. subrotunda, sed infra angustata, dente 1 in par. et 2 in col., perist. continuo. L. $2^{3 / 4}$, d. $1 \frac{1}{2} \mathrm{~mm}$. (Jericho). Proc. zool. soc. 1865. ( $\mathrm{Tr}$.)

V. gemma W. T. cylindrico-oblonga, supra medium lente attenuata, obtusa, castanea, nitida, sublævis; anfr. 6, convexi, tres ultimi lati, superiores angusti, ult. non ascendens, basi dilatatus, gibboso-cristatus; ap. infra angustata, dente 1 palat. nodif. infra et denticulo minutiss. supra medium, marg. exter. superne forte arcuato, angulatim producto, infra rectiusculo, col. recta, verticali, basi incrassata. L. $2^{1 / 2}$, d. $1^{1 / 3} \mathrm{~mm}$. (Suecia ad Dalbyö). Syn. moll. Scand. 1897.

V. oroidea W. T. ventricosa, ovalis, medio lata, densiss. striatula, nitida, corneo-lutea, pellucida, sp. late conica; anfr. 5, sut. superne forte declivi, infra vix ascendente; ap. dentibus 3 minimis: $1-1-1$. L. 2, lat. $1 \frac{1}{2} \mathrm{~mm}$. (Suecia ad Tenhult). Exposé crit. 1871.

V. aretica Wallenb. T. ovata, sublævigata $\nabla$. obsolete striatula, castaneo-rufescens v. fulvo-fusca, nitidula; anfr. 5 $-51 / 2$, convexi, ult. parum ascendens, basi tumido-cristatus, sut. profunda angusta; ap. 2-3-dentata: 1-1-1(0), dent. concoloribus, brevibus, profundis, par. minima, perist. incrassatulo, marg. exter. regul. arcuato, supra medium non producto. L. $2 \frac{1}{2}$, d. $1 \frac{1}{2} \mathrm{~mm}$. (Suec. bor. et media, Norv. bor., Fennia, Germ. in Riesengebirge). Mal. Blätt. 1858 c. f.

Var. extiun IV. (Sibir. Moll. 1877): t. late perf., ovatooblonga, brunneo-cornea, anfr. $5 \frac{1}{2}$, ult. antice callo pertenui, concolori, ap. omnino edentata; $1.2^{3} / 4-3$, d. $1^{3 / 4} \mathrm{~mm}$. (Sibir. ad Baklonowskij, Suec. in prov. Jemtland et Södermanland).

Var. tirolensis Gredl. (Verh. Ges. Wien 1869): t. sat late umbilicata, oblongo-ovata, nitida, pellucida, rufescenticornea, anfr. 5, regulares, superi striati, ult. antice callo levi, ap. oblique cordata, dent. 2 apicibus albis, uno lamellif. in par. et altero conico parvo in col., perist. brunneo, leviter labiato. (Tirolia).

V. tatrica Haz. T. elondato-cylingrica, nitida, pellucida, corneo-lutescens, obtusata; anfr. $5 \frac{1}{2}$, convexi, lentissimi, ult. vix $1 / 3$ longit. totius, vix penultimo latior; ap. semiovata, dente 1 par. acuto lamellif., 1 colum. parvo, 1 palat. (sæpe deficiente), marg. exter. regul. curvato, non impresso. L. 2, d. $1 \mathrm{~mm}$. (Tatra). Jahrb. 1885. (Haz.) 
V. alpestris Ald. T. perf, cylindracea, sub lente eleganter striatula, lutescenti-cornea, nitida; anfr. 5, convexi, sut. sat profunda, medio descendente, infra bene ascendente; ap. dent. 1-1-2, albis (palat. sat longis, approximatis, lamellif., par. alto, conico, rotundato, semper præsente), marg. exter. superne in angulum obtusum curvato et angulatim producto. L. $21 / 4$, d. $1 \frac{1}{5} \mathrm{~mm}$. (Eur. Lor. et media, Siliria). Trans. soenorth. 1830. - Forma 1) mitis W., ap. unidentate $(1-0-0$, bidentete $(1-1-0)$ vel tridentata $(1-1-1) ; 2)$ septemtrionatis $W$., t. hand parum major (ut V. arctica), fortins striata, dent. palat. brevibus, concoloribus. (Suecia bor. et med.): 3) elongate Sterki, t. major, cylindrica, brunnescens, lævigata. (Helv. ad Trous .

Var. shuttleworthisuse Ch. ap. Pfr. (Mon. Hel. 1848): t. subovata, sursum lente attenuata, striatula, corneo-brunnea, nitidula, anfr. convexiusculi, sut. tenuis, infra vix ascendens, ap. dent. concoloribus, palat. brevibus, sape minimis (punctif.); l. $1^{2 / 3}$, d. $1 \mathrm{~mm}$. (Helv., Suecia, Dania).

* V. acheila Serv. T, perf., ovata, ad sinistrum convexior, cornea, nitidiss., diaphana, lævigata, obtusa; anfr. 5, lenti, ult. convexus, circa perforationem angulatus, superne recto; ap. trigonalis, infra angulata, transversim recte incrassata, pl. $1-1-2$, par. valida, palat. approximatis (1. validiore, marginali), perist. labiato, marg. colum. rectiusculo ad dextrum obliquo. L. $13 / 4$, d. $1 \frac{1}{4} \mathrm{~mm}$. (Hisp. ad Zaragoza). Et. moll. Esp. 1880. (Serv.)

* V. heldi Cl. T. cylindraceo-subturrita, irregul. tenuiss. striata, rufobrunnea, nitida; anfr. 6, lenti, sat convexi, tres superi lente attenuati, ceteri subæquales, ult, medio sulco tenui, longitudinali; ap. "arcuto-convexa", dent. $1-1-2(0)$, rufescentibus palat immersis, minimis, sæpe deficientibus, perist. continuo, marg. exter. medio impressulo. L. $2 \frac{1}{2}$, d. $1 \frac{1}{5} \mathrm{~mm}$. (Bavaria, Wrürtemberg). Nachr. blatt 1877, Exk. f. ed. 2 c. f. (Cl.)

* V. haensleri Sterki. T. perf, cylindrica, irregul. striatula, bruunea (superne pallidior), nitida; anfr. 6, lente regulares, superi convexioribus, ult. medio planulatus (non impressus), paullo asceudens; ap. subtriangularis, infra angustata, pl. $0-1-2$, palat. approximatis, parallelis, renotis, perist. simplici, acuto, recto, marg. exter. superue arcuato, deinde oblique rectiuscule descendente. L. $21 / 2$, d. vix 1\%/10 mm. (Helv. ad Bryg). Nachr. blatt 1883 c. f. (St.)

* V. schultzi Phil. T. rimata, subcylindrica, lævis, nitida, rufo.fulvida, sp. lente augustata, obtusa; anfr. $5 \frac{1}{2}$, forte convexi: ap. luuato-rotundata, dent. $1-1-3$, palat. immersis, 3. minimo, perist. expansiusculo, marg. exter. arcuato, colum. patulo, L. $2^{1 / 2}$, d. $1^{1 / 3} \mathrm{~mm}$. (Sicilia ad. Palermo). Zeitschr. f. Mal. 1884. Kstr. Mon. c. f. 
* V. helvetica W. T. rimato-perf., louge cylindrica, apice conico, rufescenti-cornea, tenuiss. striata; anfr. $6 \frac{1}{2}$, convexiusculi, regulares, sat alti, ult. parvus, antice ad basin impressus, infra gibbosus, postice forte declivis; ap. obliqua, anguste trigona, infra retusa, dent. $1-1-3$, par. profundo, colum. mediano, palat. 3. minimo, perist. simplici, marg. exter. superne arcuato, deinde oblique descendente, basali et colum. curvatis. L. $2^{3 / 4}$, d. $11 / 4 \mathrm{~mm}$. (Helvetia). Fauna eur. 1876.

* V. Ioontina Gredl. T. punctif. perf., ovato-cylindrica, apice rotundato, tenuiss. eleganter striata, nitidiss., albidocortuea; anfr. $4 \frac{1}{2}$, convexi, alti, regulares; ap. semiovata, forte excisa, dent. 1-1-3, par. linguif., colum. obtuse conico, palat. 1. 2. plicif., 3. punctif., perist. albo, marg. tenue conjuuctis, exter. medio impresso. L. $1 \frac{1}{2}$, d. $1 \mathrm{~mm}$. (Tirolia). Tirols Conch. 1856 c. f. (Gredl.)

*V. biittneri Siem. T. subrimata, ovata, sat obtusa, eleganter striata; anfr. 4-5; ap. semiovata, dent. 1-0-1, perist. albolabiato. "Long. 0,001, lat. 0,015 m." (Curlandia ad Pervesia). Bull. de St. Petersb. VII. 1849 c. f. "Simillima Pupæe bigranatie Rossm., sed veræ Vertiginis characteribus predita dicitur". (Pfr.)

\section{(3).}

V. thibetica Bens. T. rimato-subperf, oblongo-ovata, sublævis, vitrinea, sp. ovata, obtusa; anfr. 5, convexiusculi, ult. asceudens, sut, impressa; ap. obverso-ovata, lam. I angulari, 1 parietali, dent. 2 colum. et pl. 2 palat. profundis, perist. tenui, expansiusculo, colum. leviter reflexo. L. 2, d. $1 \mathrm{~mm}$. (Thibet ad Tskardo). Ann. a. Mag. 1864. (Bens.)

V. eremia W. T. tumido-ovata, ventrosa, fusca, lævigata, aufr. ultimo antice rugoso-striata; anfr. 5, convexi, penult. ultimo multo major, ultimo extus ventroso paullo brevior; ap. subsemirotundata, dent. $1-1-1$ sat validis, par. compresso, colum. conico, palat. crasso, tuberculif. callo albo forti adhærente, marg. callo cum callo aperturali adhærente conjunctis, exter. supra arcuato, infra rectiusculo. L. 2, d. $11 / 2 \mathrm{~mm}$. (Suecia in prov. Medelpael). Syn. moll. Scand. 1897.

V. decora Gould. T. perf,, cylindrico-oblonga, obtusa, rufescenti-cornea, regul striatula, nitida, semper striga unica (ex callo vetero); anfi. 6, forte convexi, regulares, ult. antice impressioribus 2 longitudinalibus, basi compressulus, postice 
declivis, sut. profunda; ap. semirotundata, pariete subhorizontali, dent. $1-1(0)-2$, colum. parvo, profundo, conico, palat. brevibus, altis, interdum intus cauda tenui, marg. disjunctis, simplicibus, rectis. L. $2 \frac{1}{3}$, d. $11 / 3 \mathrm{~mm}$. (Sibiria, Kamtschatka, ins. Behringi). Proc. Bost. soc. 1847 c. f.

V. ronnebyensis W. T. oblongo-ovata, rufobrunnea, nitidiss., dense striatula; anfr. $51 / 2$, convexi, penult, tertio $1 / 3$ major, ult. in statu maturo callo pertenui concolori non impresso cinctus, sut. valde obliqua, infra ascendente; ap. dent. $1-1-2$, concoloribus, par. parvo, colum. minimo, acuto, rufo, pariete valde obliquo. L. $2 \frac{1}{2}$, d. $1 \frac{1}{2} \mathrm{~mm}$. (Suecia ad Ronneby, Germania). Exposé crit. 1871. - Variat dente colum. nullo et dent. par. 2 minimis.

V. graëllsiana Serv. T. oblonga, rubello-cornea, nitidiss., lævigata; sp. sat elongata, apice valido; anfr. 5, convexi, lente regulares, ult. exiguus, rotundatus, superne rectus, basi gibboso-angulatus; ap. semiovata $\mathrm{v}$. subtrigonalis, superne ampla, inferne coarctata, dent. 1-1-2, par. lamellif,, colum. valido, palat. æqualibus, perist. labiato, undique reflexo, marg. approximatis, externo sinuato. L. $2 \%$, d. $1 \frac{1}{4} \mathrm{~mm}$. (Hisp. ad Zaragoza et Granada). Et. moll. Esp. 1880. (Serv.)

V. antivertigo Drap. T. ovata, ventricosa, castanea, sublævis; anfr. 5, convexiusculi, ult. sulco angusto et profundo inter callum et aperturam; ap. typice $2-3-2$ (his lamellif., marginalibus), margine exter. supra medium valde impresso et protracto. L. $2-2 / / 2$, d. $1 \frac{1}{2}-1^{3 / 4} \mathrm{~mm}$. (Europa, Caucas., Armen., Turkestan). Tabl. moll. 1801. Ic. f. 647. - Forma 1) secclentuta Mont., ap. dent. $2-2-2 ; 2$ ) vetolentata Stud., ap. dent. $2-3-2$ vel $2-2-4 ; 3$ irregnlaris Poll., ap. dent. $2-2-3 ; 4$ ) padance Poll., ap. dent. $2-1-3 ; 5$ ) cisalpina Poll., ap. dent. $3-2-2 ; 6$ ) ferox W., ap. dent. $4-3-2(3)$ vel $3-2-4(5)$.

Var. seminalum W. (Exposé crit. 1871): t. minima, ovalis, anfr. 4 , ap. तent. $1(2)-2-2 ; 1.1 \frac{1}{2}$, d. $1 \mathrm{~mm}$. (Suecia; Sicil.??)

Y. lilljeborgi W. T. ovata, ventrosula, castanea, obsolete dense striatula; anfr. 5, convexi, ult. sulco lato non profundo inter callum superne tenuem basi gibbosulum rufum et aperturam; ap. dent. $1-1$ (2, inferiore minimo) -2 (profundis, brevibus, altis), marg. exter. non v. vix impresso. L. $2-2 \%$, d. $1 \%-1 \frac{1}{2} \mathrm{~mm}$. (Suecia, Norv., Fennia, Hibernia). Exposé crit. 1871.

Var. merita IV.: anfr. ult. callo tenuiss. rufo v. perobsoleto, sulco post ap. nullo, ap. dent. $1-1-3(2)$, minimis, nodif. (Suec. ad Östersund, Fennia ad Rissalanranta). Syn. 1897. 
Var. globula W.: t. subglobosa, ventrosiss., brunneocastanea, striatula, .anfr. 4, perconvexi, penult. maximus antepenult. triplo major, nltimo major et prasertim latior, ult. antice dilatatus, callo tenui concolori, postice sulculis parvis, ap. dent. 1 (parvo, profundo) -1 (basali) -2 (punctif., distantibus, submarginalibus); 1. 2, d. $2 \mathrm{~mm}$. (Suecia in pr. Södermanland). Syn. 1897.

V. küsteriana W. Maxime V. moulinsianæ similis, sed t. ventricosior, spira multo obtusior, perist. multo latius reflexum, marg. exter. supra medium valde impresso adeoque arcu marginis superiore inulto fortius curvato, ap. dent. 1-1-2 liberis, palat. superiore magno, antice incrassato, emerso, cum marg. adhærente, dente inferiore profundo a margine longe separato. (Wïrtemberg ad Mergentheim). Nal. Blätt. I875.

V. latasteana L. \& B. T. curta, tumido-ventrosa, subovato-sphærica, fragilis, cornea, lævigata, sp. perobtusa; anfi. 5, convexi, celeriter crescentes, ult. penultimo minus ampliatus, antice ascendens, callo cinctus, compressus, scrobiculatus; ap. minutiss., subtrigonalis, dent. 2 (elongatis, sat validis) -1 (robusto) --2 (lamellif., marg. externum attingentibus), col. valde brevi, marg. remotis, exter. in rostellum producto. L. 2, d. $1 \mathrm{~mm}$. (Tunesia). Prodr. mal. Tun. 1887. (L. \& B.)

V. moulinsiana Dup. T. ovata, medio ventricosa (anfr. penult. $1 / 3$ altior quam antepenult.), lævigata, rufocornea $\mathbf{v}$. fulva; anfr. 5, convexi, ult. antice callo valido albo cinctus; ap. dent. 1-1-2 (his brevibus), marg. exter. medio producto et leviter impresso. L. $2{ }^{3 / 4}-3$, d. $1{ }^{3} / 4-2 \mathrm{~mm}$. (Eur. infra Mare balticum sparsin, Caucas. ad Poti). Cat. extram. Gall. 1849. - Ferma 1) octodentata W., ap. dent. 2-2-2 et pliculis 2 inter marg. colum et basalem.

Var. ventrosa Heyn. (Mal. Blätt. 1862 c. f.): t. perbreve ovata, forte ventrosa, anfr. 4, ap. dent. 1-2 (interiore parvo) -2 (inferiore parvo) $-2 ; 1.2 \frac{1}{4}$, d. $1 \frac{1}{2} \mathrm{~mm}$.

Vur. personata M. T. (Hist. moll. 1855): t. magis extensa, ap. dent. $1-2-2$, marg. sat longe disjunctis.

V. pachygastra Jensen. T. conico-ovata, basi ventricosa, sursum attenuata (anfr. penult. tertio duplo latior et

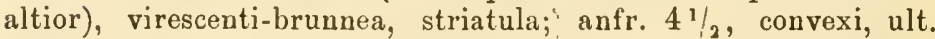
antice callo rufobrunneo; ap. dent. $1-1-2$ (his lamellif.), marg. exter, non impresso. L. $1 \frac{3}{4}$, d. $1 \frac{1}{4} \mathrm{~mm}$. (Norvegia prope Slien). Indberetning 1872. Pupa gravida W. 1873. - Nomen originale hujus speciei in magno genere Pupa inaptum (pro Pupa ejusdem nominis Moq. Tand. 1855 et Rossm. 1837) in genere separato Vertigo restituendum. 
V. pygman Drap. T. ovato-cylindracea, castaneo-1ufa, lævigata; anfr. 6, convexi, sut. infra recta; ap. semiovata, dent. 1 (alto) -2 (sup. conico, inf. punctif., fere basali) -2 (brevibus gibbosis), marg. exter. vix producto, vix impresso. L. $11 / 2-2$, d. $1 \mathrm{~mm}$. (Europa, Caucasus, Asia occ.) Tabl. moll. 1801. Ic. f. 648. - Forma 1) quadridens W., ap. dent. 1-1-2; 2) ausonic De Stef., ap. pl. palat. 1. elongata (Ital.);3) minor, 1. 12/3 $-1^{3 / 4}$, d. $1 \mathrm{~mm}$. (Caucasus).

Var. athesina Gredl. (Tirols Conch, 1856): anfr. ult. pone ap. non impressus, ap. dente 3. supero parvo (Tirol., Ital. sup., Suecia). - Forma 1) bifida W., ap. dente colum. supero conico bifido v. geminato (Suec. ad Dalby).

Var. sarena Gredl. 1. c.: t. major, ap. 8-dentata, sc. colum. 2. in 2 minoribus separato, palat. 4 (1.3. distincte majoribus, 2. 4. obtuse conicis). (Tirolia).

V. laroisiana B. T. ovato-cylindrica, corneo-flavida. nitida, obtusa; anfr. 6, convexi, regulares, sut. impressa; ap. rotundato-lunaris v. obovalis, dent. $1-1-2$, validis, palat. inferioribus, labio interiore adhærentibus, perist. duplici h. e. labio albido et extus margine acuto, tenui, reflexiusculo, discontinuo. L. $2 \frac{1}{2}$, d. $1 \mathrm{~mm}$. (Gallia ad Vannes). Moll. Bret. 1860 c. f. (B. \& Loc.)

V. aprica B. T. rimata, ventroso-oblonga, nitida, rufoAlavido-cornea, tenue striatula, sp. late conica, apice rotundato; anfr. 6, convexi, regulares, ult. vix major, paullo attenuatus, paullo ascendens, basi compressus, callo cervicali albido; ap. semioblonga, dent. $1-1-2$, palat. 1. parvo marginali, 2. plicif., remoto, perist. acuto, intus albido, incrassato, marg. teuuiss. conjunctis, exter. bisinnato, extus impresso, L. $31 / 2$, d. $2 \mathrm{~mm}$. (Algeria). Mal. Alg. 1864 c. f. (B.)

V. codia B. T. perf., forte ventricosa, oblonga, nitida, sublævis, cornea, sp. brevi, apice rotundato; anfr. $51 / 2-6$, convexi, celeriter crescentes, antepenult. permagnus, tumidoconvexus, ult. elongatus, infra compressus, superne paullo ascendens; ap. trigonalis, infra angustata, dent. $1-1$ (immerso) -2 (1. brevi marginali, 2. plicif., immerso), perist. dilatato, intus albolabiato, marg. sat forte conjunctis, exter. bisinuato, extus impresso. L. $21 / 2$, d. $2 \mathrm{~mm}$. (Algeria). L. c. 1864 c. f. (B.)

V. briobia B. T. rimata, oblonga, lævigata, nitida, cornea, sp. convexa, elongata, obtusa; anfr. 5, convexinsculi, regulares, ult. basi compressiusculus, superne rectus, callo cervicali pallido infra fortiore; ap. parum excisa, oblonga, paullo trigonalis, dent. $1-1-2$ (2. fortiore), perist. recto, acuto, intus albolabioto, marg. conjunctis. L. 2, d. $1 \mathrm{~mm}$. (Algeria). L. c. 1864 c. (B.) 
V. microlena B. T. rimata, oblonga, ventricosa, nitida, lævis, cornea, sp. conica, obtusa; anfr. 5, convexi, sat celeriter accrescentes, ult. vix major, elongatus, post callum albidum infra incrassatum planulatus, superne rectus; ap. semirotundata, dent. 1 (forti, lamellif.) -2 (1. forti, longo, 2. parvo basali) -2 fortibus, perist. expanso, profunde intus albolabiato, marg. conjunctis, exter. nou producto nec sinuato. L. 2, d. 11/4 mm. (Algeria). L. c. 1864 c. f. (B.)

V. sieversi Bttg. T. ovato-turrita, corneo-olivacea, subtiliss. sed distincte striata, obtusa; anfr. 5, convexi, ult. antice callo annulari lato sed parum valido fulvido $\mathrm{v}$. albescente cinctus, extus non $\nabla$. vix impressus; ap. dent. $2-2-2$ (plicif.), perist. pallido, sublabiato, marg. tenue junctis, exter. parum producto, vix impresso. L. $1 \% / 8-21 / 8$, d. $1 \mathrm{~mm}$. (Caucasus, Armenia). Jahrb. 1879 c. f. - Forma 1) punctum Bttg., t. minor, magis ovata, l. $15 / 8$, d. $7 / 8 \mathrm{~mm}$; 2) subulpestris Bttg., ap. dent. 1-2 $-2,1.1 \frac{5}{8}$, d. $3 / 4 \mathrm{~mm}$.

V. substriata Jeffr. T. breviter ovata, corneo-flavida, regul. costulato-striata, sericina; anfr. $4 \% / 2$, convexi, ult. callo fulvido, medio impressus, antice ascendens; ap. dent. $2-2-2$, marg. tenue conjunctis, exter. vix producto, parum impresso. L. $1^{3} / 4-2$, d. $1 \frac{1}{4} \mathrm{~mm}$. (Europa borealis et media). Trans. Linn. soc. 1830.

Var. mitis Bttg. (Jahrb. 1880 c. f.): anfr. ult. fere sine callo annulari, ap. dent. levibus, parietali exteriore semper minima; $1.1 \frac{1}{3}-1^{3} / 4$, d. $1 \mathrm{~mm}$. (Caucasus).

Var. monas IV. (Exposé crit. 1871): t. minutissima, ovalis, fusca, anfr. $4 \frac{1}{2}, 1.1 \frac{1}{2}$, d. $1-1 \frac{1}{4} \mathrm{~mm}$. (Suecia, Norvegia, Würtemberg).

Var. sextsnn Gred]. (Nachr. blatt 1872): t. solidior, magis opaca, brunnea, paullo major, distantius striata, callus cervicalis obsoletiss., ap. dent. palat. levioribus, lente colum. infero nullo. (Tirolia).

V. reneaua Serv. T. ovato-globosa, ventrosa, cornea, nitida, sublævigata, sp. convexo-oblonga, apice valido; anfr. 5 , convexi, lenti, sut. profunda, ult. superne rectus, inferne cristato-tuber'culoso, callo cervicali valido; ap. subtrigonalis, dent. 3 (media lamellif.) -2 (1. crassiore) -2 subæqualibus (1. marginali), perist. intus labiato, marg. remotis, exter. sinuato. L. $2 \frac{1}{2}$, d. $1 \frac{1}{2} \mathrm{~mm}$. (Hisp. ad Zaragoza). Etude moll. Esp. 1880. (Serv.)

V. baudoni Mass. T. ovoideo-globosa, flavescenti rufobrunnea, regulariter distanter costulato-striata; aufr. 5, convexi, 
ult. callo cervicali distincto; ap. semirotundata, basi obtussis., dent. 2 (immersis) -2 (remotis) -3 (1 brevi, 2. 3. emersis), perist. continuo, patulo, incrassatulo. L. 2, d. $1 \mathrm{~mm}$. (Gallia, Pyren. or. ad Tautavel). Moll. Pyrén.-Or, 1872. (Loc.)

V. disclıeilia B. T. ventroso-oblonga, nitida, cornea, sub lente striatula, sp. elongato-conica, obtusa; anfr. 5, convexi, rapide crescentes, ult. parvulus, elongatus, superne rectus, basi leviss. compressus, antice callo valido, albido; ap. semirotundata, dent. 2-2 (3), inferiore basali -2 (emersis, extus impressionibus correspondentibus), apud spec, vetera dente pone insertionem marginis, perist. sat profunde alboincrassato, marg. conjunctis, exter. bisinuato, supra medium producto, impresso. L. $2 \frac{1}{2}$, d. $1 \frac{1}{4}$ mm. (Algeria). Paléont. Alg. 1862 c. f. - Forma 1) unilabiata B., anfr. ult. sine callo cervicali.

V. maresi B. T. globosa v. ventroso-ovalis, nitida, corneo-flavida, sublævis, sp. breviss., late conica, perobtusa; anfr. convexi, rapide crescentes, penult. permagnus, ventroso-inflatus, ult. augustatus, attenuatus, lente ascendens, callo sat forti veuoso; ap. semirotundata, dent. $2-2$ (3), 1. forti, 3. minimo -2 (longis, emersis), perist. subrecto, subtenui, marg. tenue junctis, exter. profunde bisinuato, late angulatim producto. L. 2, d. $11 / 4$ mm. (Algeria). L. c. 1862 c. f.

Subgen. Vertill a Moq. Tand. (Hist. mal. 1855).

(Vertigo Müll. Verm. Hist. 1774).

V. pusilla Müll. T. elongato-ovata, lævigata, corneoflavida; anfr. $5-5^{1} / 2$, convexiusculi, ult. antice callo lato cinctus, basi paullo attenuatus; ap. semiovalis, dent. $2-2$ (4, inferioribus minoribus) -2. L. $2 \frac{1}{2}$, d. $1 \frac{1}{4}-1 \frac{1}{2} \mathrm{~mm}$. (Europa, Caucasus, Asia occ.) Verm. hist. 1774. Ic. f. 649.

* Y. tumida W. T. ventricoso-ovata, obtusa, basi ad dextrum tumida, fusca, striatula; anfr. $4 \frac{1}{2}-5$, convexi, ult. antice pallidus, callo cervicali pertenui, basi compressiusculus; ap. late inæqualiter cordata, dent. 2 (1) -2 (I) -3 . L. $17 / 8$ d. $11 / 2$ mm. (Suec., Norv., Dan.) Mal. Blätt. 1867.

*V. collina W. T. ovata, cornea, striatula, sp. brevi, subconica; anfr. $5 \frac{1}{2}$, planiusculi, ult. medio impressus, basi tumidus; ap. subcordata, tantummodo dent. palat. 2 lamellif. L. 2, d. 1\% mm. (Suecia aul Ronneby). Exposé crit. 1871.

V. clevei W. T. ventricoso-ovata; aufr. $5 \frac{1}{2}$, convexi, penult. infra tumidus, ultimo altitudine æqualis, sed multo 
angustior; ap. truncato-ovata, dent. 1-1-0, marg. exter. medio stricto, obliquo. L. $1 \frac{2}{3}$, d. $1 \mathrm{~mm}$. (Suecia ad Sala). Binn. Conch. 1887.

V. erlandi W. T. circulari-perf., oblongo-conoidea, corneo-brunnea, tenue striatula; anfr. 6 , regulares, convexi, sut. usque ad obliqua, ult. collo nullo, lævis; ap. sinuato-ovalis, pariete perobliquo, lam. 1 par. intrante, dent. 2 colum. conicis, pl. 3 palat. crassiusculis, longis, medio impressis v. abruptis (1. emersa, sulco extus correspondente, 3. basali), marg. exter. multo lougiore arcus duos formante, superiore fortiore, producto. L. 2, d. $1 \mathrm{~mm}$. (Suecia ad Dalbyö). Syn. moll. Scand. 1897.

V. angustior Jeffr. T. ovalis, obtusa, infra angustata, regul. striata, fusca; anfr. $5^{1 / 2}-6$, perobliqui, ult. sulco longitudinali ad ap. porrecto præditus; ap. triangulari-cordata, dent. 1-1-1 (lammellif., longo). L. 17/8 mm. (Europa, Caucasus, Asia occ.). Trans. Linn. soc. 1830 . Ic. f. 650 .

Var. папв Mich. (Complem. 1831 c. f.): ap. dent. par. 2, marg. colum. minus incrassato, dente perobsoleto, dente superiore palat. brevi, infero rudimentali. (Gall, ad Lyon, Ital. bor.)

Var. products W. (Fauna eur. 1876): t. elongatosubovata, castanea, anfr. convexi, nlt. postice rectus, perobliquus, basi albogibbosus, medio sulco profundo subbipartitus, antice productus; 1. 2, d. 11/4 mm. (Su., Borgholm).

Var. gothorum W. (Fauna eur. 1876): t. breve ovalis, sat ventricosa, anfr. $4^{1} / 2-5$, vix convexiusculi, ult. medio impressus, basi parum gibbus, peristoma callo junctum; $1.1^{2 / 3}$, d. $1 \mathrm{~mm}$. (Su., Carlshamn).

V. callista W. T. cylindracea, rufobrunnea, dense striatula; anfr. 6, convexi, medii cylindracei, sut. perobliqua, infra non ascendente sejuncti, penult. maximus, ult. postice forte recteque declivis, medio sulco bipartitus, parte super. angusto, basali brevi, tuberoso; ap. subovalis, intus labio calloso rufo, lam. par. 2 (1. breviore, exteriore, 2. profunde intrante), plica palat. 1, longa, intus forte curvata, col. callosa, marg. exter. ut erlandi. L. $11 / 2$, d. $2 / 3 \mathrm{~mm}$. (Suecia ad Dalbyö). Syn. moll. Scand. 1897.

V. otostoma W. T. cylindrica, fulvo-brunnea, striatula; anfr. $61 / 2-7$, convexi; ap. parva, auriformis, pariete valde obliquo, plicis palat. 2 lamellif. et denticulo infra in palato. L. $2^{2} / 3$, d. $1 \frac{1}{3} \mathrm{~nm}$. (Suecia ad Tenhult in prov. Småland). Exp. crit. 1871. 


\section{Index alphabeticus et synonymicus.}

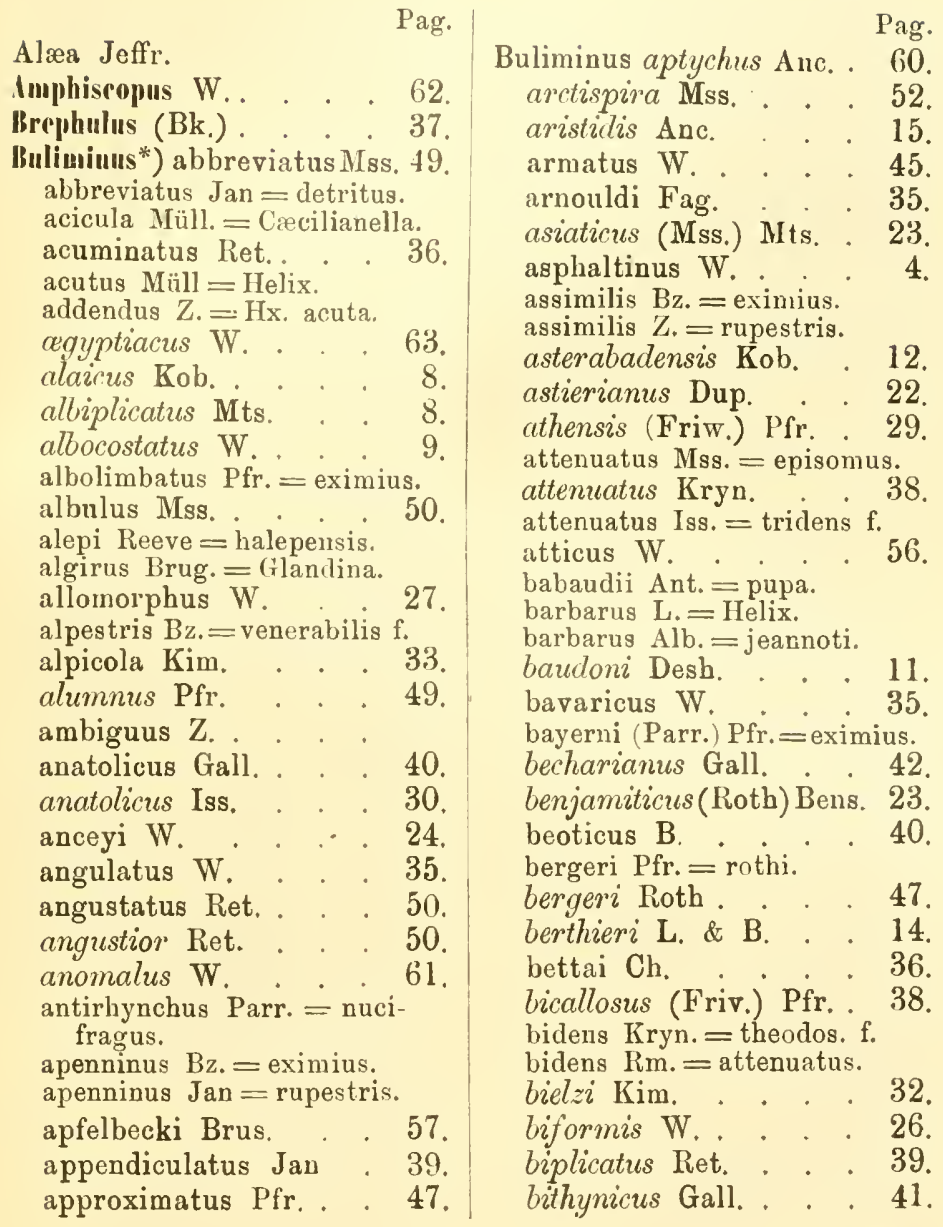

*) Sub hoc nomine generico omnes species et formas palaarcticas ex typo Bulimi stricto huc usque cognitas in hoc indice brevitatis causa junctim enumeravi. 
Pag

Buliminus bituberculatus B. 31 . blandus (Friv.) Pfr. . 46. blanfordianus (Nev.) Kob. 41. blidahensis Kob. . . 16. boettgeri Cl. . . . 21. boettgerianus Kob. . 44. boghariensis Kob. . . 17. bonensis W. . . . 16. bonvallotianus Anc. . 7. borealis Mss. borealis Mss. botterianus Phil. bourguignati Let.

55 . boysianus (Bens.) Reeve bradus Gall.

17.

13.

40. brevior Mss. . . . . 43

bvevis $\mathrm{Bz} .=$ venerab. $\mathrm{f}$. brevis W.

lerondelianus B. 17.

brotianus (Cl.) Kob. . 51

brunnea Bttg. . . 39.

buccinata Alt. (Hx.) $=$ montanus

bulgaricus $\mathrm{B}$.

bulimoides $\mathrm{M}$. $\mathrm{T}^{\circ}=\mathrm{Hx}^{\circ}$. ventrosa.

bulimoides P. (Pupa) $=$ sidoniensis.

cadmoeanus B.

cassius Bttg. .

calcareus Parr. $=$ zebra $f$. callida W. Bttg. . . callosa W. . . . 39. calverti B. . . . . 37. candelaris Pfr. 12 candicans Sh. = kindermanni. candicatus Z. = illibatus. candidus Kstr. (Pnpa) $=$ labrosus.

candidus $\mathrm{Pfr}$.

candisatus Anc.

35.

cantrai Bk. =Cion. pupæformis.

capusianns Anc. carduchus Mts. carneolus (Z.) Mss. carneus (Pfr.) Phil. carpathicus $\mathrm{Cl}$. carpathius Bttg.
Buliminus cartenensis Let. 15. carthusianus Loc. . 21. castaneus W.. . . 10. caucasicus Pfr. . . 21. caucasicus Mss. $=$ eximius. cephalonicus Mss. . . 19. centralis Loc. . . 22. cepistum Mort . . 54. charcius B. . . . 15 . charpentieri Grat. = ovularis. chersonesicus Sow. . 27. chilodius W.. . . 58. chius Mts. . . . 20. chondriformis Mss.. . 55. chondriformis Monter. $=$ pupa $\mathrm{f}$.

chordatus W.

cinereus Mort. $=$ Pupa mortilleti.

cirtanus Morel.

cirtanus in coll. = issericus. clausiliæformis (Fér.)

Mke.

clavatus $\mathrm{Mke}=$ ebuineus. clessini Ret.

codopsis Anc.

collini Mich. $=$ montanus

f. (juv.?)

coloratus Colb. . . . 21 . columellaris W.. . . 22 . compactus (Friv.) Pfr. 40. compositus W. . . 6. concolor W. . . . 19. conemenosi Bttg. . . 19. coniculus Mts. . . . 23. conjunctus $\mathrm{Pfr} .=$ venerabilis. conicus W. . . . 3 . connivens Bttg. . . 21. conoideus Drp. = Helix. consentaneus (Z.) W. . 43. continens Rosen. . 63. controversa Kob.=eremita f. corcyrensis Mss. . . 12 . corneus Mich. . . 34. corneus B. . . . 16 . corneus W. . . . 7. cosensis Reeve $=$ casius. costatus Ret. . . 39. costatus Cl. . . 10. courtieri B. . . . 5 . 
Buliminus cous Mts. 20 crassilabris Rm., Bz. . 33 . crassus Ret. . . . 35. cretensis (Pfr.) Phil. . 29. cruzyi B. . . . 37 cuneolus W. . . . 46 . curta Anc. curtus Bz. = transsylv. $\mathrm{f}$. cymatilis $\mathrm{Ad} .=$ cylindricus.

cylindricus Mke. 36. cylindricoconus Anc. . 24 dalmaticus (Klec.) W. . 46. dardanus (Friw.) Pfr. 35. davidi Desh. . dealbatus (Fér.) $\mathrm{Hx}_{\mathrm{x}}=$ ho . henack. decollatus $\mathrm{L} .=$ Stenogyra. delesserti $\mathrm{B} .=$ subcarneolus. denticulatus Pfr. 58. desgodinsi Anc. destitutus Mss. detritus Müll. dextroversus Anc. didynodus Bttg. difficsus Mss. diminutus Mss. diodon Ret. diplus W. diræ Anc. lirphicus Bl. \& W. discretus W. discolor Bttg. dispistus $\mathrm{B}$. dissimilis Mts. distans W.

$5 \pm$.

26. 22.

29. 59. 20.

distortus W. . . 10.

djurdjurensis Anc. . 14. dominus Bens. . . 13. dorice Iss. . . . . 27. draparnaudi Loc. . 57. drymaus W. dryops Mts. dulines Ret. 60. 19. 54. 39. duboisi Mss. 35. eburneus Pfr. ecallosus W. edentula Risso $=$ olscurus. ehrenbergeri Pfr. elatus Andrz. = eximius. 32.
Buliminus eloicus Rve.

Pag. $=$ bergeri.

elongatus Kob. . , 19. elongatus $\mathrm{Bz}$. . . . 33 . elongatus $\mathrm{Kob} .=$ montanus $\mathrm{f}$. elongatus B. . . 17. elongatus W... . 45 . elongatus (Roth) Mss. . 49. elongatus Requ. . . 57. emarginatus Desh. $=$ pupa. entodon Mts. . . . 60 . episomus B. . . 28. eremita (Bens.) Reeve. 7. errans W. . . 10. etuberculatus Frfld. . 30. etuberculatus W. . . 31 . euboicus Reeve. . 47. eudoxinus Næg. . . 63. euphraticus B. . . 27. euryomphalus (Let.) B. 28. euxinus $\mathrm{Cl}$. . . 52. exacastoma B. . . 5. excellens Ret. . . 59. exilis W. . . . $\mathbf{5 6}$. eximius Rm. . . 45 . exochus B. . . 5. expansilabris W. . 9 . fallax $\mathrm{Z} .=$ montanus. fasciolatus Oliv.. . 35. faux nigra Parr.= fasciolatus.

fedtschenkoi Anc. ferghanensis Kob. flammeus $\mathrm{Z} .=$ bidens. flavescens W. . . 30. fortior W. . . . 24 . fourousi B. . . . 5 . fragilis Parr. $=$ merdue niamus. fraudulentus W. . 49. frivaldskyi Pfr. . . . 38. fuscilabris Kryn. . . 36. fuscostrigatus Bk. . 39. fuscostrigatus W. . 35 . fuscus Friv.-- frivaldskyi. fusiformis Desh. . . 40. fusiformis Mke. . . 36. gaillyi (Let.?) . . 17. galiciensis $\mathrm{Cl}$. 
Buliminus gastrum Ehrbg. 30. gastrum Auct. pl.=pseu. dogastrum.

gaudryi $\mathrm{B} .=$ stylus.

geoffreyi Anc.

germabensis Bttg. germanica Lam. (Pupa) $=$ obtusatus.

ghedeoni Gall. ghilanensis Iss. gibber Kryn. glabratus Mss. godetianus Kob. . . goebeli W.

57. 26. goldfusssi Kob. . . 12. gracilis W. graecus $\mathrm{Bk}$

grandis $\mathrm{Bz} .=$ eximius.

grandis Mss. granulatus W. griffithi (Bens.) guillaini Pini 16. haberhau'ri(Dohrn) Anc. 61 . halepensis Pfr. . . 18. haliciensis (W.). . . 45 . hebraicus $\mathrm{Pfr}$. . . 34. heliconicus B. . . 40. heliconicus B. . . 58 . heptodon Mts. . . 51 . herzi Bttg. . . . . 23. heterostropha Risso = чuadridens.

herzensteini Anc. . . 25. hippolyti Kob. . . . 57. hispalensis W. . . 32 hohenackeri Kryn. . . 35. hoplites W. . . . 52. hordeaceus Brug. =obscurus. humberti B. . . . 22 . illibatus Z. . . . . 36 . imbellis W. . . . 46 . impressus Mss. . . . 55. improbus W. imtuberculus Parr. $=$ pupa. incertus Ret. . inflatus Loc. $=$ tumidus. ingens Bttg. interfuscus Mss = hohenack intermedius Mss.=hohenack. $\mathrm{f}$. internicosta Partsch = duboisi.
Buliminus intumescens Mts. 61 isselianus (B.) Iss. . . 48. issericus Kob. . . . 17 janus W... . . 15. jeanoti Terv. . . . 16. jordani (Boiss.) Ch, . 4 jugurtha Kob.= guillaini. junctus W. kabylianus Let. . . 14. kindermanni (Parr.) Pfr. 29. klecaki W. . . . 46. kolhandensis Mts. . . 8. kollyi Ret. . . . . 47. komarowi Kob. . . 24. komarowi Bttg. . . 59. kordofanus Mts. = hebraicus. kotschyi (Parr.) Pfr. . 6. krueperi Bttg. . . 20. kubanensis Mss. . . 45. kuldschanus (Mss.) Mts. 23. kunawurensis (Hutt.) Reeve . . . 12. kurdistanus (Farr.) Pfr. 35 . kuschakenvitzi Anc. . 8. kutschigi K. . . . . 53. labiatus $Z_{0}=$ pupa.

labiellus Mts.

labrosus Oliv.

lackhamensis Mont. ( $\mathrm{Hx}$.) = montanus.

lævis Blaiuv. = tournefort. lambæsensis Kob. . . 16. lamelliferus $\mathrm{Rm}$. . . 50 . lamprostatus B. . . 5 . langei Bttg. . . . 46. lecouffei Anc. . . . 15. leclereri (Zel.) Pfr. . 62. lederi Bttg. . . . 44. lenomphalus B. . . 37. leptoceras W. . . 10. leptolenus B. . . . 38. letourneuxi B. . . 15. leucodon (Pfr.) Phil. . 43. leucoptychus (Mts.) Anc. 60. levaillantianus B. . . 48. lhotellerii B. . . . 16. libanicus Nrg. . . . 48. limbatus W. . . . 37. 
Buliminus limbatus W. $\quad 37$ limbodentatus Mss.. . 49. limis W. . . . 4 . lineatus Kryn. $=$ cylindricus. liostracus W.

littoralis Brum. $=\mathrm{Hx}_{\mathrm{x}}$ acuta. locardi B. = arnouldi $\mathrm{Fag}$. loewi Phil.

longulus Rolle. . . 11

longulus $\mathrm{Cl}$. . . 60.

lubricus Müll. $=$ Cionella.

lunaticus Jan . . 57

lycicus $\mathrm{Pfr}$. . . 18

major Kob.

malleatus W.

mansurensis Kol. . 14

marcidus Bttg. . 46 .

marginatus $\mathrm{Pfr}$. = guillaini.

marsaliensis W... . 4

martensianus Anc. . 2:3,

mereluenianus Kryn. . 19.

merejkowskii (B.) W. 37.

merloianus Gall. . 42.

mesopotamicrs Mts. 18.

mestus W. . . 31.

meus B. . . . 32.

micans W. . . 54 .

micelï Kob. . . . 18.

michoni (B.) Fag.. . 62.

microstomus Andrz. . 45.

microtragus (Parr.) Rm, 47.

migratus Mil. . . . 45 .

milensis B. . . . 29.

milevianus Raym. . 14.

minima W. . . 45 .

mirus W.. . . . 34 .

misellus W. . . . 26.

miser Mts. . . . . 25.

mintus W. . . . . 6.

montacuti Jeffr. $=$ montanus.

montandoni (Descl.) W. 47.

montanus Drp. . . . 20.

monticola Roth. . . 19.

mosquensis Mil.. . 21.

moupinianus Desh.. . 11.

movradi (Desch.) W. . 55.

munitus W. . . . 53.

mutatus W. . . 47.

mühlfeldi $\mathrm{pfr} .=$ bergeri.

Buliminus myadoniens If Pag

nanus (Ret.) IV. . . 52

narcissei Gall. . . 41

narynensis (Mts.) W. . 8.

neortus W. . . 5

niso Risso . . . 57

nitilissimus Kryn. = ('ionella.

niveres (Parr.) Rm.. . 6

noctivagus P'arr. $=$ subtilis.

nogelli Roth

nueifragus (Parr.) Roth 51 .

nucifragus Mss. = didymodus.

numiclimes B

obesatus $B .=$ episomus.

obeso-acuminatus B.

32.

obesus W.

obesus Loc. $=$ listans.

obesus W.

oblongus (Bl.) Kob. . 35 .

oblongus $\mathrm{W} . \quad . \quad .22$.

obscurus Mïll. . . 22.

obsoletus (Parr.) Mss. 36.

obsoletus Hesse. . 40.

obtusatus Drp. . . . 3.

oidogyra W.. . . 22.

olivaceus (Pfr.) Phil. . 29.

olivetorum Kob. . . 16.

olympicus (Parr.) Kob. 40.

oribatha W. . . . 58 .

orientalis (Friv.) Pfr. . 38.

ossicus Bttg. . . . 19.

ovularis Oliv. . . . 50.

oxianus Mts.. . . 25.

pachychila IV. . , 9 .

parnassicus $\mathrm{W}_{\text {. }}=$ rothi $\mathrm{var}$.

parreyssi Pfr. . . 49.

parvulus Næg. . . , 35.

parvus Bz. . . . 33.

pentodon W. . . 56 .

perdicea (Z.). . . . 39.

perexilis Loc. . . . 22.

perlucens Anc. . . 25 .

perrieri Anc, . . 13.

persicus (Parr.). . 25.

petroplius B. . . 36 .

phasianus (Dub.) Mss. 50 .

phorcus B. . . . 27.

pindieus W. . . . 54 . 
Buliminus piochardi Heynu 35. planicollis W. planilabris Bttg podilica $\mathrm{Cl}=$ secpu.

podolicus (W.) poireti Fér. = Glandina. ponsonbyi W. ponticus Ret.

potaninianus Anc. poupillieri $B=$ numidicus priamus Kob. primitivus (Parr.) W. pringi Pfr. = Helix.

prolixus Parr. proprius W. provectus W. przevaldskii Anc. pseudoepisomus B. pseedogastrum Hesse. pullaster Mts. punicus L. \& B. pupa Brug. pupa $L .=$ detritus. pupæformis Cantr. = Cion. pupoides Kryn. = phasianus. purus W.

\section{pusio Brod.}

quadridens Müll. quadridens Alt. = tridens yuinquedentatus (Mhlf.) quinqueplicatus P. \& M. $=$ quinquedentatus. raddei $\mathbf{K o b}$.

radiatus Brug. . . . 34

rayianus $B$.

raymondi $\mathrm{B} .=$ bicallosus. raynevalianus $B$. reboudi $\mathrm{B}$. 38. regalis Kim. . . . 33 . regelianus Anc. . . 24. regularis Nonter. = pupa $f$. reitteri IIts.

relictus $B z .=$ transsylv. $f$. rembus $\mathrm{B} .=$ obsoletus.

renegatus $\mathrm{W}$. retowskianus $\mathrm{Cl}=$ theodosianus. retrodens Mts. retroplicatus (MIts.) W, 60. reversalis $\mathrm{Bz}$. - venerabilis. revolutus $Z$. - gibber.
Buliminus rimatus Pfr. 10. rivetianus Gall. . . 41. rochebruni Anc. . . 13. rollei Kob. - lycicus. rossmüssleri $\mathrm{Pfr}, \quad . \quad 30$. rothi Pfr. . . . 46. rufistrigatus Bens. . 24. rugosus W. . . . 21 . rugulosus W. . . 50 . rupestris Kryn. . . 19. rupium Da ('. obscurus. sabaudinus (B.) Loc. . 35. saboeanus B. . . . 4. sagax (Friv.) Pfr. . . 55. samarkandensis Kob. . 8 . samavaënsis (Mss.) Pal. 27. samius Mts. . . . 56 . saukyi B. . . . . 54. scapus (Parr.) Pfr. . 58. scharudensis Bttg. . 25. sihleeflii Mss. . . . 28. schmidti $\mathrm{Cl}$. . . 60. secalinus (Mss.) Mts. . 61. seductilis (Z.) Rm. segregatus (Bens.) Reeve 11. semanni Morel. - bourguign. semotus Kim. . . 33. sepium Gmel. Hx. detritus.

septemdentatus Roth . 49. sexdentatus Næg. . . 53. sidoniensis Ch. . . 6. sieversi Mss. . . . 52 . sindicus (Bens.) Rve.. 11. smyruensis W. . . 59 sogdianus Mts. . . 24. solitarius Poir. Hx. unifasc. solivagus $W . . .7$ spirectinus B. . . . . 4. spoliatus (Parr.) Pfr. . 39. spratti (Pfr.) Phil. . 33 spreta $Z$ - eximius.

squalinus $\mathrm{Rm}$. stagnorum Pult.- obscurus. stokesi Bttg. . . 20. strangulatus Loc, . 22. strophostoma Anc. . . 7. sturmi Kstr. . . . 62 stylus (Parr.) Pfr. . . 51. 


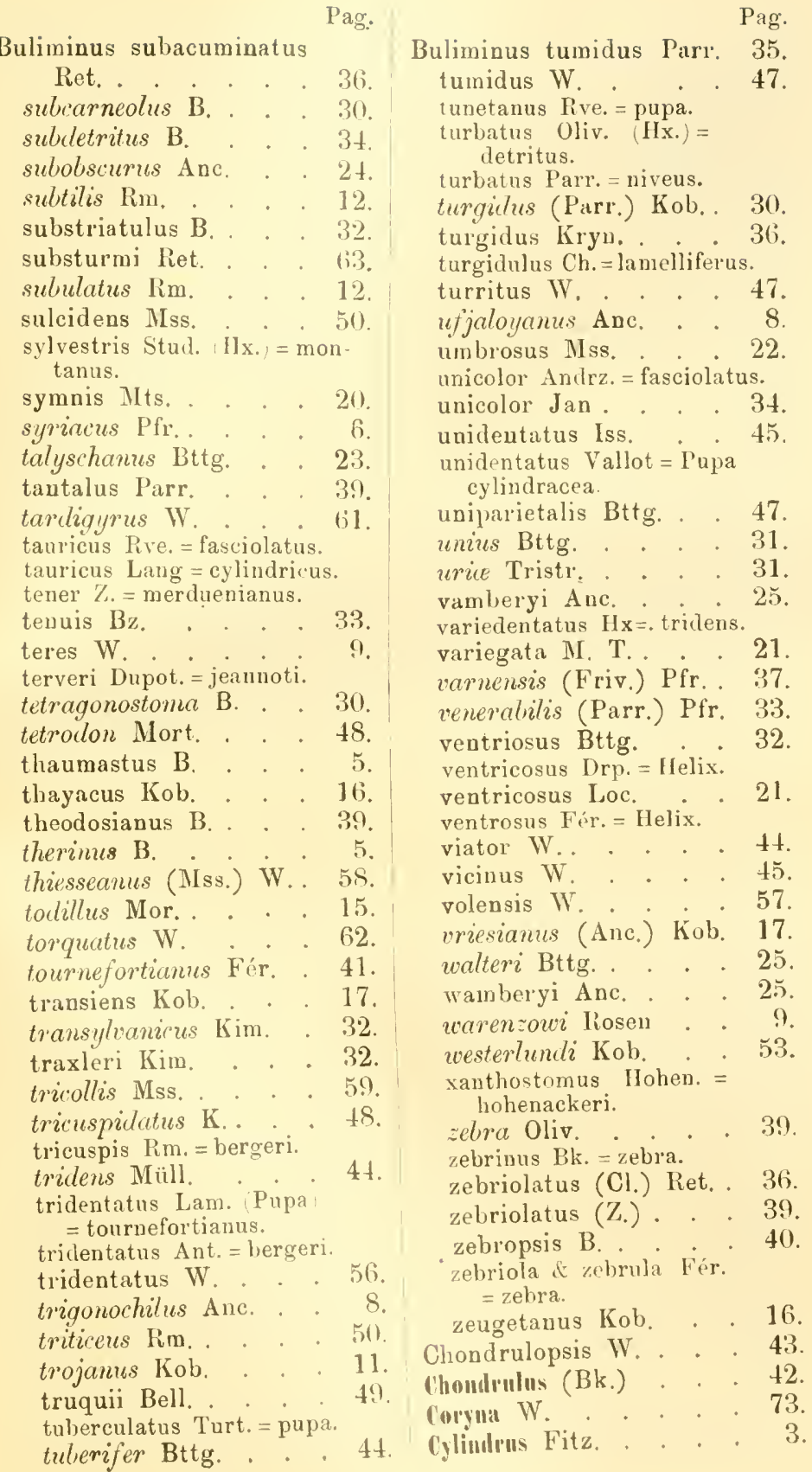




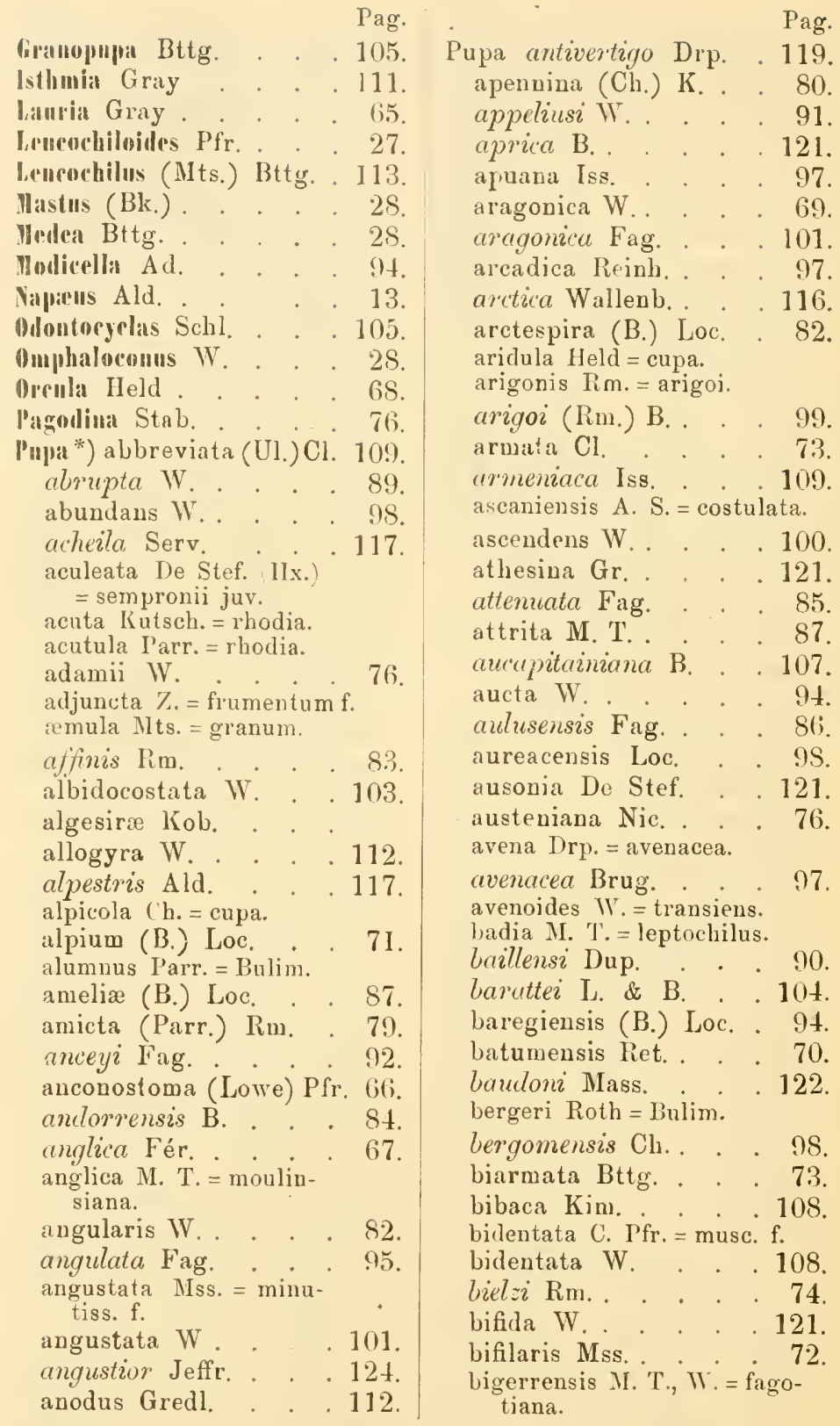

*) In hoc indice omnes formas palæarcticas ex typo Pupæe sub nomine Pupie retuli. 
Pupa bigorriensis (Ch.)

Desm.

bigorriensis $\mathbb{W}^{*}$ = transiens

bigorriensis $\mathrm{Rm}$. = fagotiana

bigranata $\mathrm{Km}$.

106.

bilabiata Ret.

68

bipalatalis W.

93.

biplicata Mich.

biplicata $\mathrm{Rm} .=$ ferrari

biplicata $B$. = jumillensis.

blakei Sh. = cylindracea.

blanci B.

blanci Poll. = limonensis.

boettgeriana $\mathrm{Cl}$. . 100 .

bofilli Fag. . . . 84.

boileausiuna (Ch.) Kstr. 89.

borealis Morel. = decora.

boubeiei $\mathrm{Fag}$. = clansilioides.

bouleausiana Drt. = secale .

bourgeaui Sh. = jumillensis.

bourgetica B. . . 88

bourguignati Cout. . . 76

bourguignati (Macé) . 71.

brancsiki Cl. . . 69.

brauni $\mathrm{Rm} . . .067$.

briobia B. . . . 121

britannica $\mathrm{Ken}$. = Cion. tridens.

brondeli B. . . . 103.

bïtneri Siem. . . . 118.

bugeysiaca Loc. . . 81.

bulimoides $\mathrm{Pfr} .=\mathrm{Bul}$. sidon.

cæsa W.

73.

callicratis Scacchi. . 111 .

callista W.

124

callosa Z. = illyrica

calpica W.

96.

candida Lam. = Bul. labros.

caprearum Phil. = philippi.

carpathica Kim.

108

caspia Pfr.

67.

castanea W. . . . 67

castanea Gr. . . . . 80.

catalonica Bof. . . 84

cancasica Bttg. =inops.

caucasica (Parr.) Pfr. . 68

cebratica W.. . . 69.

cedrorum W. . . 72 .

celata W...... 115.

centralis Fag. . . 96.

cerealis (Z.) W. . . 97.
Pag.

Pupa ierecince (MhIf.) Kstr. 98. charpentieri Sh. $=$ moulins. chrysalis Turt $=$ muscorum. cinerea Drp. = similis. cinerea Mort. = psarolena. circumplicata (Mss.) W. 98. cisalpina Poll. . . . 119. clausitioides Boub. . S6. clausilioides $\mathrm{Pfr}$. = affinis. claustralis Gr. . . 112. clavella Reinh. . . 112. clevei W. . . . . 123. dienta W. . . . 97. colia B. . . . 121. collina $\mathrm{W}$. . . . 123. columella G. de Mts. 110. chondriformis Mss. = Bulim. columnella Loc. . . 92. confusa W. . . . 101 . conica Rm. . . . 70. conospira W.. . . 87. contorta ('alc. $=s p$. dubia. corcyrensis Bttg. . . 112. coronata Stud. $\left(\mathbf{H}_{\mathbf{x}}\right)=$ doliolum.

colrugata Loc. . . 77. costata (Fag.) Loc. . 90 . costulata Nilss. . . 112. costulata Mhlf. $=\mathrm{Hx}$. harpa. (russata (Bof.) Fag. . 96. crassilahris Parr. = Bul. parr.

crassilabrum B. . . 87. crimoda (B.) Loc. . \$ $\$ 1$. cristata Mts. . . 110. cristella W. . . . 88 . critica (Zel.) Pfr. . . 70. cupa Jan . . . . 107. curta M. T. . . . 77. curta $\mathrm{Held}$ = substriata.

curta M. T.

curta Kstr. . . . 8() .

curta Loc. . . . 74 .

curta W. . . . 70.

curta W. . . . . 65.

cyclostoma W. . . 111).

cylindraced Di C. . . 65.

cylindracea W. . . 67.

cylindracea (Z.) Rm.. 80.

cylindrata $\mathrm{Bttg}$. = inops.

cylindrella M. T. = aftinis $\mathrm{f}$. 
Pupa cylindrica Miclı.

cylindrica $\mathrm{Hn}$. = secale.

cylindrica Stud. = avenacea .

cylindrica Loc.

('ylindrica Fér. = minutiss.

cylindriformis (B.) Loc.

dalecarlica W.

daliaca W.

debilis W.

115 .

110.

decemplicata B. . . 88.

decora Gould . . 118.

delphinensis Loc. . . 82.

dentiens M. T. . . 99 .

dentiens M. T. = strobeli f.?

dertosensis Bof.

99.

desmoulinsiana $\mathrm{Ch} .=\mathrm{mou}$ linsiana.

dicoea W. . . . . 114.

diecki Gr. . . . 109.

dilucida (Z) Rm. . 67.

dilucida Wallengr. $=\mathrm{c}$ lindracea $\mathrm{f}$.

diluta W. .

discheilia B

105.

dissimilis $\mathrm{W}$.

90 .

doliformis $\mathbf{L} .=$ doliolum.

doliolum Brug. . . 70.

dolizm Drp.

domicella W.

doumeti L. \& B. . . 11:3.

draparnaudi Leach $=\mathrm{cy}$ -

lindracea.

dufourii Dup. = cylindrica Mich. duodecimcostata Loc. . 88 . duplicata Kstr. . . . 97.

dupoteti Terv. . . . 103.

dupuyi W. = cristella.

ebrodunensis (B.) Loc. 82.

echtinrodes W. . . 77.

edentata W... . . 108 .

edentula M. T. . . 65.

edentula Drp . . . 110 .

edentula Tayl.

edentula Gr. = dilucida.

edentula W.

eggeri $\mathrm{Gr}$.

115.

elongata Rm. . . . 80.

elongata Porro . . 74 .

elongata $\mathrm{Cl}$. . . 106.
Pag.

Pupa elongata M. T. . 90.

elongata Saulcy . . . 88.

elongata Sterki. . . 117.

elongatissima Desm. . 96.

emigrata W. . . 107.

eremia W. . . . 118.

erlandi W. . . . 124.

eucyphogyra Let. . 103.

eudolicha B. . . . 93.

eumicra B. . . . 107.

euodon W. . . 74.

eupora W. . . . 97.

excessiva Gr. . . 75 .

exigua Mss. . . . 105 .

exigua Stud. $\left(\mathrm{H}_{\mathrm{x}}\right.$ ) = edentula.

eximia W. . . . 91 .

extima W. . . . 116.

fagorum (Fag.) Loc. . 89.

fagoti W.. . . . 66.

fagotiana Loc. . . . 90.

farinesi Desm. . . . 99.

farinesii Mich. = leptochilus.

ferox W. .

ferrari Porro . . . 74

ferruginea W. . . 97.

formosa (Parr.) Kstr. . 73.

freseriana Bof. . . 83.

freyeri Sch. = Zospeum.

frumentum Drp. . . 79.

frumentum Boub. = leptoch.

fusiformis Kstr. . . 91.

garumnica Fag. . . 91.

gaudryi B. = Bulim. stylus.

gemma W. . . . 116.

genesii Gr. . . . 114

germanica Lam. Cylindrus.

gigantea M. T. = megachil. f.

glis W. . . . 106.

glaboso-conica W. . 67.

globula W. . . . 120.

goniostoma Kstr. . . 94.

gothorum IV. . . 124.

goodalli Mke. = Cionella.

gourdoniana Fag. . . 89.

gracillimma $\mathrm{Bk} .=$ polyodon $\mathrm{f}$.

gracilis Iss. . . . 66.

gracilis $\mathrm{Rm}$.= bigorriensis.

gracilis Bttg. . . . 76 .

gracilis Haz. . . . 69. 
Pag.

Pag.

Pupa gracilior W. . 88.

Pupa kokeili Rm. . . 106.

graillsiana Serv. . . 119

graniformis Loc. $=$ granum.

gramum Dipp.

105.

gravida $W_{0}=$ Vertigo

pachygast. Jons.

gredleri $\mathrm{Cl} .=$ columella.

gularis Rm.

guuhildæ W. . . 67.

guttula Porro . . 74 .

halleriana (Ch.) Jeffr. 109.

hamata Held = angustior.

hassiaca Pfr. = secale monstr.

hebraica Tristr. . . 116.

heldi $\mathrm{Cl}$. . . . 117.

helvetica W. . . . 118.

heptodonta Risso=sp. inc.

hetrera W.

84.

homala W. . . . 102.

honesta W. . . . 109.

hordeum Stud. $=$ affin. $\mathrm{v}$.

hospitii Fag.

95.

loungarica Kim. . . 80.

haeuslevi Sterki. . 117.

iqnota Fag. . . . 101.

ilendensis Fag. . . . 102.

illyrica Rm. . . . 80 .

implicata Brancs. . 69.

inæqualis W. . . 66 .

inermis W. . . . 115.

inermis W. . . . 65.

inops Reinl.. . . . 108.

inornata Micls. . . 110.

intermedia Kok. = truncatella.

intermedia Ret. . . 72.

intermedia Ret. . . 70.

interrupta Reinl. . . 110.

intrans W. . . . 80.

irregularis Poll.. . . 119.

isarica $\mathrm{W}$. = pygma a fatet. $\mathrm{Cl}$.

ischurostoma (B.) Loc. 82.

isserica Let. . . . 111.

jetschini Kim. . . 70.

juliensis R. . . . 94 .

jemillensis (Guirao) . 100.

jumillensis Rm. = biplicata B.

juniperi Mont. $=$ secale.

kabyliana Let.

104.

kimakowiczi Brancs, = brancs.

kobelti Hid. . . . 95.

kraliki Let. . . . 90.

krauseana Reinh. . . 115.

kriiperi W. . . . 91.

kïsteriana W. . . . 120.

kntschigi Kstr. = Bulim.

labidens Parr. = Bulim.

microtragus.

labiosa M. T. = brauni.

labiosa M. T.

lævigata $\mathrm{Kok}$. = moulinsiana.

lævigata W. . . . 79.

læviuscula Kstr. . . 113.

lallemantiana B. . 93.

larnellata Cl. . . . 74.

lapidaria Hult. . . . 93.

laroisiana B. . . 121.

lasallei (B.) Loc. . . 90.

latasteana L. \& B. . . 120.

ledereri Zel. = Buliminus.

lederi Bttg. . . . . 68.

lederi Bttg. . . . 76.

leontina Gr. . . . 118.

leontina $\mathrm{Cl}$. = isarica.

lepida W. . . . . 98.

lepidula Held = edentula.

lepta W. . . . 99.

leptocheilos Fag. = sequ.

leptochilus (Fag.) W. . 96.

leptospira W. . . . 85̆.

letourneuxi B. . . . 93.

libenotica Tristr. . . 93.

liqustir' Poll. . . . 75.

litietensis Bof. . . . 84.

lilljeborgi W. . . . 119.

limbata Part. $=$ moulins.

limonensis W. . . 82.

lindermeyeri Parr. = scyphus.

locardi (B.) Loc. . $\quad 76$.

longa M. T. . . . 77.

longior Ret. . . . 72.

longurio M. $\mathrm{T}$. = affinis

lucana Brig. = avenacea.

lundströmi W. . . 106.

lusitanica (Rm.) Pfr. . 99.

luxurians Reiuh. . . 105.

macei (B.) Loc. . 71.

macrotriodon (B.) Loc. 71.

madida Gr. . . . 107.

magdalenæ (B.) Loc. . 92. 
Pag.

Pag.

Pupa manotiana (B.) W. 102. maresi B. . - . . 123. marginata C. Pfr. . . 106. marginata L $\mathrm{rp}_{\mathrm{f}}=$ muscol. $\mathrm{f}$. margidens W. . . 75. maritima Loc. . . @S. marruccii De Stef.. . 111. masclaryana Pal. . . 107. masstoiana B. . . . 101. mea (B.) Loc. . . . 81. megachilus Jan . . . 94. megacheilos P. \& N. = leptochilus.

melauostona Paul. . . 97. meledana Stent $z=$ occulta \& rhodia.

menkeana Pfr. $=$ Cion. tridens. merita W. . . . 119. mesopotamica Mss. . 72. michaudi Terv. . . . 104. micheli Dup... . . $9 \mathbf{1}$. michoni B. = Bulim.

microdon W. . . . 100. mirolena B.. . . 122. microtragus Parr. = Bulim.

micula Mss. . . . 113. migma W. . . . 85. minor Rn.

minoricensis $\mathrm{W}=$ porcellata minuta Stud. = sequ.

minutissima Hn. . . 113. misella Paul. . . . $6 \breve{5}$. mitescens W. . . 106. mitis W. . . . . 117. mitis Bttg. . . . 122. modesta $W_{\text {. }}=$ lilljeborgi .

monas W. 122 monodon Held = striata. monodonta Pall. . . 113. montserratica Fag. . 87. montigena W. . . 66. montisicciana Bof. . 84 . moquiniana M. T. = bigorriensis.

moquiniana Kistr. . . 95. mortilleti Stab. . . . 83. mortilleti Bttg. = limonensis. mortilleti Mts, = psarolena. moulinsiana Dup. . . 120. mouliusiana Jeffr., = lilljehorgi. moussoni Reinh. $=$ orientalis.

Pupa mïhljeldti Kstr. . 99. multidentata Auct. mult. = variabilis.

multidentata Strob. . 98. inuscortim Mort. = cylindracea. muscorum L. = avenacea. muscorum Drp. = minutiss. muscorum Müll. . . 106. nana Bttg. . . . 110. nana $P . \& M .=$ mühlfeldti uana Mhlf. = philippi.

nana Mich. . . . 124. nansoutyi Fag. . . . 86. neumeyeri Kistr. . . 107. niciensis Poll. . . 75. nilssoni Wallengr. = anconostoma.

nitens Bttg. . . . 68 . niticla Ant. . . . 99. nitida Mss. $=$ orient. f. nitidula Mss, = sieversi . nodosoria De Stef. . 111. normalis Mke. = Bul. pupa. nova (B.) Loc. . . 81 . novemplicata M. T. . 86. novemplicata B. . . 88. nucifraga Parr. = Bulim. numidica B. . . 67. obesa Parr, = Bulim. albolimb. obesa W. . . . 6?. obesa B. . . . . 99. obesa Blanck. . . 72 . obliqua W. . . . 76 . obliqua Nev.. . . . 82. obliterata (Ch.) Kstr. . 100. oblonga Rm. = pachygastra . obscura Mhlf. = mühlfeldti. obtusa Drp. = Bulim. obtusa Flem. = minutiss. occidentalis (Fag.) W. 86. occulta (Parr.) Rm. . . 103. octodentata Stud. . . 119. octodentata W. . . 120. odontostoma W. . . 112. offtoniensis Sh. = edentula. olearum (B) Loc. . 92. oligodonta Del Prete . 97. olivetorum Loc. . . 79. oparea (B.) Loc. . . 92. opistodon Reinh. . . 112. orrientalis (Parr.) Pfr. 72. 
Pupa oryzana Loc. . . 89.

Pupa psarolena B. . . 79.

otostoma W. . . . 124.

ovoidec IV. . . . 116.

ovularis Oliv. $=$ Bulim.

pulchella Bof. . . . 102.

pulchella M. 'T.

pulchra Ret.

67.

ovulina Loc. . . . 82 .

oyonnaxia Loc. . . . 88.

pachygaster MI. T. = variab. $\mathrm{f}$.

pachygastra Jeus. . . 120.

pachygastra (Z). Rm. 80.

pachygastra W. . 79.

padana Poll.. . . 119.

pagodula Desm. . . 76.

pallicla (Phil.) Rm. . 79.

palustris Leach = antivertigo.

par WV.

69.

parreyssi (Friv.) Pfr. 73. partioti (M. T.) St. Sim. S8. parvula Mss. = triplicata.

patula Mke. = pallida.

paucidens W.

penchinatiana B. . . 101.

perlonga Bof. . . 83.

perplicata Sterki . 76. personata M. T. . 120 .

pfeifferi M. T. = dol. f. mniplicata. petrophila Fag. . . 84. philippii Cantr. . . 104. phthisica Bof. . . . S3. pliynata W. . . . 89. pineticole W. . . . 115. piniana Fag. . . . 89. plagionixa (B.) Loc. . 79. plagiostoma A. Br. . 69. plagiostoma (B.) Loc. 82. plicata A. Müll. = angustior.

polita Risso

polita $W$.

polloneræ Pini = spoliata.

polyodon Drp.

punctum Bttg.

punica L. \& B. . . 104.

pupula Held = minutiss.

pusilla Müll. . . . 123.

pusilla M. T. . . . 90.

pusillima $\mathrm{Z} .=$ minutiss.

pygmea Mab. = laroisiana.

pygmcea Drp.

121

pyrenæaria Pfr. = vergnes.

pyrencearia (Boub.) Mich. 85.

pyrenaica $\mathrm{Bk}$. = pyrenearia .

pyreuaica W. . . . 108.

pyrenaica Boub. $=$ ringens

\& pyrencearia.

pyrenaica Fér. = leptochilus.

quadridens Drp. = Bulim.

quadridens W. . . . 121

quadridentata Stud. = pygm. f.

quadriplicata Loc. . . 69.

quinquedentata Stud. = pygmæa.

quinquedentata Born. = variab.?

quinquedentata Mhlf. = Bulim.

quinquedentata Anct. pl.

= similis.

quinquelamellata Risso

$$
\text { = similis. }
$$

quinqueplicata Mhlf. = mühlfeldti.

raymondi B. . . . 72 .

refuga W. . . . 102.

reneana Serv.

ressmanni Villa = excessiva.

restituta W. . . . 69.

retracta W. . . . $\$ 6$.

rhodanica Loc. . . 81 .

rhodia Roth.. . . 105.

ringens (Caill.) Mich. . 90.

ringens Jeffr. = anglica .

ringicula Mich. . . 87.

rivierana Bens. $=$ strobeli.

romanica Kim. . . 74.

ronnebyensis W. . . 119.

rossmä̈sleri (Schm.) . 105.

rossmässleri Fag. = fagotiana.

rupestris Phil. . . 102.

rustica (B.) Loc. . . 81 .

rusticula (B.) Loc. . . 92.

sabaudina B. . . . 67 . 
Pag.

Pag.

Pupa sabaudina Loc. . 81. sæva W. . . . 83.

saint-simonis B. . . 71.

saliuiensis (B.) Loc.

saltus Fag. . . . 101.

salurnensis Reinh. . 112.

sardoa Cantr.

121.

sarena Gr.

121.

saulcyi B. = granum.

savii Ch.

105.

saxicola M. T.

scalaris Ben. . . 103.

scharffi Bttg. . . 111.

schista W. . . . . 96.

schmidti Kstr. . . 70.

schranki Roth. = striata.

schult zi Phil. . . 117.

scyphus (Eriv.) Pfr. . 71.

secale Drp. . . . . 88.

secale Desm, = leptoch.

seductilis Z. = Bulim. niso.

seligo Roth. . . . 88 .

semidens W. . . 86.

semii De Stef. . . 111.

seminulum W. . . 119.

sempronii Chi . . . 66.

septemdentata Fér. = anti-

vertigo.

septemtrionalis W. . 117.

sexdentata Mont. . . 119.

sexplicata Bof.

sextana Gr. . . . 122.

shuttleworthian Ch. . 117.

shuttleworthiana Gr. \& alior. $=$ alpestris.

sieversi Bttg. . . . 122.

signata Mss. . . . 109.

similis Brug. . . . 79.

simii De Stef. . . . 111.

simoni Bttg. . . . . 83.

simplex Loc. . . . 106.

sinianocoriensis Mss. . 71.

sinuata Mss. = antivertigo.

sitella Kstr.

spelta $\mathrm{Bk}$. = avenacea $\mathrm{f}$.

spelunca (B.) Loc. . . 100.

spinellii Gr.

spinosa Fér. $(\mathrm{Hx}$.$) dolio$

lum juv.

spoliata Rm.. . . 69.

Pupa squalina Rm. = Bulim.

sterri Voith = cupa.

striata Gr. . . . 113.

striatissa Gr. . . . 108.

strobeli Gr. . . . . 111.

sturmi Kistr. = Bulim.

stylus Barr. = Bulim.

subalpestris littg. . . 122.

subcarinata B . . . 99.

subcereana IV. . . . 97.

subdola Gr. . . . 76.

subhordeum W. . . 97.

sublævigata B. . . 88 .

sublævis (B) Loc. . . 71.

suboviformis Bttg. . 108 .

subperforata B. . . 66.

subringens Fag. . . 90 .

substriata Jeffr. . .

subulata Biv. . . 105.

subventricosa Dup. = leptochilus.

sulculata $\operatorname{Riss} 0=$ sp. inc.

superstructa Mss. . . 68.

tardiana (B.) Loc. . . 109.

tatrica W. . . . 116.

taurica Kessler. . 105.

templorum Bin. $\left(H_{x}.\right)=$ doliolum juw.

tenuimarginata Desm. = leptochilus.

tereticollis W. . . 70 .

tetrodon W. . . . 105.

thelii W. . . . 113.

thibetica Bens. . . 119.

tingitana Kob. . . . 96.

tirolensis $\mathrm{Gr}$. . . 116.

tirolica IV. . . . 97.

toscaniæ B. . . . 75 .

transiens $\mathrm{TT}$. . . 98 .

transiens Boub. = pyrena aria \& vergnesiana.

transsilvanica Kim. . 107.

transversulis W. . . 69.

tricolor Villa = megachilus.

tricupis Rm. = Bulim. bergeri .

tridens Drp. = Bulim.

tridens Alt. = frumentum.

tridens Pult. $=$ Cionella.

tridentalis Mich.= triplicata.

tridentata Ant. $=$ Bulim . bergeri. 
Pupa tridentata Lam. $=$ Bulim. tournefort.

tridentata Brard = Bulim. tridens.

tridentata $\mathrm{Rm} .=$ amicta.

trifilaris Mss.

72.

trilamellata $\operatorname{Risso}=\mathrm{sp}$. inc.

triplicata Stud. . . 108.

triplicata Brancs. . 69.

triticum $\%$. = apennina.

truncatella (7.) Pfr. . 73.

truncatella $\mathrm{Rm} .=$ parreyssi.

tschapecki Gr. . . 77.

tumida IV. . . . . 123 .

tumida Haz. . . . 69.

turkestanica IV. . . $10 \mathrm{~S}$.

turcica Let. . . . 71.

turcmeuia Bttg. . . 108.

turgida Parr, = frument. v. curta.

turritella W. . . . 110. umbilicata Drp. = cylindracea.

umbilicus Roth . . 66. uniarmata K. . . 113.

unibasalis Bttg. . 68. unicarinate P. \& M. 103. unidentata Vall. (Bul. = eylindracea.

unidentata C. Pfr. . 106.

unidentata W. . . 108.

unilabiata $\mathrm{IT}$. . . 117.

uniplicata P. \& M. 69.

valcourtiana (B.) Loc. 92.
Pupa vallisnerii De Stef. (Hx.) = sempronii juv.

valsabina Gr. ol. = spinellii.

valsabina Spin. . . 75. variabilis Drp. . . 81 . variabilis C. Pfr. = secale $\mathrm{f}$. variegella Jan. . . 79. vasconica Kob. . . . 95. venetzii Ch. = angustior . ventilatoris (Parr.) W. 99. ventricosa Loc.. .

ventrosa Heyn. . . 120. vergnesiana (Ch.) Kstr. 85. vertigo Gm., Aleron. $=$ pusilla.

vertigo $\mathrm{Hn} .=$ antivertigo. vertigo Iss. = angustior .

villæ Ch. . . . . 66.

villosula $\mathrm{Kok} .\left(\mathrm{Hx}_{\mathrm{x}}\right)=\mathrm{an}-$ gustior juv.

vulcanica Kstr. . . 67.

vulgaris Jeffr. = pygmra.

westerlundi Anc. . . 113.

zonata Bttg.. . . 68.

Pupilla Leach . . . 106.

Retowskia Bttg. . . 28.

Samuhlia W. . . . 77.

Sesteria B. 3.

Sewertzowia Kob. . . 62.

Sphyradium (Ag.) Ch.. . 110.

Vertigan Müll.

Vertilla $\mathrm{M}, \mathrm{T}$.

Mehrinus Held . . . 33. 




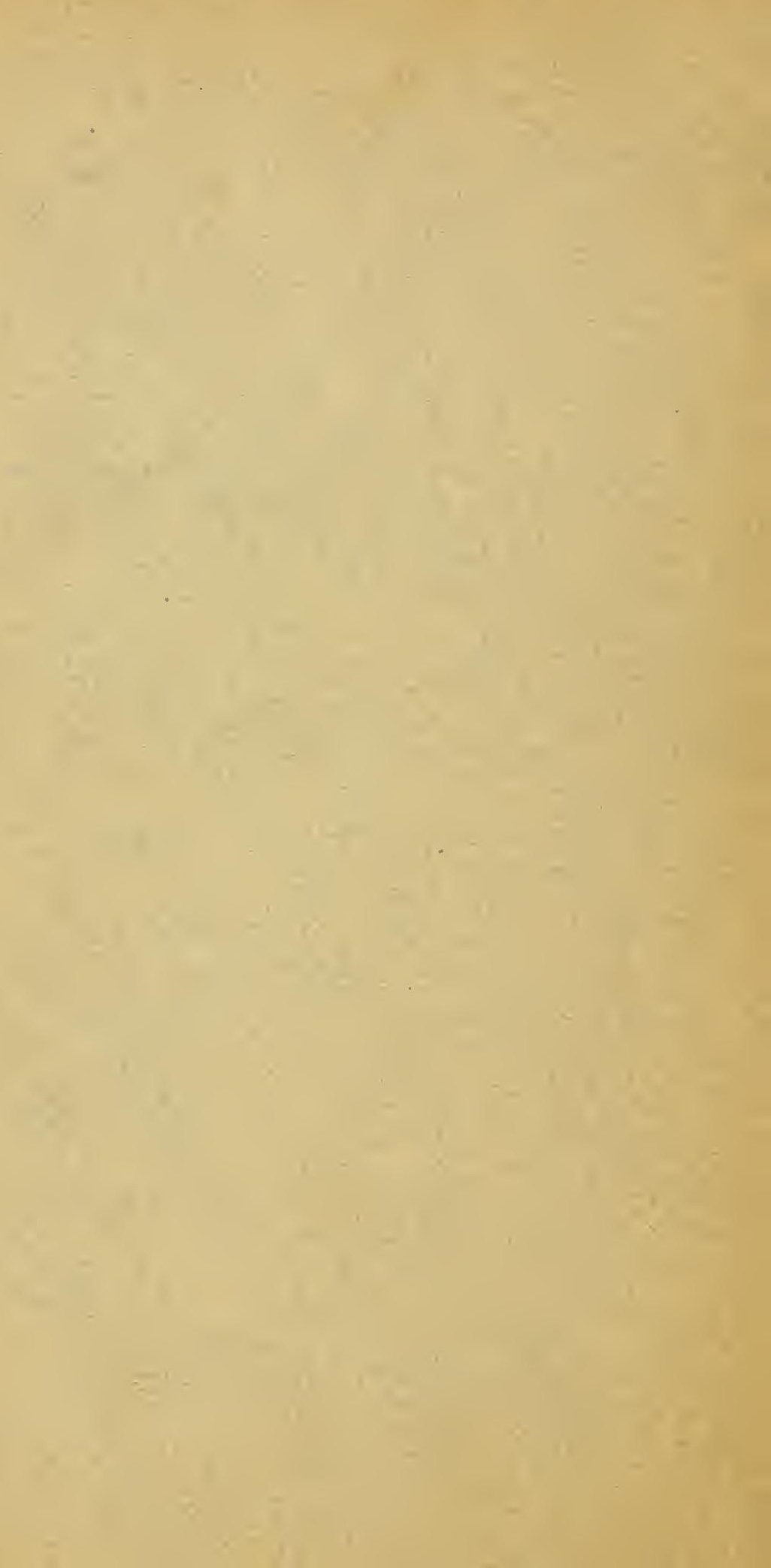




\section{PAMPHLET BINDERS}

This is No. 1524

aiso carried in tock in the fallowing sizes HIGH MDE THICKNESS

1523 inehes 7 inches $1 / 2$ juch

1524

1526

1527

$1528 \mathrm{i}$

a 78

7 is 1530

54 a 40

193416

H16H

-IDE THICKNESS

2 imebes 10 isches $1 / 3$ inch

is $\$ 1 / 8$ "

a 11 ये

* 12 "

Other slzes made to order.

MANUFACTURED BY

LIBRARY BUREAU

Division of REMINGTON RAND INC.

Library Supplies of all Kinde 


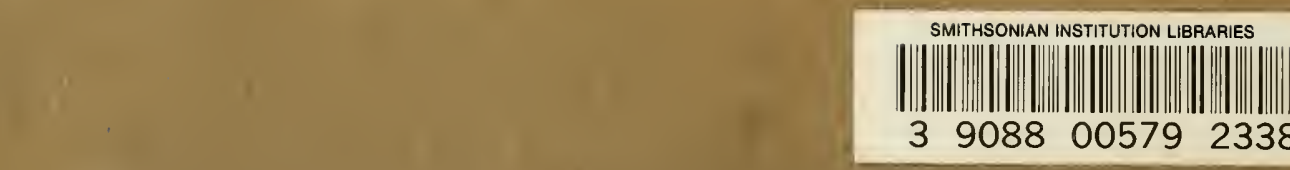

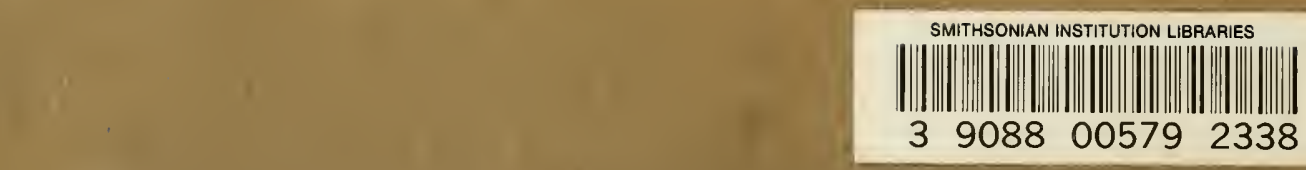
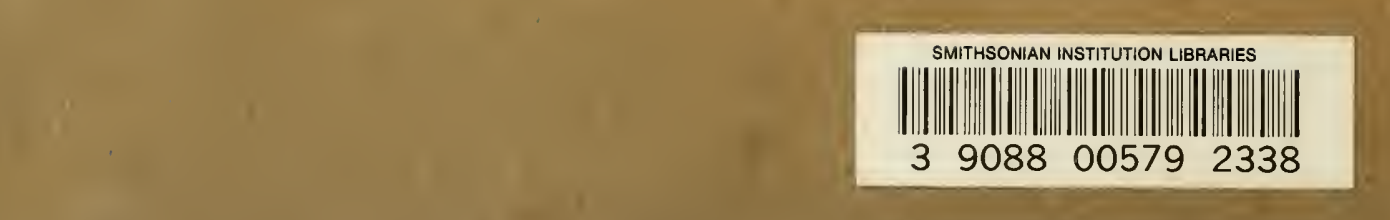

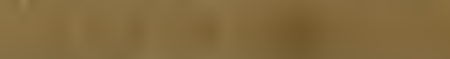

.

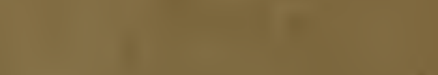

